\title{
Evaluierung \\ der HRSM-Ausschreibung 2013 zur Anschubfinanzierung von Kooperationen der Universitäten in Lehre und Forschung/Entwicklung und Erschließung der Künste sowie Verwaltung
}

\section{Evaluierung \\ der HRSM-Ausschreibung 2016 für Kooperationen im Bereich Lehre}

Brigitte Ecker, Clemens Danler, Gerald Gogola

(WPZ Research)

Anna Wang, Nikolas Reschen (AIT Austrian Institute of Technology) 


\section{Evaluierung \\ der HRSM-Ausschreibung 2013 zur Anschubfinanzierung von Kooperationen der Universitäten in Lehre und Forschung/Entwicklung und Erschließung der Künste sowie Verwaltung}

\section{Evaluierung \\ der HRSM-Ausschreibung 2016 für Kooperationen im Bereich Lehre}

Endbericht 


\section{Inhaltsverzeichnis}

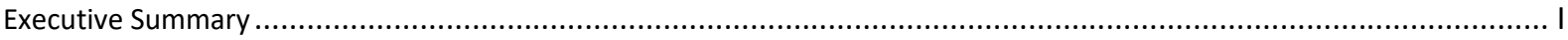

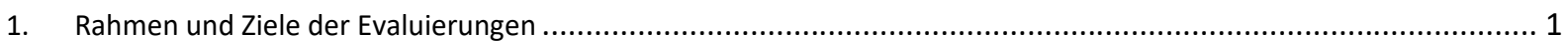

1.1 Die Hochschulraum-Strukturmittel als wichtige Säule der Universitätsfinanzierung in Österreich ....................... 1

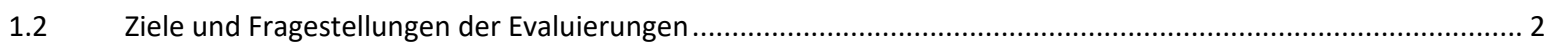

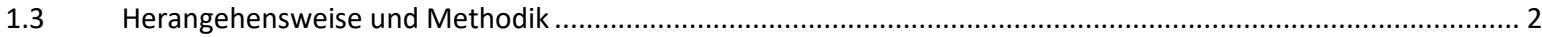

2. HRSM-Ausschreibung 2013 zur Anschubfinanzierung von Kooperationen der Universitäten in Lehre und Forschung/Entwicklung und Erschließung der Künste sowie Verwaltung ...................................................... 7

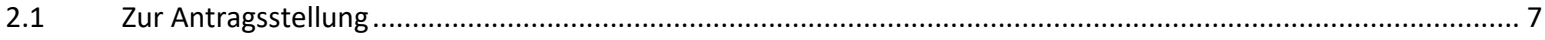

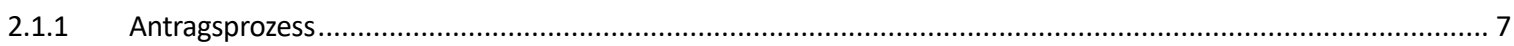

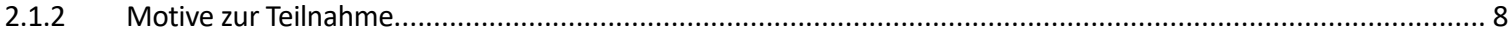

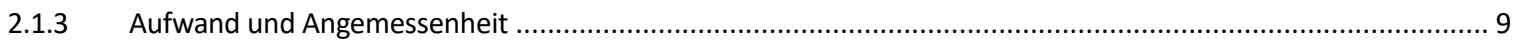

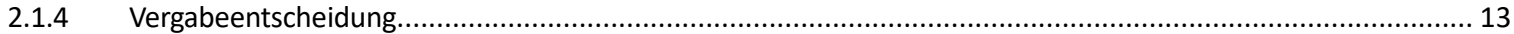

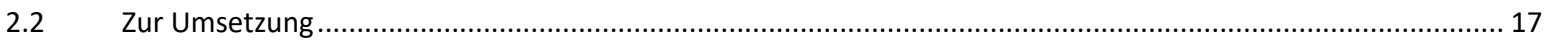

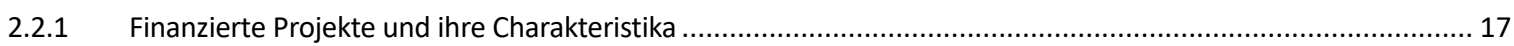

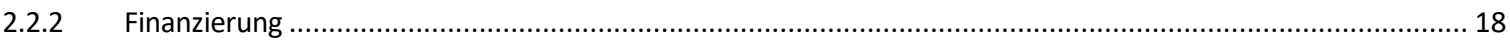

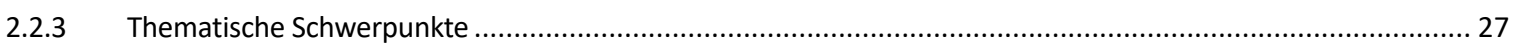

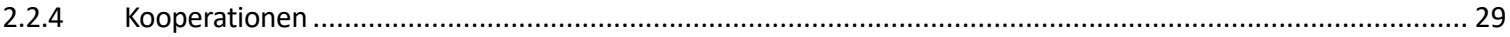

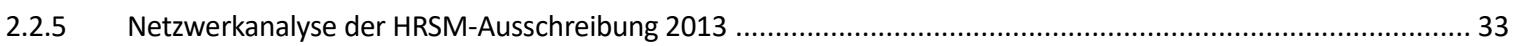

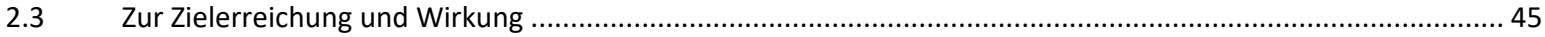

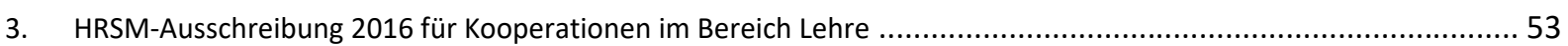

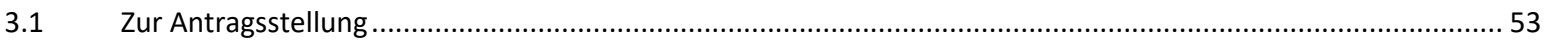

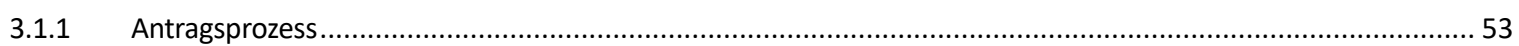

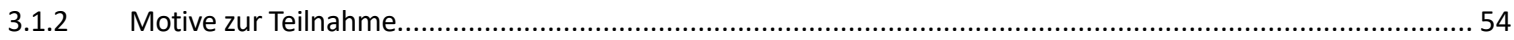

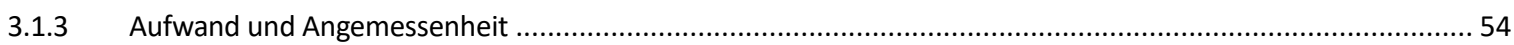

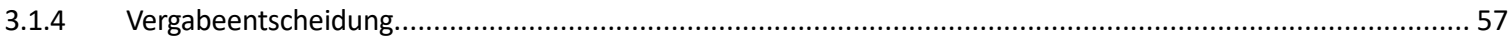

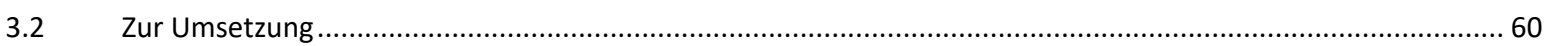

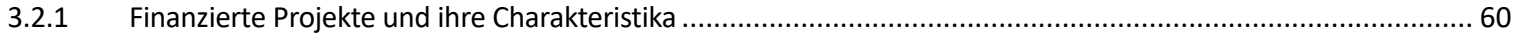

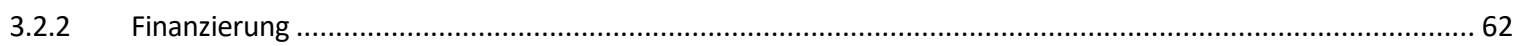

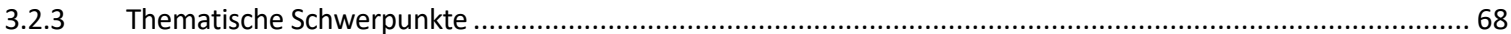

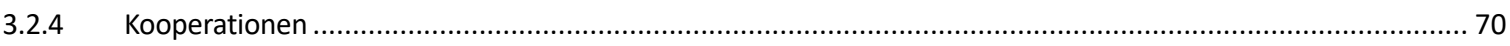

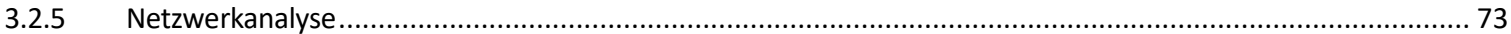

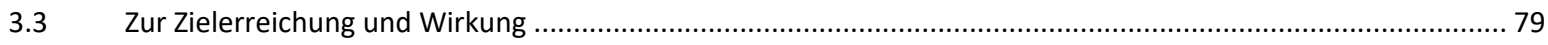

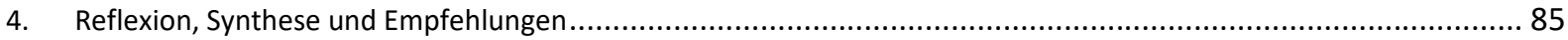

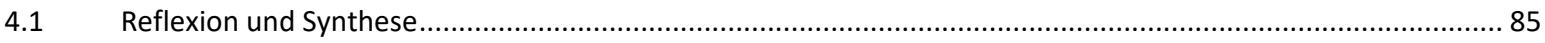

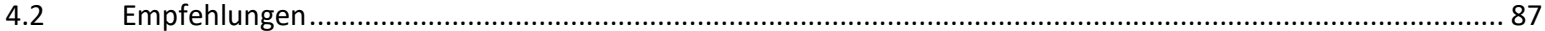

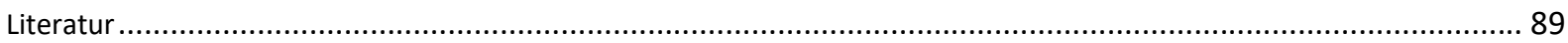

Anhang

Befragung der Projektleiterinnen und -leiter von finanzierten Projekten der HRSM Ausschreibung 2013.......................90

Befragung der Projektleiterinnen und -leiter von nicht-finanzierten Projekten der HRSM-Ausschreibung 2013 .............. 99

Befragung der Projektleiterinnen und -leiter von finanzierten Projekten der HRSM-Ausschreibung für

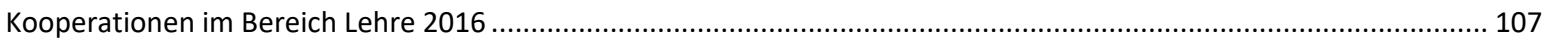

Befragung der Projektleiterinnen und -leiter von nicht-finanzierten Projekten der HRSM-Ausschreibung 2016 ............. 116 


\section{Executive Summary}

Die Hochschulraum-Strukturmittel (HRSM) waren bis 2018 Teil des Globalbudgets der Universitäten und erfüllten die Funktion eines leistungs- und outputorientierten Instruments in der öffentlichen Universitätsfinanzierung. In der Historie lösten die Hochschulraum-Strukturmittel das Formelbudget ab und waren diese auch gesetzlich verankert. In den Jahren 2013-2015 wurde das Instrument erstmalig für eine Ausschreibung eingesetzt, in der weiteren Folge wurden die HRSM dann dreimal in der Leistungsvereinbarungsperiode 2016-2018 verwendet.

Mit der vorliegenden Studie werden die HRSM-Ausschreibung 2013 zur Anschubfinanzierung von Kooperationen der Universitäten in Lehre und Forschung/Entwicklung und Erschließung der Künste sowie Verwaltung und die HRSM-Ausschreibung 2016 für Kooperationen im Bereich Lehre evaluiert. Im Fokus der Evaluierungen steht dabei vor allem die Frage nach der Wirkung des Instruments und damit einhergehend welche Potentiale in Zukunft bei weiteren Ausschreibungen gehoben werden können.

\section{Hochschulraumstrukturmittel 2013 zur Anschubfinanzierung von Kooperationen der Universitäten in Lehre und Forschung/Entwicklung und Erschließung der Künste sowie Verwaltung}

Im Rahmen der Hochschulraumstrukturmittel zur Anschubfinanzierung von Kooperationen der Universitäten in Lehre und Forschung/Entwicklung und Erschließung der Künste sowie Verwaltung hat das BMBWF 2013 eine Ausschreibung mit einem Vergabevolumen von insgesamt 63 Mio. Euro durchgeführt. Von den 218 beantragten Projekten wurden insgesamt 83 Projekte durch das BMBWF finanziert. Vom Finanzierungsvolumen entfielen $8 \%$ (5,17 Mio. Euro) auf den Teilbereich Lehre, 68 \% (42,83 Mio. Euro) auf den Teilbereich Forschung/EEK und $24 \%$ (15,0 Mio. Euro) auf den Teilbereich Verwaltung. Generell wurde maximal ein Drittel der gesamten Projektkosten durch das BMBWF finanziert.

Zwischen den Universitätstypen bestehen Unterschiede zwischen den Bewilligungsquoten, den beantragten und den tatsächlich vergebenen Mitteln. Die Volluniversitäten konnten die meisten Anträge stellen und mit durchschnittlich acht Projekten die meisten Bewilligungen einwerben. Die Kunstuniversitäten weisen dagegen mit durchschnittlich 1,8 finanzierten Projekten die wenigsten Projekte pro Universitätstyp auf. Volluniversitäten konnten mit rund $43 \%$ (26,9 Mio. Euro) die meisten Finanzierungsmittel einwerben, gefolgt von den Medizinischen Universitäten mit 21 \% (12,95 Mio. Euro), den Sonstigen Universitäten mit 17 \% (10,92 Mio. Euro), den Technischen Universitäten mit 15 \% (9,46 Mio. Euro) und den Kunstuniversitäten mit 4 \% (2,77 Mio. Euro).

Die Projekte, die durch die HRSM 2013 finanziert wurden, sind inhaltlich und thematisch breit gefächert. Knapp die Hälfte (47 \% bzw. 39 von 83 Projekte) hatten den Ausbau der Forschungsinfrastruktur zum Ziel. Inhaltlich erwarteten die Antragstellerinnen und Antragsteller im Bereich Lehre und Forschung/EEK vor allem eine nachhaltige Kooperation ( $85 \%$ ) und die Etablierung gemeinsamer Strukturen (75\%). Bei den Verwaltungsprojekten wurden insbesondere Effizienzsteigerungen durch konkrete Synergieeffekte erwartet. Die befragten Projektleiterinnen und -leiter sind in hohem Ausmaß davon überzeugt, dass die angestrebten Ziele der HRSM erreicht wurden. Die höchste Zielerreichung ist dabei bei der „Erhöhung der Sichtbarkeit“ (für 96 \% sehr bzw. eher gelungen), dem „Aufbau nachhaltiger Kooperationsstrukturen“ (für 85 \% sehr bzw. eher gelungen) und der „Stärkung der Exzellenz" (für 83 \% sehr bzw. eher gelungen) zu verzeichnen. Darüber hinaus haben die HRSM 2013 einen wesentlichen Beitrag zur Profilbildung der einzelnen Universitäten und zu einem abgestimmten Hochschul- und Forschungsraum in Österreich geleistet.

\section{Hochschulraumstrukturmittel 2016 für Kooperationen im Bereich Lehre}

Ein besonderer Schwerpunkt der HRSM-Ausschreibung 2016 im Bereich Lehre lag auf der Entwicklung und Umsetzung der PädagogInnenbildung Neu in vier Entwicklungsverbünden (West, Mitte, Nord-Ost und Süd-Ost). Durch diese Reform sollen Lehramtsstudien für allgemeinbildende Fächer an den Schulen der Sekundarstufe (Mittelschulen, Allgemeinbildende Höhere Schulen, Polytechnische Schulen, Mittlere und Höhere Berufsbildende Schulen) gemeinsam von den öffentlichen Universitäten und Pädagogischen Hochschulen eingerichtet und durchgeführt werden. Darüber hinaus wurden Sonstige Lehrprojekte finanziert, die als Impulsgeber für verstärkte Kooperationen in der Lehre dienen sollten. Insgesamt wurden 35,3 Mio. Euro vergeben, davon 
entfielen 84 \% (29,8 Mio. Euro) auf den Teilbereich PädagogInnenbildung Neu und 16 \% (5,5 Mio. Euro) auf den Teilbereich Sonstige Lehrprojekte. Von den 48 beantragten Projekten wurden insgesamt 32 Projekte durch das BMBWF finanziert.

Rund die Hälfte aller Projekte (47 \% bzw. 15 von 32 Projekten) wurde an den Volluniversitäten durchgeführt, gefolgt von den Sonstigen Universitäten mit sieben Projekten und den Kunstuniversitäten mit fünf Projekten. Dementsprechend entfiel mit 24,6 Mio. Euro (70\%) auch der größte Anteil der Finanzierung auf Volluniversitäten. Dahinter folgten die Sonstigen Universitäten (6,3 Mio. Euro), die Kunstuniversitäten (2,8 Mio. Euro), die Medizinischen Universitäten (0,85 Mio. Euro) und die Technischen Universitäten (0,78 Mio. Euro). Die inhaltlichen Schwerpunkte der Projekte im Bereich PädagogInnenbildung Neu sind erwartungsgemäß recht homogen. Der Fokus liegt auf dem Auf- und Ausbau von Personal und Infrastruktur für die gemeinsame Umsetzung der Lehramtsstudien im Verbund, inklusive der Schaffung von gemeinsamen Koordinationsstellen und der Optimierung/Harmonisierung von organisatorischen Prozessen. Die inhaltlichen Schwerpunkte der Projekte im Bereich Sonstige Lehrprojekte sind dagegen breit gefächert und reichen von gemeinsamen Aufnahme- und Auswahlverfahren, über gemeinsame IT-Infrastruktur bis hin zur Entwicklung von E-Learning Formaten.

Fast alle Projektleiterinnen und -leiter (92\%) sind im Rahmen der Online-Befragung der Meinung, dass ihr jeweiliges HRSM-Projekt die grundsätzliche Intention erfüllt hat. Am häufigsten wurde angegeben, dass der Aufbau neuer universitäts- und hochschulübergreifender Studienangebote (inkl. Lehramtsstudien) sehr gelungen ist, dahinter folgen die Stärkung der Verbundregionen zur Durchführung von Lehramtsstudien und der Einsatz von E-Learning Formaten. Abseits dieser Projektziele waren 85 \% der Meinung, dass die Projekte die nationale oder internationale Sichtbarkeit der Universität verbessert haben, und $81 \%$ waren der Ansicht, dass Synergieeffekte erzielt werden konnten. Des Weiteren haben die HRSM-Projekte einen Beitrag zur Profilbildung der jeweiligen Universität geleistet. Projekte der HRSM-Ausschreibung 2016 im Bereich PädagogInnenbildung Neu wurden als besonders nachhaltig empfunden, nicht zuletzt da diese systematisch in die Leistungsvereinbarungen 2019-2021 übernommen wurden.

Insgesamt ist das Bild der HRSM-Evaluierungen sehr positiv. Es wird belegt, dass die HochschulraumStrukturmittel eine wichtige Säule der Universitätsfinanzierung waren, insbesondere die über die HRSM finanzierten Personalressourcen werden in der aktuellen Leistungsvereinbarungsperiode weitergeführt und so haben auch einzelne HRSM-finanzierte Projekte - seit ihrer Anschubfinanzierung - bis heute deutlich an Sichtbarkeit gewonnen bzw. sind diese Projekte entsprechend institutionalisiert worden. Als erfolgreiches Beispiel kann hierfür das Climate Change Center Austria genannt werden, welches aus der HRSM-Ausschreibung 2013 hervorgegangen ist. Was die HRSM-Ausschreibung 2016 im Bereich Lehre betrifft, so galt es hier, ein politisches Ziel zu unterstützen, nämlich die PädagogInnenbildung Neu einzuführen. Schwierigkeiten bei der Umsetzung der HRSM-Projekte-2016, welche insbesondere die hochschulübergreifende Abstimmung zur gemeinsamen Umsetzung von Lehramtsstudien betroffen haben, sind vor allem dadurch zu begründen, dass unterschiedliche institutionelle Charakteristika, nämlich die autonom agierenden Universitäten auf der einen Seite und die Pädagogischen Hochschulen als nachgeordnete Dienststellen des Bundes auf der anderen Seite, aufeinandertrafen. Anders sind die Kooperationen im Rahmen der HRSM-Ausschreibung 2013 zu betrachten. Hier war die Herangehensweise eine andere. Im Rahmen der HRSM-Ausschreibung 2013 wurden vor allem universitätsübergreifende Kooperationen finanziert, die meist auf bereits bestehenden Kontakten oder auch Netzwerken beruhten. Geographische Nähe, gemeinsame thematische Schwerpunkte in Lehre und Forschung oder die Kostenreduktion in der Verwaltung waren ausschlaggebende Gründe für diese HRSM-finanzierten Kooperationen.

Was die Durchführung der Ausschreibungen betrifft, so wird diese als sehr gut bewertet. Nicht zuletzt war die durchführende Institution, das BMBWF, bestrebt, das Ausschreibungsverfahren sowie das Verfahren der Projektauswahl stets zu verbessern; d.h. beginnend mit der Setzung von Anreizen bzw. Impulsen in der HRSMAusschreibung 2013, die völlig themenoffen, bottom-up initiiert waren, ging man über, systemwirksame Veränderungen anzuzielen. Zu diesem Zweck wurde auch der Auswahlprozess professionalisiert, u.a. wurde externe Expertise hinzugezogen.

Tatsächlich besteht der Erfolgsfaktor von Kooperationen in deren Nachhaltigkeit. Die Netzwerkanalysen zeigen, dass die Kooperationsbündnisse (oder eben Cluster) sehr eigenständig agieren, es gibt kaum Überlappungen. 
Abgesehen von Einzelprojekten ist die Sichtbarkeit der durchgeführten Projekte im Sinne von Nachhaltigkeit bei vielen Projekten nicht gegeben. Angesichts dessen empfehlen wir für die nächsten Ausschreibungen, das Augenmerk verstärkt auf den Impact, d.h. auf die einzelnen Wirkungsdimensionen, von Projekten zu legen. Sowohl die Einbettung der Kooperationen im Kontext, in den einzelnen Strategien etc. wie auch die erwartbaren Ergebnisse, Wirkungen sollten bereits in der Antragstellung dargelegt und gegenüber dem BMBWF auch klar kommuniziert werden. Schließlich soll damit nicht nur die Verbindlichkeit, sondern auch das Bemühen gesteigert werden, sämtliche Potentiale auszuschöpfen, um eine größtmögliche Wirkung (auch unter dem Aspekt des effizienten Mitteleinsatzes) zu erzielen.

Die Hochschulraum-Strukturmittel waren für die Universitäten von hoher strategischer Relevanz. Projekte, die durch Hochschulraum-Strukturmittel finanziert werden, erlangten auch universitätsintern mehr Sichtbarkeit. Angesichts dessen gilt es auch, diese Relevanz nach außen zu tragen, die systemische Wirkung in Zukunft noch sichtbarer zu machen. 


\section{Rahmen und Ziele der Evaluierungen}

Die Hochschulraum-Strukturmittel (HRSM) waren bis 2018 Teil des Globalbudgets der Universitäten und erfüllten die Funktion eines leistungs- und outputorientierten Instruments in der öffentlichen Finanzierung der Universitäten. Für die Jahre 2013-2015 wurde das Instrument erstmalig für eine Ausschreibung eingesetzt und dann drei Mal in der Leistungsvereinbarungsperiode 2016-2018 verwendet. Mit der vorliegenden Studie werden die HRSM-Ausschreibung 2013 zur Anschubfinanzierung von Kooperationen der Universitäten in Lehre und Forschung/Entwicklung und Erschließung der Künste sowie Verwaltung und die HRSM-Ausschreibung 2016 für Kooperationen im Bereich Lehre evaluiert.

Im Fokus der Evaluierungen steht dabei vor allem die Frage nach der Wirkung des Instruments und damit einhergehend welche Potentiale in Zukunft noch bei weiteren Ausschreibungen gehoben werden können.

Bevor dieser zentralen Frage mittels vielfältiger Methoden nachgegangen wird, wird zuvor auf die Bedeutung der Hochschulraum-Strukturmittel in der Universitätsfinanzierung eingegangen. Vor diesem Hintergrund werden dann die Ziele und zentralen Fragestellungen der Evaluierungen dargelegt sowie die Herangehensweise und der Methodeneinsatz seitens des Evaluierungsteams erläutert.

\subsection{Die Hochschulraum-Strukturmittel als wichtige Säule der Universitätsfinanzierung in Österreich}

In der Historie haben die Hochschulraum-Strukturmittel das Formelbudget abgelöst und wurden diese erstmalig als Instrument in der Leitungsvereinbarungsperiode 2013-2015 eingesetzt. Als leistungs- und outputorientiertes Instrument waren die Hochschulraumstrukturmittel im § 12 UG verankert und durch eine Verordnung des damaligen Bundesministers für Wissenschaft, Forschung und Wirtschaft, akkordiert mit dem Bundesminister für Finanzen, konkretisiert (BMWFW, 2014).

Mit der Einführung der Hochschulraum-Strukturmittel wurde seitens der Hochschulpolitik prioritär das Ziel verfolgt, die Universitätsfinanzierung leistungsorientierter - auch hinsichtlich einer Steigerung der Mitteleffizienz und -effektivität zu gestalten - und damit einhergehend vor allem solche Projekte zu finanzieren, die von systemändernder Relevanz sind bzw. zumindest Potential für eine systemische Wirkung im Kreis der Antragstellerinnen und Antragsteller aufweisen. Nicht zuletzt wurde damals die Einführung der HochschulraumStrukturmittel auch bereits als Vorbereitung für eine neue Universitätsfinanzierung gesehen, die mittlerweile seit 1.1.2019 in Kraft ist.

Die Hochschulraum-Strukturmittel waren Teil des Globalbudgets der Universitäten, welcher als ergänzende Säule mittels kompetitiv eingeworbener Mittel zum Grundbudget dazu kam, das durch die Aufgaben der Universitäten und die Vorhaben und Aktivitäten, die jeweils in den Leistungsvereinbarungen festgehalten werden, determiniert war. Insgesamt standen den Universitäten für die Jahre 2013-2015 450 Mio. Euro an HochschulraumStrukturmitteln zur Verfügung. Diese Summe setzte sich wie folgt aus fünf Teilbeträgen zusammen: i) Teilbetrag für prüfungsaktiv betriebene ordentliche Studien (270 Mio. Euro), ii) Teilbetrag für Absolventinnen und Absolventen ordentlicher Studien (45 Mio. Euro), iii) Teilbetrag für eingeworbene Drittmittel - Wissenstransfer (63 Mio. Euro), iv) Teilbetrag für private Spenden (9 Mio. Euro) und v) Teilbetrag für Kooperationen (63 Mio. Euro). ${ }^{1}$ Letzterer Teilbetrag wurde für eine Anschubfinanzierung von interuniversitären Kooperationen und Kooperationen der Universitäten mit anderen Einrichtungen aus dem Bildungsbereich und/oder der Wirtschaft im Rahmen einer Ausschreibung den Universitäten zur Verfügung gestellt und ist Inhalt der ersten Evaluierung im vorliegenden Bericht.

Im Jahr 2016 folgten sodann weitere Hochschulraum-Strukturmittelausschreibungen (HRSM-Ausschreibungen) und zwar in den Bereichen Lehre (dotiert mit einer Summe von 35 Mio. Euro), Forschung (50 Mio. Euro) und Verwaltungsinnovationen (12,5 Mio. Euro). ${ }^{2}$ Bis dato wurden vor allem die Projekte der Ausschreibung "Lehre“ 
zur Gänze fertig umgesetzt. Die HRSM-Ausschreibung „Lehre“ hatte insbesondere das Ziel, die PädagogInnenbildung Neu zu unterstützen. Darüber hinaus wurden auch Einzelvorhaben mit den HRSM in der Lehre finanziert. Diese Ausschreibung ist Inhalt der zweiten Evaluierungsstudie des vorliegenden Berichts.

\subsection{Ziele und Fragestellungen der Evaluierungen}

Da nun alle Projekte der HRSM-Ausschreibung 2013 und der HRSM-Ausschreibung 2016 für den Teilbereich Lehre abgeschlossen sind, umfassen die Evaluierungen des vorliegenden Berichts die gesamte Projektlaufzeit beider Ausschreibungen. Die Intention der Evaluierungen ist es, die Erfahrungen aller beteiligten Akteurinnen und Akteure umfassend zu sammeln und darzustellen, wie auch Detailanalysen vorzunehmen, um letztlich die Wirkungen des HRSM-Instruments identifizieren zu können. Ein weiterer Aspekt besteht darin, Erfolgsfaktoren bzw. sofern möglich Erfolgsmuster für systemverändernde bzw. systemisch wirksame (im Sinne auch von Nachhaltigkeit) Kooperationsprojekte zu identifizieren.

Die Evaluierungen umfassen zu diesem Zweck die Betrachtung sowohl auf Programm- als auch auf Projektebene. Die inner- und interuniversitäre Projektgestionierung ist - wie vereinbart mit dem BMBWF - nicht Gegenstand der Evaluierungen.

Zielen die HRSM-Ausschreibungen 2013 und 2016 auf unterschiedliche Impulse bzw. Themen ab, so kann dennoch eine Reihe von Fragestellungen für beide Ausschreibungen und somit für die Evaluierungen in diesem Bericht als relevant angesehen werden:

- Hat die Ausschreibung als Impulsgeber für eine verstärkte Kooperation in Lehre und Forschung/ Entwicklung und Erschließung der Künste gedient?

- Welche Vorteile konnten aus den Kooperationen gewonnen werden?

- Können Unterschiede in den Kooperationen nach Typ und/oder Größe der Universitäten erkannt werden?

- Welche Schwerpunkte wurden in den Projekten verfolgt und inwieweit sind diese deckungsgleich mit den Programmzielen?

- Inwieweit haben die Hochschulraum-Strukturmittel zur Profilbildung der jeweiligen Universitäten beigetragen?

- Hatten bzw. haben die Hochschulraum-Strukturmittel eine Bedeutung für die interne Strategie und die Entwicklungsplanung der Universitäten?

- Waren die durch die Hochschulraum-Strukturmittel indizierten Kooperationen nachhaltig und haben die finanzierten Projekte eine langfristige strategische Verankerung erhalten?

- Hat sich die Gestaltung der Ausschreibung der Hochschulraum-Strukturmittel als erfolgreich erwiesen?

- Können Potenziale zur Verbesserung der inhaltlichen, organisatorischen und strukturellen Abwicklung identifiziert werden?

All diese Fragen werden unter Verwendung unterschiedlicher empirischer Methoden adressiert und aufgegriffen. Die Herangehensweise wie auch die Methodik wird im folgenden Abschnitt erläutert.

\subsection{Herangehensweise und Methodik}

Die Beantwortung der Fragestellungen bedarf einer strukturierten Herangehensweise und verschiedener Methoden. Diese sind in nachfolgender Abbildung schematisch dargestellt und werden in Folge im Überblick auch kurz beschrieben. 


\section{Abbildung 1: Schematische Darstellung der Evaluierungsmethoden}

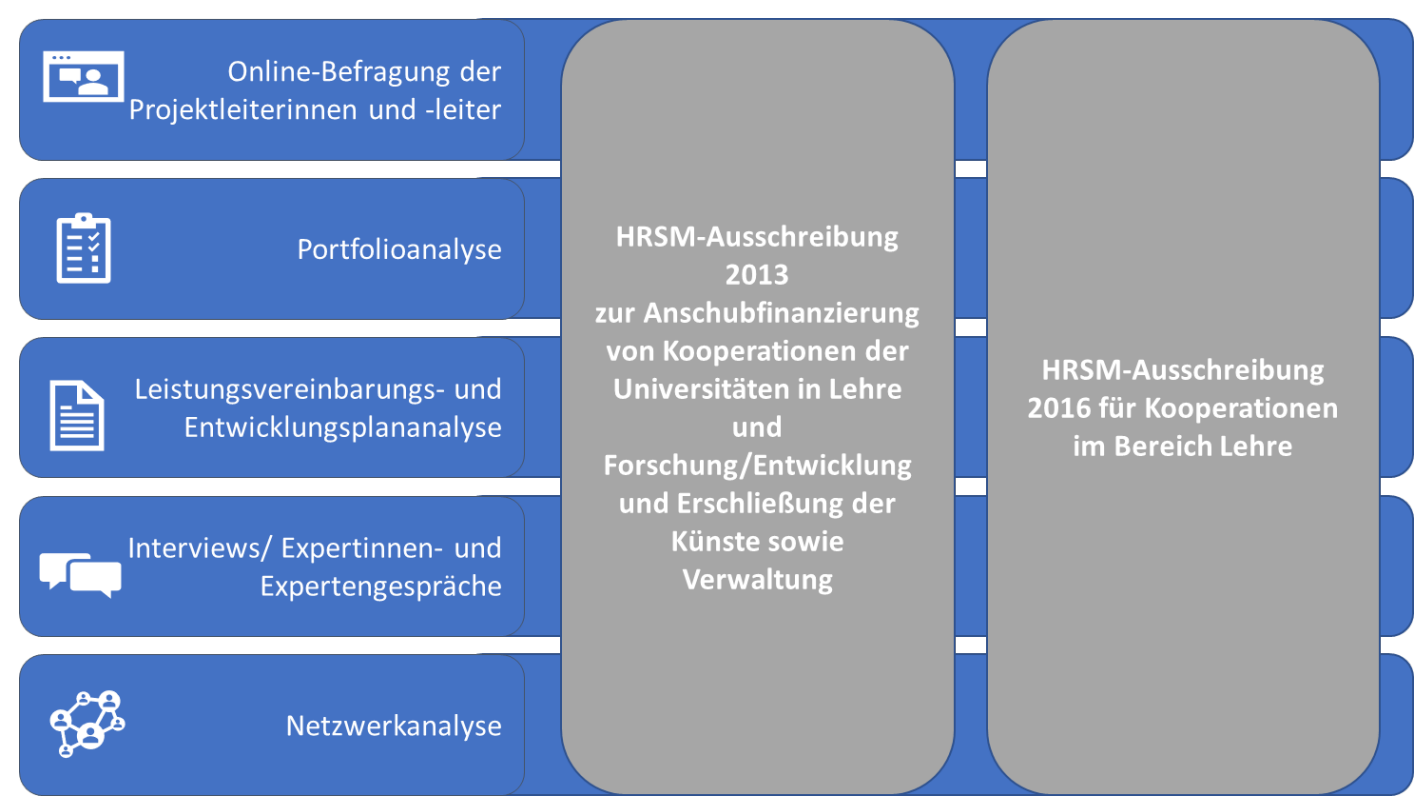

Quelle: eigene Darstellung

Online-Befragung: Ziel der Online-Umfrage ist es, zum einen die Projektleiterinnen und Projektleiter der finanzierten Projekte einzubeziehen und dabei Erkenntnisse zum Antragsverfahren, zum Umsetzungsprozess und zur nachhaltigen Wirkung auf Projektebene zu gewinnen, sowie zum anderen auch Antragstellerinnen und Antragsteller von nicht zur HRSM-Finanzierung ausgewählten Projekten zur Bedeutung und Durchführung der HRSM-Ausschreibungen zu befragen. Wo es inhaltlich und methodisch (Stichprobengröße) sinnvoll erscheint, werden die Antworten der Projektleiterinnen und -leiter getrennt nach Universitätstyp oder inhaltlichem Schwerpunkt des Projekts ausgewertet. Darüber hinaus werden in der Auswertung Filterfragen genutzt, die dazu dienen, den Kreis der Befragten anhand ihres Antwortverhaltens einzugrenzen.

Für die Evaluierungen wurden vier Online-Umfragen durchgeführt, die sich durch folgende Charakteristika (Beantwortungszeitraum, Rücklaufquote etc.) ausweisen:

\section{- Befragung der Projektleiterinnen und -leiter von finanzierten Projekten 2013}

Die Online-Befragung der Projektleiterinnen und -leiter von finanzierten Projekten wurde im Zeitraum von 19.06.2020 bis 20.07.2020 durchgeführt. Im Vorfeld wurde der Fragebogen unter Einbeziehung der Fachexpertise des BMBWF entwickelt und einem Test an zwei ausgewählten österreichischen Universitäten unterzogen. In einem nächsten Schritt informierte das BMBWF die Rektorate aller österreichischen Universitäten mittels eines Schreibens über die Durchführung der Evaluierung und bat um eine rege Teilnahme an der Umfrage. Nach Aussendung eines Reminders wurde der OnlineFragebogen von insgesamt 67 Projektleiterinnen und Projektleitern retourniert, sodass eine Rücklaufquote von $81 \%$ vorliegt. Es nahmen Projektleiterinnen und -leiter von 19 verschiedenen österreichischen Universitäten teil. Rund die Hälfte der befragten Personen haben für den Bereich Forschung Mittel der HRSM-Ausschreibung 2013 lukriert, ein Viertel im Bereich Lehre und ein Fünftel im Bereich Verwaltung. Aufgrund dieser Verteilung, die sich in gleichem Maße auch in der Auswertung der Projektanträge (siehe Kapitel 2.1) zeigt, kann geschlossen werden, dass die Ergebnisse der OnlineUmfrage repräsentativ für die Grundgesamtheit aller finanzierten Projekte sind.

\section{- Befragung der Projektleiterinnen und -leiter von nicht-finanzierten Projekten 2013}

Neben den Projektleiterinnen und -leitern von finanzierten Projekten wurden auch jene Personen befragt, die einen Antrag für ein HRSM-Projekt 2013 eingereicht hatten, ihr Projekt allerdings nicht vom BMBWF bewilligt und folglich auch nicht finanziert wurde. Ziel der Miteinbeziehung der nichtfinanzierten Projekte in der Evaluierung der HRSM-Ausschreibung 2013 ist es, einerseits dadurch die Validität der Ergebnisse zu erhöhen und andererseits auch mögliche Selektionseffekte zu verhindern. Die potentiellen, jedoch nicht-finanzierten Projektleiterinnen und -leiter hatten - ebenso wie die 
finanzierten Projektleiterinnen und -leiter - im Zeitraum von 19.06.2020 bis 20.07.2020 die Gelegenheit, an der Online-Befragung teilzunehmen. Zuvor waren die Rektorate mittels eines Schreibens seitens des BMBWF über die Evaluierung der HRSM-Ausschreibung sowie deren Vorgehensweise informiert worden.

Nach Aussenden eines Reminders nahmen schließlich insgesamt 34 Personen an der Befragung teil, dies entspricht einer Rücklaufquote von $25 \%$. Darüber hinaus haben noch 20 Adressierte zurückgemeldet, warum es ihnen nicht möglich ist, an der Befragung teilzunehmen. Zu den Gründen zählten häufig: Wechsel des Arbeitsplatzes an eine andere Institution im Inland wie auch ins Ausland, Pensionierung und/oder das Fehlen von vorliegenden Informationen den Antrag betreffend. Einzelne Adressierte haben auch angemerkt, dass der Zeitabstand zwischen Befragung und Projektantragstellung zu lange ist und es daher schwierig ist, die Fragen der Online-Umfrage heute richtig zu beantworten.

Für den Bericht gilt: Sofern sich die Befragungsergebnisse maßgeblich von jenen der finanzierten Projekte unterscheiden, werden diese an der entsprechenden Stelle in einer grauen Box - symbolisiert durch eine Weggabelung - dargestellt

\section{- Befragung der Projektleiterinnen und -leiter von finanzierten Projekten 2016}

Ebenso wie für die Analyse der HRSM 2013 wurden alle Projektleiterinnen und -leiter von finanzierten Projekten der HRSM-Ausschreibung 2016 eingeladen, an einer Online-Befragung teilzunehmen. Zuvor wurde der Fragebogen von zwei ausgewählten Universitäten getestet, sowie die Rektorate durch das BMBWF kontaktiert und um ihre Mithilfe gebeten. Die Online-Umfrage wurde sodann im Zeitraum von 22.06.2020 bis 20.07.2020 durchgeführt. Nach Aussendung eines Reminders wurde der Fragebogen von 27 Personen retourniert, sodass eine Rücklaufquote von $84 \%$ vorliegt. Vertreterinnen und Vertreter von 11 Universitäten beteiligten sich an der Umfrage. Mit $60 \%$ entfallen etwas mehr als die Hälfte der Projekte auf Maßnahmen im Bereich der PädagogInnenbildung Neu, $40 \%$ auf sonstige Lehrprojekte. Das entspricht nahezu dem Verhältnis, das bereits im Rahmen der Analyse der Projektanträge festgehalten wurde. Auch wenn damit ein repräsentativer Querschnitt für alle finanzierten Projekte der HRSM 2016 vorliegt, sind die nachfolgenden Auswertungen mit Vorsicht zu interpretieren, da mit 27 Beantwortungen nur sehr geringe Fallzahlen vorliegen. Bereits wenige Antworten haben prozentuell gesehen ein relativ hohes Gewicht.

\section{- Befragung der Projektleiterinnen und -leiter von nicht-finanzierten Projekten 2016}

Zeitgleich mit der Befragung der Projektleiterinnen und -leiter von finanzierten Projekten wurde zwischen 22.06.2020 bis 20.07.2020 auch eine Online-Befragung der Projektleiterinnen und -leiter von nicht-finanzierten Projekten durchgeführt. Auch hier wurden die Rektorate seitens des BMBWF vorab über die Evaluierung informiert. Selbst nach Aussendung eines Reminders retournierten lediglich sechs Personen den Fragebogen. Da auch die Grundgesamtheit aller nicht-finanzierten Projekte lediglich 16 beträgt, liegt die Rücklaufquote dennoch bei $38 \%$. Einzelne Leiterinnen und Leiter von nichtfinanzierten Projekten brachen den Online-Fragebogen allerdings vorzeitig ab, sodass zum Teil nur 2-3 Beantwortungen vorliegen. Daraus lassen sich keine validen Ergebnisse ermitteln, sodass an dieser Stelle keine Auswertung und Interpretation der Fragebögen durchgeführt werden konnten und hier auf die Befunde der qualitativen Interviews verwiesen wird.

Zusätzlich zu den Online-Befragungen wurden noch sämtliche Daten und Informationen aus den Projektanträgen und -berichten wie auch die Leistungsvereinbarungen und Entwicklungspläne für die Evaluierungen herangezogen. Die Interviews und Expertinnen- und Expertengespräche dienten insbesondere dazu, Erkenntnisse aus vorangehenden Analysen zu reflektieren bzw. noch nähere Auskünfte zu erzielen. Nicht zuletzt steht das Kooperationsverhalten immer wieder im Fokus der einzelnen Analysen. Visuell wird dieses auch durch die Netzwerkanalyse abgebildet. In Folge wird noch auf diese Methoden separat kurz eingegangen.

Portfolioanalyse: Die Portfolioanalyse dient der systematischen Darstellung der HRSM-finanzierten Projekte im Rahmen der Ausschreibungen 2013 und 2016. Die Portfolioanalyse hat auch das Ziel, Muster zu erkennen bzw. Erfolgsfaktoren zu identifizieren. Hierfür näher untersucht werden Kriterien wie etwa Finanzierungsvolumen, Projekttyp, Institution, Themenschwerpunkt, Zielgruppe, Nachhaltigkeit und das Erfüllen von inhaltlichen Vergabekriterien. 
Leistungsvereinbarungs- und Entwicklungsplananalyse: Durch die nähere Betrachtung der Leistungsvereinbarungen und der Entwicklungspläne der Universitäten sowie die damit einhergehende „in Kontext-Setzung“ mit den HRSM-finanzierten Projekten kann aufgezeigt werden, inwieweit die Universitäten eine strategische Verankerung der HRSM-finanzierten Projekte ernst genommen haben und die HRSM-Projekte damit eine nachhaltige Wirkung erzielten bzw. erzielen.

Interviews/Expertinnen- und Expertengespräche: Interviews und Expertinnen- und Expertengespräche dienen dazu, einen vertiefenden wie auch ergänzenden Einblick zu gewinnen sowie Erfahrungen, Ansichten und Anregungen auf qualitativem, narrativem Weg abzuholen. Insgesamt wurden 17 Personen auf diesem Weg im September und Oktober 2020 in die Evaluierung einbezogen.

Netzwerkanalyse: Ziel der Netzwerkanalyse ist es, die Kooperationsstrukturen - wer mit wem und in welcher Intensität in welchem Bereich vor welchem Hintergrund - darzulegen und daraus weitere Hinweise für eine bessere Abstimmung des Hochschulraums zu gewinnen. Die Netzwerke werden mit Hilfe des statistischen Programms R sowie des Erweiterungspakets ggplot2 erstellt. Die Matrix der Projekte wird dabei zu einer paarweisen Liste mit bilateralen Partnerschaften umgeformt, mit der in weiterer Folge die Netzwerke gebildet werden. Dabei werden die Netzwerke auf Basis des „Backbone graph layout“ kalkuliert, welches verborgene Strukturen in Netzwerken illustriert. Zentrale Indikatoren, die für die Analyse relevant sind, beinhalten:

- Die Anzahl der Partnerschaften als Verbindungen („Edges“);

- Eigenvector Centrality (EC), welche den Einfluss einer Organisation („Node“) auf einer Skala von 0 bis 1 misst; Nodes mit hohen EC-Werten weisen dabei viele Verbindungen mit anderen, tendenziell ebenfalls einflussreichen, Nodes auf;

- Die Position der Nodes innerhalb des Netzwerkes, wobei eine Position in der Mitte auf eine zentrale Rolle innerhalb des Netzwerkes hindeutet, während eine Position in der Peripherie auf einen weniger starken Einfluss verweist.

Info-Box:

Eigenvector Centrality oder Eigenvektorzentralität ${ }^{3}$

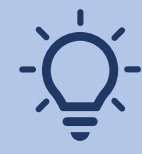

Im Gegensatz zu anderen Zentralitätsmaßen basiert der Eigenvektoransatz nicht auf Basis des unmittelbaren Umfeldes eines Knotens („Nodes“) (wie etwa die Degree-Zentralität), sondern kalkuliert die gesamte Netzwerkstruktur in den Berechnungsprozess ein. Diese Methode basiert darauf, dass ein Knoten wichtiger ist, wenn er wichtige Nachbarn hat. Der Zentralitätswert eines Knotens wird deshalb basierend auf der Summe der Werte der Nachbarn errechnet. Trotz der dadurch entstehenden Rückkopplungen lassen sich aussagekräftige Werte berechnen. Zur Erreichung einer Eindeutigkeit, wird der EC als Eigenvektor zum größten Eigenwert definiert. Berechnen lassen sich die EC-Werte eines Netzwerkes durch eine Faktorenanalyse, durch die Konvergenz einer infiniten Sequenz oder durch die Lösung eines linearen Gleichungssystems.

Um die Unterschiede und Gemeinsamkeiten der Projekte im Zuge der HRSM-Ausschreibungen 2013 und 2016 zu untersuchen, werden sie zum Teil nach Universitätstyp geclustert. In Abstimmung mit dem Universitätscontrolling des BMBWF wurde folgende Kategorisierung festgelegt:

\section{Volluniversitäten:}

- Universität Wien

- Universität Graz

- Universität Innsbruck

- Universität Salzburg

\section{Technische Universitäten:}

- Technische Universität Wien

- Technische Universität Graz

- Montanuniversität Leoben

3 Vgl. Leifeld und Malang (2009). 
HRSM-Evaluierung 2013 und 2016 - 6

\section{Medizinische Universitäten:}

- Medizinische Universität Wien

- Medizinische Universität Graz

- Medizinische Universität Innsbruck

\section{Sonstige Universitäten:}

- Universität für Bodenkultur Wien

- Veterinärmedizinische Universität Wien

- Universität Linz

- Universität Klagenfurt

- Wirtschaftsuniversität Wien

\section{Kunstuniversitäten:}

- Universität für angewandte Kunst Wien

- Universität für Musik und darstellende Kunst Wien

- Universität Mozarteum Salzburg

- Universität für Musik und darstellende Kunst Graz

- Universität für künstlerische und industrielle Gestaltung Linz

- Akademie der bildenden Künste Wien 


\section{HRSM-Ausschreibung 2013 zur Anschubfinanzierung von Kooperationen der Universitäten in Lehre und Forschung/Entwicklung und Erschließung der Künste sowie Verwaltung}

Für die HRSM-Ausschreibung 2013 standen insgesamt 63 Mio. Euro, welche im Rahmen eines kompetitiven Verfahrens vom BMBWF vergeben wurden, als Anschubfinanzierung für verschiedene strategische Kooperationsprojekte an Österreichs Universitäten zur Verfügung. Von dieser Gesamtsumme wurden 5,17 Mio. Euro (8 \%) dem Bereich Lehre, 42,83 Mio. Euro (68\%) dem Bereich Forschung/Entwicklung und Erschließung der Künste, sowie 15,0 Mio. Euro (24\%) dem Bereich Verwaltung zugesprochen. Voraussetzung für eine Teilnahme am Ausschreibungsverfahren war die Beteiligung zumindest einer weiteren Institution aus dem Wissenschafts-, Hochschul-, Kunst- oder Kulturbereich oder der Wirtschaft an dem Projekt (BMWFW, 2014). Der Start der finanzierten Projekte fiel in die Leistungsvereinbarungsperiode 2013 - 2015. Im Durchschnitt liefen die Projekte rund fünf Jahre, d.h. von 2013 bis 2018.

Im Folgenden wird die HRSM-Ausschreibung näher dargestellt und nachvollzogen. Dabei werden in einem ersten Schritt die Antragstellung, dann die Umsetzung der Projekte und abschließend deren Zielerreichung und Wirkung untersucht. Die Evaluierung wird mittels Methodentriangulation durchgeführt, d.h. es werden unterschiedliche qualitative und quantitative Methoden miteinander kombiniert, konkret sind dies quantitative Auswertungen der Projektanträge, Online-Befragungen von Leiterinnen und -leitern von finanzierten und nicht-finanzierten Projekten, eine Portfolioanalyse sowie eine Netzwerkanalyse. Die Ergebnisse dieser Analyseschritte werden nach Antragstellung, Umsetzung sowie Zielerreichung und Wirkung verschränkt dargestellt. Die Ergebnisse und Analysen der nicht durch HRSM finanzierten Projekte sind dabei stets mit dem Symbol einer Weggabelung versehen. Des Weiteren symbolisiert die Weggabelung, dass sich die Ansichten und Einschätzungen zwischen HRSM-finanzierten und nicht durch HRSM finanzierten Projekten zum Teil deutlich unterscheiden.

\subsection{Zur Antragsstellung}

Die erste Analyse geht auf den Prozess der Antragstellung ein und stellt damit den Antragsprozess, die Motive zur Teilnahme, den Aufwand für die Antragstellung sowie die Qualität der Vergabeentscheidung ins Zentrum der Betrachtung.

\subsubsection{Antragsprozess}

Insgesamt wurden 218 Projekte im Rahmen der HRSM-Ausschreibung 2013 eingereicht, 83 (38 \%) davon wurden schließlich genehmigt und erhielten eine Finanzierung seitens des BMBWF. Die Projektauswahl erfolgte in einem wettbewerblichen Verfahren. Mit Ausnahme der Donau-Universität Krems waren alle öffentlichen Universitäten in Österreich berechtigt, Anträge im Rahmen der HRSM-Ausschreibung 2013 zu stellen. Konnte die DonauUniversität Krems aufgrund ihrer besonderen Finanzierungssituation zwar nicht Antragstellerin sein, so war sie jedoch berechtigt, sich als Kooperationspartnerin an der HRSM-Ausschreibung 2013 zu beteiligen. Bis auf die Universität für Musik und darstellende Kunst Wien haben alle verbleibenden 20 öffentlichen Universitäten bei der HRSM-Ausschreibung 2013 Projektanträge gestellt.

Aus der Befragung der Projektleiterinnen und -leiter von finanzierten Projekten geht hervor, dass mit $90 \%$ in der überwiegenden Mehrheit der finanzierten HRSM-Projekte die Initiative zur Antragsstellung von der jeweiligen projektleitenden Universität ausging. Nur in $10 \%$ der Fälle motivierte eine Kooperationspartnerin bzw. ein Kooperationspartner die jeweilige Universität zur Antragsstellung.

Bei fast der Hälfte der Projekte (46\%) bereiteten die Projektleiterinnen und -leiter die Anträge an das BMBWF 1-3 Monate vor der Einreichung vor. 31 \% taten dies mit 3-6 Monaten über einen längeren Zeitraum hinweg (siehe Abbildung 2). 
Abbildung 2: Vorbereitung der Antragstellung

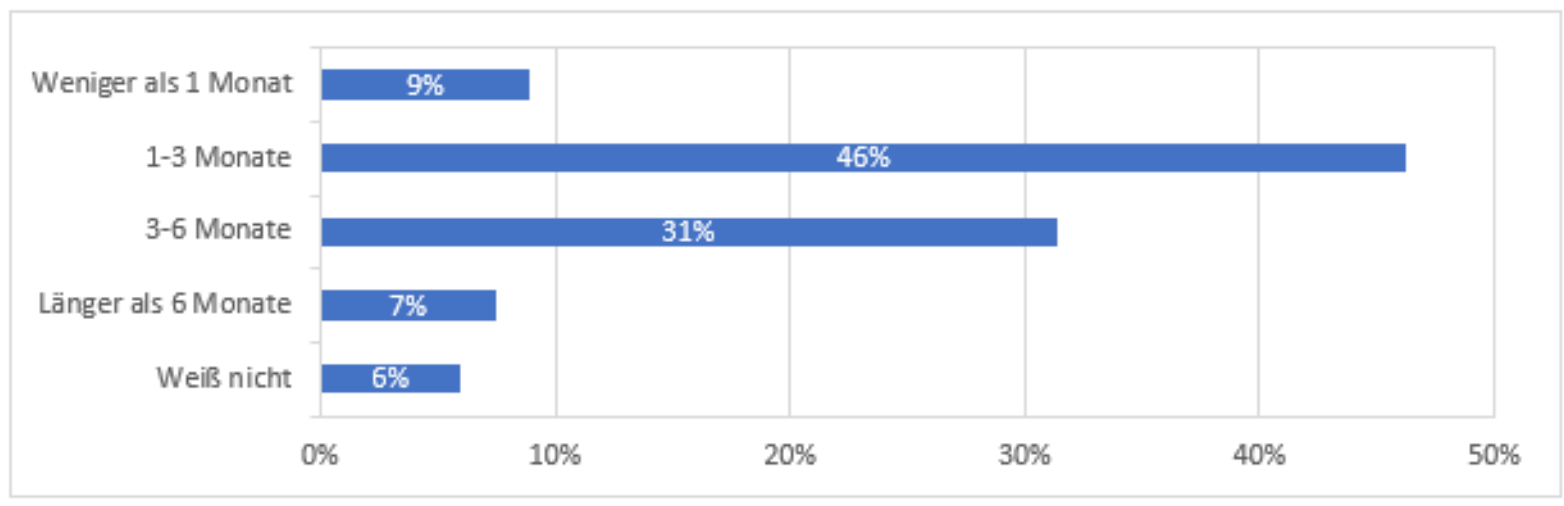

Quelle: WPZ Research Befragung der Projektleiterinnen und -leiter von finanzierten Projekten der HRSM-Ausschreibung 2013, $n=67$

Die Vorbereitung der Antragstellung erfolgte bei $56 \%$ der nicht-finanzierten Projekte 1-3 Monate vor der Einreichung, bei $21 \%$ 3-6 Monate davor und bei $12 \%$ weniger als ein Monat davor. Im Schnitt lag die Vorbereitungsdauer damit unter jener der finanzierten Projekte.

\subsubsection{Motive zur Teilnahme}

Wie in Abbildung 3 dargestellt, war der häufigste Grund für die Teilnahme an der HRSM-Ausschreibung 2013 der Aufbau nachhaltiger Kooperationsstrukturen. Mit $63 \%$ führten über die Hälfte der Projektleiterinnen und Projektleiter dieses Motiv an. Dahinter folgen mit $57 \%$ die Stärkung der Exzellenz und mit $48 \%$ der Auf- und Ausbau von Forschungsinfrastruktur. Projekte im Bereich der Lehre spielen im Rahmen der HRSM-Ausschreibung 2013 eine eher untergeordnete Rolle, dementsprechend gaben auch nur $18 \%$ an, die HRSM-Ausschreibung zum Aufbau neuer gemeinsamer Lehrformate/Studienangebote nutzen zu wollen. Ebenso entfielen lediglich $16 \%$ auf die Weiterentwicklung des bestehenden Lehrangebots.

Werden die Gründe für eine Teilnahme nach dem inhaltlichen Schwerpunkt des jeweiligen Projekts untergliedert, so zeigen sich z.T. große Unterschiede zwischen Lehre, Forschung, EEK und Verwaltung. Jene HRSM-Projekte, die einen Forschungsschwerpunkt aufweisen, gaben insbesondere die Stärkung der Exzellenz (81 \%) und den Aufund Ausbau von Forschungsinfrastruktur (77\%) als Hauptmotive für die Teilnahme an. Lehrprojekte zielten speziell auf den Aufbau nachhaltiger Kooperationsstrukturen (81\%) und den Aufbau neuer gemeinsamer Lehrformate/Studienangebote (56\%) ab. Bei HRSM-Projekten mit Schwerpunkt auf Entwicklung und Erschließung der Künste war mit 100 \% der Aufbau nachhaltiger Kooperationsstrukturen der Hauptgrund für die Teilnahme an der Ausschreibung. Wenig überraschend stand bei Verwaltungsprojekten die Verbesserung von Verwaltungsstrukturen/-prozessen im Mittelpunkt, 79 \% gaben dieses Motiv für die Teilnahme an. 


\section{Abbildung 3: Gründe für die Teilnahme an der HRSM-Ausschreibung}

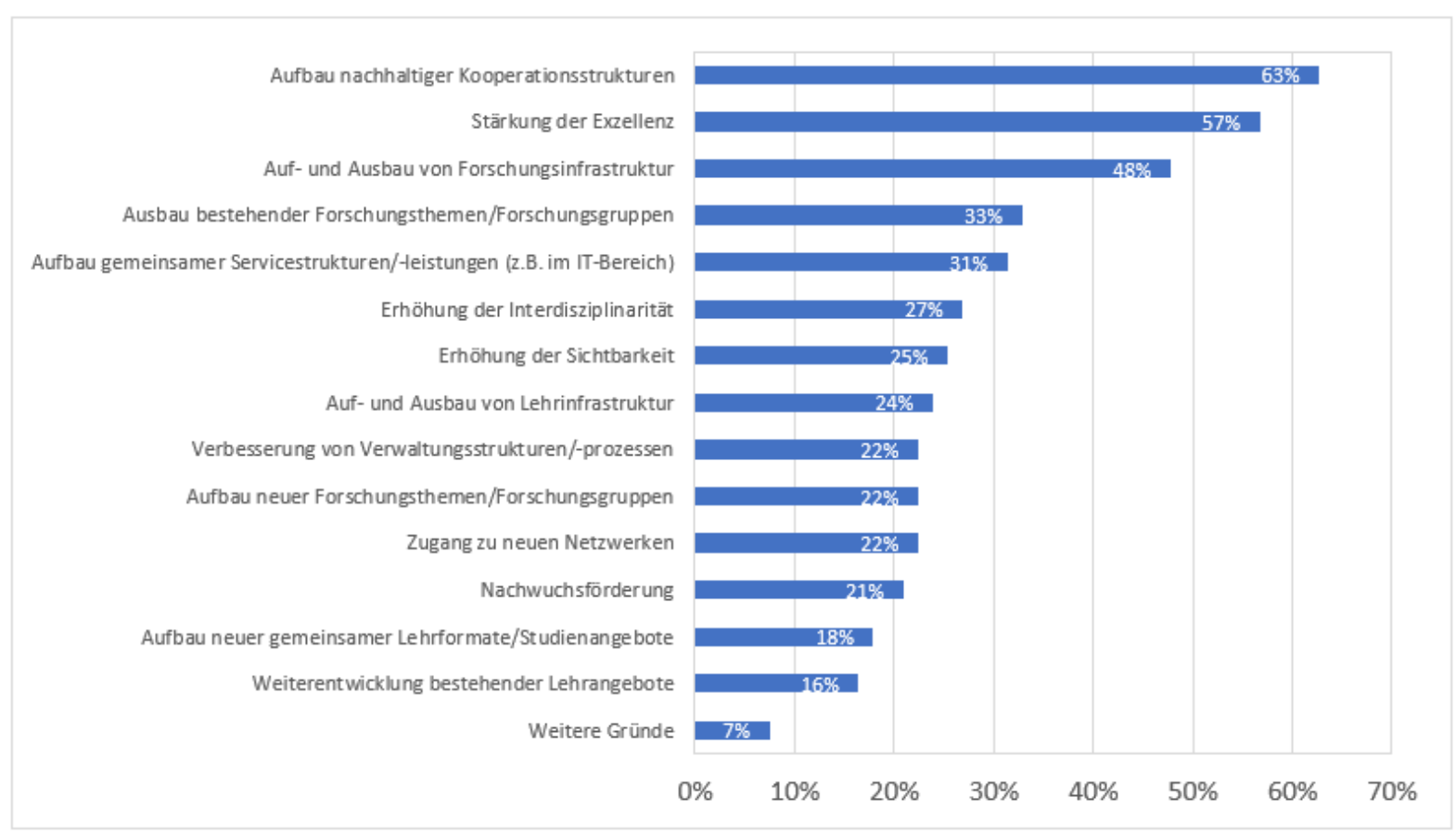

Quelle: WPZ Research Befragung der Projektleiterinnen und -leiter von finanzierten Projekten der HRSM-Ausschreibung 2013, $\mathrm{n}=67$

Die Gründe für die Teilnahme an der HRSM-Ausschreibung 2013 unterscheiden sich bei den nichtfinanzierten Projekten nur leicht von jenen der finanzierten Projekte. Wiederum waren der Auf- und Ausbau von Forschungsinfrastruktur, die Stärkung der Exzellenz und der Aufbau nachhaltiger Kooperationsstrukturen die Top-Motive. Der Aufbau nachhaltiger Kooperationsstrukturen lag bei den finanzierten Projekten allerdings an der Spitze und hat hier mit $63 \%$ um zehn Prozentpunkte mehr Zustimmung zu verzeichnen. Der Aufbau gemeinsamer Servicestrukturen/-leistungen (z.B. im IT-Bereich) lag bei den finanzierten Projekten mit $31 \%$ Zustimmung im vorderen Bereich, bei den nicht-finanzierten Projekten liegt dieses Motiv dagegen am vorletzten Rang (9\%).

\subsubsection{Aufwand und Angemessenheit}

Die Ausschreibungsunterlagen der HRSM 2013 empfanden die Projektleiterinnen und -leiter zu $95 \%$ als (eher) nachvollziehbar in Bezug auf Ziele und Anforderungen (siehe Abbildung 4). Lediglich $3 \%$ der befragten Personen gaben an, dass die Ausschreibungsunterlagen (eher) nicht nachvollziehbar waren. Dies ist daher als sehr positiv zu beurteilen. 
Abbildung 4: Nachvollziehbarkeit der Ausschreibungsunterlagen in Bezug auf Ziele und Anforderungen

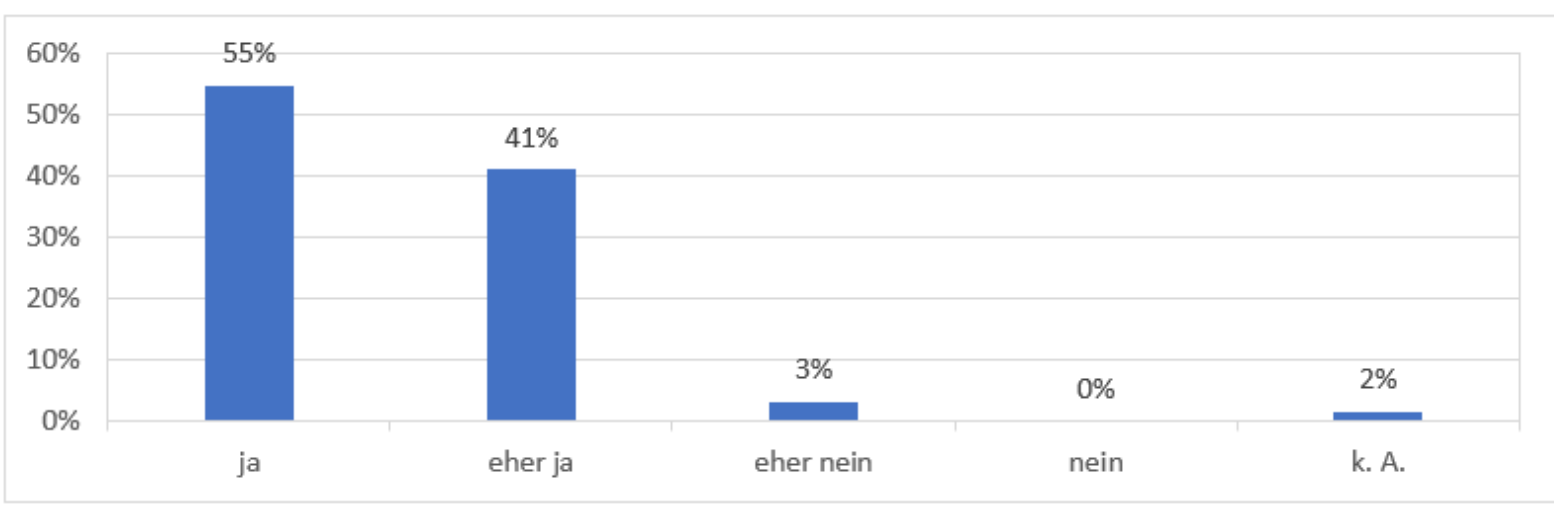

Quelle: WPZ Research Befragung der Projektleiterinnen und -leiter von finanzierten Projekten der HRSM-Ausschreibung 2013, $\mathrm{n}=66$

Den administrativen Aufwand zur Antragsstellung der HRSM 2013 schätzten 59 \% und damit etwas mehr als die Hälfte der Projektleiterinnen und -leiter als mittelmäßig ein. $17 \%$ empfanden ihn als hoch, etwa gleich viele als niedrig. Es dürfte dem BMBWF daher gelungen sein, die Antragsstellung so zu gestalten, dass sie inhaltlich nachvollziehbar und nicht zu aufwändig war. Je nach inhaltlichem Schwerpunkt des Projekts nahmen die Projektleiterinnen und Projektleiter den administrativen Aufwand allerdings sehr unterschiedlich wahr. Bei Lehrprojekten wird ein höherer Aufwand wahrgenommen als bei Forschungsprojekten. Wenn die Projekte inhaltliche Schwerpunkte im Bereich Forschung oder im Bereich Verwaltung verfolgten, so wurde überdurchschnittlich oft angegeben, dass der Aufwand mittel war.

\section{Abbildung 5: Administrativer Aufwand für die Antragsstellung}

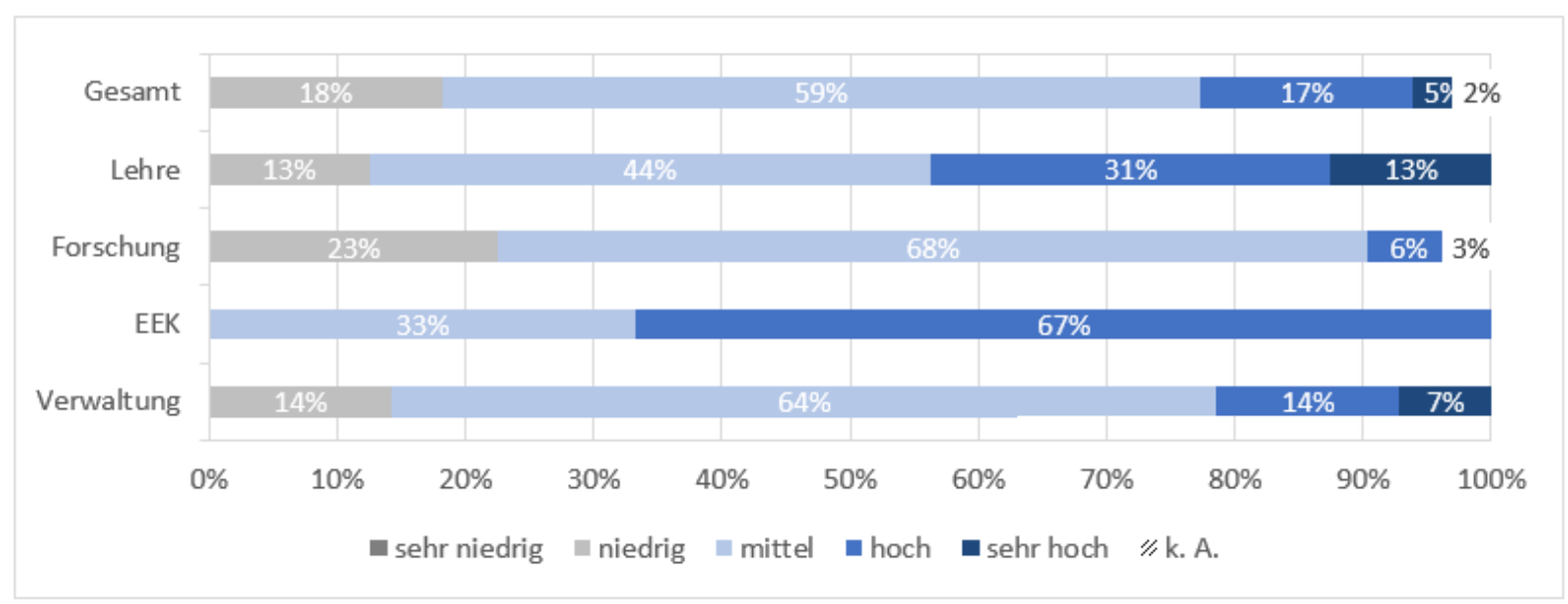

Quelle: WPZ Research Befragung der Projektleiterinnen und -leiter von finanzierten Projekten der HRSM-Ausschreibung 2013, $\mathrm{n}=65$

Im Zuge der Antragsstellung haben $30 \%$ der Projektleiterinnen und -leiter die Unterstützung des BMBWFHelpdesks in Anspruch genommen, 70 \% taten dies nicht. Die Unterstützung entfiel dabei etwa zu gleichen Teilen auf inhaltliche und technische Nachfragen. Die 21 Projektleiterinnen und Projektleiter, welche die Unterstützung des BMBWF in Anspruch nahmen, waren äußerst zufrieden damit. $43 \%$ empfanden die Hilfestellungen als sehr hilfreich, $57 \%$ als eher hilfreich. Der Helpdesk des BMBWF kann daher als Erfolg gesehen werden. 


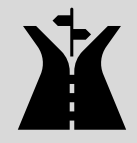

21 \% der nicht-finanzierten Projektleiterinnen und -leiter nahmen die Unterstützung des BMBWFHelpdesk in Anspruch. Das waren um knapp zehn Prozentpunkte weniger als bei den finanzierten Projekten. Keinen Unterschied gab es allerdings bei der Art der Fragestellungen an das BMBWF, wiederum kam es zu einer gleichmäßigen Verteilung zwischen inhaltlichen und technischen Fragen. Die Unterstützung wurde zu $86 \%$ als (eher) hilfreich eingeschätzt.

Im Rahmen der Antragsstellung mussten Bewertungskriterien (unter dem Titel „Indikatorenset und Selbsteinschätzung") ausgefüllt werden. Die inhaltlichen Bewertungskriterien für Lehre und Forschung/EEK umfassten dabei: Konzentrationsgrad in der Lehre, Profitmöglichkeiten für Studierende, Etablierung gemeinsamer Strukturen, nachhaltige Kooperation, institutions- bzw. organisationsübergreifende Integration, Strukturbildungsbeitrag bzgl. begonnener inhaltlicher Neuausrichtungen, Management-Knowhow in/von ForschungsExzellenzstrukturen, Stärkung existierender Exzellenzbereiche, Nachwuchsförderung und Internationalisierungsgrad. Die inhaltlichen Bewertungskriterien für Verwaltungsprojekte umfassten: nachhaltige Effizienzsteigerung, konkrete Synergieeffekte, Nutzen der Innovation für Studierende bzw. Lehrende, Erhöhung der Transparenz von Verwaltungsabläufen, Teilnahme an universitätsübergreifenden (IT-basierten) Verwaltungsnetzen und Innovationen/Optimierungen im IKT-Bereich.

Abbildung 6: Passgenauigkeit/Angemessenheit der Bewertungskriterien („Indikatorenset und Selbsteinschätzung") der Ausschreibung

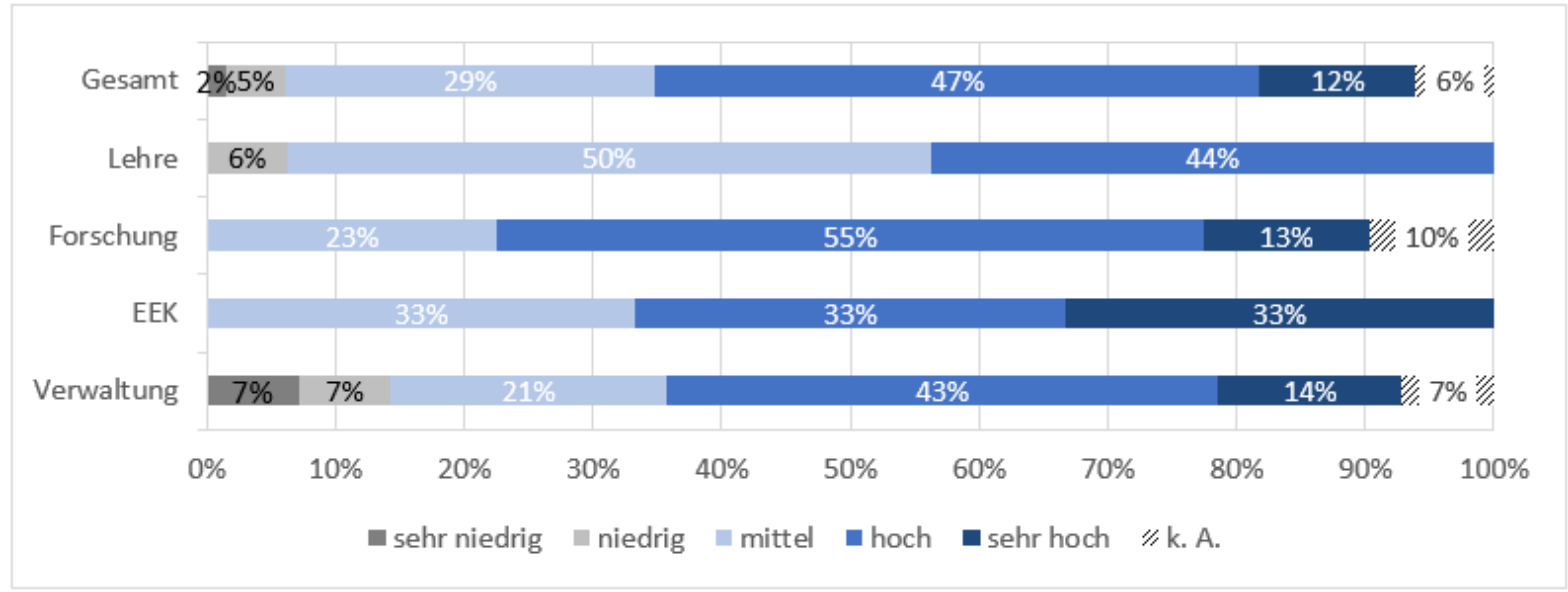

Quelle: WPZ Research Befragung der Projektleiterinnen und -leiter von finanzierten Projekten der HRSM-Ausschreibung 2013, $\mathrm{n}=66$

Die Umfrage zeigt, dass etwas mehr als die Hälfte (59\%) der Projektleiterinnen und Projektleiter die Passgenauigkeit bzw. die Angemessenheit dieser Bewertungskriterien als (sehr) hoch empfanden, $29 \%$ als mittel und $7 \%$ als (eher) niedrig. Je nach inhaltlichem Schwerpunkt des Projekts unterscheidet sich das Antwortverhalten etwas. Bei Lehrprojekten wurde die Passgenauigkeit etwas geringer wahrgenommen, bei Projekten der Entwicklung und Erschließung der Künste - für die nur sehr wenige Antworten vorliegen - etwas höher. Das generell positive Bild wird allerdings über alle inhaltlichen Schwerpunkte hinweg geteilt.

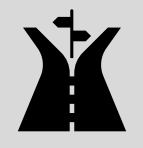

Deutliche Unterschiede zwischen finanzierten und nicht-finanzierten Projekten gab es bei der Wahrnehmung zur Passgenauigkeit bzw. Angemessenheit der Bewertungskriterien der Ausschreibung. Während bei den finanzierten Projekten die Passgenauigkeit bzw. Angemessenheit mit $59 \%$ als (sehr) hoch eingeschätzt wurde, waren es bei den nicht-finanzierten Projekten lediglich $30 \%$. Stattdessen bewertete mit $55 \%$ rund die Hälfte der Befragten die Passgenauigkeit bzw. Angemessenheit lediglich als mittelmäßig. 
Werden die Projekte nach inhaltlichem Schwerpunkt unterschieden, so wurde die Passgenauigkeit bei Projekten im Bereich Lehre - sofern Angaben gemacht wurden - etwas höher eingeschätzt. Für Projekte im Bereich Entwicklung und Erschließung der Künste bzw. im Bereich Verwaltung liegen leider wieder nur sehr geringe Fallzahlen vor.

Abbildung 7: Passgenauigkeit/Angemessenheit der Bewertungskriterien („Indikatorenset und Selbsteinschätzung") der Ausschreibung

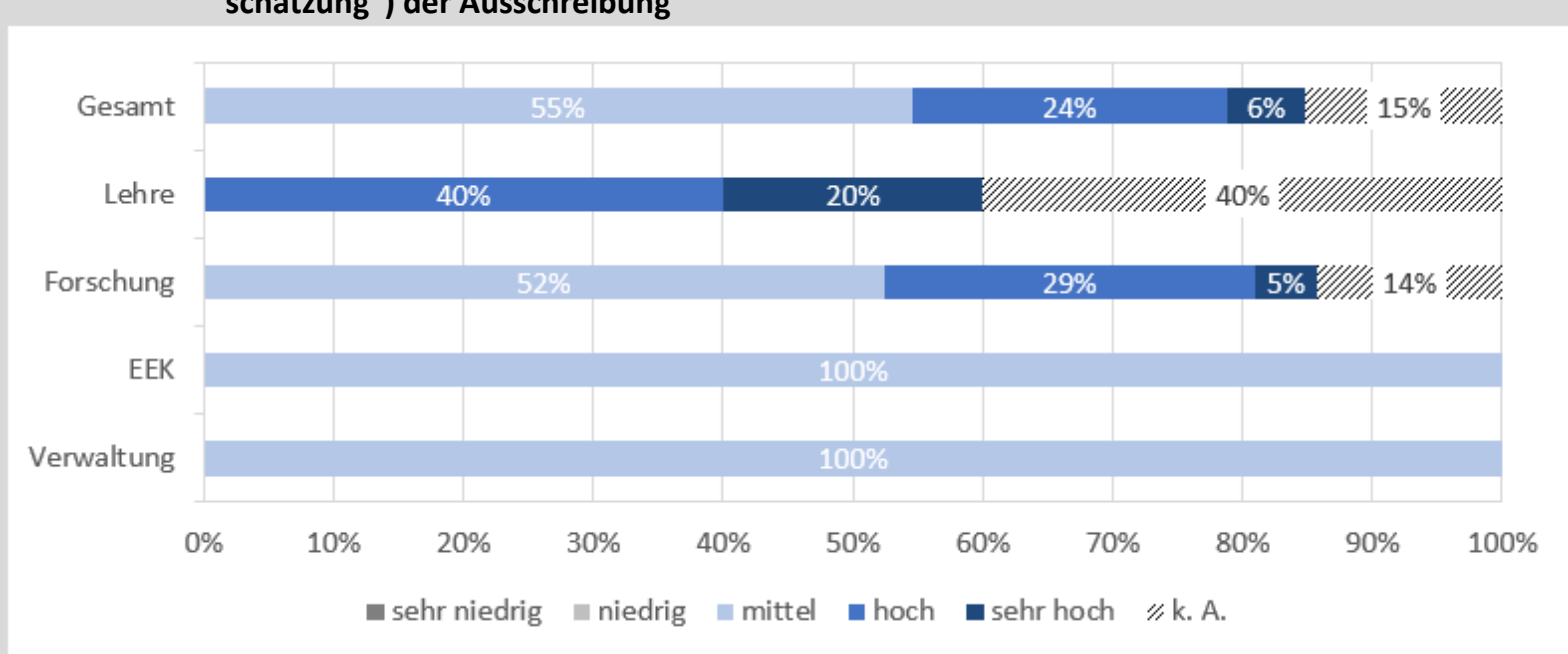

Quelle: WPZ Research Befragung der Projektleiterinnen und -leiter von finanzierten Projekten der HRSM-Ausschreibung 2013, $n=33$

Im Zuge des Antragsverfahrens mussten die Antragstellerinnen und Antragsteller um HRSM 2013 auch einen Bezug zur Leistungsvereinbarung bzw. zum Entwicklungsplan ihrer Universität angeben. Fast drei Viertel (74 \%) der Projektleiterinnen und -leiter von finanzierten Projekten empfanden es als (sehr) leicht, einen solchen Bezug herzustellen. Lediglich $2 \%$ gaben an, dass dies sehr schwierig war.

Abbildung 8: Bezug zur Leistungsvereinbarung 2013-2015 bzw. zum Entwicklungsplan der Universität

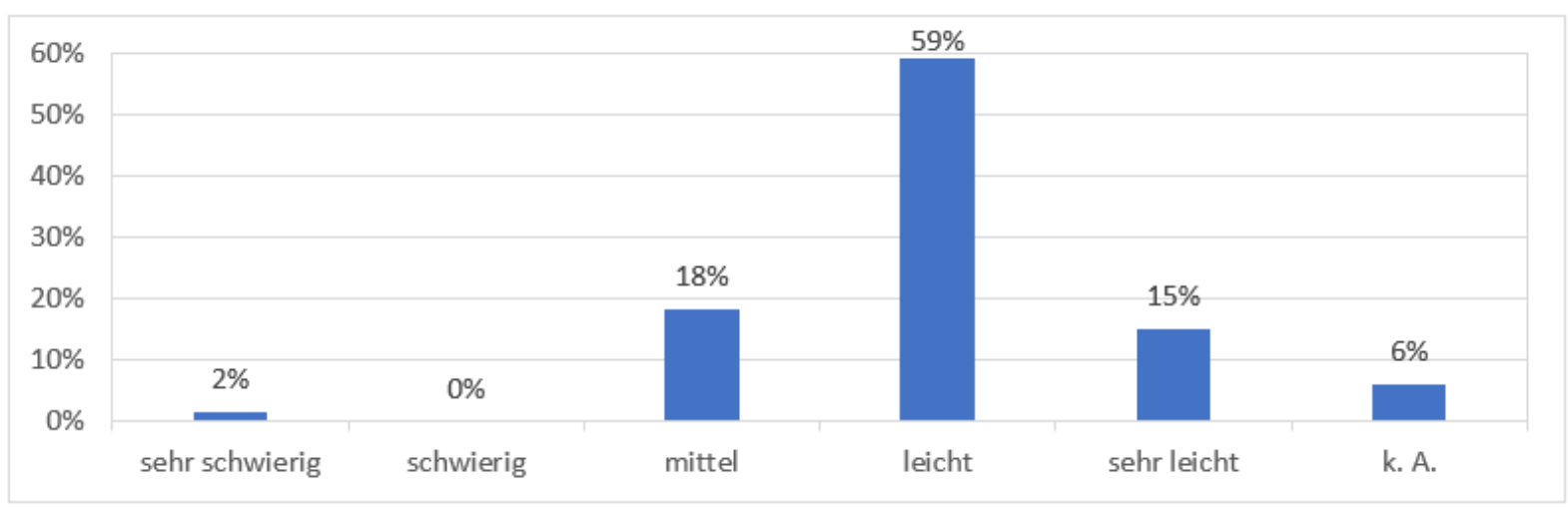

Quelle: WPZ Research Befragung der Projektleiterinnen und -leiter von finanzierten Projekten der HRSM-Ausschreibung 2013, $\mathrm{n}=66$

Im großen Bild deckt sich diese Einschätzung nicht wirklich mit den Kommentaren/Anmerkungen seitens des BMBWF. So wurde bei der Auswertung der Anträge immer wieder angemerkt, dass die Bezüge zu den Leistungsvereinbarungen und den Entwicklungsplänen nicht vollständig nachvollziehbar sind, da eine Verknüpfung mit dem Projekt nur sehr abstrakt bzw. in seltenen Fällen gar nicht herstellbar ist. Angesichts dessen scheint es hier Interpretationsspielräume (bzw. unterschiedliche Sichtweisen) zu geben, inwieweit ein HRSM-Projekt konkrete Anknüpfungen aufweisen soll. 


\subsubsection{Vergabeentscheidung}

Was das Vergabeverfahren der HRSM 2013 betrifft, so zeigt sich in der Online-Befragung, dass die Projektleiterinnen und Projektleiter hier noch Verbesserungspotential sehen. Die relative Mehrheit von $36 \%$ stufte die Transparenz der Vergabe lediglich als mittelmäßig ein. $28 \%$ empfanden sie sogar als (eher) niedrig, $22 \%$ dagegen als (eher) hoch. Die Bestnote vergaben allerdings lediglich $2 \%$ der Projektleiterinnen und -leiter. Die Dauer des Auswahlverfahrens von der Einreichung bis zur Bewilligung sahen die Projektleiterinnen und -leiter dagegen positiver. $54 \%$ beurteilten die Angemessenheit der Dauer als (sehr) hoch, $33 \%$ als mittel und nur $7 \%$ als (sehr) niedrig.

Die Nachvollziehbarkeit der Vergabeentscheidung stuften die Projektleiterinnen und -leiter zu $29 \%$ als (sehr) hoch ein, $38 \%$ sahen sie als mittel an und $20 \%$ sogar als (sehr) niedrig. Offenbar bestehen in diesem Bereich Verbesserungspotentiale für zukünftige Ausschreibungen. Die Vergabe war auf Basis des Vorschlags einer Auswahlkommission, bestehend aus zwei Personen seitens des BMBWF und jeweils einer Person seitens des BMF und der uniko, getroffen worden.

\section{Abbildung 9: Vergabeverfahren}

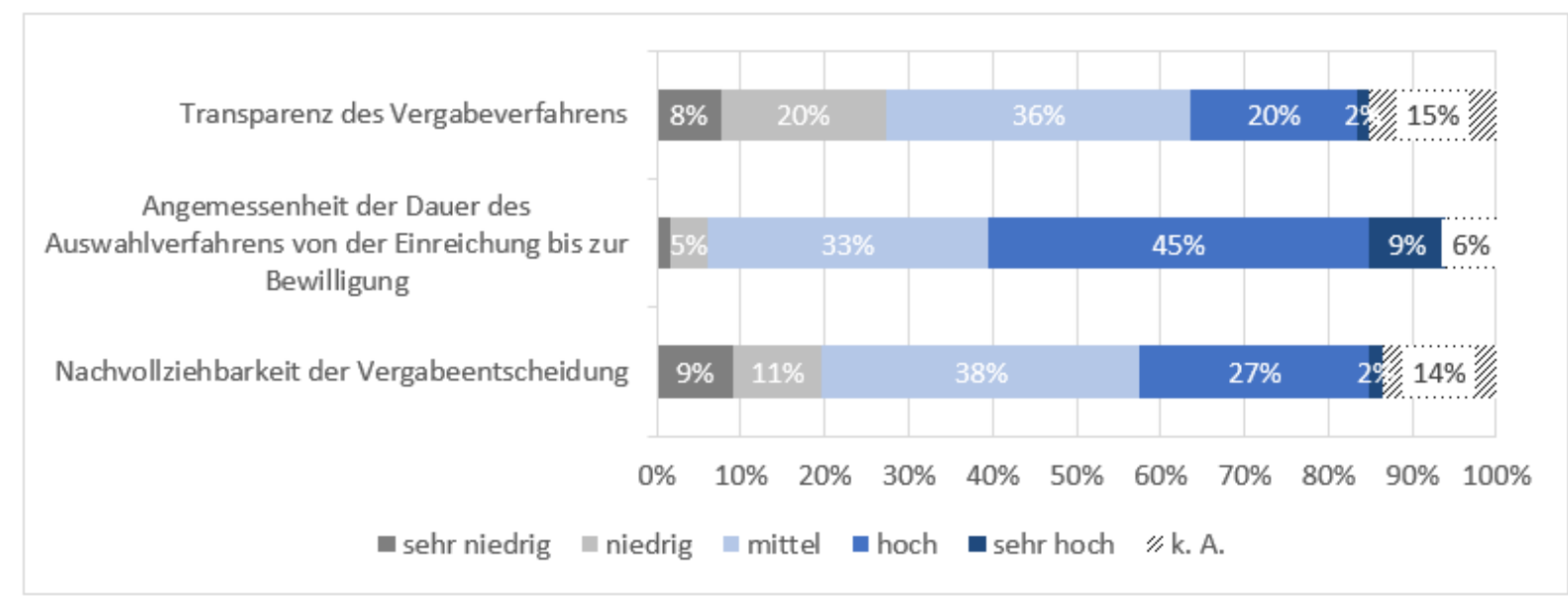

Quelle: WPZ Research Befragung der Projektleiterinnen und -leiter von finanzierten Projekten der HRSM-Ausschreibung 2013, $\mathrm{n}=66$

Auch in der freien Beantwortung der Frage, wie der Verfahrensablauf in Zukunft verbessert werden könnte, wünschten sich einige Projektleiterinnen und -leiter von HRSM-finanzierten Projekten der Ausschreibung 2013 eine höhere Transparenz. Einige Anmerkungen hierzu sind in der nachfolgenden Box festgehalten.

\section{Statements der Befragten:}

"Mehr Transparenz, Punkte vergeben je Kriterium, breitere Fachjury"

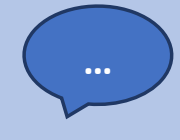

"Erhöhung der Nachvollziehbarkeit und Transparenz des Vergabeverfahrens"

„Aus ProjektleiterInnen-Sicht wäre es wünschenswert gewesen, etwas länger für die Antragstellung Zeit zu haben bzw. beim Antrag auch einen detaillierteren Projektplan (nach einem PM-Standard) mit abzugeben (einzufordern). Dabei wäre es gut, wenn nicht nur das Abgeben von Antragstexten, sondern auch das Hochladen von Gantt-Charts (oder Projektdateien) unterstützt wäre."

"Auflagen" in die Vergabeentscheidung sollten im Vorfeld mit den Antragstellern abgeklärt werden („,Was erwartet sich das Ministerium"); nicht alles ist machbar, was sich ministerielle Entscheidungsträger vorstellen; Auflagen werden in weiterer Folge zu einer Bürde eines Projekts, zumal eine solche Entscheidung für keine der Parteien (eigenes HRSM-Projekt sowie Verantwortliche eines anderen HRSM-Projekts) nachvollziehbar, transparent ist." 


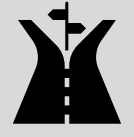

Dem Vergabeverfahren standen die befragten, nicht durch HRSM-Mittel finanzierten Personen der Ausschreibung 2013 noch kritischer gegenüber, was naturgemäß u.a. daran liegt, dass diese Personen keine Finanzierung erhalten haben. Keine bzw. keiner der nicht-finanzierten Projektleiterinnen und Projektleiter schätzte die Transparenz des Vergabeverfahrens als (sehr) hoch ein. Stattdessen bewerteten $38 \%$ die Transparenz als mittel und $53 \%$ als (sehr) niedrig. Ganz ähnliche Werte zeigen sich bei der Nachvollziehbarkeit der Vergabeentscheidung, auch diese wurde von $47 \%$ als (sehr) niedrig eingeschätzt. Die Angemessenheit der Dauer des Auswahlverfahrens schnitt dagegen besser ab. Die Leiterinnen und Leiter von nicht-finanzierten Projekten bewerteten sie zu $31 \%$ als (sehr) hoch und zu $38 \%$ als mittelmäßig. Lediglich $6 \%$ empfanden sie als (sehr) niedrig.

\section{Abbildung 10: Vergabeverfahren}

Transparenz des Vergabeverfahrens

$25 \% \quad 28 \% \quad 38 \% \quad$ 浐 $9 \%$

Angemessenheit der Dauer des

Auswahlverfahrens von der Einreichung bis zur Bewilligung

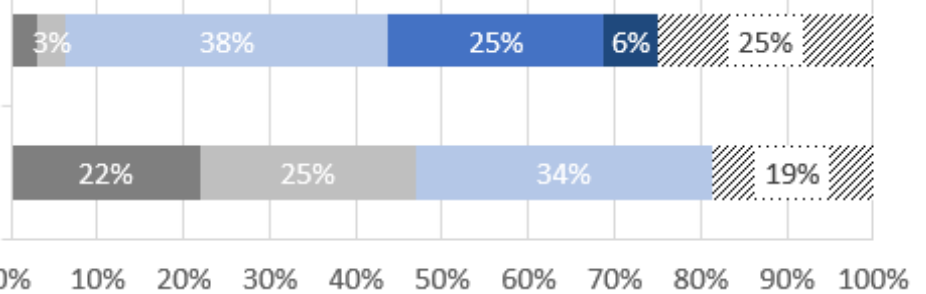

Nachvollziehbarkeit der Vergabeentscheidung

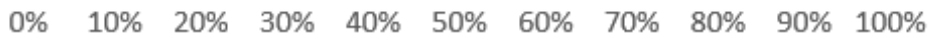

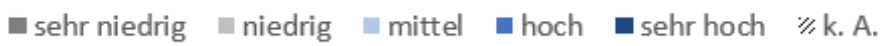

Quelle: WPZ Research Befragung der Projektleiterinnen und -leiter von nicht-finanzierten Projekten der HRSM-Ausschreibung 2013, $n=32$

Dieses kritische Urteil der Leiterinnen und Leiter von nicht-finanzierten Projekten zeigt sich auch bei der Frage, ob es für sie nachvollziehbar war, warum bzw. aufgrund welcher Kriterien ihr Projektantrag abgelehnt wurde. $72 \%$ verneinten diese Aussage (eher) und lediglich $12 \%$ stimmten ihr (eher) zu.

Abbildung 11: Nachvollziehbarkeit, warum bzw. aufgrund welcher Kriterien Ihr Projektantrag abgelehnt wurde

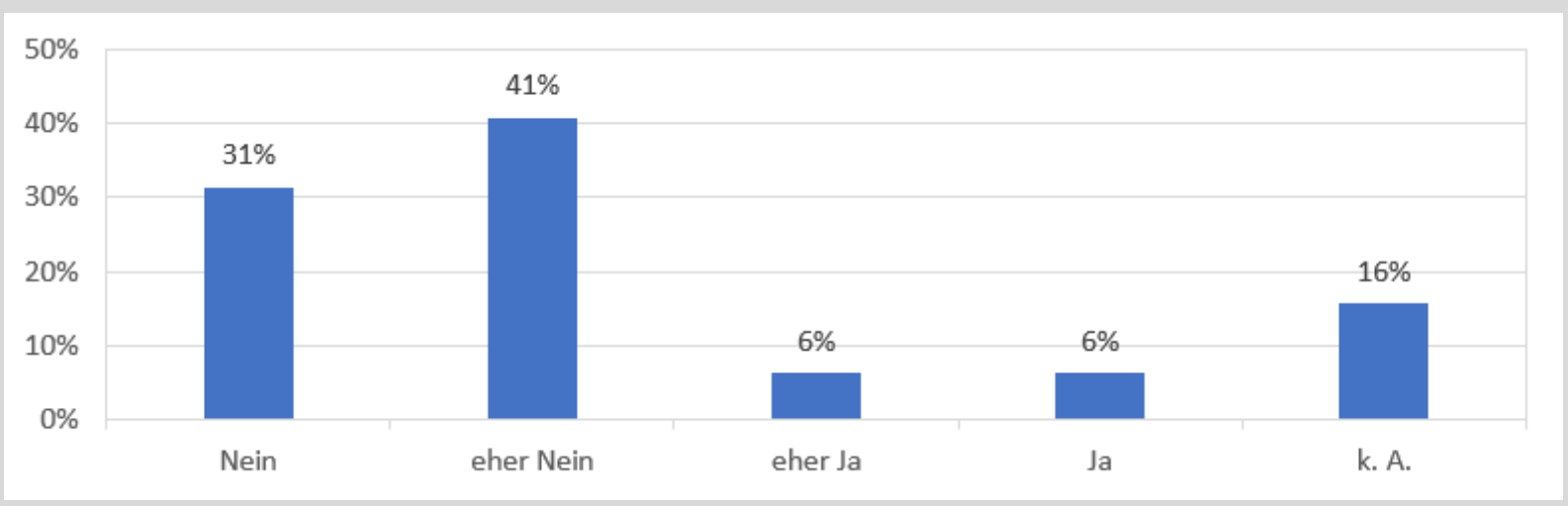

Quelle: WPZ Research Befragung der Projektleiterinnen und -leiter von nicht-finanzierten Projekten der HRSM-Ausschreibung 2013, $n=32$

Dieses Stimmungsbild spiegelt sich auch in der Beantwortung der Frage, wie das Vergabeverfahren verbessert werden könnte, wider. Zahlreiche Befragten wünschten sich eine verbesserte Transparenz und Nachvollziehbarkeit der Entscheidung des BMBWF. Ausgewählte Verbesserungsvorschläge sind hierzu in der nachfolgenden Box dargestellt. 


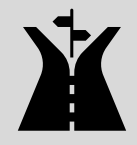

Statements der Befragten:

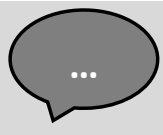

„Kommunikation der Kriterien, warum das Projekt abgelehnt wurde, und Transparenz in der Vergabe"

"Ich möchte die Gutachten auch bei Ablehnung sehen dürfen."

„ICh würde eine Beurteilung der Projekte durch ein/mehrere Expertengremium/en mit Expert/innen außerhalb des Ministeriums begrüßen. Alternativ würde es auch möglich sein, klare inhaltliche Vorstellungen bei der Ausschreibung zu formulieren - dann wären Entscheidungen allenfalls besser nachvollziehbar."

„Mitteilung der Ablehnungsgründe. Wenn diese allenfalls der Universität mitgeteilt wurden, wurden sie jedenfalls nicht weiter kommuniziert."

"Klarere Zielvorgaben und Abstimmung"

Die Auswertung der Projektanträge zeigt folgendes Bild: Insgesamt wurden 218 Projekte im Rahmen der HRSMAusschreibung 2013 eingereicht, 83 (38\%) davon wurden schließlich genehmigt und erhielten eine Finanzierung seitens des BMBWF. Wie Abbildung 12 veranschaulicht, wurden insgesamt 140 Anträge (64\%) im Bereich Forschung/EEK gestellt, dahinter folgten insgesamt 45 Anträge (21\%) in der Lehre und 33 Anträge (40 \%) in der Verwaltung. Daraus ergeben sich folgende Bewilligungsquoten: 31 \% im Bereich Forschung/EEK, 38 \% im Bereich Lehre und $67 \%$ im Bereich Verwaltung.

\section{Abbildung 12: Verhältnis zwischen finanzierten und nicht-finanzierte Projekten nach Bereichen (in absoluten Zahlen)}

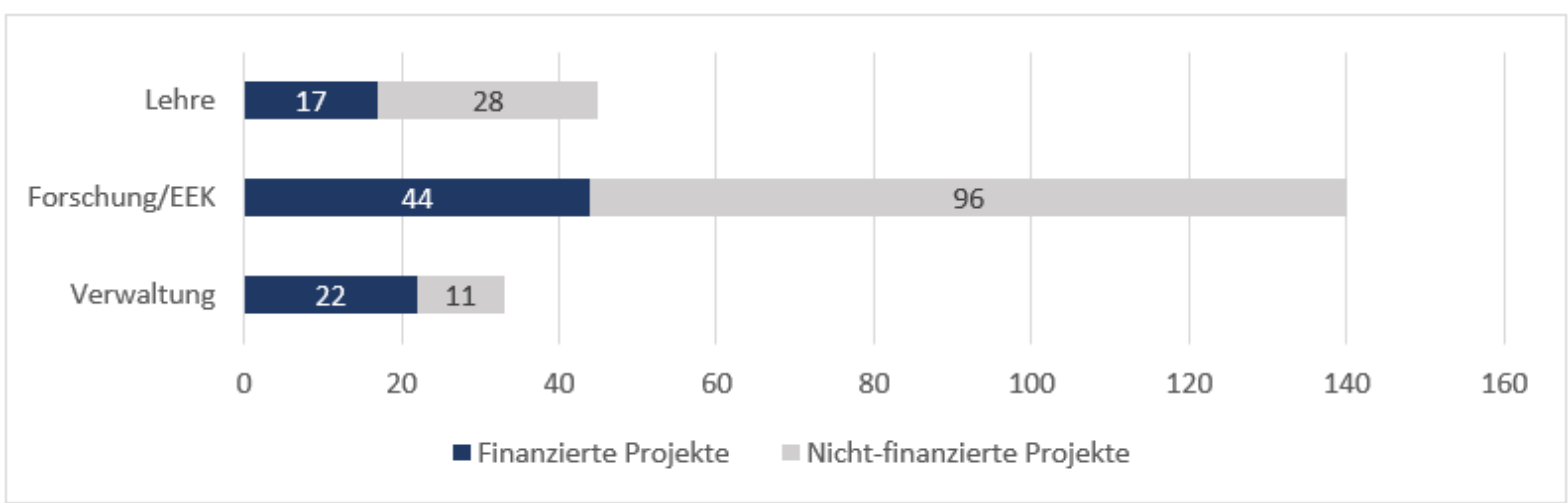

Quelle: BMBWF, eigene Berechnungen; $\mathrm{n}=218$

Bemerkenswert ist, dass drei Volluniversitäten, nämlich die Universität Wien, die Universität Graz und die Universität Innsbruck, im Vergleich zu den anderen Universitäten die niedrigsten Bewilligungsquoten aufzeigen. Dies mag insbesondere darauf zurückzuführen sein, dass diese Universitäten (im Vergleich zu den anderen Universitäten) eine relativ hohe Anzahl an Anträgen gestellt haben. 
Abbildung 13: Bewilligungsquote nach Universitäten und Bereich, sowie nach Universitätstyp

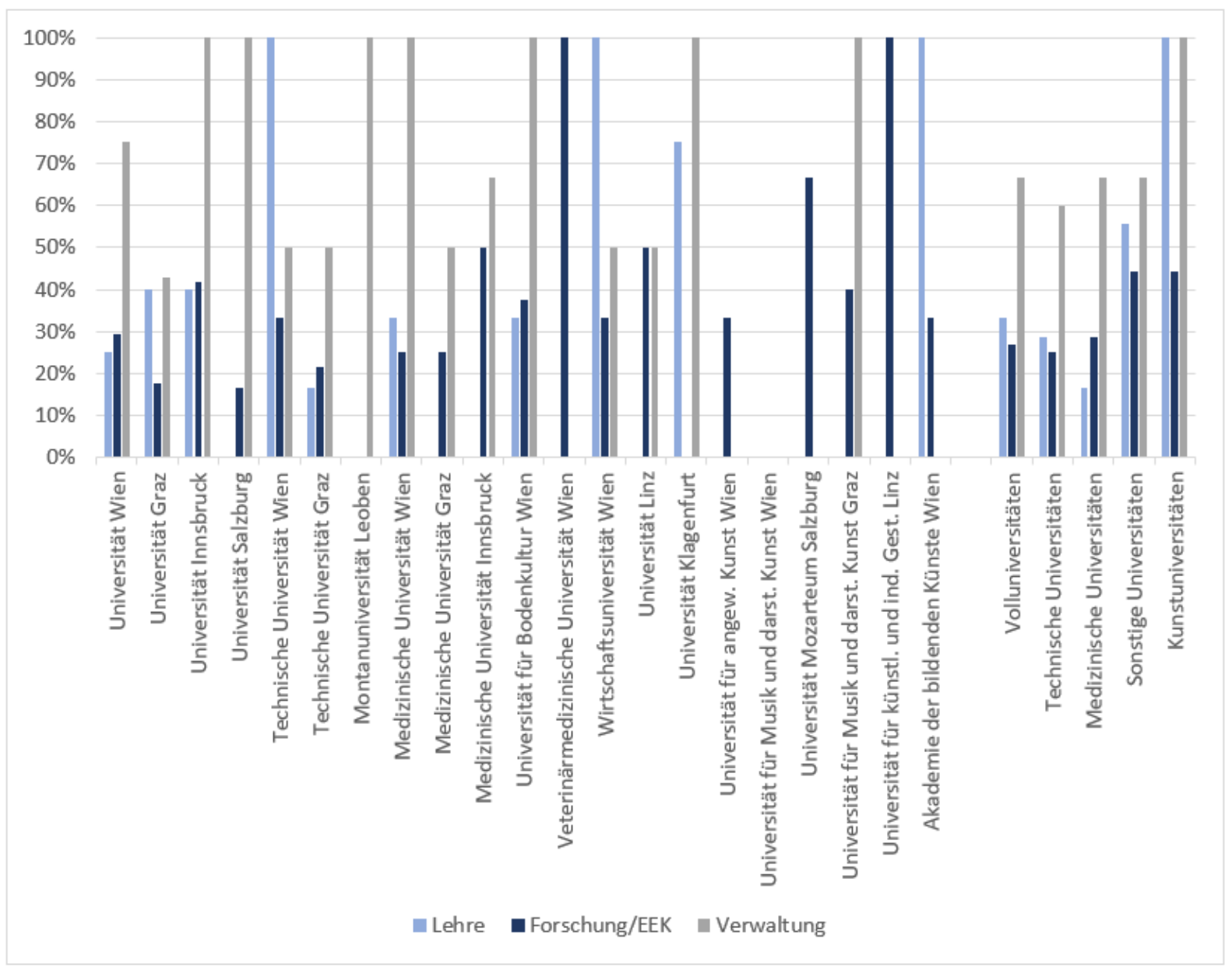

Quelle: BMBWF, eigene Berechnungen; $n=218$

Darüber hinaus zeigt sich, dass sich einige Universitäten - die unterschiedlichen Schwerpunkte der HRSMAusschreibung betreffend - durchaus selektiv um die HRSM-Mittel 2013 beworben haben (siehe Abbildung 13). So haben die Montanuniversität Leoben und die Kunstuniversitäten (mit Ausnahme der Akademie der bildenden Künste Wien) keine Anträge im Bereich Lehre gestellt. Im Bereich Forschung/EEK haben dagegen - bis auf die Universität für Musik und darstellende Kunst Wien - alle Universitäten Anträge eingereicht. Im Bereich Verwaltung zeigt sich, dass bis auf die Universität für Musik und darstellende Kunst Graz keine Kunstuniversität einen Antrag gestellt hat. Offenbar unterscheiden sich Kunstuniversitäten diesbezüglich von anderen Universitätstypen. Auch die Veterinärmedizinische Universität Wien hat im Bereich Verwaltung keinen Antrag gestellt, allerdings war diese in mehreren (finanzierten) Verwaltungsprojekten als Kooperationspartnerin involviert. Ferner kann in diesem Kontext festgehalten werden, dass alle Projektanträge der Universität Innsbruck, der Universität Salzburg, der Montanuniversität Leoben, der Medizinischen Universität Wien, der Universität für Bodenkultur sowie der Universität für Musik und darstellende Kunst Graz im Bereich der Verwaltung genehmigt wurden. 


\subsection{Zur Umsetzung}

In weiterer Folge werden die Umsetzung bzw. der Umsetzungserfolg der im Rahmen der HRSM-Ausschreibung 2013 finanzierten Projekte analysiert. Im Fokus stehen hierbei Projektcharakteristika, die Finanzierung, thematische Schwerpunkte sowie das Kooperationsverhalten der finanzierten bzw. beteiligten Akteure.

\subsubsection{Finanzierte Projekte und ihre Charakteristika}

Inhaltlich sind die durch die HRSM-Ausschreibung 2013 finanzierten Projekte sehr breit gefächert und reichen diese von der Schaffung einheitlicher Aufnahmeverfahren für Humanmedizin bzw. Zahnmedizin, über die Strukturförderung der Quantenforschung in Wien im Rahmen des Vienna Center for Quantum Science and Technology (VCQ), welche durch die Anschaffung kostenintensiver Forschungsinfrastruktur realisiert wurde, bis hin zum Campusmanagement an Österreichischen Universitäten, welches etwa die wesentliche Verbesserung der Usability insbesondere für Studierende und Lehrende zum Ziel hatte. Die Förderung der Forschungsinfrastruktur war ein explizites Ziel der HRSM-Ausschreibung 2013. Angesichts dessen wurden auch insgesamt 39 Kooperationsprojekte (47\%), welche die Stärkung der Forschungsinfrastruktur zum Ziel hatten, finanziert (BMBWF, 2014).

Betrachtet man die Verteilung der verschiedenen Projekttypen auf die Universitäten, so zeichnet sich folgendes Bild: Projekte im Bereich Forschung/EEK machen bei allen Universitätstypen den größten Anteil aus. Dieser Anteil reicht von $45 \%$ (14 finanzierte Projekte) bei Volluniversitäten bis zu $63 \%$ (fünf finanzierte Projekte) bei Kunstuniversitäten. In den Bereichen Lehre und Verwaltung unterscheidet sich die Bedeutung nach Universitätstyp zudem teils stark. Während bei den Medizinischen Universitäten nur $8 \%$ der Projekte (zwei finanzierte Projekte) auf den Bereich Lehre entfallen, sind es bei den Sonstigen Universitäten $29 \%$ (fünf finanzierte Projekte). Ebenso unterscheidet sich der Anteil der Verwaltungsprojekte je Universitätstyp. Während die Kunstuniversitäten nur ein einziges Verwaltungsprojekt (13\% aller Projekte) durchgeführt haben, sind es bei den Medizinischen Universitäten vier (31\%).

\section{Abbildung 14: Finanzierte Projekte nach Universitätstyp (in Prozent)}

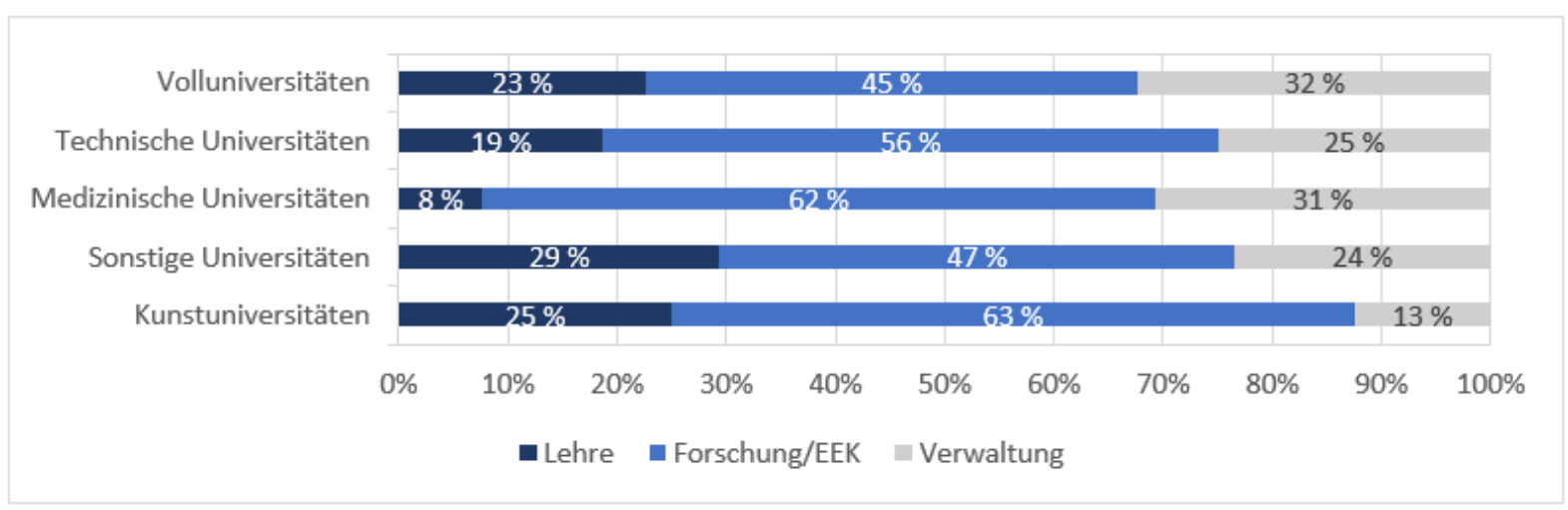

Quelle: BMBWF, eigene Berechnungen; $n=83$

Im Durchschnitt weisen die Volluniversitäten - über alle Bereiche hinweg - mit acht finanzierten Projekten pro Universität die meisten bewilligten bzw. finanzierten Projekte in der HRSM-Ausschreibung 2013 auf. Die Universität Innsbruck verfügt mit 12 finanzierten Projekten über die höchste Anzahl an bewilligten Projekten. Demgegenüber konnten die Kunstuniversitäten mit durchschnittlich 1,8 finanzierten Projekten eine nur sehr geringe Anzahl an Projekten einwerben. Andere Universitäten wie die Montanuniversität Leoben (mit zwei Anträgen), die Universität für angewandte Kunst Wien (mit drei Anträgen) und die Universität für künstlerische und industrielle Gestaltung Linz (mit einem Antrag) konnten Mittel für jeweils ein Projekt einwerben. Sonstige Universitäten weisen im Durchschnitt drei bewilligte bzw. finanzierte Projekte auf, Medizinische und Technische Universitäten im Durchschnitt jeweils vier finanzierte Projekte. Die Verteilung der beantragten und finanzierten Projekte auf die einzelnen Universitäten unterstreicht den offensichtlichen Zusammenhang zwischen der Größe der Universität und der Anzahl an Projekten. Die großen Volluniversitäten mit vielen Forschungsschwerpunkten und Instituten haben entsprechend auch mehr Anträge gestellt. 
Abbildung 15: Anzahl der finanzierten Projekte nach Universitäten und Bereich, sowie durchschnittliche Anzahl nach Universitätstyp (in absoluten Zahlen)

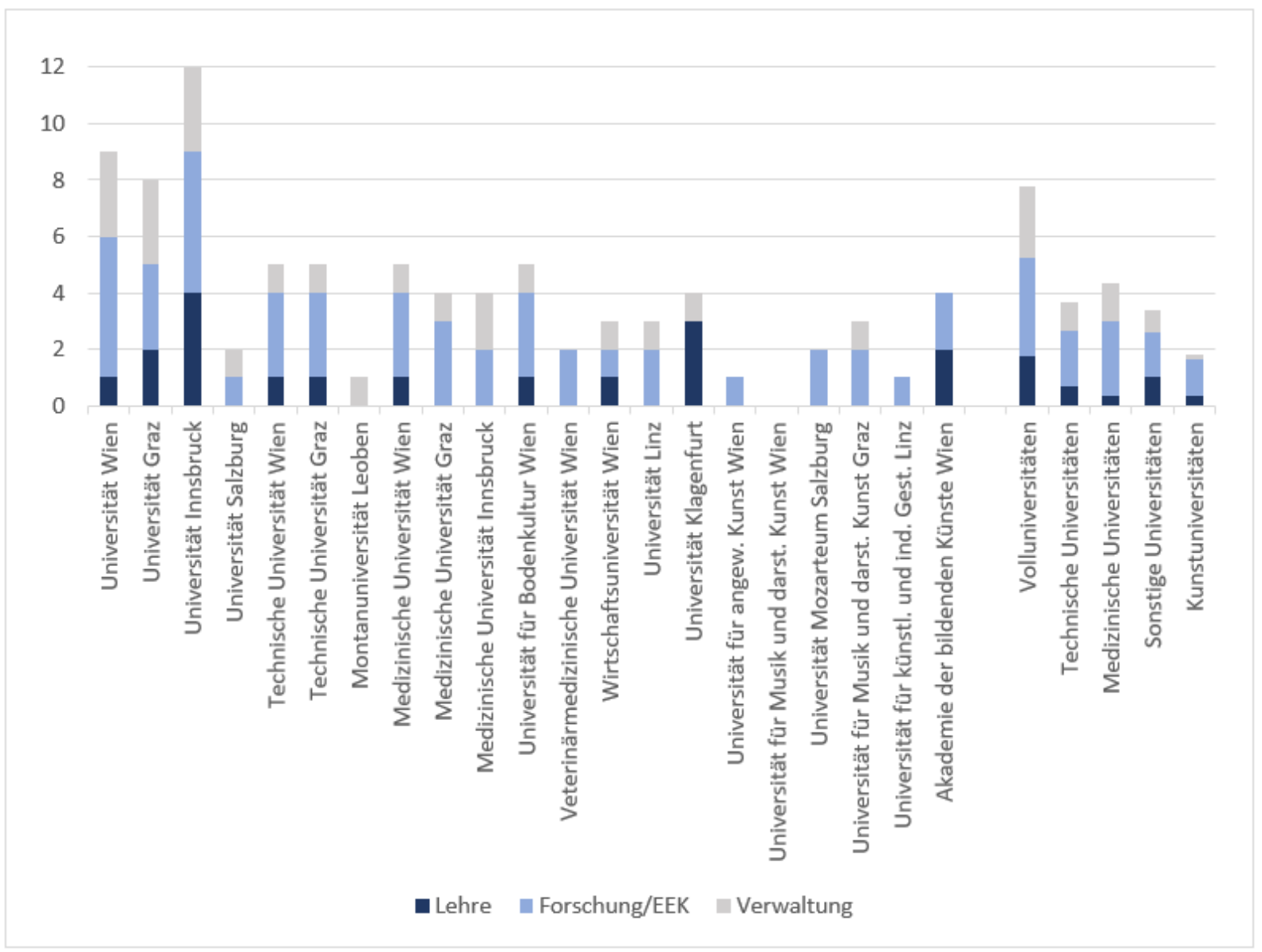

Quelle: BMBWF, eigene Berechnungen; $\mathrm{n}=83$

\subsubsection{Finanzierung}

Im Rahmen der HRSM-Ausschreibung 2013 hat das BMBWF generell maximal ein Drittel der gesamten Projektkosten finanziert. Geht man vom Prozess der Antragstellung aus, so wurden insgesamt 218 Projekte mit einem Volumen von 159,11 Mio. Euro beantragt, wovon 83 Projekte mit einem Antragsvolumen in Höhe von 73,25 Mio. Euro bewilligt wurden, tatsächlich seitens des BMBWF finanziert wurden 63 Mio. Euro. Hintergrund ist, dass im Zuge des Bewilligungsprozesses bei 46 Projekten (das sind $55 \%$ aller bewilligten Projekte) das Projektvolumen im Zuge des Auswahlverfahrens gekürzt wurde.

Blickt man auf die Kostendisziplin, d.h. inwieweit wurde der Kostenrahmen bei der Umsetzung der Projekte eingehalten, so zeigt sich, dass 64 Projekte (das sind $77 \%$ aller bewilligten Projekte) nach Projektabschluss höhere Kosten aufweisen (im Durchschnitt $10 \%$ ), als sie ursprünglich beantragt haben. Die Bandbreite der Überschreitungen liegt dabei zwischen 0,01 \% und $85 \%$. Insgesamt wurden bei fast allen Verwaltungsprojekten (bei insgesamt 19 Projekten bzw. $86 \%$ ) die anberaumten Projektkosten überschritten. Der Durchschnitt der Überschreitung liegt dabei bei $17 \%$. Etwas weniger oft wurden die Projektkosten im Bereich Lehre (71 \%) und Forschung/EKK (75 \%) überschritten. Auch hier variiert das Ausmaß der Überschreitung: Fielen die Projektkosten in der Lehre durchschnittlich um $13 \%$ höher aus, so betrug die Überschreitung im Bereich Forschung/EEK im Durchschnitt nur etwa $1 \%$.

Insgesamt haben damit neun Projekte (11\%) ihr ursprüngliches Kostenziel exakt erreicht; d.h. die Kosten entsprachen dem beantragten Volumen. Andere neun Projekte (11\%) haben die beantragte Projektsumme gar unterschritten, im Durchschnitt um $14 \%$. Festzuhalten ist, dass die Kostenüber- bzw. -unterschreitungen die (Inkind-)Leistungen der Kooperationspartnerinnen und -partner betrafen. Bei den vom BMBWF zugesicherten 
Mitteln handelte es sich hingegen um nicht überschreitbare Obergrenzen, die im Falle der Unterschreitung der Projektkosten auf höchstens ein Drittel reduziert wurden.

Insgesamt wurden im Rahmen der Anschubfinanzierung der HRSM-Ausschreibung 2013 für Kooperationen der Universitäten in Lehre und Forschung/Entwicklung und Erschließung der Künste sowie der Verwaltung Projekte in der Höhe von 63 Mio. finanziert. Dieser Wert liegt damit - wie bereits erwähnt - rund zehn Mio. Euro unter den ursprünglich beantragen Finanzierungsmitteln. Die Analyse der Daten zeigt (siehe Abbildung 16), dass die Volluniversitäten mit rund 26,9 Mio. Euro (das entspricht etwa $43 \%$ der gesamten HRSM-Mittel dieser Ausschreibung) die meisten Finanzierungsmittel einwerben konnten, gefolgt von den Medizinischen Universitäten mit 12,95 Mio. Euro (21\%), den Sonstigen Universitäten mit 10,92 Mio. Euro (17\%), den Technischen Universitäten mit 9,46 Mio. Euro (15\%) und den Kunstuniversitäten mit 2,77 Mio. Euro (4 \%).

Betrachtet man die beantragten Mittel auf Ebene der einzelnen Universitäten, so hat die Universität Wien mit insgesamt 16 beantragen Projekten mit einem Volumen von 22,3 Mio. Euro die meisten Mittel beantragt. Davon entfielen 1,61 Mio. Euro (7 \%) auf den Bereich Lehre, 17,9 Mio. Euro (80 \%) auf den Bereich Forschung/EEK und 2,79 Mio. Euro (13\%) auf den Bereich Verwaltung. Der Universität Wien folgt die Medizinische Universität Wien. Die Medizinische Universität Wien hat mit einer Summe von 21,86 Mio. Euro die zweitmeisten Mittel beantragt. Davon entfielen 6,49 Mio. Euro (30\%) auf den Bereich Lehre, 15,18 Mio. Euro (69\%) auf den Bereich Forschung/EEK und rund 191.844 Euro (1\%) auf den Bereich Verwaltung. Nach Universitätstyp haben die Volluniversitäten und die Medizinischen Universitäten die meisten Mittel beantragt, die Kunstuniversitäten hingegen die geringste Summe. Die Höhe der beantragten Mittel reicht dabei von 5.000 Euro bis 4,55 Mio. Euro pro Projekt.

Abbildung 16: Beantragte Vergabesumme aller gestellten Projektanträge nach Universität und Bereich, sowie Durchschnitt nach Universitätstyp (in Mio. Euro)

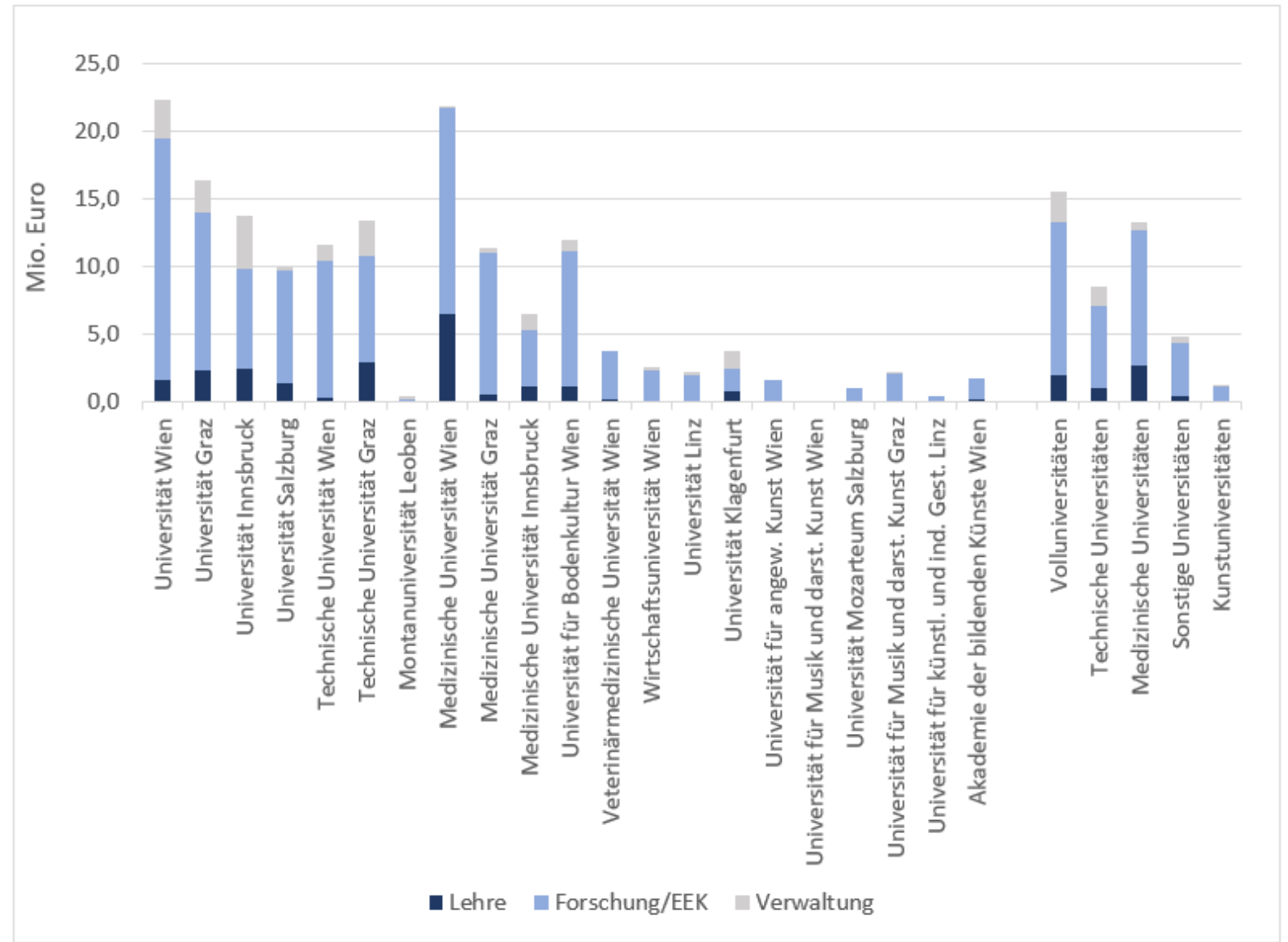

Quelle: BMBWF, eigene Berechnungen; $n=218$ 
Betrachtet man darüber hinaus, wie sich die beantragten Mittel auf die einzelnen Bereiche verteilen, so zeigt sich, was die Antragstellung betrifft, dass insgesamt im Bereich Lehre 21,86 Mio. Euro (14\% der beantragten Mittel), im Bereich Forschung 119,26 Mio. Euro (75 \%) und im Bereich Verwaltung 17,98 Mio. Euro (11\%) angefragt wurden. Die Kunstuniversitäten stellen eine Ausnahme dar. So haben diese 6,65 Mio. Euro (94\% der Mittel) im Bereich Forschung/EEK und jeweils rund 3 \% (237.700 bzw. 205.333 Euro) im Bereich Lehre und Verwaltung beantragt. Zusammenfassend kann somit festgehalten werden, dass drei Viertel der beantragten/ angefragten Mittel auf den Bereich Forschung/EEK entfallen.

Von den insgesamt 159,11 Mio. Euro, welche innerhalb der Projektanträge für eine Finanzierung von 218 Projekten beantragt wurden, hat das BMBWF 83 Projekte mit einer Vergabesumme in der Höhe von 63 Mio. Euro genehmigt. Die genehmigten Mittel gingen zu 8 \% (5,17 Mio. Euro) an die Lehre, zu 68 \% (42,83 Mio. Euro) an die Forschung/EEK und zu $24 \%$ (15,0 Mio. Euro) an die Verwaltung. Die Unterschiede in der Verteilung der beantragten und der tatsächlich vergebenen Mittel auf die Bereiche weisen darauf hin, dass Verwaltungsprojekte überdurchschnittlich oft genehmigt wurden.

Was die bewilligten Finanzierungsmittel je Universität betrifft, konnte die Universität Wien bei neun finanzierten Projekten mit insgesamt 10,39 Mio. Euro die meisten Mittel akquirieren. Die wenigsten Mittel konnte die Universität für angewandte Kunst Wien mit 132.451 Euro und die Montanuniversität Leoben mit 268.000 Euro mit nur jeweils einem finanzierten Projekt akquirieren.

Die Vergabesumme des BMBWF für finanzierte Projekte reichte von 49.429 Euro bis 3,5 Mio. Euro. Das „teuerste“ Projekt fand an der Medizinischen Universität Wien statt - mit einem Gesamtfinanzierungsvolumen in der Höhe von 12,31 Mio. Euro war dies der „Interdisziplinäre translationale Hirnforschungscluster (ITHC) mit Hochfeld $M R^{\prime}$, gefolgt vom „Climate Change Center Austria“ an der Universität Innsbruck mit einem Gesamtfinanzierungsvolumen von 10,68 Mio. Euro. Demgegenüber stellte das Projekt „Von Ideen zu Projekten“ an der Universität Linz mit einem Volumen von 160.168 Euro das Projekt mit den geringsten Kosten dar.

Abbildung 17: Vergabesumme (BMBWF) der finanzierten Projekte nach Universität und Bereich, sowie Durchschnitt nach Universitätstyp (in Mio. Euro)

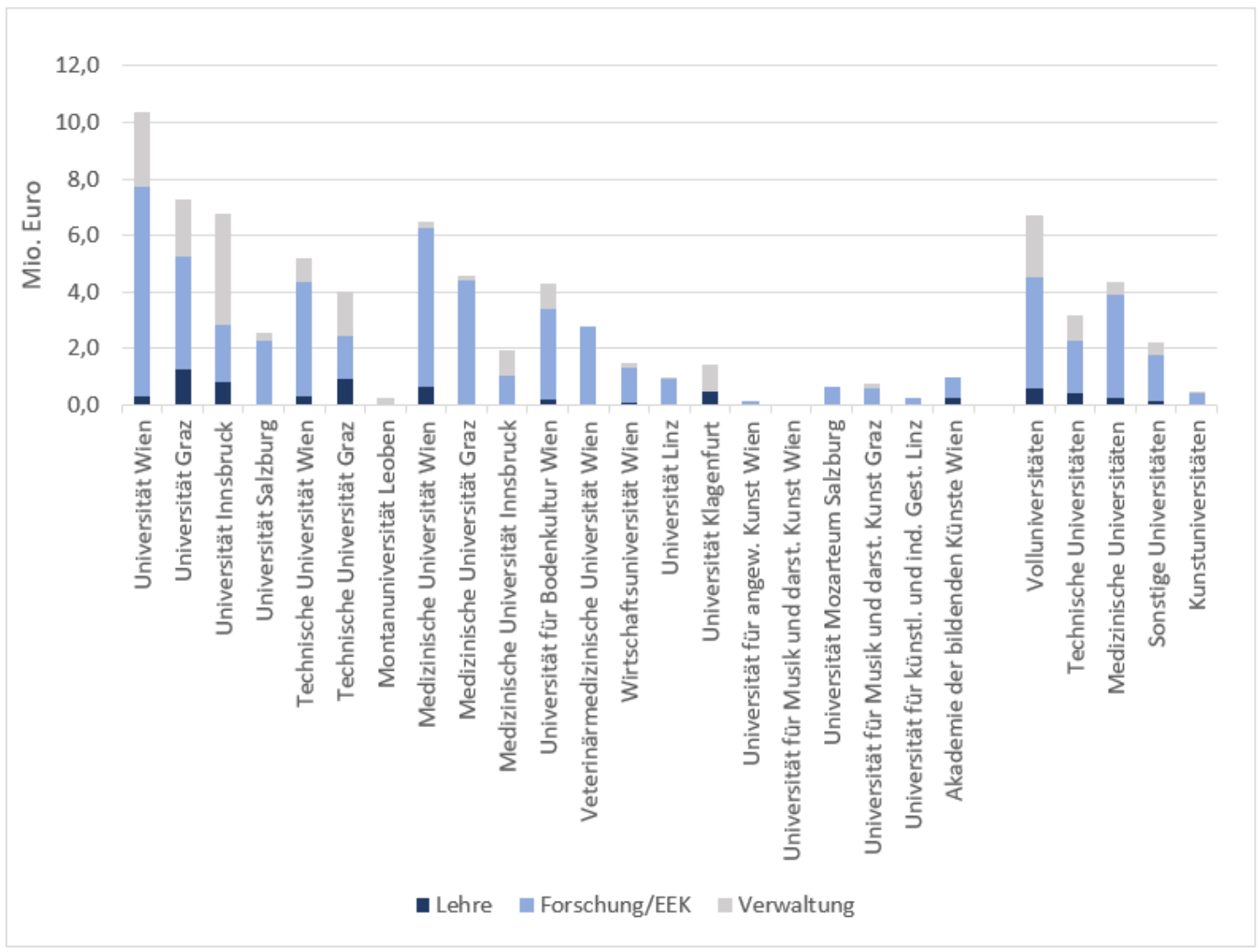

Quelle: BMBWF, eigene Berechnungen; $n=83$ 
Insgesamt gesehen weisen die Volluniversitäten und die Medizinischen Universitäten im Durchschnitt mit 3,23 Mio. Euro bzw. 3,20 Mio. Euro die höchsten Gesamtkosten pro Projekt auf. Auf dem dritten Platz liegen die Technischen Universitäten mit durchschnittlichen Kosten von 2,34 Mio. Euro pro Projekt, gefolgt von den Sonstigen Universitäten mit 2,22 Mio. Euro. Im Gegensatz dazu weisen die Kunstuniversitäten viel geringere Volumina mit durchschnittlich 754.789 Euro pro Projekt auf. Betrachtet man die durchschnittlichen Kosten pro Projekt und pro Universität, so zeigt sich, dass die Medizinische Universität Wien mit durchschnittlich 4,52 Mio. Euro die höchsten Kosten pro Projekt aufweist, gefolgt von der Veterinärmedizinischen Universität Wien mit durchschnittlich 4,27 Mio. Euro Kosten pro Projekt.

Abbildung 18: Durchschnittliche Gesamtkosten/beantragte Mittel/bewilligte Mittel der finanzierten Projekte in Mio. Euro nach Universität sowie Universitätstyp (in Mio. Euro)

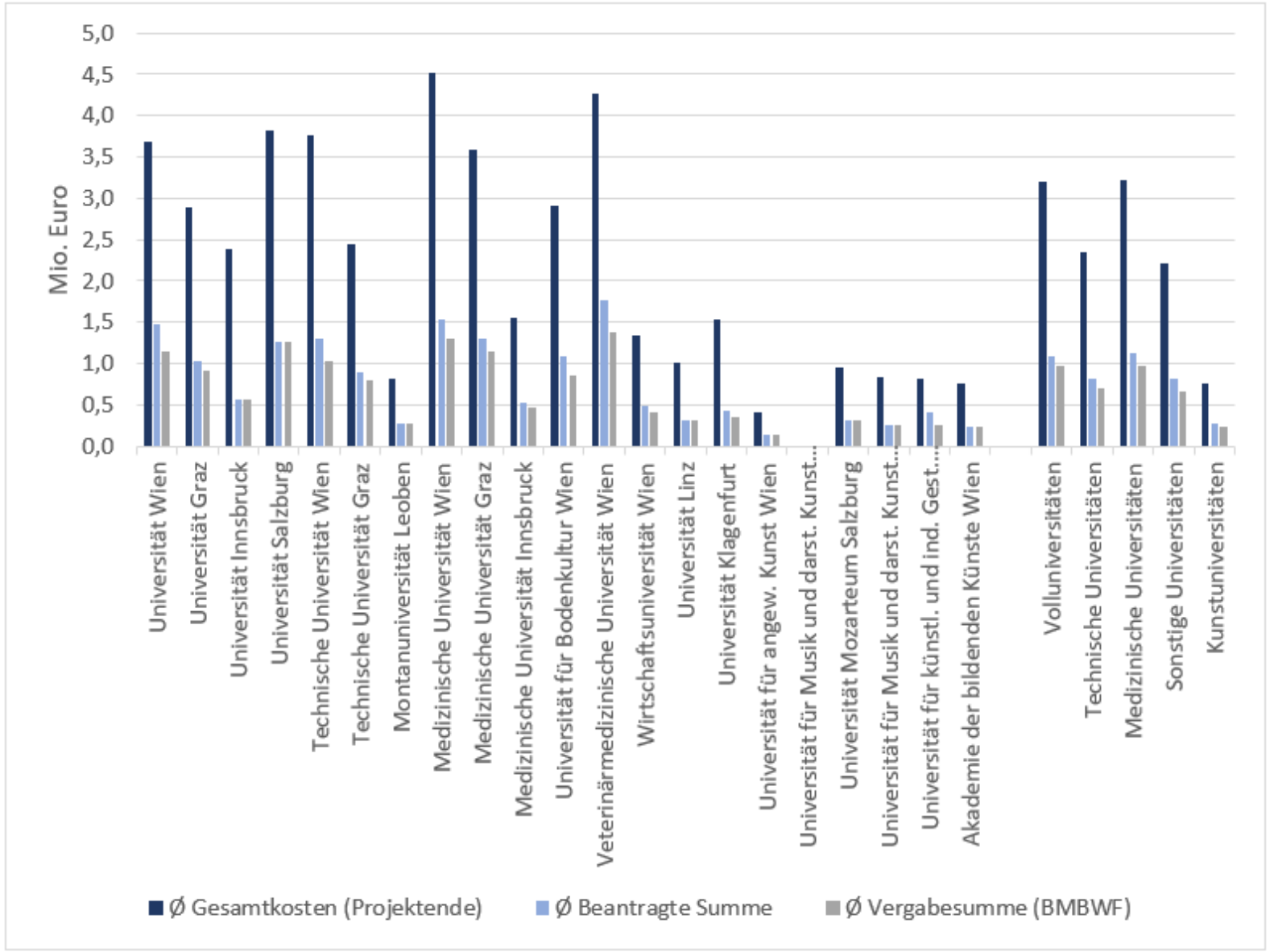

Quelle: BMBWF, eigene Berechnungen; $n=83$

Auf einem Blick:

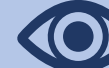

Von den 218 beantragten Projekten in der Ausschreibung der Anschubfinanzierung von Kooperationen der Universitäten in Lehre und Forschung/Entwicklung und Erschließung der Künste sowie Verwaltung im Jahr 2013 wurden insgesamt 83 Projekte durch das BMBWF finanziert. Insgesamt wurden 63 Mio. Euro vergeben, davon entfielen $8 \%$ (5,17 Mio. Euro) auf den Bereich Lehre, 68 \% (42,83 Mio. Euro) auf den Bereich Forschung/EEK und $24 \%$ (15,0 Mio. Euro) auf den Bereich Verwaltung. Im Rahmen der HRSM-Ausschreibung 2013 hat das BMBWF grundsätzlich ein Drittel der gesamten Projektkosten finanziert.

Die Volluniversitäten konnten durch ihre Größe und das breite Spektrum an verschiedenen Disziplinen und Fachgebieten die meisten Anträge stellen und dadurch mit durchschnittlich acht Projekten die meisten Bewilligungen einwerben. Die Kunstuniversitäten zeigen im Vergleich mit durchschnittlich 1,8 finanzierten 
Projekten die wenigsten Projekte pro Universitätstyp auf. Dieses Bild spiegelt sich auch in der Höhe der eingeworbenen HRSM-Mittel wider: So konnten die Volluniversitäten mit rund $43 \%$ (26,9 Mio. Euro) die meisten Finanzierungsmittel einwerben, gefolgt von den Medizinischen Universitäten mit $21 \%$ (12,95 Mio. Euro), den Sonstigen Universitäten mit 17 \% (10,92 Mio. Euro), den Technischen Universitäten mit 15 \% (9,46 Mio. Euro) und den Kunstuniversitäten mit 4 \% (2,77 Mio. Euro).

Zusammenfassend kann festgehalten werden, dass durchaus Unterschiede in den Bewilligungsquoten, den beantragten und den im Rahmen der HRSM-Ausschreibung 2013 tatsächlich vergebenen Mitteln zwischen den Bereichen Lehre, Forschung/EEK und Verwaltung sowie Universitätstypen evident sind.

Für die HRSM-Ausschreibung 2013 wurde seitens des BMBWF festgelegt, dass die Finanzierung durch das Ministerium auf ein Drittel der Projektkosten beschränkt ist. Mit $60 \%$ schätzte eine Mehrheit der befragten Projektleiterinnen und Projektleiter in der Online-Befragung diesen Finanzierungsbeitrag als zu gering ein, $28 \%$ empfanden ihn als genau richtig. Diese Einschätzung traf unabhängig vom jeweiligen inhaltlichen Schwerpunkt der Projekte zu. Tendenziell sahen Projektleiterinnen und -leiter, die Projekte im Bereich der Entwicklung und Erschließung der Künste bzw. der Verwaltung durchgeführt haben, die Begrenzung der Finanzierungsmittel etwas kritischer.

\section{Abbildung 19: Begrenzung der Finanzierung mit einem Drittel der Projektkosten}

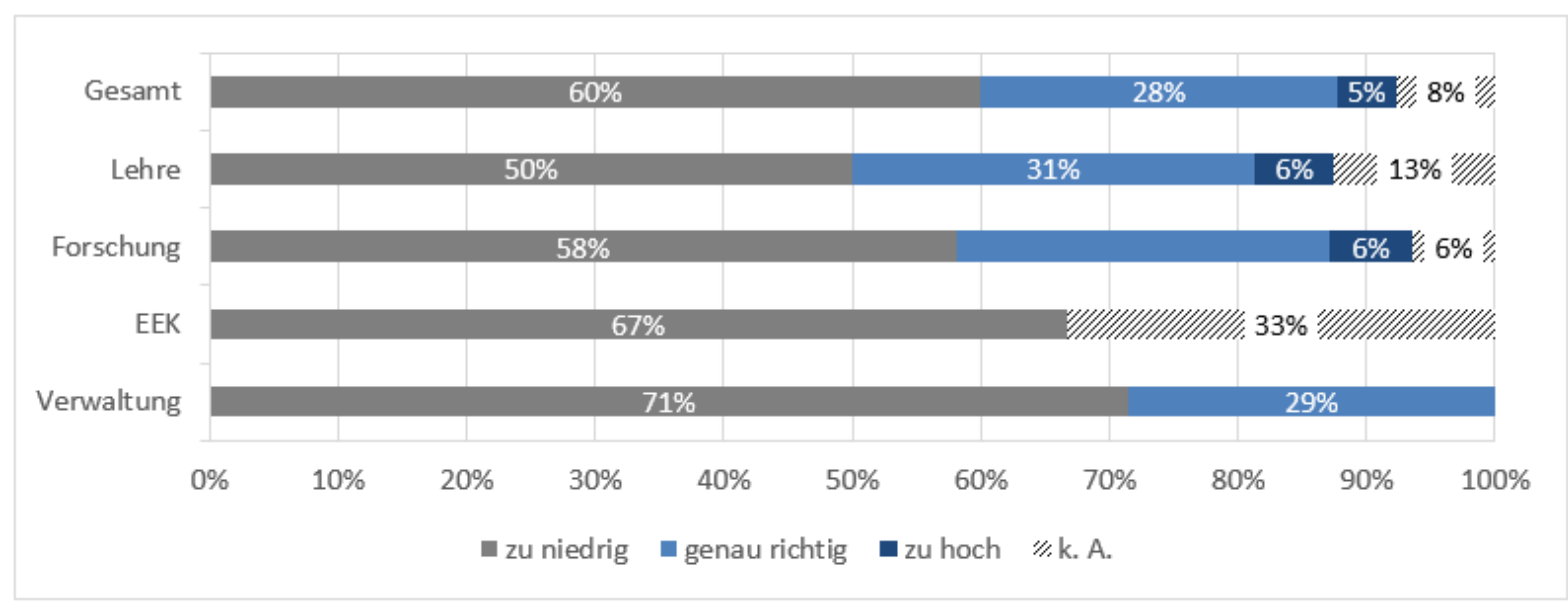

Quelle: WPZ Research Befragung der Projektleiterinnen und -leiter von finanzierten Projekten der HRSM-Ausschreibung 2013, $\mathrm{n}=65$

Ebenso wie bei den finanzierten Projekten sehen die nicht-finanzierten Projektleiterinnen und -leiter die Begrenzung der HRSM-Finanzierung von Seiten des BMBWF auf ein Drittel als zu niedrig an. Da diese Projekte die Finanzierung nicht zugesprochen bekamen, wollte knapp ein Viertel der Befragten keine Angabe zur Finanzierungsthematik machen (siehe Abbildung 20). Zwischen den verschiedenen inhaltlichen Schwerpunkten gab es kaum Unterschiede bezüglich der Wahrnehmung der Finanzierung. Für Projekte im Bereich Verwaltung liegt nur eine sehr geringe Stichprobe vor, sodass Befunde hier nicht gesondert interpretiert werden sollten. 


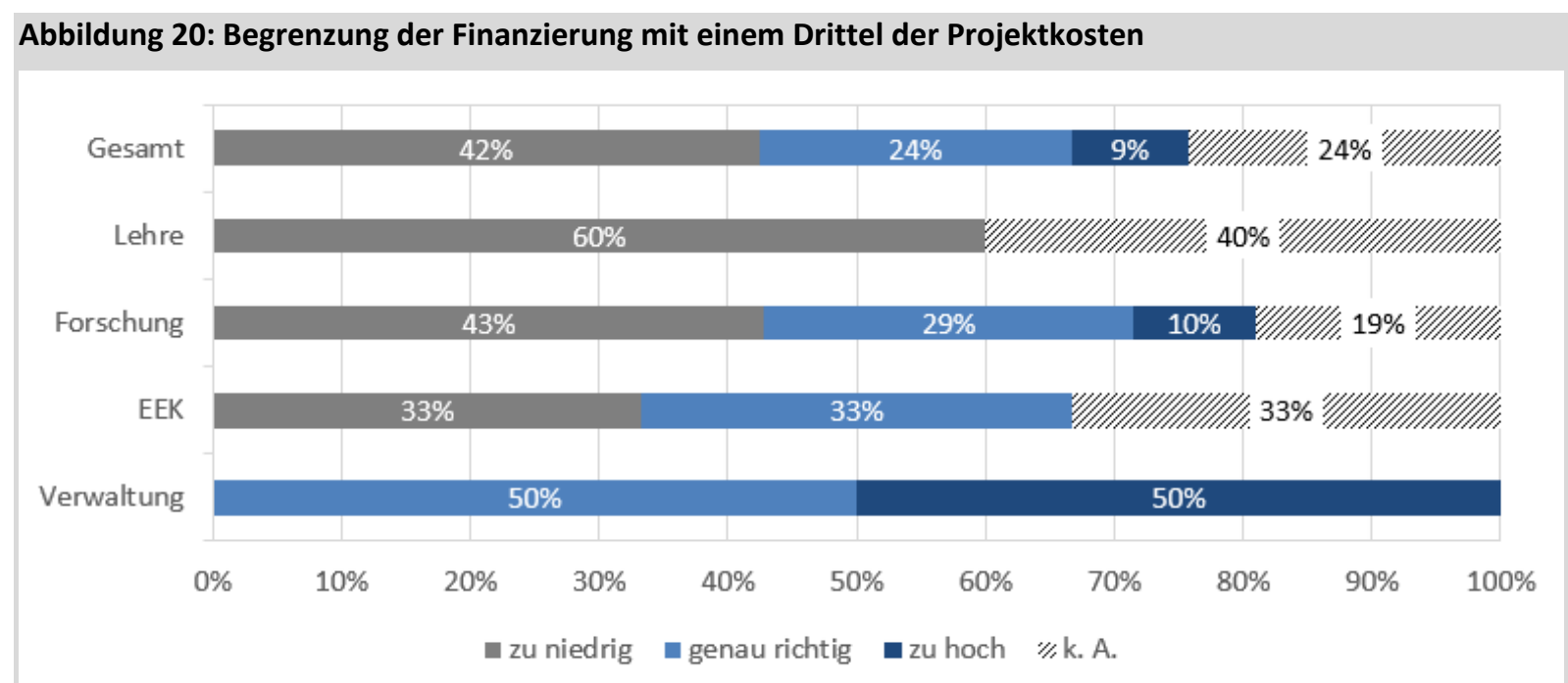

Quelle: WPZ Research Befragung der Projektleiterinnen und -leiter von nicht-finanzierten Projekten der HRSM-Ausschreibung $2013, n=33$

Dementsprechend war auch die Hälfte (51\%) der Projektleiterinnen und -leiter der Meinung, dass eine solche Begrenzung der Finanzierungsmittel seitens des BMBWF behindernd und nicht anreizfördernd wirkt. Um tatsächlich Anreize zu schaffen, wünschten die Projektleiterinnen und -leiter einen deutlich höheren Finanzierungsanteil. Einige gaben an, dass der Finanzierungsanteil zumindest 50:50 betragen sollte, andere hielten eher einen Finanzierungsanteil in der Höhe von 75 \% durch das BMBWF für anreizfördernd.

\section{Abbildung 21: Begrenzung der Finanzierung mit einem Drittel der Projektkosten}

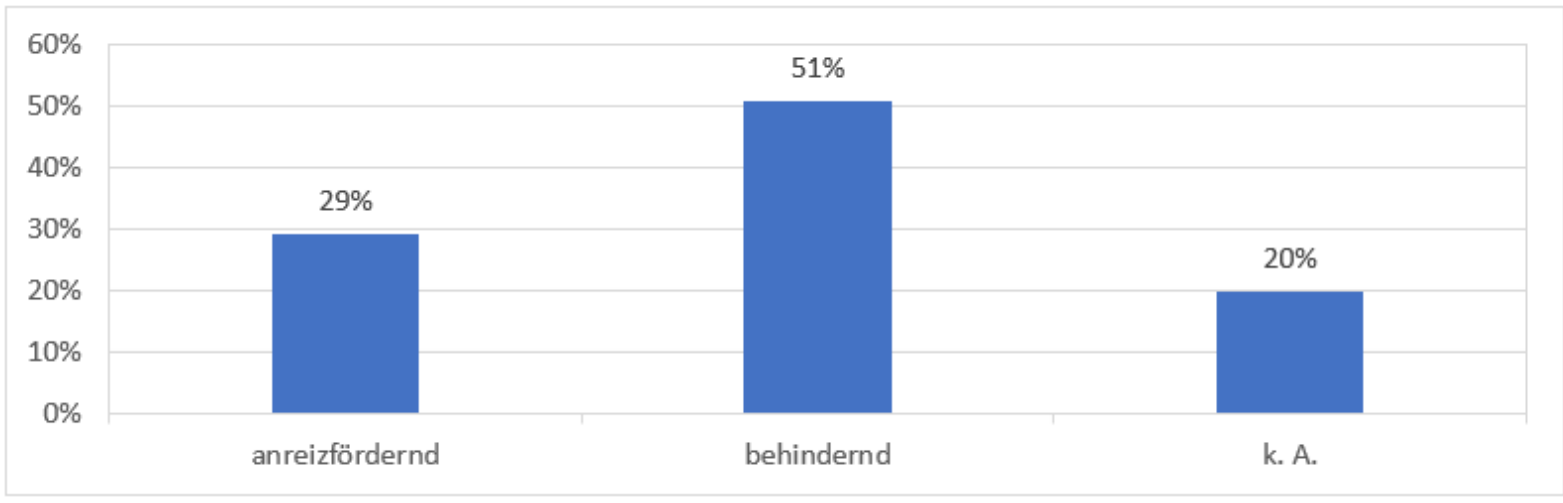

Quelle: WPZ Research Befragung der Projektleiterinnen und -leiter von finanzierten Projekten der HRSM-Ausschreibung 2013, $\mathrm{n}=65$

Neben dem Finanzierungsanteil des BMBWF bestritten die Universitäten ihre HRSM-Projekte vorwiegend aus dem Globalbudget (86\%). Mit $41 \%$ gab ein erheblicher Anteil der Projektleiterinnen und -leiter an, dass auch Drittmittel zur Finanzierung herangezogen wurden. Der Rest entfiel hauptsächlich auf Mittel der Kooperationspartnerinnen und -partner (siehe Abbildung 22). 


\section{Abbildung 22: Finanzierungsmittel}

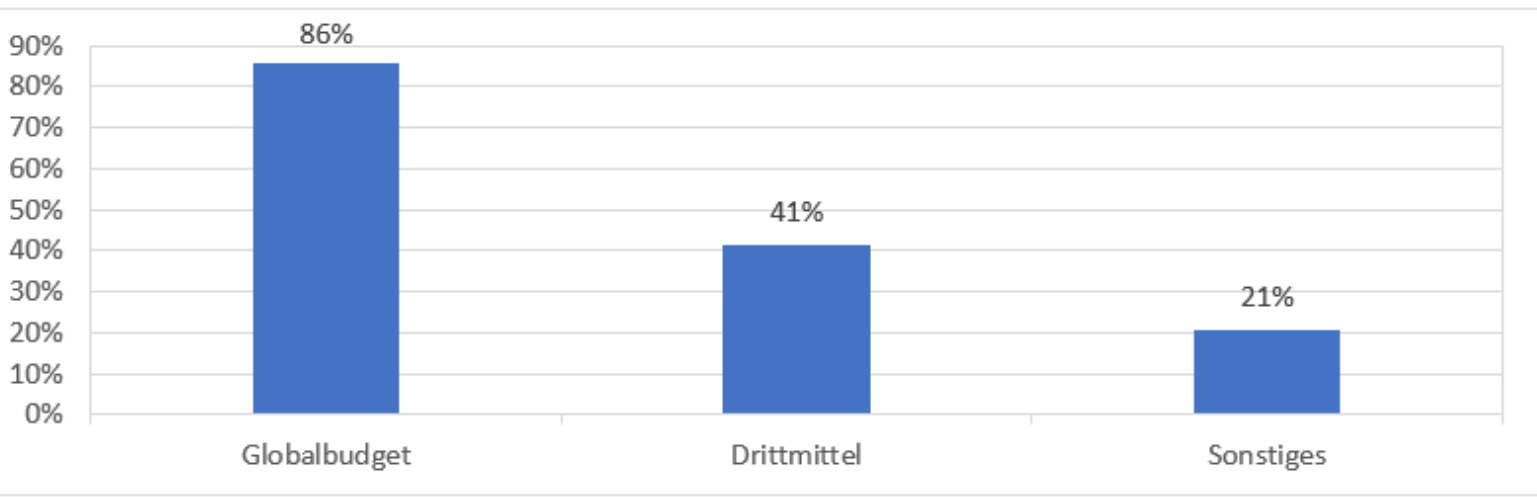

Quelle: WPZ Research Befragung der Projektleiterinnen und -leiter von finanzierten Projekten der HRSM-Ausschreibung 2013, $\mathrm{n}=63$

Im Zuge der Online-Befragung gaben $52 \%$ der Projektleiterinnen und -leiter an, dass ihr HRSM-Projekt Thema bei den Begleitgesprächen zur Leistungsvereinbarung zwischen Universität und BMBWF war. 39 \% konnten keine Auskunft darüber geben.

Den Projekt- und Finanzierungszeitraum der HRSM schätzten die Projektleiterinnen und -leiter zu $86 \%$ als genau richtig ein. Gemäß Analyse der Anträge und Endberichte (siehe oben) betrug der durchschnittliche Projekt- und Finanzierungszeitraum fünf Jahre. Die wenigen Personen (13\%), die den Zeitraum als zu kurz empfanden, würden eine Projektdauer von rund sechs Jahren, also ein Jahr länger, bevorzugen.

\section{Abbildung 23: Dauer des Projekt- und Finanzierungszeitraums}

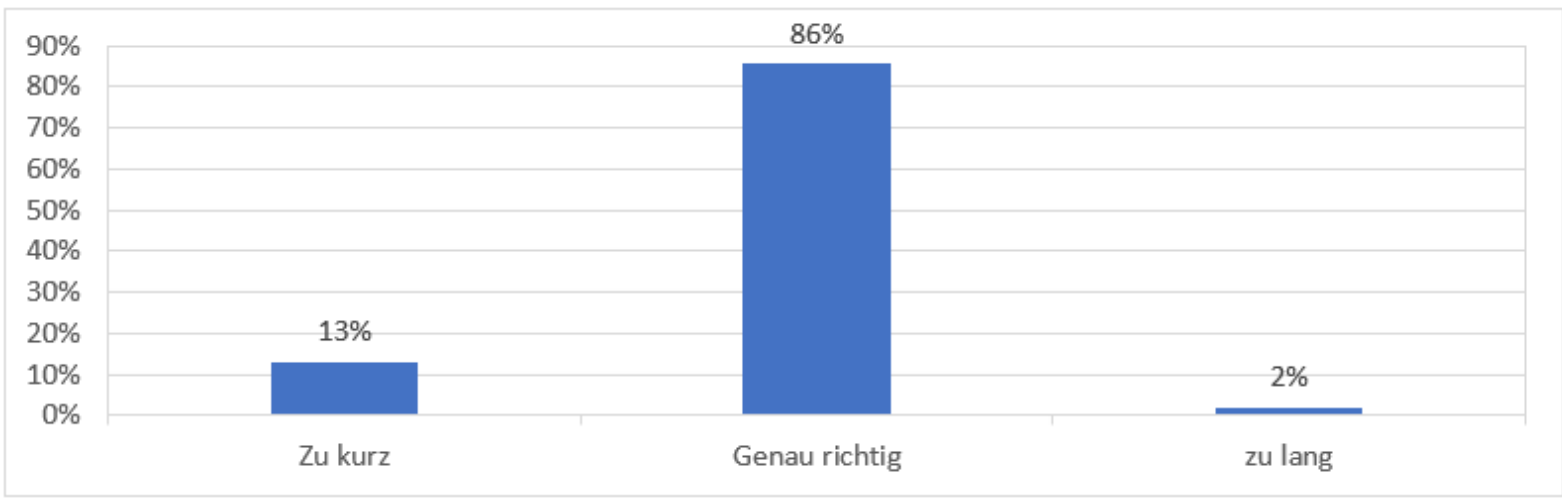

Quelle: WPZ Research Befragung der Projektleiterinnen und -leiter von finanzierten Projekten der HRSM-Ausschreibung 2013, an $=64$

Auch den administrativen Aufwand für das Reporting, also für das Verfassen der Zwischen- und Endberichte, hielten die Projektleiterinnen und -leiter für angemessen. Mit 83 \% gab die überwiegende Mehrheit an, dass der Aufwand genau richtig sei, lediglich $17 \%$ schätzten ihn als zu hoch ein. Auch auf einer inhaltlichen Ebene empfanden die Projektleiterinnen und -leiter das Reporting als sinnvoll. $66 \%$ hielten es für (eher) zweckmäßig, 24 \% für mittel und lediglich 10 \% für (eher) nicht zweckmäßig. 


\section{Abbildung 24: Zweckmäßigkeit des Reportings}

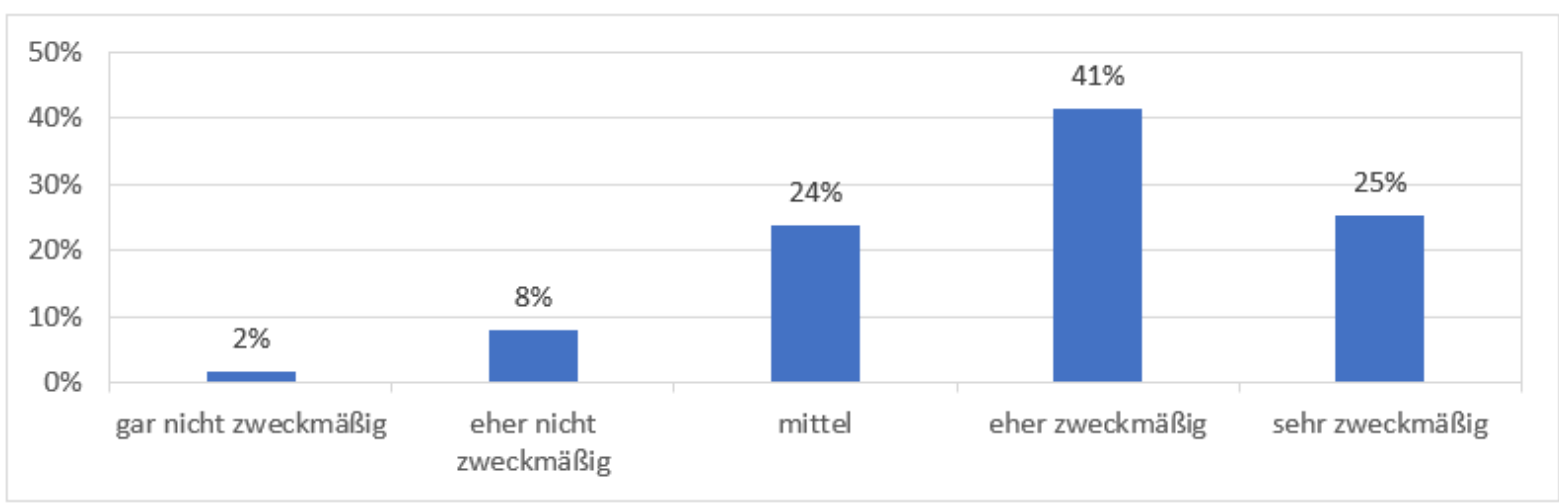

Quelle: WPZ Research Befragung der Projektleiterinnen und -leiter von finanzierten Projekten der HRSM-Ausschreibung 2013, $n=63$

Bei der Umsetzung ihrer HRSM-Projekte hatte mit $73 \%$ eine große Mehrheit der Projektleiterinnen und -leiter den Eindruck, dass sie seitens ihrer Universität (sehr) unterstützt wurden, lediglich $10 \%$ sahen eine (sehr) geringe Unterstützung für ihr Projekt.

\section{Abbildung 25: Universitätsinterne Unterstützung für das HRSM-Projekt}

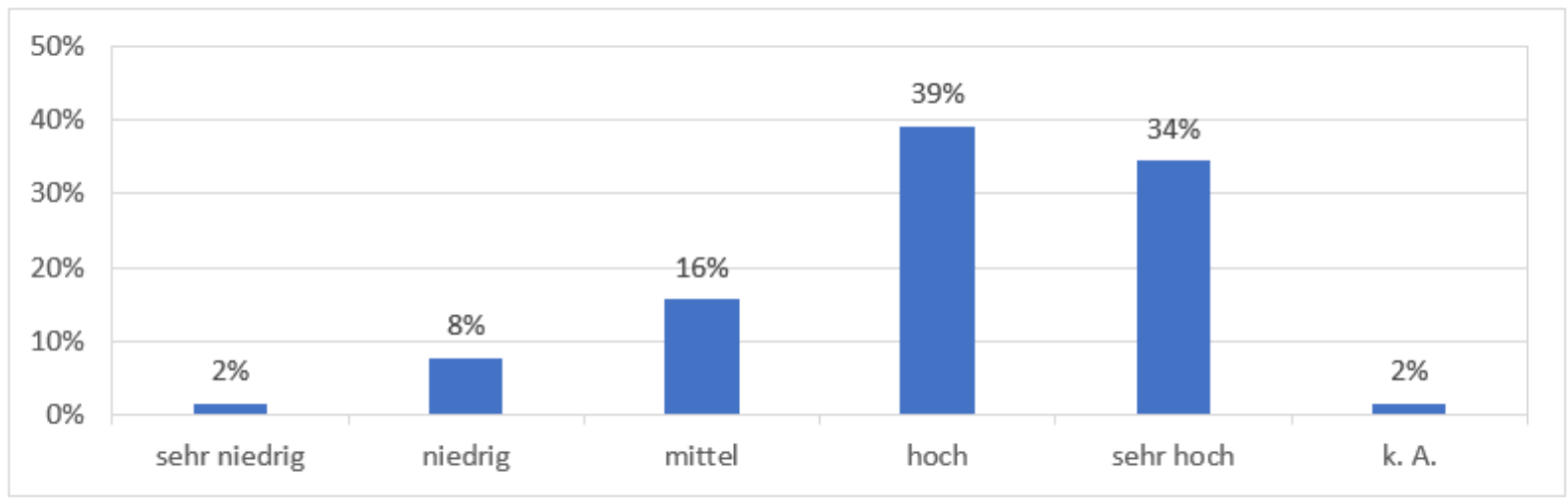

Quelle: WPZ Research Befragung der Projektleiterinnen und -leiter von finanzierten Projekten der HRSM-Ausschreibung 2013, $\mathrm{n}=64$

Die universitätsinterne Unterstützung fand sich in unterschiedlichen Akteuren. In den meisten Fällen war es das Personal in den Service- und Administrationsstellen (78 \%) oder das Rektorat (76 \%). Die übrigen Akteure folgen erst mit großem Abstand dahinter (siehe Abbildung 26). Betreffend die inhaltlichen Schwerpunkte und die Universitätstypen bestehen keine Unterschiede, was die Bereitschaft der universitätsinternen Unterstützung anbelangt. 


\section{Abbildung 26: Akteurinnen und Akteure, die das Projekt universitätsintern unterstützt haben}

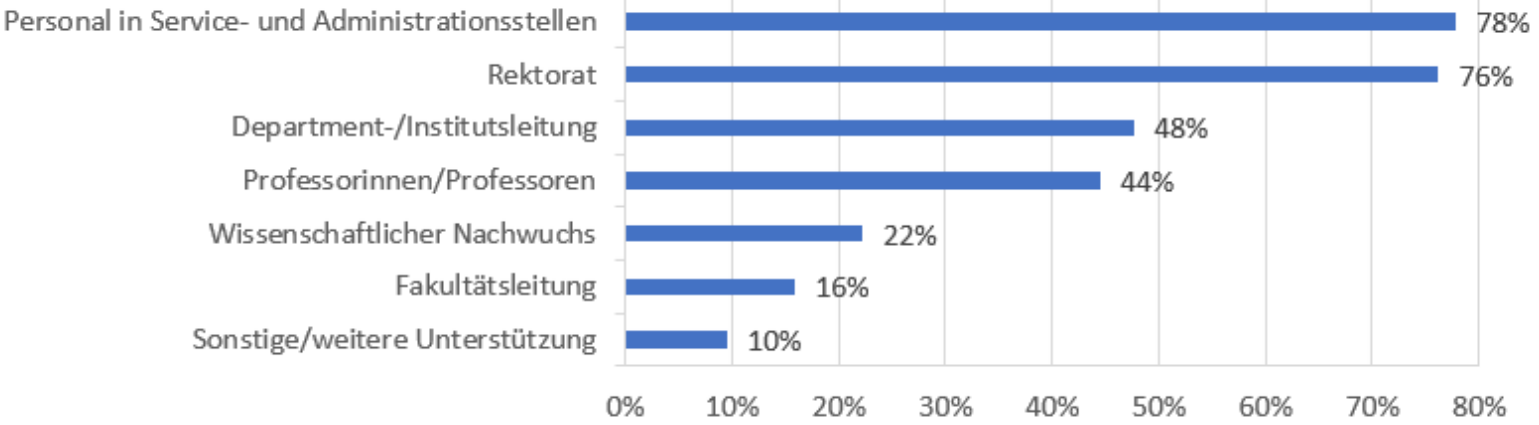

Quelle: WPZ Research Befragung der Projektleiterinnen und -leiter von finanzierten Projekten der HRSM-Ausschreibung 2013, $\mathrm{n}=64$

Bei der Befragung der Leiterinnen und Leiter von nicht-finanzierten Projekten gaben 38\% an, dass ihr Projekt nicht zu Stande gekommen ist, weil sie keine Finanzierung durch die HRSM erhalten haben. $50 \%$ haben das Projekt dennoch durchgeführt, mussten es aber redimensionieren. 13 \% konnten es sogar ohne Änderungen durchführen, weil sie andere Finanzierungsquellen erschließen konnten. Bezüglich inhaltlicher Schwerpunkte der beantragten Projekte zeigen sich hinsichtlich der Umsetzung bzw. Nicht-Umsetzung kaum Unterschiede.

\section{Abbildung 27: Umsetzung des beantragten Projekts auch ohne HRSM-Finanzierung}

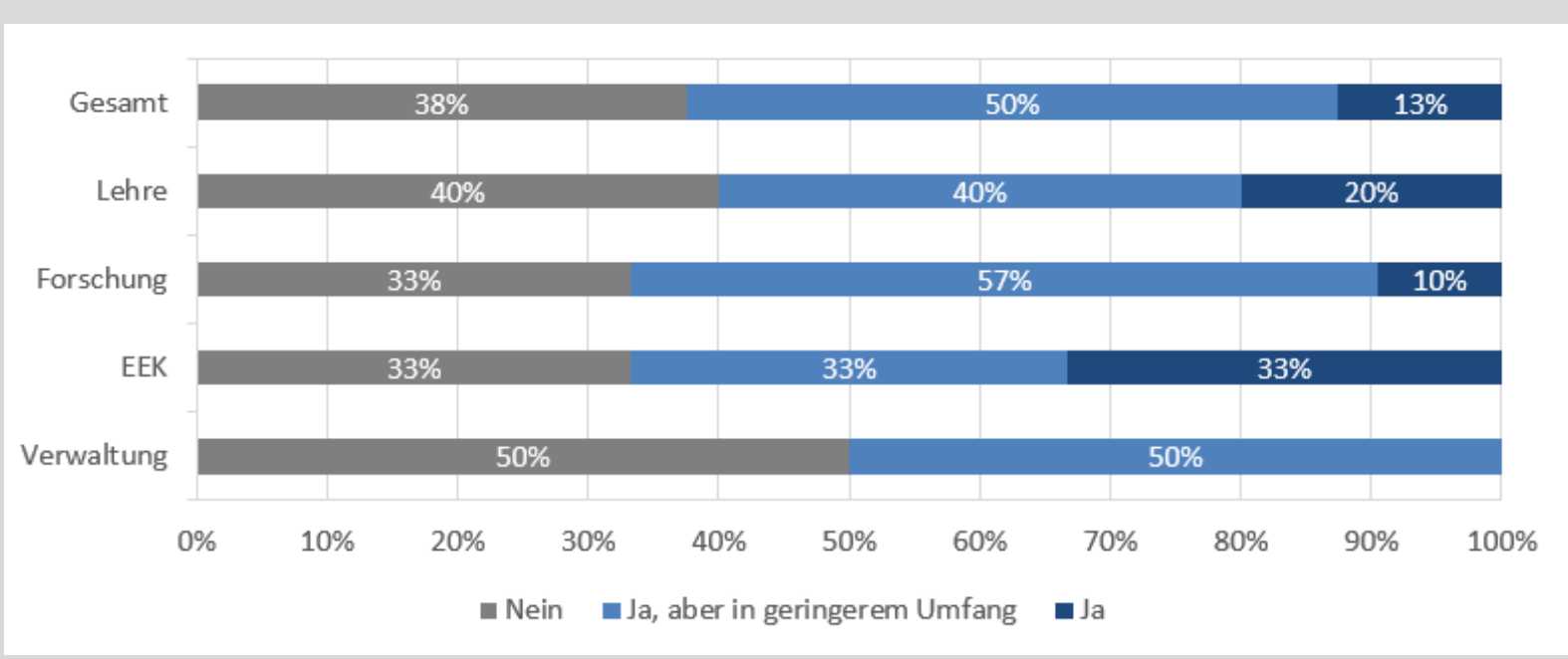

Quelle: WPZ Research Befragung der Projektleiterinnen und -leiter von nicht-finanzierten Projekten der HRSM-Ausschreibung $2013, n=32$

Die häufigste Finanzierungsquelle für das Projekt - nachdem keine Finanzierung durch die HRSM erfolgte - war das Globalbudget. Alle nicht-finanzierten Projektleiterinnen und -leiter, die an der Umfrage teilgenommen haben, gaben an, stattdessen Mittel aus dem Globalbudget bezogen zu haben. Dahinter folgen nationale Fördergeber wie FWF, WWTF oder OeNB (31 \%) und Unternehmen (25 \%) (siehe Abbildung 28). 


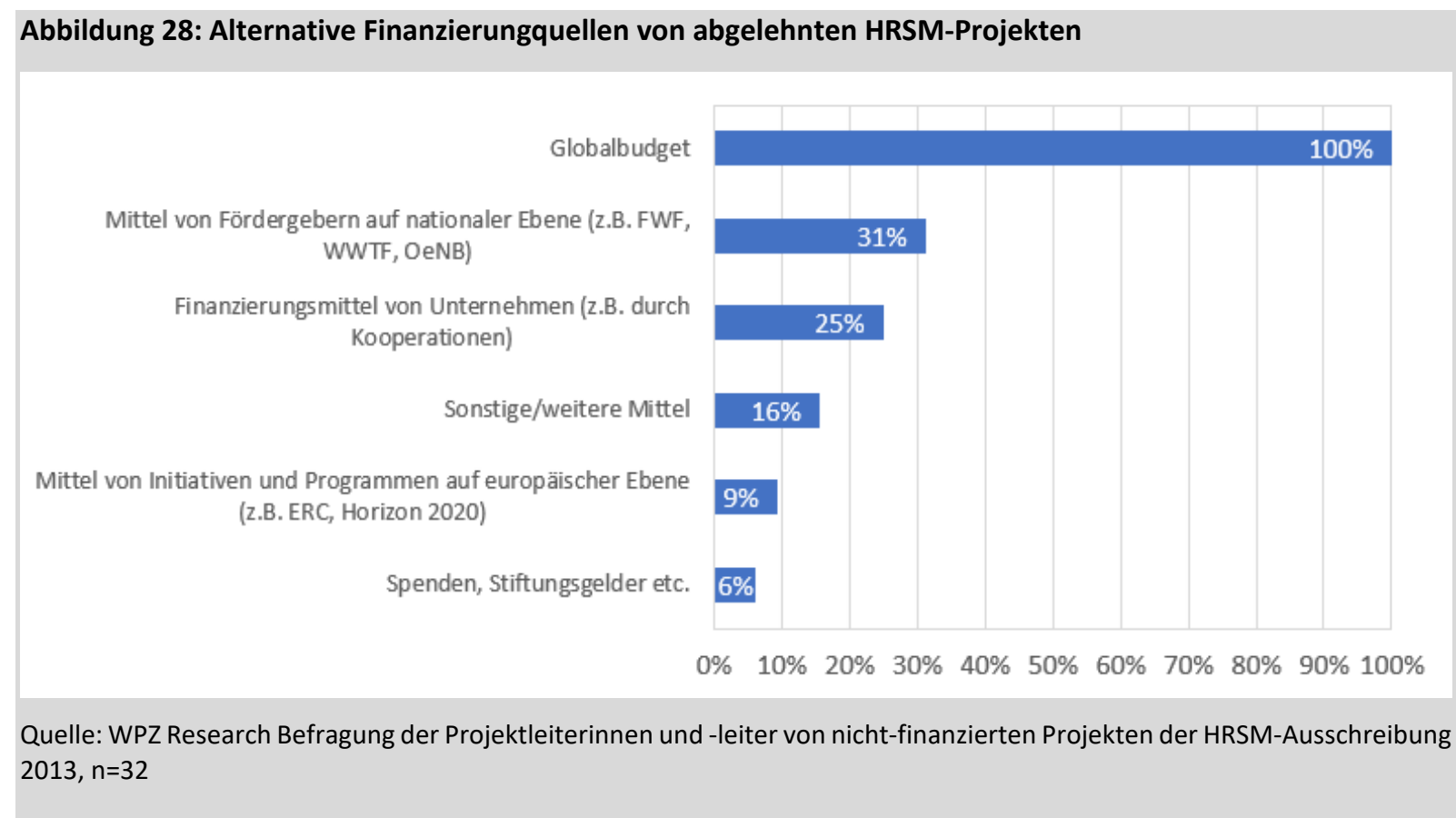

\subsubsection{Thematische Schwerpunkte}

Die Ausschreibung der Hochschulraum-Strukturmittel 2013 hatte die Förderung von nachhaltigen Kooperationen zum Ziel. Im Bereich Forschung decken die vergebenen Projekte eine große inhaltliche Bandbreite ab: Besonders gefördert wurden hier Infrastrukturkooperationsprojekte, Projekte mit Kooperationen zwischen Wissenschaft und Wirtschaft, sowie Forschungskooperationen im Bereich der "Grand Challenges" (BMBWF, 2014). Im Zuge der Antragstellung für eine Finanzierung seitens des BMBWF musste eine Selbsteinschätzung anhand verschiedener inhaltlicher Kriterien seitens der Projektverantwortlichen erfolgen. Für Projekte im Bereich Lehre und im Bereich Forschung/EEK erfolgte die Bewertung anhand von insgesamt zehn inhaltlichen Kriterien, im Bereich Verwaltung standen sechs Bewertungskriterien zur Verfügung.

Im Rahmen der Auswertung der Projektanträge zeigt sich, dass den Kriterien nachhaltige Kooperation und Etablierung gemeinsamer Strukturen die höchste Bedeutung für die inhaltliche Ausrichtung der Projekte im Bereich Lehre wie auch im Bereich Forschung/EEK zukamen. Die Bedeutung dieser beiden Kriterien wurde von $85 \%$ bzw. $75 \%$ der Projektverantwortlichen als sehr zutreffend eingeschätzt. Die Bedeutung der Kriterien Strukturbildungsbeitrag bzgl. begonnener inhaltlicher Neuausrichtungen, Management-Knowhow in/von Forschungs-Exzellenzstrukturen und Konzentrationsgrad in der Lehre wurde im Gegensatz dazu am seltensten als sehr zutreffend für die beantragten Projekte im Bereich Lehre und Forschung/EEK angegeben. 
Abbildung 29: Bedeutung der inhaltlichen Kriterien in den Bereichen Lehre und Forschung/EEK (in Prozent)

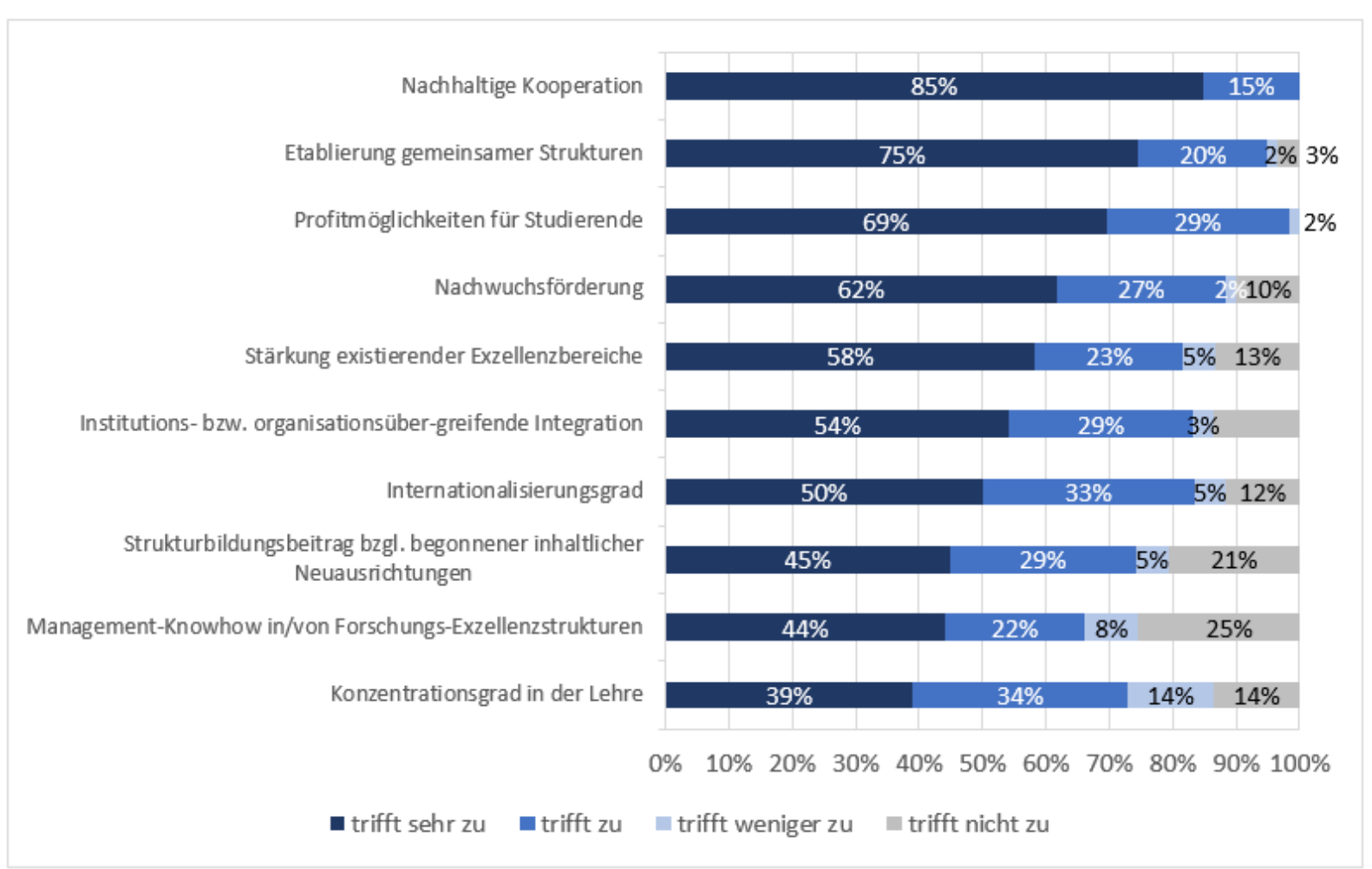

Quelle: BMBWF, eigene Berechnungen; $\mathrm{n}=60$

Bei allen 21 Verwaltungsprojekten wurden konkrete Synergieeffekte von den Projektverantwortlichen erwartet. Dahinter folgten mit 86 \% das Ziel einer nachhaltigen Effizienzsteigerung. Die übrigen inhaltlichen Kriterien folgen mit deutlichem Abstand; Innovationen/Optimierungen im IKT-Bereich wurden von den Projektverantwortlichen am seltensten als inhaltlich sehr zutreffend gesehen. Demnach ließe sich schließen, dass die Verwaltungsprojekte insbesondere auf eine verbesserte Effizienz durch Synergieeffekte abzielten.

\section{Abbildung 30: Bedeutung der inhaltlichen Kriterien im Bereich Verwaltung (in Prozent)}

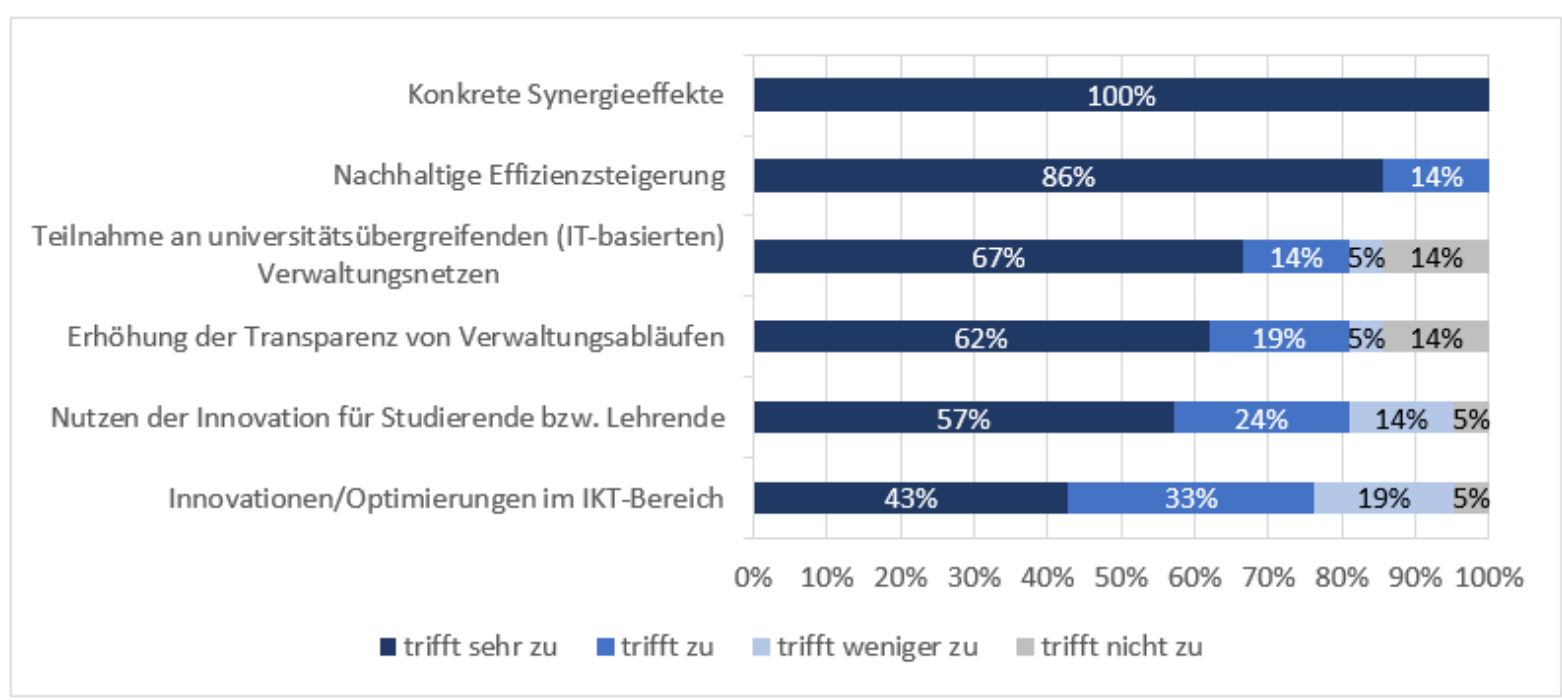

Quelle: BMBWF, eigene Berechnungen; $n=21$ 


\subsubsection{Kooperationen}

Für eine erfolgreiche Teilnahme an der HRSM-Ausschreibung 2013 war die Kooperation mit mindestens einer weiteren Einrichtung des Wissenschafts-, Hochschul-/Kunst- oder Kulturbereiches oder der Wirtschaft notwendig. $29 \%$ (24) aller Projekte hatten laut Projektantrag daher genau eine Projektpartnerin bzw. einen Projektpartner; $30 \%$ (25) aller Projekte wiesen immerhin zwei Projektpartnerinnen und -partner auf. Die restlichen $41 \%$ (34) Projekte hatten drei oder mehr Kooperationspartnerinnen und -partner (siehe Abbildung 31).

Abbildung 31: Anzahl der Kooperationspartnerinnen und -partner der finanzierten Projekte (in absoluten Zahlen)

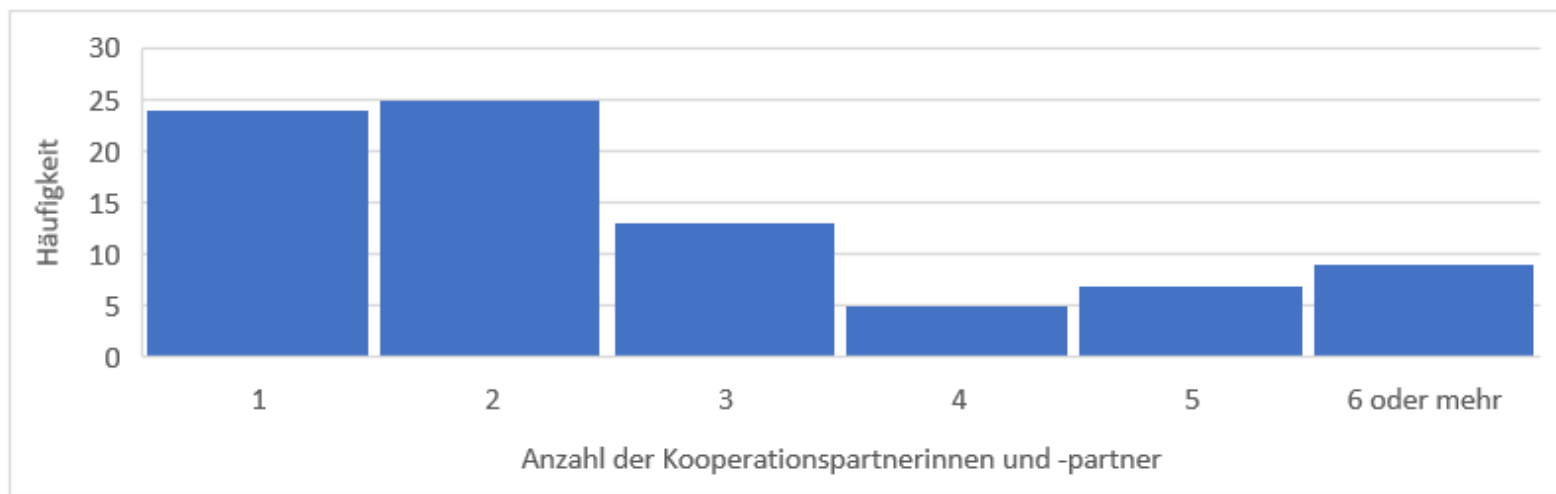

Quelle: BMBWF, eigene Berechnungen; $n=83$

Tatsächlich zeigen sich hier auch Unterschiede zwischen den einzelnen Bereichen: Hatten im Bereich Lehre etwa $35 \%$ der Projekte (insgesamt sechs Projekte) eine Kooperationspartnerin bzw. einen Kooperationspartner und 29 \% (fünf) zwei Kooperationspartnerinnen und -partner, waren es im Bereich Verwaltung lediglich 14 \% (drei) bzw. 27 \% (sechs) der Projekte. Auffällig ist, dass gerade im Bereich der Verwaltung häufiger mit mehreren Partnerinnen und Partnern kooperiert wurde. So wurden in 23 \% (fünf) der Verwaltungsprojekte Kooperationen mit mehr als sechs Partnerinnen und Partnern eingegangen; vereinzelt fanden gar Kooperationen mit 23 oder 24 Partnerinnen und Partnern statt. Dass gerade die Projekte im Bereich der Verwaltung in einem stärkeren Ausmaß auf Kooperationen setzten, lässt sich auch hier durch die Ausschreibungsschwerpunkte der HRSM 2013 erklären. So wurden insbesondere Verwaltungsprojekte gefördert, die auf die Vereinheitlichung von IT-Netzwerken der Universitäten abzielten. Laut Antragsunterlagen der Universitäten verfolgten 81 \% der Projekte im Bereich Verwaltung einen solchen Schwerpunkt.

\section{Abbildung 32: Anzahl der Kooperationspartnerinnen und -partner nach Bereich (in Prozent)}

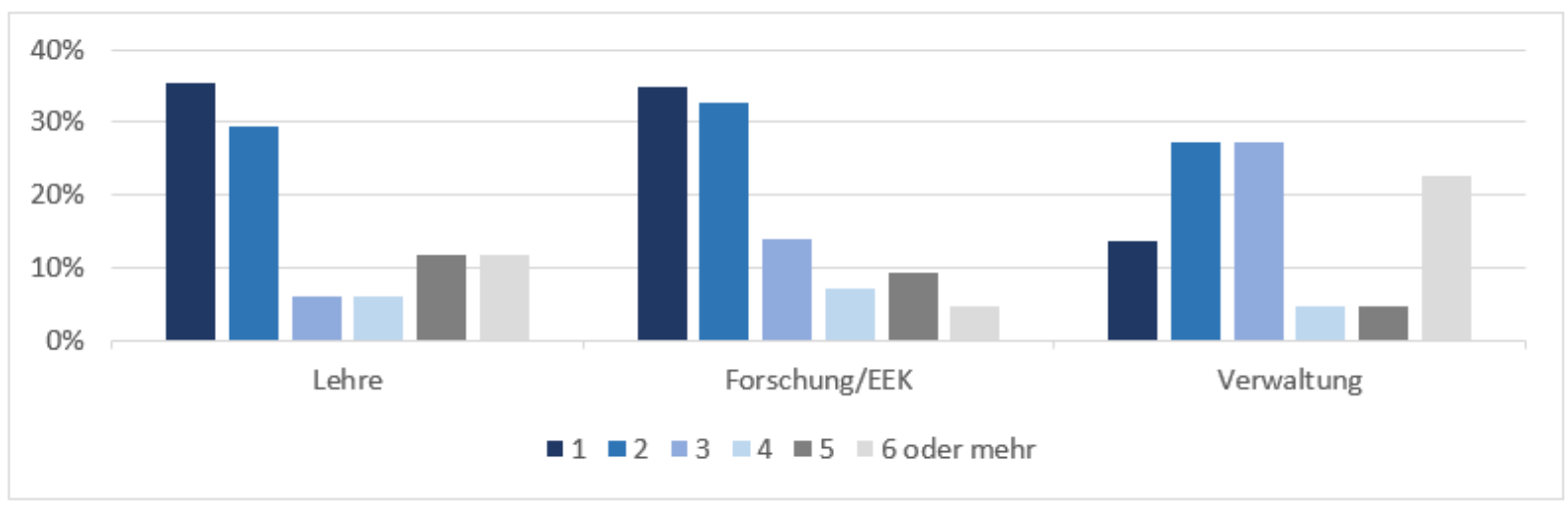

Quelle: BMBWF, eigene Berechnungen; $n=83$ 
Naturgemäß stieg mit der Anzahl der Partnerinnen und Partner auch das finanzielle Volumen der Projekte an, sodass der Hebel durch die Kooperationspartnerinnen und Kooperationspartner - was die Projektvolumina insgesamt betrifft - durchaus beträchtlich war. Hat das BMBWF im Rahmen der HRSM-Ausschreibung 2013 insgesamt 63 Mio. Euro zur Verfügung gestellt, so stellten die projektdurchführenden Universitäten und ihre Kooperationspartnerinnen und -partner insgesamt noch weitere 147 Mio. Euro bereit; d.h. das Finanzierungsvolumen der Projekte hat sich durch die zusätzlichen - von den Universitäten und Kooperationspartnerinnen und -partnern bereitgestellten - Mitteln in 2013 auf 210 Mio. Euro erhöht. Auf 1 Euro HRSM-Mittel kamen damit 2,3 Euro Ko-Finanzierung bzw. zusätzliche Mittel.

\section{Abbildung 33: Typ und Anteil der Kooperationspartnerinnen und -partner in finanzierten Projekten}

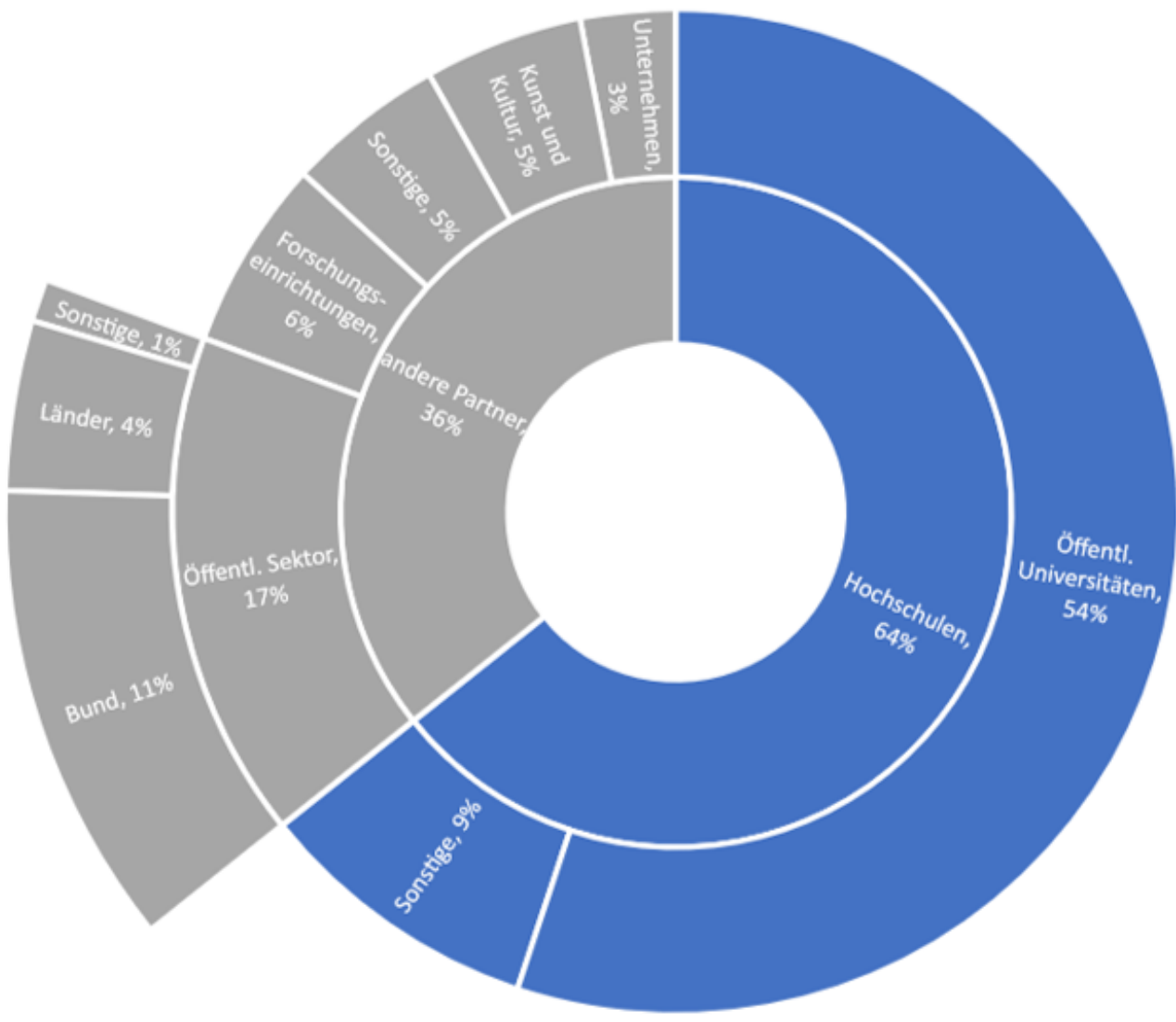

Quelle: BMBWF, eigene Berechnungen; $n=294$

Das Ziel der Anschubfinanzierung von Kooperationsprojekten im Rahmen der HRSM-Ausschreibung 2013 im Bereich Lehre, Forschung/EEK und Verwaltung war einerseits, die Zusammenarbeit zwischen den Universitäten zu fördern, und andererseits, auch die Zusammenarbeit mit anderen Einrichtungen des Wissenschafts- und Kulturbereiches sowie mit der Wirtschaft zu verbessern (BMBWF, 2014). Wie Abbildung 33 veranschaulicht, handelt es bei den HRSM 2013-finanzierten Projekten bei der deutlichen Mehrheit der Kooperationspartnerinnen und Kooperationspartner (64\%) um Hochschulen. $54 \%$ aller Kooperationspartnerinnen und Kooperationspartner sind andere öffentliche Universitäten, die restlichen $9 \%$ entfallen auf Pädagogische Hochschulen (4\%), Fachhochschulen (2\%), ausländische Hochschulen (2\%) und Privatuniversitäten (1\%). Die Verteilung der eingegangenen Kooperationen innerhalb der öffentlichen Universitäten nach Universitätstyp weist auch Unterschiede auf: 26 \% (42) der Kooperationen wurden mit Volluniversitäten eingegangen, 15 \% (24) mit Technischen Universitäten, 17 \% (27) mit Medizinischen Universitäten, 25 \% (39) mit Sonstigen Universitäten, $16 \%$ (25) mit Kunstuniversitäten und $1 \%$ (2) mit der Donauuniversität Krems.

Die übrigen $36 \%$ der gesamten Kooperationspartnerinnen und -partner entstammen nicht dem Hochschulsektor. Zur Kategorisierung dieser Partnerinnen und Partner wurde das Europäische System Volkswirtschaftlicher Gesamtrechnungen (ESVG) herangezogen. Es ist das neueste international kompatible EU-Regelwerk zur 
Rechnungslegung für eine systematische und detaillierte Beschreibung einer Volkswirtschaft und dient beispielsweise dazu, öffentliche von privaten Organisationen zu unterscheiden. Gemäß dieser Systematik entfallen $17 \%$ aller Kooperationspartnerinnen und Kooperationspartner auf den öffentlichen Sektor. Davon können etwa $11 \%$ dem Bund zugeordnet werden (z.B. Ministerien oder nachgelagerte Einheiten des Bundes wie das Bundesrechenzentrum oder das Umweltbundesamt), $4 \%$ den Ländern, und $1 \%$ entfällt auf sonstige öffentliche Einrichtungen (z.B. gesetzliche Interessensvertretungen). 6 \% sind Forschungseinrichtungen, die nicht dem öffentlichen Sektor zuzurechnen sind, wie etwa das Wiener Institut für Internationale Wirtschaftsvergleiche oder das Zentrum für Soziale Innovation. 5 \% können dem Bereich Kunst und Kultur zugeordnet werden (z.B. Naturhistorisches Museum Wien oder Oper Graz) und lediglich $3 \%$ aller Kooperationspartnerinnen und Kooperationspartner entfallen auf Unternehmen, das sind insbesondere international tätige Großunternehmen, wie z.B. Siemens, MAN, Nikon, AVL List oder die Voestalpine.

\section{Auf einem Blick:}

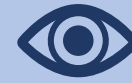

Die Projekte der Anschubfinanzierung der HRSM-Ausschreibung 2013 sind inhaltlich breit gefächert. Sie reichen von der Schaffung einheitlicher Aufnahmeverfahren für Humanmedizin und Zahnmedizin, über die Strukturförderung der Quantenforschung in Wien im Rahmen des VCQ, bis hin zum Campusmanagement an österreichischen Universitäten, welches etwa die wesentliche Verbesserung der Usability insbesondere für Studierende und Lehrende zum Ziel hatte. 39 (47\%) der insgesamt 83 finanzierten Projekte hatten den Ausbau der Forschungsinfrastruktur zum Ziel. Diese Förderung der Forschungsinfrastruktur war neben der Förderung von Kooperationsprojekten zwischen der Wissenschaft und Wirtschaft sowie Forschungskooperationen im Bereich der "Grand Challenges" ein explizites Ziel der Anschubfinanzierung der HRSM-Ausschreibung 2013 (BMBWF, 2014).

Inhaltlich erwarteten die Antragstellerinnen und Antragsteller im Bereich Lehre und Forschung/EEK vor allem eine nachhaltige Kooperation und die Etablierung gemeinsamer Strukturen. Diesen Kriterien wurde mit $85 \%$ bzw. 75 \% die höchste Bedeutung zugemessen. Bei den Verwaltungsprojekten wurden insbesondere Effizienzsteigerungen durch konkrete Synergieeffekte erwartet und folglich auch die Bewertungskriterien hierzu als inhaltlich zutreffend angesehen.

Bei der Anzahl und dem Typ der Kooperationspartnerinnen und Kooperationspartner zeigen sich Unterschiede zwischen den Bereichen Lehre und Forschung/EEK und Verwaltung. So verfügen Projekte im Bereich Lehre und Forschung/EEK meist nur über eine Kooperationspartnerin bzw. einen -partner oder zwei Kooperationspartnerinnen und -partner; Projekte im Bereich Verwaltung weisen hingegen in $23 \%$ der Fälle sogar mehr als sechs Projektpartnerinnen und -partner auf. 64 \% der Projektpartnerinnen und -partner der Universitäten waren andere Hochschulen und $36 \%$ andere Kooperationspartnerinnen und Kooperationspartner wie die öffentliche Hand, außeruniversitäre Forschungsinstitute oder Unternehmen.

Im Zuge der Online-Befragung der Projektleiterinnen und -leiter wurden auch die Kooperationen verstärkt in den Fokus genommen. Die Suche nach einer passenden Kooperationspartnerin bzw. einem passenden Kooperationspartner gelang den Projektleiterinnen und -leitern nach eigenen Angaben sehr gut. $60 \%$ bewerteten die Suche als (sehr) leicht, lediglich $7 \%$ empfanden sie als (sehr) schwierig (siehe Abbildung 34). Das mag auch daran gelegen sein, dass $90 \%$ der Projektleiterinnen und -leiter mit Partnerinnen und Partnern zusammenarbeiteten, mit denen sie bereits bei vorangegangen Projekten kooperiert hatten. Dadurch waren Kontakte bereits etabliert und erste Strukturen für eine Zusammenarbeit vorhanden. 


\section{Abbildung 34: Suche nach passenden Kooperationspartnerinnen und -partnern}

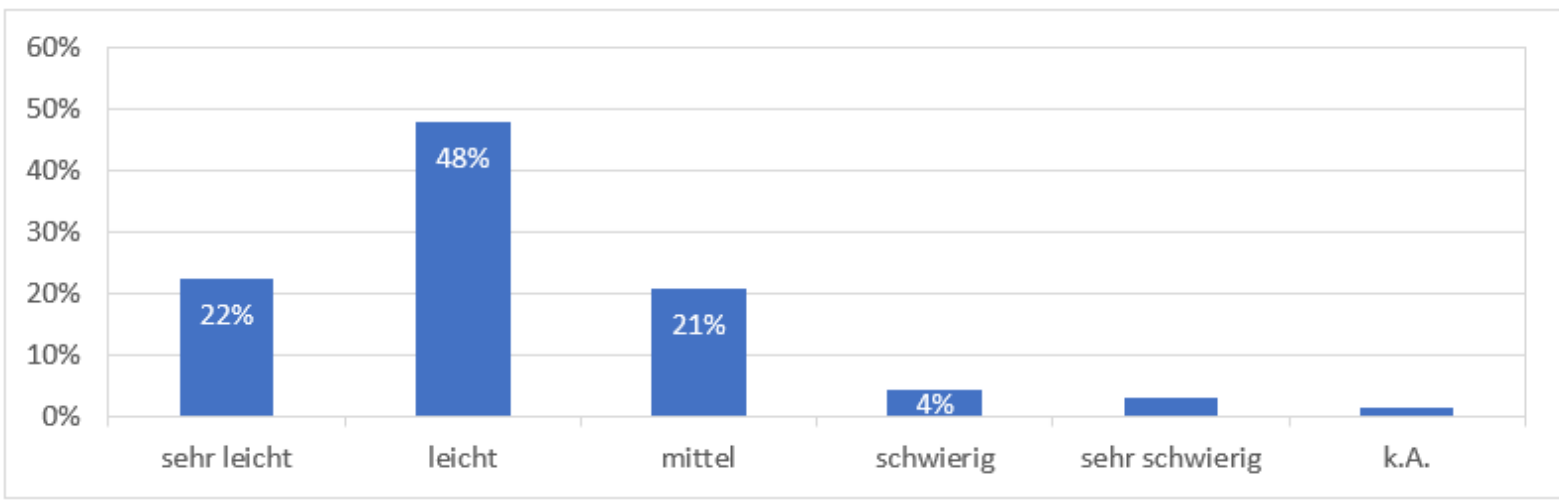

Quelle: WPZ Research Befragung der Projektleiterinnen und -leiter von finanzierten Projekten der HRSM-Ausschreibung 2013, $\mathrm{n}=67$

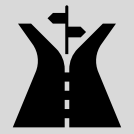

Auf der Suche nach passenden Kooperationspartnerinnen und -partnern zeigt sich: Auch wenn es den nicht-finanzierten Projektleiterinnen und -leitern im Allgemeinen durchaus leichtgefallen war, passende Kooperationspartnerinnen und -partner zu finden, hatten sie - laut Angaben in der Online-Umfrage - dennoch mehr Schwierigkeiten als die Leiterinnen und Leiter von finanzierten Projekten. $50 \%$ gaben an, dass ihnen die Suche (sehr) leichtgefallen war, bei den finanzierten Projekten waren es hingegen $70 \%$. Mit $75 \%$ wollte die überwiegende Mehrzahl dabei mit Partnerinnen und Partnern kooperieren, mit denen es bereits in der Vergangenheit eine Zusammenarbeit gab.

Im Zuge der Online-Befragung gaben die Projektleiterinnen und -leiter an, in 37 \% der Projekte mit Unternehmen kooperiert zu haben. Diese Selbsteinschätzung unterscheidet sich stark von der Analyse der Anträge und Berichte, die vom BMBWF zur Verfügung gestellt wurden. Demnach verfügen lediglich $7 \%$ der Projekte über eine Kooperation mit mindestens einem Unternehmen. Warum es hier eine so große Differenz in der Wahrnehmung gibt, lässt sich nur vage erklären. Es liegt die Vermutung nahe, dass die Projektleiterinnen und -leiter den Begriff des „Unternehmens“ in der Umfrage sehr weit gefasst und darunter auch Partnerinnen und Partner subsummiert haben, die keine Hochschulen sind (siehe hierzu Abbildung 33). So wurden womöglich öffentliche Einrichtungen und Forschungsinstitute und nicht nur privatwirtschaftliche Unternehmen in die Antwort miteinbezogen.

\section{Abbildung 35: Unternehmenskooperationen}

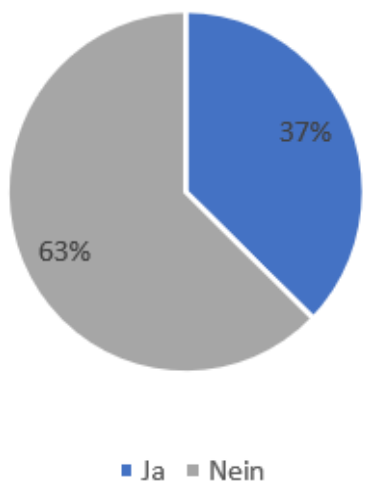

Quelle: WPZ Research Befragung der Projektleiterinnen und -leiter von finanzierten Projekten der HRSM-Ausschreibung 2013, $\mathrm{n}=66$ 
Untergliedert man die HRSM-Projekte nach thematischem Schwerpunkt, so zeigt sich, dass insbesondere bei Forschungsprojekten Unternehmenskooperationen eingegangen wurden (bei $77 \%$ aller Forschungsprojekte). Überraschenderweise wurde auch bei vielen Verwaltungsprojekten (57\%) mit Unternehmen kooperiert. Auf Lehre und EEK entfallen dagegen kaum Unternehmenskooperationen im Rahmen der HRSM-Ausschreibung 2013.

Die Projektleiterinnen und Projektleiter führten an, dass sie insbesondere deshalb nicht mit Unternehmen kooperierten, weil dies nicht den thematischen Schwerpunkten ihres Projekts entsprach. Exemplarisch werden einige Antworten aus der Online-Umfrage in der nachfolgenden Box dargestellt.

\author{
Statements der Befragten: \\ „Unternehmensbeteiligung nicht erforderlich, da es um Maßnahmen zur Organisation von strukturierten \\ Doktoratsprogrammen ging." \\ "Es kamen von der Thematik her nur sehr wenige Unternehmen in Frage. Das eine Unternehmen, das wir \\ angefragt haben, hat nicht (schnell genug) reagiert."
}

"Das Gerät findet reine Anwendung für Grundlagenforschung; Firmenkooperationen sind in diesem Zusammenhang eher ungewöhnlich."

„Das war kein Ziel der Einreichung. Ziel war eine Stärkung der Forschungsexpertise der Universitäten in einem Fachbereich."

\title{
2.2.5 Netzwerkanalyse der HRSM-Ausschreibung 2013
}

Um Kooperationen bzw. Beziehungen der Projektpartnerinnen und Kooperationspartner besser zu veranschaulichen, wurden für die HRSM-Ausschreibungen 2013 und 2016 im Rahmen der vorliegenden Evaluierung Netzwerkanalysen durchgeführt. Anhand dieser lassen sich die Anzahl der Verbindungen der verschiedenen Organisationen grafisch (wie auch numerisch) verdeutlichen. Für die HRSM-Ausschreibung 2013 wurden zusätzlich Grafiken für die drei inhaltlichen Bereiche (Lehre, Forschung/EEK sowie Verwaltung) erstellt.

Betrachtet man Abbildung 36 für die 83 Projekte der HRSM-Ausschreibung 2013, so lassen sich deutliche Cluster und zentrale Organisationen erkennen. Der Grad der Vernetztheit bzw. des Einflusses einer Organisation („Node“) wird durch die sogenannte Eigenvector Centrality (EC) gemessen. Dieser EC-Wert ist mit Werten zwischen 0 und 1 angegeben, wobei 1 auf einen besonders hohen „Einfluss" im Netzwerk hinweist (für nähere Details zur Berechnung der EC-Werte siehe Kapitel 1.3). Anhand der Breite sowie der Farbe der Verbindungen („Edges“) zwischen den Organisationen lässt sich ferner die Anzahl der Partnerschaften zwischen den zwei „Nodes" ablesen.

Die einflussreichsten Universitäten im Netzwerk, also jene Organisationen mit dem höchsten EC-Wert, sind die Universität Graz (EC-Wert=1), die Technischen Universität Graz (ebenfalls 1) und die Universität Salzburg $(0,98)$. Diese Universitäten spielen eine hohe Bedeutung durch ihre projektleitende Rolle und ihre häufige Beteiligung an Kooperationen als Projektpartnerinnen.

Den öffentlichen Universitäten kommt eine zentrale Rolle in der HRSM-Ausschreibung 2013 zu, da diese einen hohen EC-Wert, eine hohe Anzahl an Projektpartnerschaften sowie starke Verknüpfungen untereinander aufweisen. Darüber hinaus weisen sie durch ihre breite fachliche und thematische Ausrichtung ein diversifiziertes Projektportfolio auf, welches sich nicht eindeutig einem thematischen Cluster zuordnen lässt.

Ebenso lässt sich ein starkes Bündnis der Pädagogischen Hochschulen (obere Mitte) feststellen. Dieses Bündnis umfasst sämtliche Kooperationen im Bereich Lehre im Rahmen der HRSM-Ausschreibung 2013, die bereits die Umsetzung der PädagogInnenbildung Neu vorbereiteten (siehe Analyse zu Abbildung 37). Ebenso ist ein eindeutiges Cluster von öffentlichen Organisationen und Forschungseinrichtungen erkennbar (rechts oben), 
welches thematisch dem Bereich Klimawandel und Nachhaltigkeit zuordenbar ist (siehe Analyse zu Abbildung 39). Eine nähere Analyse zu diesen beiden Clustern erfolgt in den nächsten Abschnitten.

Bilateral besteht eine hohe Anzahl an Partnerschaften zwischen der Universität Graz und der Technischen Universität Graz (13 Projektpartnerschaften), gefolgt von der Medizinischen Universität Graz und der Universität Graz (10), der Medizinischen Universität Graz und der Medizinischen Universität Wien, sowie der Universität Salzburg und der Universität Graz (jeweils 9).

In diesem Kontext ist die hohe Bedeutung der geographischen Nähe für Kooperationen, beispielweise die hohe Anzahl an Partnerschaften zwischen den Grazer Universitäten hervorzuheben. Während einzelne durch die HRSM-Ausschreibung 2013 angestoßene Kooperationen bzw. Projekte in den meisten Fällen namentlich weder in den Leistungsvereinbarungen noch in den Entwicklungsplänen der einzelnen Universitäten eigens genannt werden, so sind die Kooperationen und die HRSM-finanzierten Projekte dennoch von strategischer Bedeutung und spielen in den Leistungsvereinbarungen in verschiedenen Kontexten eine Rolle. So ist beispielsweise im Kontext der hohen Anzahl von Kooperationen am Standort Graz die „Standortentwicklung und Kooperation mit anderen Hochschulen am Standort" ein wesentlicher Bestandteil der Leistungsvereinbarungen 2013-2015 der Grazer Universitäten ${ }^{4}$. Zusätzlich existieren bereits vor der HRSM-Ausschreibung 2013 mehrere institutionalisierte Kooperationen zwischen den Grazer Universitäten, wie z.B. die Forschungskooperationen BioTechMed oder NAWI Graz, welche wiederum durch im Rahmen der HRSM-Ausschreibung 2013 finanzierte Projekte unterstützt wurden.

Des Weiteren lassen sich klare thematische Bündnisse feststellen. Diesbezüglich auffällig ist beispielsweise die hohe Anzahl an Kooperationen zwischen den Medizinischen Universitäten Wien, Graz und Innsbruck. Gerade für Kooperationen im Bereich Forschung und Lehre ist es die disziplinäre Ähnlichkeit und das Nutzen von Komplementaritäten, welche die Partnerschaften begründen wie auch verfestigen. Die Stärkung der Kooperationen zwischen den Medizinischen Universitäten wird daher auch als strategischer Schwerpunkt seitens der Universitäten formuliert und ist dieser folglich auch Bestandteil der jeweiligen Leistungsvereinbarung ${ }^{5}$.

Insgesamt kann somit festgehalten werden, dass Kooperationen im Rahmen der HRSM-Ausschreibung 2013 in den Leistungsvereinbarungen bzw. Entwicklungsplänen der einzelnen Universitäten namentlich meist nicht genannt werden, allerdings die HRSM-finanzierten Projekte sich in den strategischen Zielsetzungen der Universitäten wiederfinden (die strategischen Schwerpunktsetzungen sind entsprechend breit formuliert). "Gelebt" wird die Verankerung der HRSM-Projekte augenscheinlich vor allem im Kontext der "Stärkung der Kooperationen am Standort“ und/oder bei der „Stärkung von Kooperationen zwischen Universitäten gleichen Typs", wie z.B. bei den Medizinischen Universitäten.

4 Siehe z.B. Leistungsvereinbarung 2013-2015 der Universität Graz, S. 76 ff.

5 Siehe z.B. Leistungsvereinbarung 2013-2015 der Medizinischen Universität Graz, S. 39; Leistungsvereinbarung 2013-2015 der Medizinischen Universität Wien, S. 13. 
Abbildung 36: Netzwerkanalyse für Projekte in der HRSM-Ausschreibung 2013

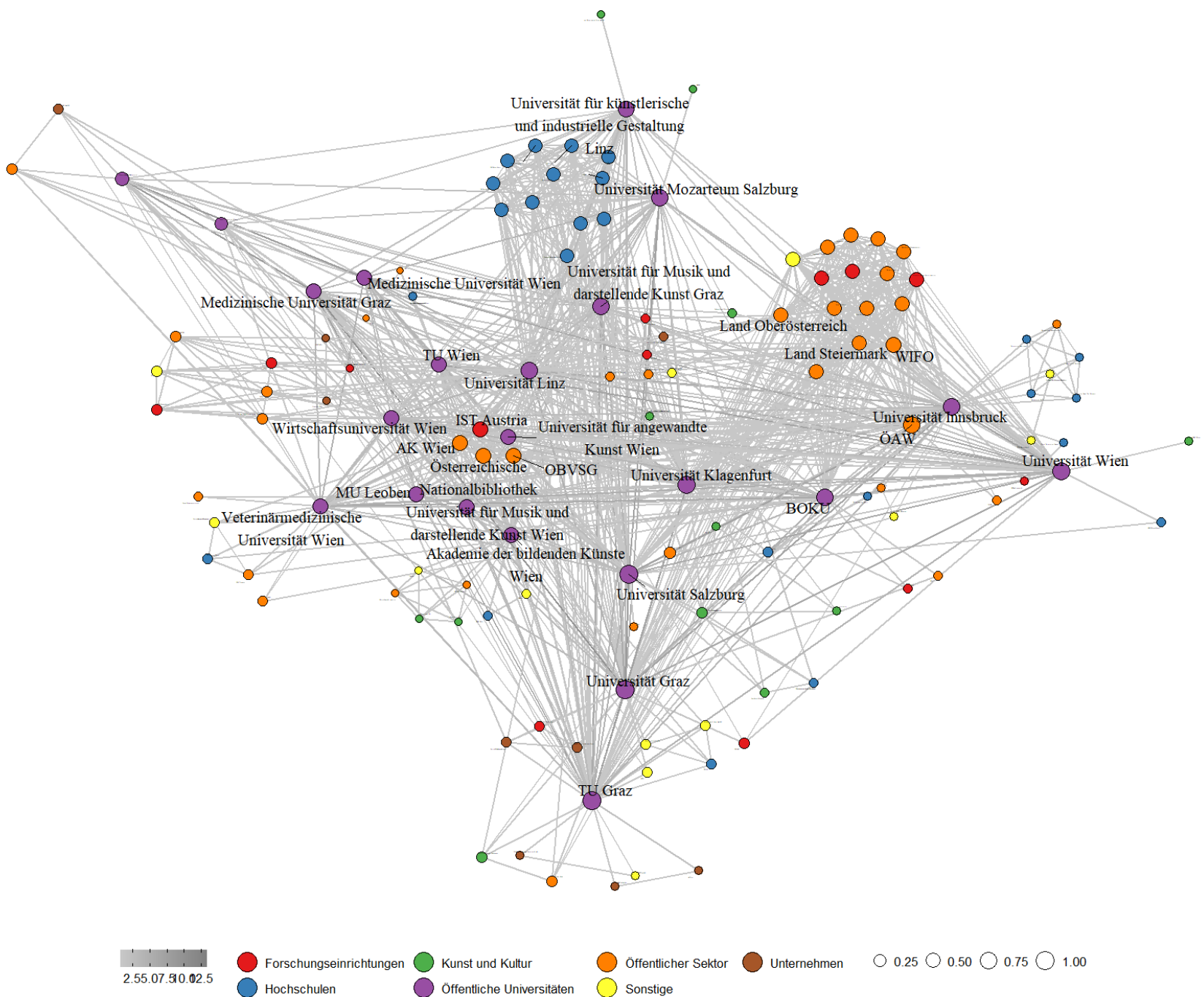

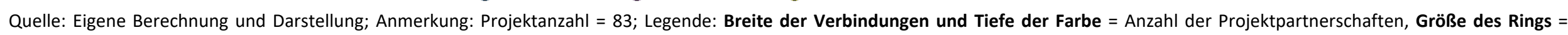
Eigenvector Centrality der Nodes, Namen der Nodes ab EC-Wert von 0,5 angezeigt. 
Ausgewählte nachhaltige Kooperationsprojekte der HRSM-Ausschreibung 2013

Ziel der HRSM-Ausschreibung 2013 war es, insbesondere Kooperationsprojekte zu finanzieren, welche der Entwicklung und Umsetzung strategisch wichtiger Vorhaben mit strukturbildender und systemischer Wirkung dienen. Neben dem, dass diese Projekte im Einklang mit den strategischen Zielen der Universitäten stehen, ist es teils auch gelungen, Kooperationen/Partnerschaften nachhaltig, d.h. über das Projektende hinaus, fortzuführen. Als einige, gerade hinsichtlich der Nachhaltigkeit gelungene Projekte, welche aus der HRSM-Ausschreibung entstammen, sind zu nennen:

MedAT: Die Kooperation der Medizinischen Universitäten im Projekt „Einheitliche Aufnahmeverfahren für Humanmedizin bzw. Zahnmedizin“ diente zur Entwicklung und Umsetzung des seit 2013 unter dem Namen MedAT durchgeführten gemeinsamen Aufnahmeverfahren für das Studium der Human- und Zahnmedizin.

Aufbau von Joint Masterprogrammen: Kooperationen im Rahmen von HRSM führten zum Aufbau von zwei Joint Master Degree Programmen. So wurde das „Erasmus Mundus Masterprogramm Astrophysics“ an der Universität Innsbruck und das „International Joint Master Degree Programme in Limnology \& Wetland Management“ an der Universität für Bodenkultur Wien aufgebaut.

Projekte zwecks Standortstärkung: Häufig ent- bzw. bestehen Kooperationen im Rahmen der HRSM-Projekte am Standort; insbesondere ist dies der Fall am Standort Graz zwischen den drei Universitäten, nämlich der Universität Graz, der Technischen Universität Graz und der Medizinischen Universität Graz. So wird diese Partnerschaft bzw. der Verbund mittels einer Reihe von Projekten wie „Zentrum für Präklinisches, Neuro-Metabolisches Imaging“, „MRT: Aufrüstung des 3 Tesla Forschungs-MRTs“, „Exzellenzzentrum für superauflösende Lichtmikroskopie“ und „OMICS Center Graz" bestärkt. All diese Projekte dienen der Stärkung der bestehenden strategischen Partnerschaften BioTechMed und NAWI Graz in den Bereichen Forschung und Lehre sowie insgesamt dem Standort Graz.

Climate Change Center Austria: Das von der Universität Innsbruck geleitete Projekt „Climate Change Center Austria“ diente dem Aufbau und der Weiterentwicklung des Klimaforschungsnetzwerks. Das CCCA ist mittlerweile Anlaufstelle für Forschung, Politik, Medien und Öffentlichkeit für alle Fragen der Klimaforschung in Österreich. Das CCCA umfasst insgesamt 22 Mitglieder aus Universitäten, Forschungseinrichtungen und öffentlichen Organisationen und hat zum Ziel, hochqualitative österreichische Klimaforschung zu betreiben, wissenschaftlichen Nachwuchs zu fördern, den Wissenstransfer zu unterstützen und Politik und Gesellschaft zu beraten.

Um die Kooperationen der HRSM-2013-finanzierten Projekte in den Bereichen „Lehre“, „Forschung/EEK“ und „Verwaltung" jeweils noch detaillierter analysieren zu können, wurden für die Bereiche separate Netzwerkanalysen durchgeführt. Die Ergebnisse werden infolge dargestellt.

\section{Netzwerkanalyse im Bereich Lehre}

Bei Kooperationen im Bereich Lehre haben die öffentlichen Universitäten eine zentrale Rolle inne - diese sind „einflussreich“ im Netzwerk (hoher EC-Wert) und weisen zahlreiche Kooperationen untereinander auf (siehe Abbildung 37). Zudem sind (Pädagogische) Hochschulen miteinander vernetzt. Ebenso sind Kunstuniversitäten in hohem Maße untereinander aktiv. Organisationen anderer Kategorien spielen im Gegensatz hierzu eine untergeordnete Rolle für Kooperationen. In dieser Hinsicht ist das Kooperationsmuster im Bereich Lehre ähnlich dem Kooperationsmuster im Rahmen der HRSM-Ausschreibung 2016 im Bereich Lehre (siehe Kapitel 3.2.5). Das Netzwerk ist charakterisiert durch ein zentrales Cluster bestehend aus öffentlichen Universitäten und Pädagogischen Hochschulen (rechte Bildhälfte). Ergänzend existieren periphere Partnerschaften (linke Bildhälfte), die keine Verbindung zu den zentralen Organisationen bzw. dem zentralen Cluster aufweisen.

Durch die sehr geringe Anzahl an Projekten und Projektpartnerinnen und Projektpartnern im Bereich Lehre ist die Repräsentation der Pädagogischen Hochschulen direkt rückführbar auf zwei Projekte mit Bezug zu Lehramtsstudien bzw. zur PädagogInnenbildung Neu. Dies ist zum einen das von der Universität Wien geleitete Projekt „Pre-/Post-Self-Assessment Lehramt“, zum anderen das von der Universität Graz geleitete Projekt „Entwicklung und Durchführung eines einheitlichen Aufnahme- und Auswahlverfahrens - PädagogInnenbildung 
Neu“. Das zentrale Cluster ist jeweils auch verankert in den strategischen Zielen der projektleitenden Universitäten.

Das Projekt „Pre-/Post-Self-Assessment Lehramt“ der Universität Wien kann strategisch auf die Bedeutung der Universität als größte Einrichtung für LehrerInnenbildung in Österreich zurückgeführt werden. Diesbezüglich ist die „qualitätsvolle, fachwissenschaftliche Ausbildung“ sowie das Ziel, die „universitäre Lehrerlnnenbildung in inhaltlicher, didaktischer und organisatorischer Hinsicht weiterzuentwickeln", strategisch in der Leistungsvereinbarung 2013-2015 festgehalten ${ }^{6}$. Das durch die HRSM finanzierte Projekt leistet damit vor allem in Bezug auf die organisatorische Weiterentwicklung des Lehramtsstudiums einen wesentlichen Beitrag.

Das Projekt „Entwicklung und Durchführung eines einheitlichen Aufnahme- und Auswahlverfahrens PädagogInnenbildung Neu“ ist ein Vorhaben, das die Umsetzung der PädagogInnenbildung Neu bereits zu einem frühen Zeitpunkt Entwicklungsverbund-übergreifend unterstützt bzw. vorbereitet und nach Projektende in Form eines Anschlussprojekts in der HRSM-Ausschreibung 2016 fortgeführt wurde. Während diese Initiative in der Leistungsvereinbarung 2013-2015 der Universität Graz zwar nicht namentlich genannt wurde, so wurden in der Leistungsvereinbarungsperiode 2013-2015 jedoch die Vorbereitungen innerhalb der Universität zur Umsetzung der PädagogInnenbildung Neu festgehalten. Demnach verfolgte die Universität Graz eine Umstrukturierung und Verbesserung der PädagogInnenbildung auf mehreren Ebenen in Partnerschaft mit anderen Institutionen, insbesondere mit den Pädagogischen Hochschulen ${ }^{7}$. Angesichts dessen ist auch die Kooperation im Rahmen des HRSM-Projekts als Unterstützung des strategischen Ziels der Universität hinsichtlich der (zukünftigen) Umsetzung der PädagogInnenbildung Neu zu sehen.

Tendenziell sind ferner thematisch ausgerichtete Kooperationsmuster zu beobachten: Medizinische Universitäten kooperieren eher unter sich, ebenso Kunstuniversitäten zu Themen, die spezifisch für ihr Studienangebot sind. So haben sich beispielsweise alle Medizinischen Universitäten zum Projekt „Einheitliche Aufnahmeverfahren für Humanmedizin bzw. Zahnmedizin“ unter der Leitung der Medizinischen Universität Wien zusammengeschlossen. Dieses Projekt diente strategisch einerseits der Verstärkung von Kooperationen unter den öffentlichen Medizinischen Universitäten ${ }^{8}$ ebenso wie dem explizit genannten strategischen Ziel, ein gemeinsames Aufnahmeverfahren zu schaffen ${ }^{9}$. Diese durch HRSM-finanzierte Kooperation führte schließlich auch zur Einführung eines österreichweiten gemeinsamen Aufnahmeverfahrens für Medizin, dem seit 2013 jährlich stattfindenden MedAT.

Die Akademie der bildenden Künste war die einzige projektleitende Kunstuniversität im Bereich Lehre. Ihre Kooperationen bilden ein in sich geschlossenes Cluster mit Organisationen im Kunst- und Kulturbereich sowie der Universität für angewandte Kunst Wien (das zweite „alleinstehende“ Cluster in der linken unteren Bildhälfte). Die Stärkung der Kooperationen mit Kunst- und Kulturinstitutionen sowie in Kooperation mit Schulen das Beratungsangebot für Studienwerberinnen und Studienwerber zu verbessern, sind strategische Ziele der Akademie der bildenden Künste Wien ${ }^{10}$. In diesem Kontext haben die HRSM-Kooperationen einen Beitrag zu den strategischen Zielen der Universität geleistet. Diese Art von Kooperationen mit anderen (Kunst-)Universitäten und Organisationen im Kunst- und Kulturbereich sind weiters ebenso Bestandteil nachfolgender Leistungsvereinbarungen ${ }^{11}$.

Im Vergleich zur HRSM-Ausschreibung 2016 im Bereich Lehre ist auch eine relativ stärkere Beteiligung von Organisationen außerhalb des Universitäts- bzw. Hochschulbereichs in der HRSM-Ausschreibung 2013 vorzufinden. Dies ist vor allem getrieben von der Technischen Universität Wien (siehe erstes „alleinstehendes“ Cluster in der linken unteren Bildhälfte) und der Technischen Universität Graz, die jeweils ein im Rahmen der HRSMAusschreibung 2013 ein Projekt leiteten und hier mit Unternehmenspartnerinnen und -partnern kooperierten so z.B. mit dem Ziel, durch gemeinsam mit der Industrie entwickelte/umgesetzte Lehrformate Studierende besser auszubilden und neue Lehr- und Lernkonzepte zu verstetigen. Die strategische Bedeutung von

\footnotetext{
Siehe Leistungsvereinbarung der Universität Wien 2013-2015, S. 72.

Siehe Leistungsvereinbarung der Universität Graz 2013-2015, S. 85.

Siehe z.B. Leistungsvereinbarung 2013-2015 Medizinische Universität Graz, S. 39.

Siehe ebenda, S. 35.

10 Siehe Leistungsvereinbarung 2013-2015 der Akademie der bildenden Künste Wien, S. 35 und S. 40.

11 Vgl. Leistungsvereinbarung 2016-2018, S. 40 bzw. Leistungsvereinbarung 2019-2021, S. 20.
} 
Kooperationen mit Unternehmen lässt sich beispielsweise auch durch die Verankerung in den Leistungsvereinbarungen erkennen ${ }^{12}$.

Verglichen mit dem Bereich Lehre der HRSM-Ausschreibung 2016 spielen internationale Partnerinnen und Partner eine relativ größere Rolle. Dies ist grafisch aus dem von der Universität Innsbruck ausgehenden peripheren Cluster (siehe untere Bildmitte) ersichtlich. Ausgehend von dem strategischen Ziel der Universität, internationale Studienprogramme mit internationalen Partneruniversitäten aufzubauen ${ }^{13}$, wurde durch die HRSM-Mittelfinanzierung 2013 das Erasmus Mundus Masterprogramm „Astrophysics“ eingerichtet, welches neben europäischen Partneruniversitäten auch die Europäische Kommission als Kooperationspartnerin umfasst. Das Joint Master Programm ist Bestandteil auch der folgenden Leistungsvereinbarungsperioden und wurde bis dato (bis 2020) in acht Durchgängen durchgeführt. ${ }^{14}$

Die Netzwerkanalyse für den Bereich Lehre zeigt insgesamt, dass das Thema „Lehramt“ im Fokus steht und darüber hinaus thematisch-orientierte Kooperationen entstanden sind bzw. bestehen - in manchen Fällen auch mit Organisationen außerhalb des tertiären Bereichs. Die Netzwerkbildung ist konsistent mit einzelnen strategischen Zielen, welche sich die Universitäten in der LV-Periode 2013-2015 gesetzt haben.

Dies wird auch durch die Analyse der Projektunterlagen unterstützt: Kooperationen im Bereich Lehre sind inhaltlich erwartungsgemäß geprägt von der Entwicklung und Umsetzung von neuen Lehr- und Lernangeboten, die je nach Projekt und Partnerkonstellation von neuen Modulen und der Einrichtung von postgradualen Studiengängen (kooperative Masterstudien, Doktoratskolleg) bis hin zu praxisnaher Lehre, beispielsweise in Kooperation mit der Industrie, reichen. Jedoch finden sich auch weitere thematische Schwerpunkte, wie z.B. die gemeinsame Unterstützung von Studienwerberinnen und -werbern (Orientierung, Self-Assessment), gemeinsame Aufnahmeverfahren, Nutzung der Möglichkeiten der Digitalisierung in der Lehre, sowie Personal- und Netzwerkaufbau. Wie bereits erwähnt wird das Instrument HRSM bzw. werden die konkreten Projekte und Partnerschaften in den Leistungsvereinbarungen der Universitäten meist nicht explizit genannt, jedoch können die durch die Projekte verfolgten Zielsetzungen mit den strategischen Zielsetzungen der Universität in der jeweiligen Leistungsvereinbarungsperiode als gut abgestimmt erachtet werden.

12 Siehe z.B. Leistungsvereinbarung 2013-2015 der Technischen Universität Wien, S. 6.

13 Vgl. Leistungsvereinbarung 2013-2015, S. 65

$14 \mathrm{Vgl}$. https://www.uibk.ac.at/astromundus/ 
Abbildung 37: Netzwerkanalyse für Projekte in der HRSM-Ausschreibung 2013 im Bereich Lehre

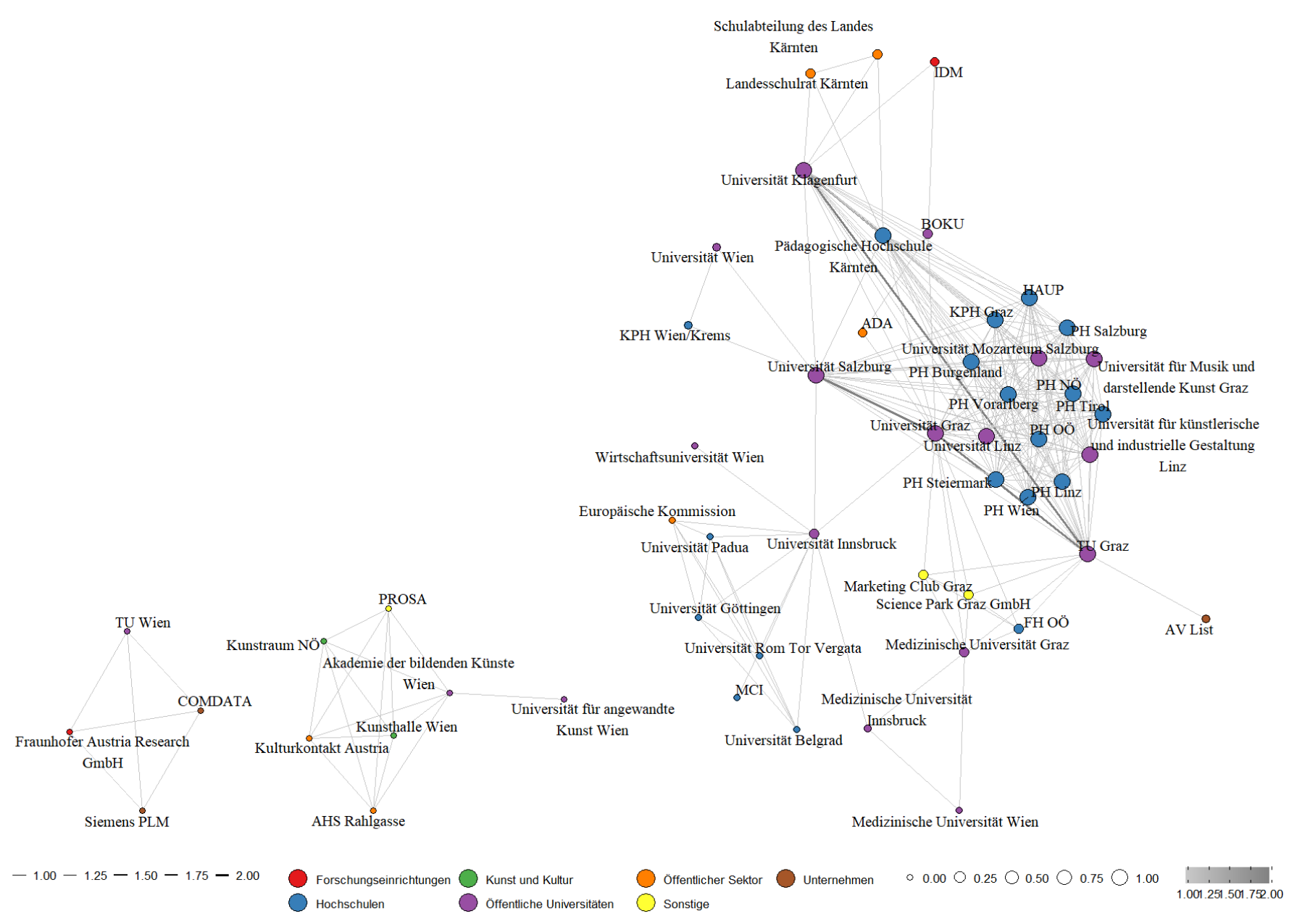

Quelle: Eigene Berechnung und Darstellung: Anmerkung: Projektanzahl = 17; Legende: Breite der Verbindungen und Tiefe der Farbe = Anzahl der Projektpartnerschaften, Größe des Rings = Eigenvector Centrality der Nodes 


\section{Netzwerkanalyse im Bereich Forschung/EEK}

Für den Kooperationsbereich Forschung/EEK zeigt sich ein diversifizierteres Bild (siehe Abbildung 38) mit weniger eindeutiger Clusterbildung. Im Bereich Forschung/EEK sind die Universitäten zentrale Akteure, Organisationen des öffentlichen Sektors sowie Forschungseinrichtungen sind auch relativ stark vertreten. Im Vergleich dazu spielen andere Organisationstypen wie Fachhochschulen, Pädagogische Hochschulen oder Unternehmen nur eine untergeordnete Rolle. Die Universität Wien spielt im Bereich Forschung/EEK die zentrale, verbindende Rolle zwischen den diversifizierten, teils sehr kleinen Clustern.

In Abbildung 37 ist vor allem ein Cluster klar ersichtlich: In der rechten Bildhälfte besteht ein klares Cluster aus privaten und öffentlichen Forschungseinrichtungen, öffentlichen Institutionen (wie dem Bundesministerium für Digitalisierung und Wirtschaftsstandort) und Universitäten, wobei letztere insbesondere durch die Wirtschaftsuniversität Wien und die Johannes Kepler Universität Linz repräsentiert werden. Dieses Cluster lässt sich disziplinär den Wirtschaftswissenschaften zuordnen und ist rückführbar auf das von der Wirtschaftsuniversität Wien geleitete Projekt „Forschungsschwerpunkt Internationale Wirtschaft" (die zuvor genannten Forschungseinrichtungen und Akteure zählen zum Projektkonsortium). Mit diesem Projekt wurde das Kompetenzzentrum „Forschungsschwerpunkt Internationale Wirtschaft - FIW“ geschaffen - mit dem Ziel, die Rahmenbedingungen zur empirischen Wirtschaftsforschung im Bereich der internationalen Wirtschaft in Österreich zu verbessern. Damit wurde eine nachhaltige Kooperation zwischen Universitäten und Forschungsinstituten geschaffen wie auch die Vernetzung und Einbindung in internationalen Projekten sichergestellt ${ }^{15}$. Langfristig soll die Kooperation mögliche weitere Zusammenarbeiten und gemeinsame Einreichungen zukünftiger Forschungsprojekte anstoßen. Entsprechend wurde die Kooperation nach Ende des HRSM-Projekts im Rahmen der Leistungsvereinbarungen 2016-2018 und 2019-2021 fortgeführt.

Bemerkenswert ist auch im Bereich Forschung die hohe Anzahl an Partnerschaften zwischen den Universitäten am Standort Graz: zum einen zwischen der Universität Graz, der Technischen Universität Graz und der Medizinischen Universität Graz, welche vor allem von vier Projekten mit Bezug zur seit 2011 institutionalisierten Forschungskooperation dieser drei Universitäten in der Initiative „BioTechMed Graz" getrieben werden; zum anderen gibt es eine enge Kooperation zwischen der Technischen Universität Graz und der Universität Graz. Ein Grund hierfür ist die bereits vor der HRSM-Ausschreibung 2013 strategische Zusammenarbeit, wie z.B. durch die seit 2004 bestehende Initiative NAWI Graz. In diesen Fällen haben die HRSM-Projekte bestehende (institutionalisierte) Partnerschaften be- bzw. verstärkt.

Insgesamt zeigt die Netzwerkanalyse im Bereich Forschung, dass öffentliche Universitäten großteils eigene Kooperationspartnerschaften und -netzwerke halten, die sich in vielen Fällen nicht überschneiden. In gewisser Hinsicht spiegelt das verstreute Netzwerk auch die thematisch-inhaltliche Diversität der HRSM-finanzierten Vorhaben wider. Davon abgesehen zeigt sich im Bereich Forschung - wie schon im Bereich Lehre -, dass die thematische Orientierung für Kooperationen durchaus von Relevanz ist. So kooperieren beispielsweise die Allgemeinen Universitäten (wie die Universitäten Wien, Linz, Innsbruck und Graz) häufig, eine klare Clusterbildung findet speziell im Bereich der Wirtschaftswissenschaften statt. Auch zeigt sich, dass die Universität Wien als große Volluniversität mit einem breit gefächerten Fächer- und Forschungsspektrum eine zentrale, verbindende Rolle zwischen den einzelnen Clustern im Netzwerk einnimmt. Die Universitäten sind darüber hinaus auch Ankerpunkt für diverse kleine, fachlich diversifizierte Netzwerke in der Peripherie; so z.B. die Universität für Bodenkultur Wien, welche eine verbindende Rolle zum peripheren Cluster bestehend aus der Veterinärmedizinischen Universität Wien sowie mehreren Forschungs- und Länderpartnern aufweist. Ähnlich wie im Bereich Lehre zeigen nur die Technischen Universitäten, in Abbildung 37 konkret die Technische Universität Graz, Partnerschaften mit Unternehmen auf.

Inhaltlich verfolgen Kooperationen im Bereich Forschung/EEK ein breit gefächertes Spektrum an Vorhaben, deren übergeordnete Zielsetzungen Profilbildung und Stärkung von bestehenden Schwerpunktbereichen sind. Die kooperativen Vorhaben erfolgen in Schwerpunktthemen, die auch in den Leistungsvereinbarungen adressiert werden und mitunter strukturbildende Maßnahmen verfolgen. Besonders häufig stehen der Netzwerkaufbau und -ausbau, gemeinsame Kommunikations- und Austauschformate, gemeinsame Forschungsvorhaben in bestehenden Schwerpunktthemen, Personalaufbau, sowie IT-, organisatorische oder Forschungsinfrastrukturinvestitionen im Fokus der Projekte.

15 Siehe Leistungsvereinbarung 2016-2018 der Wirtschaftsuniversität Wien, S. 40. 
Die Profilbildung und Stärkung von Schwerpunktthemen wurden darüber hinaus durch Projekte verfolgt, die auf die Schaffung von neuen Forschungsgruppen oder neuen Professuren abzielten. Ebenso wurden im Bereich Forschung/EEK Vorhaben in den Geistes-, Kultur- und Sozialwissenschaften finanziert. Diese Kooperationen im Kunst- und Kulturbereich wurden erwartungsgemäß von den öffentlichen Kunstuniversitäten getragen und sind im Netzwerk in der Peripherie (obere Bildhälfte) abgebildet. Häufige Kooperationspartnerinnen und Kooperationspartner sind hier Organisationen im Kunst- und Kulturbereich, vereinzelt wurden auch Partnerschaften mit weiteren Universitäten, wie z.B. mit der Universität Wien, der Universität Salzburg und der Technischen Universität Wien, eingegangen. Die Verstärkung der Kooperationen mit Kunst- und Kulturinstitutionen wie auch mit den weiteren Universitäten wird auch als wichtiges strategisches Ziel seitens der Kunstuniversitäten, wie z.B. von der Universität für Musik und darstellende Kunst Graz ${ }^{16}$, genannt. 
Abbildung 38: Netzwerkanalyse für Projekte in der HRSM-Ausschreibung 2013 im Bereich Forschung/EEK

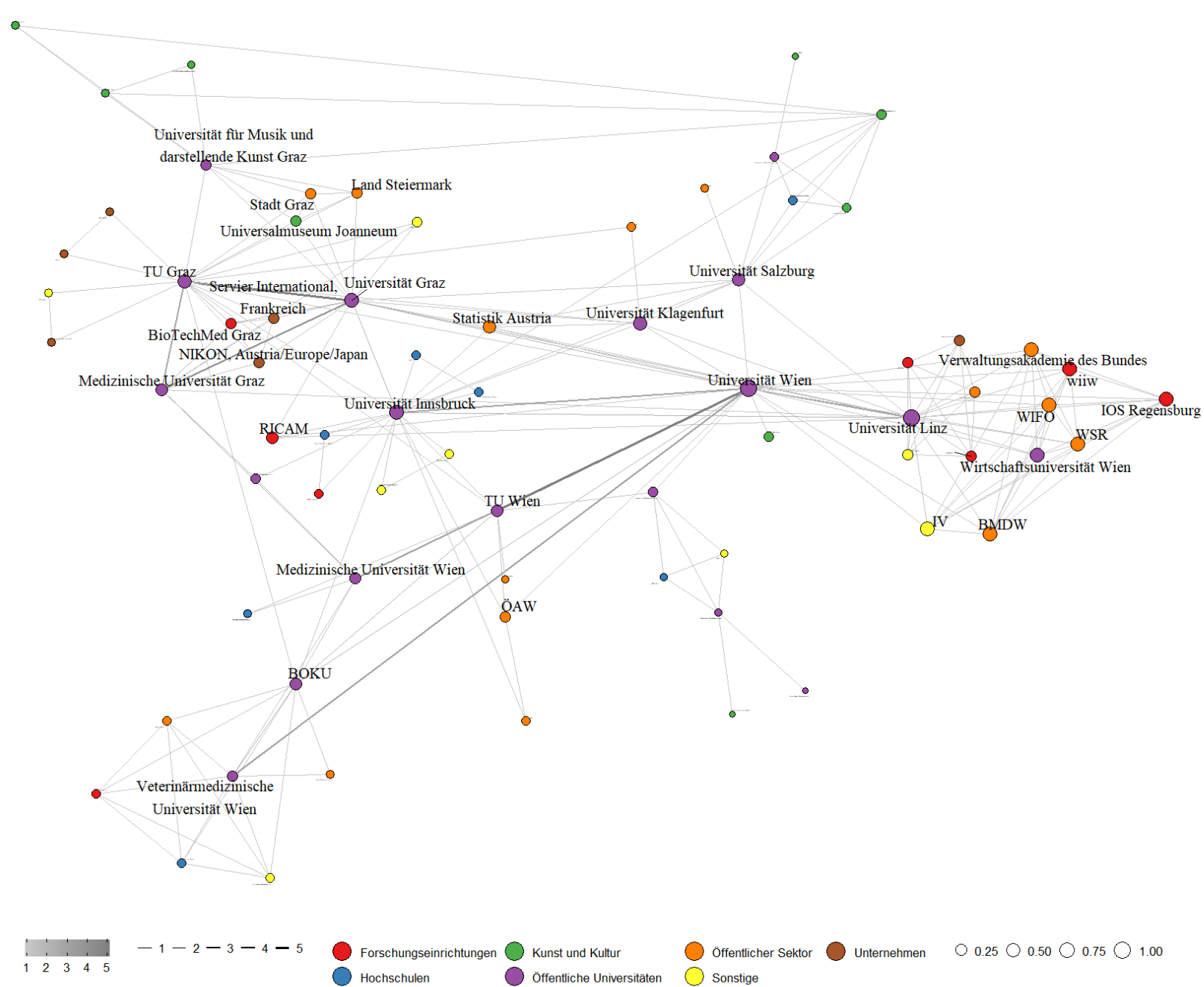

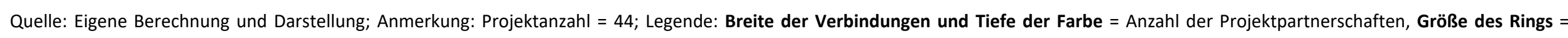
Eigenvector Centrality der Nodes, Namen der Nodes werden ab einem EC-Wert von 0,15 angezeigt. 


\section{Netzwerkanalyse im Bereich Verwaltung}

Abschließend - veranschaulicht in Abbildung 39 - zeigen sich auch im Bereich Verwaltung starke Cluster verschiedener Organisationstypen. Insgesamt ist das Netzwerk sehr dicht, periphere Verbindungen sind weniger häufig als in den anderen zuvor betrachteten Bereichen. Auch hier stehen die Universitäten im Zentrum der Netzwerke, zeigen die Universitäten ein enges Kooperationsverhalten und weisen damit auch eine verbindende Rolle zwischen den beiden anderen diversifizierten Clustern auf. Die Kooperationen im Bereich Verwaltung sind charakterisiert über eine Reihe von österreichweiten Projekten, die wesentliche administrative und organisatorische Vorhaben bei einer Mehrheit von Universitäten umsetzten - deshalb ist auch der zentrale Cluster der Universitäten in Abbildung 38 (Bildmitte) ersichtlich. Hierzu zählen die Projekte „E-Infrastructures Austria: Aufbau und Weiterentwicklung einer Repositorieninfrastruktur“, „Entwicklung und Umsetzung eines Beschaffungsprozesses für österreichische Universitätsbibliotheken“, „Einführung eines Computer Aided Facility Management (CAFM)“, "Climate Change Center Austria" und "Campusmanagement an österreichischen Universitäten“.

Zwei weitere diversifizierte Cluster sind ebenso erkennbar: Ein diversifizierteres Cluster hat sich zwischen den Universitäten, öffentlichen Institutionen und Forschungseinrichtungen gebildet (siehe rechte Bildhälfte). Dieses ist insbesondere wegen der Kooperation von fachlich unterschiedlichen Universitäten mit öffentlichen Dienstleistern interessant. Bei genauerer Betrachtung ist dieses Cluster zentral im Projekt „E-Infrastructures Austria: Aufbau und Weiterentwicklung einer Repositorien-Infrastruktur" unter der Leitung der Universität Wien verankert. Mit insgesamt 24 Kooperationspartnerinnen und -partnern stellt dieses Projekt das größte im Bereich Verwaltung im Rahmen der HRSM-Ausschreibung 2013 dar. Ziel des „E-Infrastructures Austria“-Projekts war es, nicht nur Infrastruktur in Form von Repositorien aufzubauen, sondern auch ein Wissensnetzwerk zu etablieren, schließlich auch um Folgekooperationen zu ermöglichen ${ }^{17}$. Entsprechend ist dieses Vorhaben auch strategisch in der Leistungsvereinbarung der Universität Wien ${ }^{18}$ im Themenfeld „Kooperation im Bibliotheksbereich mit dem Ziel zur Bereitstellung von Repositorien für die Forschung" festgehalten. Die Nachhaltigkeit des Vorhabens bzw. der Kooperation nach Projektende wurde durch die Verankerung in der darauffolgenden Leistungsvereinbarung gesichert. Demnach verfolgt die Universität Wien die langfristige Weiterentwicklung von Konzepten und Umsetzungen der im HRSM-Projekt aufgebauten Repositorien-Infrastruktur ${ }^{19}$.

Ein zweites dichtes Cluster besteht aus öffentlichen Organisationen und Forschungseinrichtungen (rechte Bildhälfte). Hier stellt sich die Universität Klagenfurt als wichtiges Bindeglied zu den anderen Clustern dar. Dieses Cluster lässt sich vor allem auf das Projekt "Climate Change Center Austria“ unter der Leitung der Universität Innsbruck zurückführen. Das Projektkonsortium, welches sämtliche Forschungseinrichtungen und öffentliche Organisationen mit Bezug zur Klimaforschung umfasst, hatte zum Ziel, eine Koordinationseinrichtung zur Förderung der Klimaforschung in Österreich aufzubauen. Die Universität Innsbruck spielte dabei eine zentrale Rolle, indem sie zahlreiche administrative und wissenschaftliche Aufgaben für die universitätsübergreifende Forschungsgemeinschaft übernahm. Die Aktivitäten waren auch wesentlicher Bestandteil der strategischen Ziele der Universität Innsbruck zu damaliger Zeit in Bezug auf Kooperationen ${ }^{20}$. Die Weiterführung der aufgebauten Geschäftsstelle, des Servicezentrums und des Datenzentrums sowie die Weiterentwicklung des Kooperationsnetzwerks sind außerdem in den darauffolgenden Leistungsvereinbarungen der Universität Innsbruck verankert ${ }^{21}$. Tatsächlich unterstreicht die hohe Sichtbarkeit des Climate Change Center Austria, welches aktuell u.a. einen wesentlichen Beitrag zur österreichischen Klima- und Energiestrategie leistet, die Rolle der HRSMFinanzierung für strategisch langfristige, über Universitätsgrenzen hinausgehende Vorhaben wie den Aufbau von systemwirksamen Netzwerken.

Kooperationen im Bereich Verwaltung zielten in einigen Fällen auf den Netzwerkaufbau für eine langfristige Zusammenarbeit (wie z.B. im Fall des Climate Change Center Austria) ab, andere Projekte hatten durch die Kooperationen entstandene Synergien im Fokus, so z.B. bei Beschaffungsprozessen, im Auf- und Ausbau von gemeinsamer Infrastruktur (IT-Systemen) und in der Vereinheitlichung von administrativen und organisatorischen Prozessen. Im Bereich Verwaltung ist daher der Projekterfolg nicht zwingend an der Langlebigkeit von

17 Vgl. https://e-infrastructures.univie.ac.at/das-projekt/projektziele/

18 Siehe Leistungsvereinbarung der Universität Wien 2013-2015, S. 68.

19 Vgl. Leistungsvereinbarung der Universität Wien 2016-2018, S. 80.

Siehe Leistungsvereinbarung der Universität Innsbruck 2013-2015, S. 75

21 Siehe Leistungsvereinbarung der Universität Innsbruck 2015-2018, S. 72 und Leistungsvereinbarung der Universität Innsbruck 2019-2021, S. 21 
Kooperationen festzumachen, vielmehr geht es oftmals um die Nutzung von Synergien und die damit einhergehende Kosteneffizienz. Dabei zielen Kooperationen im Bereich Verwaltung auf systemisch wirksame Vorhaben ab. Als erfolgreiche Beispiele können die beiden oben genannten Projekte, die „RepositorienInfrastruktur" wie auch das "Climate Change Center Austria“ genannt werden. Des Weiteren sind auch die Einführung von Umweltmanagementsystemen (Projekt „Umweltmanagementsysteme an Universitäten“ unter der Leitung der Universität Klagenfurt) und die gemeinsame Einführung eines Campusmanagementsystems (unter der Leitung der Technischen Universität Graz) als Good Practices für breit wirksame Vorhaben zu nennen.

\section{Abbildung 39: Netzwerkanalyse für Projekte in der HRSM-Ausschreibung 2013 im Bereich Verwaltung}

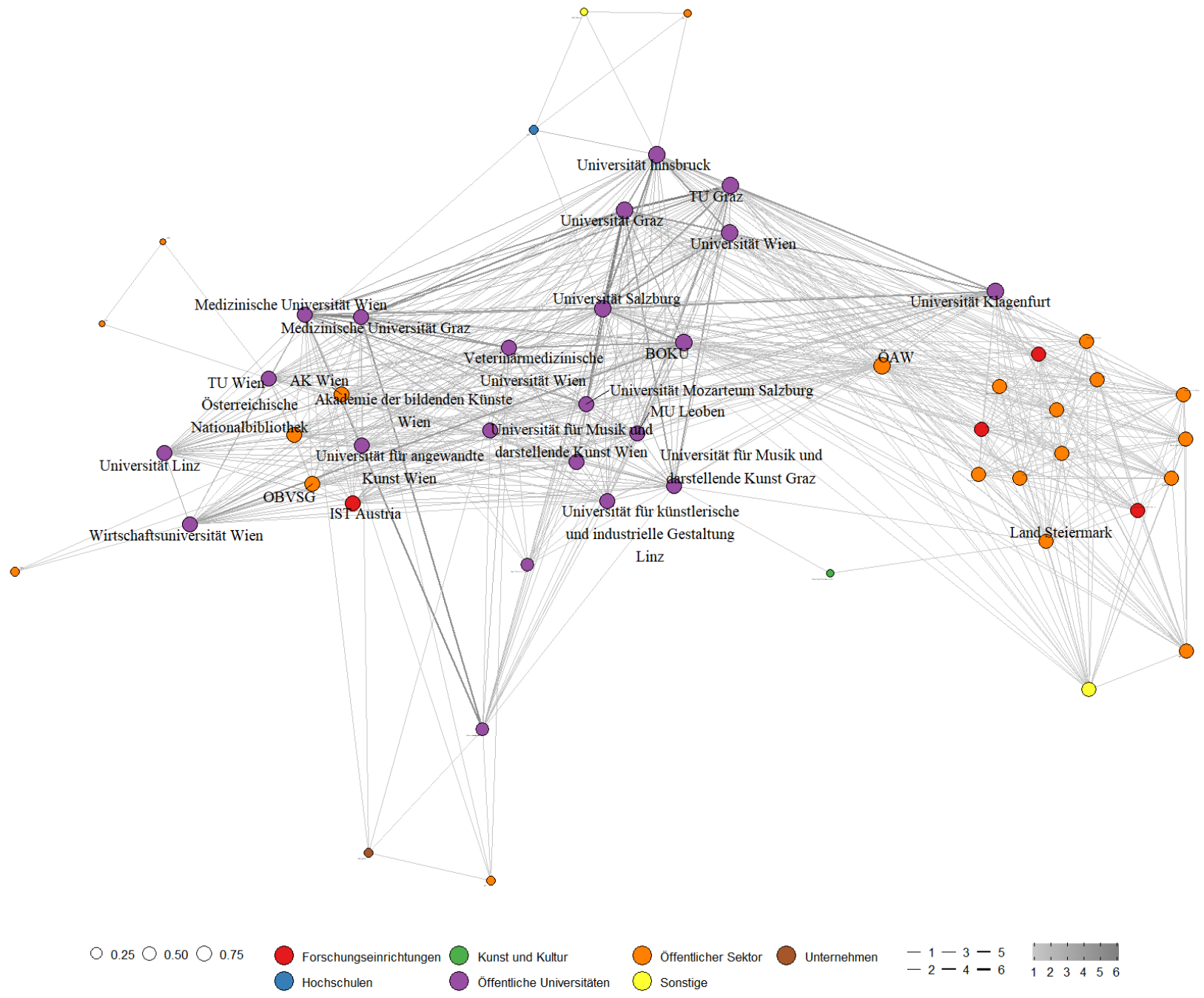

Quelle: Eigene Berechnung und Darstellung; Anmerkung: Projektanzahl = 22; Legende: Breite der Verbindungen und Tiefe der Farbe = Anzahl der Projektpartnerschaften, Größe des Rings = Eigenvector Centrality der Nodes; Namen der Nodes werden ab einem EC-Wert von 0,6 angezeigt. 


\subsection{Zur Zielerreichung und Wirkung}

Hinsichtlich der Zielerreichung und Wirkung waren im Rahmen der Online-Befragung alle Projektleiterinnen und -leiter der Meinung, dass die HRSM-2013-Projekte ihre grundsätzliche Intention erfüllt haben. Wie in Abbildung 40 dargestellt, wurden (nahezu) alle Projektziele - nach eigenen Angaben der Projektleiterinnen und -leiter erreicht. In fast allen Dimensionen wurde festgehalten, dass die Umsetzung eher bzw. sehr gelungen ist. Die höchste Zielerreichung war dabei bei der „Erhöhung der Sichtbarkeit“ (für 61 \% sehr gelungen), der „Stärkung der Exzellenz" (59 \%) und dem „Aufbau nachhaltiger Kooperationsstrukturen“ (54 \%) zu verzeichnen. Projekten in der Lehre wurde insgesamt eine geringere Zielerreichung zugeordnet. Das mag daran liegen, dass hier Ziele aufgrund der thematischen Schwerpunkte von zahlreichen Projektleiterinnen und -leitern als nicht zutreffend angesehen wurden. Alles in allem sind die Projektleiterinnen und -leiter jedoch überzeugt, die Projektziele gut erreicht zu haben.

Gliedert man die Projekte nach ihrem inhaltlichen Schwerpunkt, so standen teils sehr unterschiedliche Projektziele im Mittelpunkt. Bei Forschungsprojekten sind insbesondere der „Auf- und Ausbau von Forschungsinfrastruktur" und der "Ausbau bestehender Forschungsthemen/Forschungsgruppen“ gelungen. Bei Lehrprojekten wurden speziell die Ziele „Erhöhung der Sichtbarkeit“ und „Weiterentwicklung bestehender Lehrangebote“ erreicht. Bei Projekten, die den inhaltlichen Schwerpunkt der Entwicklung und Erschließung der Künste verfolgten, stachen aufgrund der geringen Fallzahlen keine Ziele hervor, die besonders gelungen sind. Bei Verwaltungsprojekten wurde besonders häufig angegeben, dass die „Erhöhung der Sichtbarkeit“ und die „Verbesserung von Verwaltungsstrukturen/-prozessen“ sehr gelungen sind.

\section{Abbildung 40: Projektziele}

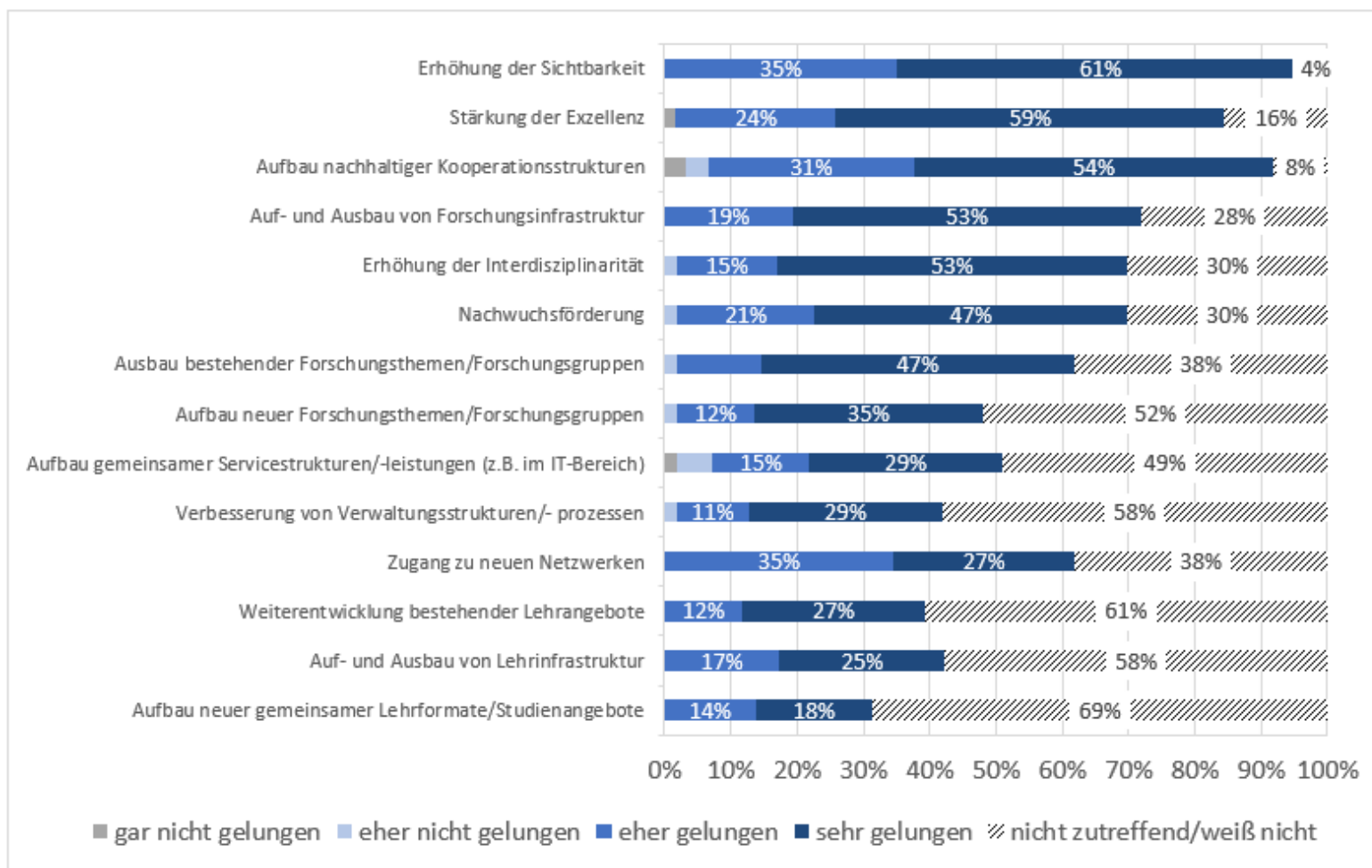

Quelle: WPZ Research Befragung der Projektleiterinnen und -leiter von finanzierten Projekten der HRSM-Ausschreibung 2013, $\mathrm{n}=64$

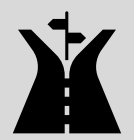

Obwohl die Projekte nicht durch die HRSM-Ausschreibung 2013 finanziert wurden und stattdessen andere Finanzierungsquellen erschlossen werden mussten, konnten die Projekte teils dennoch durchgeführt und zahlreiche Ziele erfüllt werden. So gaben 72 \% der nicht-finanzierten Projektleiterinnen und -leiter an, dass eine 
"Erhöhung der Sichtbarkeit" (eher) gelungen ist. Dahinter folgen der „Zugang zu neuen Netzwerken" und der "Ausbau bestehender Forschungsthemen bzw. Forschungsgruppen" mit starken Zustimmungswerten. Im Vergleich hierzu ist festzuhalten, dass bei den finanzierten Projekten die Zielerreichung in nahezu allen Dimensionen noch besser gelungen ist. Demnach konnten die Universitäten die fehlenden Finanzierungsmittel durch die HRSM-Ausschreibung offenbar nur zum Teil ausgleichen.

\section{Abbildung 41: Projektziele

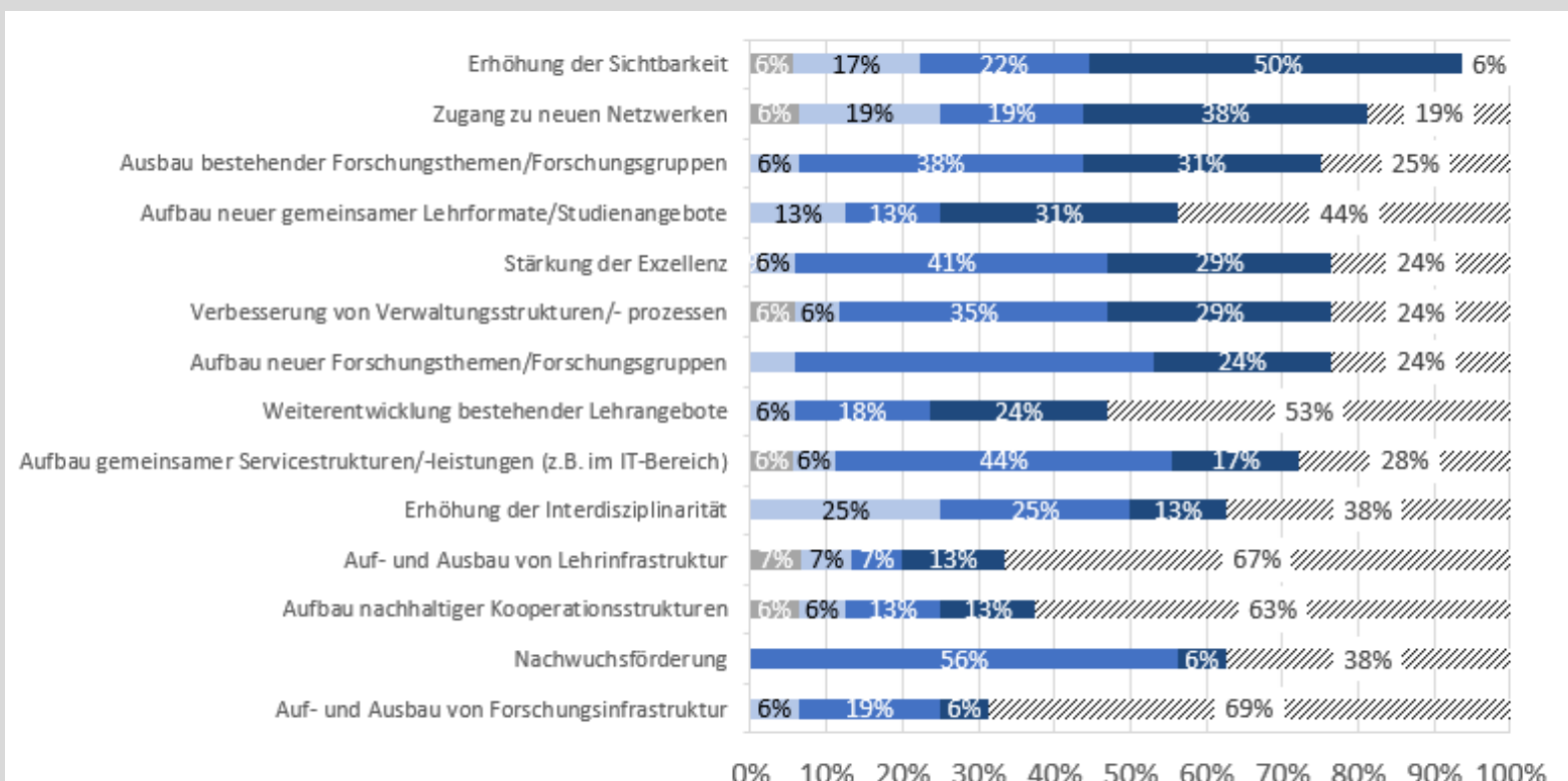

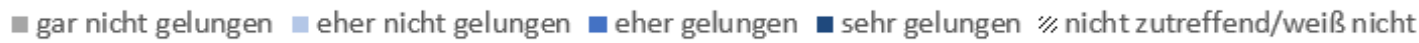

Quelle: WPZ Research Befragung der Projektleiterinnen und -leiter von nicht-finanzierten Projekten der HRSM-Ausschreibung 2013, $n=18$

Werden die Projekte nach ihren inhaltlichen Schwerpunkten gegliedert, zeigen sich z.T. Unterschiede in den erreichten Zielsetzungen. Bei Forschungsprojekten war - auch ohne Finanzierung durch die HRSM - der „Aufbau nachhaltiger Kooperationsstrukturen“ und der "Auf- und Ausbau von Forschungsinfrastruktur" besonders gut gelungen. Von einer Untergliederung in die anderen Schwerpunktbereiche wird aufgrund geringer Fallzahlen Abstand genommen.

Ebenso wie bei den finanzierten Projekten konnten die nicht-finanzierten Projekte nach eigenen Angaben insbesondere ihre Sichtbarkeit verbessern und Synergien nutzen. Mit 50 \% bzw. $45 \%$ waren dies die beiden am häufigsten genannten Zusatznutzen, bei finanzierten Projekten konnten diese Effekte allerdings wesentlich häufiger (83\% bzw. $78 \%$ ) erreicht werden.

Auf die Frage, welchen Zusatznutzen die durch die HRSM 2013 finanzierten Projekte der jeweiligen Universität gebracht haben, antworteten die Projektleiterinnen und -leiter mehrheitlich mit einer verbesserten nationalen oder internationalen Sichtbarkeit und dem Nutzen von Synergieeffekten. Auf diese beiden Zieldimensionen entfielen 83 \% bzw. 78 \%. Dahinter folgten mit deutlichem Abstand das Erzielen eines größeren Leistungsangebots durch Komplementarität, Effizienzgewinne und Schaffen kritischer Größe. 


\section{Abbildung 42: Zusatznutzen}

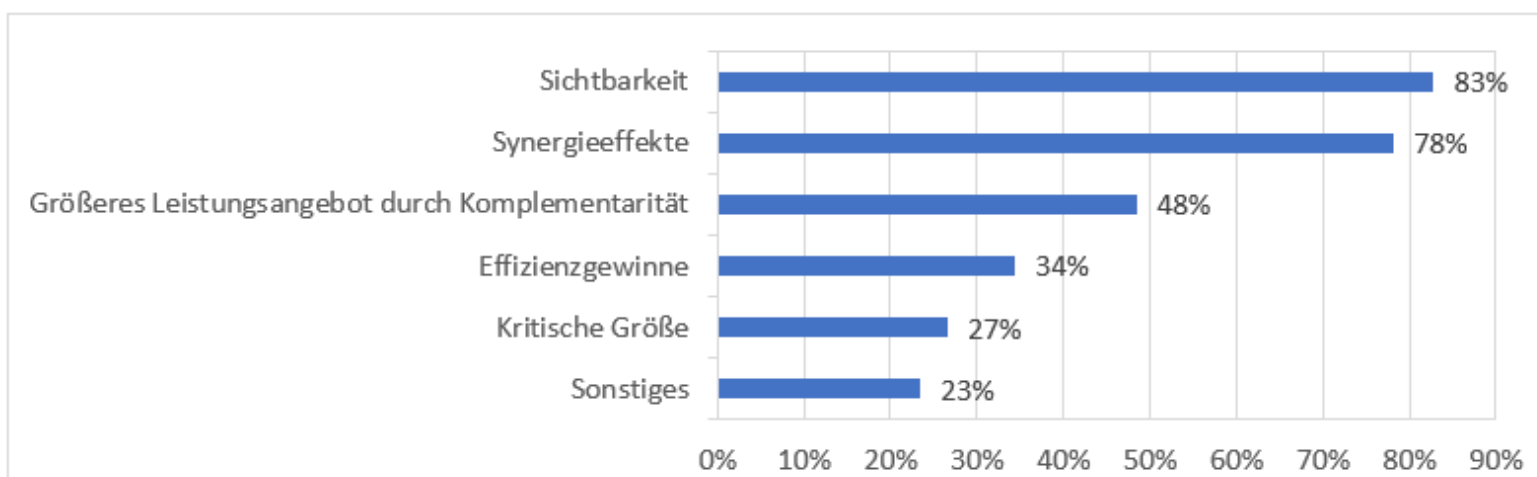

Quelle: WPZ Research Befragung der Projektleiterinnen und -leiter von finanzierten Projekten der HRSM-Ausschreibung 2013, $\mathrm{n}=64$

\section{$\mathbf{Y}$}

Ebenso wie bei den finanzierten Projekten konnten die nicht-finanzierten Projekte nach eigenen Angaben insbesondere ihre Sichtbarkeit verbessern und Synergien nutzen. Mit $50 \%$ bzw. $45 \%$ waren dies die beiden am häufigsten genannten Zusatznutzen, bei finanzierten Projekten konnten diese Effekte allerdings wesentlich häufiger ( $83 \%$ bzw. $78 \%$ ) erreicht werden.

Darüber hinaus nannten 23\% der Projektleiterinnen und -leiter noch weitere Zusatznutzen, die sich aus dem HRSM-Projekt ergeben haben. Ein Auszug davon ist in nachfolgender Box dargestellt.

\section{Statements der Befragten:}

"Attraktivität in der Rekrutierung exzellenter Forscherinnen und Forscher"

"Internationale Anerkennung in der Scientific Community"

"Qualität der Forschung"

"Verbesserte Kontakte zu Politik und Verwaltung"

"Know-How-Aufbau für IKT-Themen, die jetzt relevant sind"

"Steigerung der Qualität des Service-Angebots"

"Gute und viele Veröffentlichungen, Anschlussprojekt (CD-Labor, K-Zentrum)"

Neben den dargestellten Zielen und dem Zusatznutzen gaben die Projektleiterinnen und -leiter auch an, dass die HRSM-Projekte-2013 durchaus auch zur Profilbildung der jeweiligen Universität beigetragen haben. $44 \%$ waren der Meinung, dass das HRSM-Projekt sehr zur Profilbildung beigetragen hat; $42 \%$ gaben an, dass es eher dazu beigetragen hat. 


\section{Abbildung 43: Beitrag zur Profilbildung der Universität}

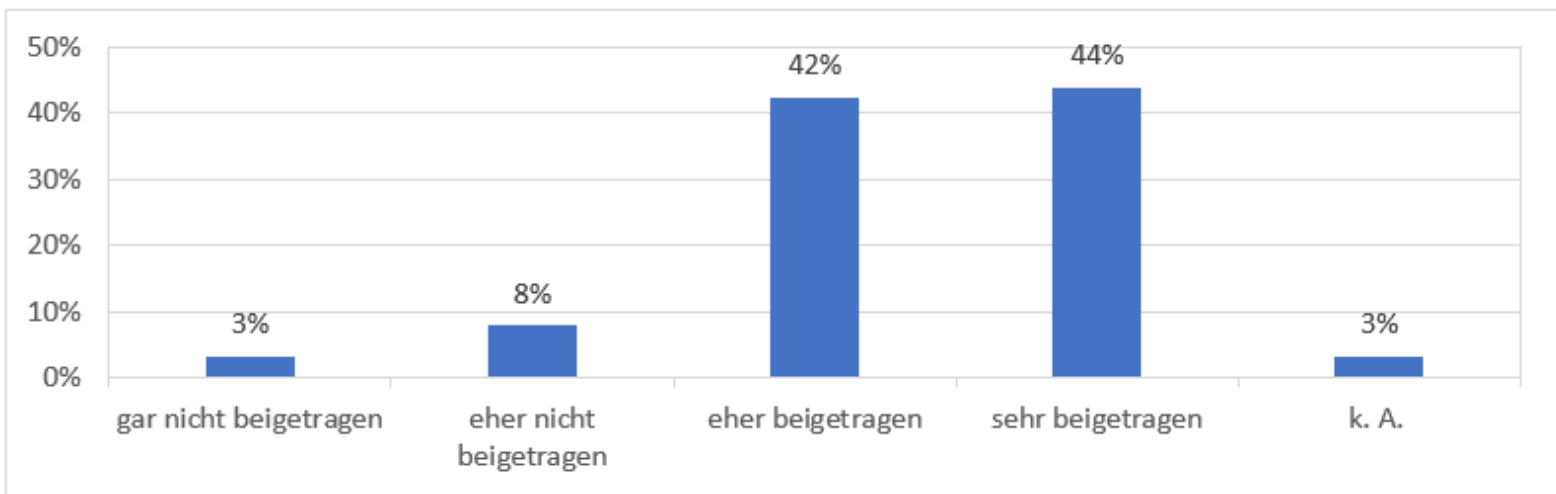

Quelle: WPZ Research Befragung der Projektleiterinnen und -leiter von finanzierten Projekten der HRSM-Ausschreibung 2013, $\mathrm{n}=64$

In einem vergleichbaren Ausmaß von $78 \%$ waren die Projektleiterinnen und -leiter auch der Ansicht, dass ihr HRSM-Projekt (eher/tendenziell) einen Beitrag zu einem abgestimmten Hochschul- und Forschungsraum in Österreich geleistet hat (siehe Abbildung 44).

\section{Abbildung 44: Beitrag zu einem abgestimmten Hochschul- und Forschungsraum}

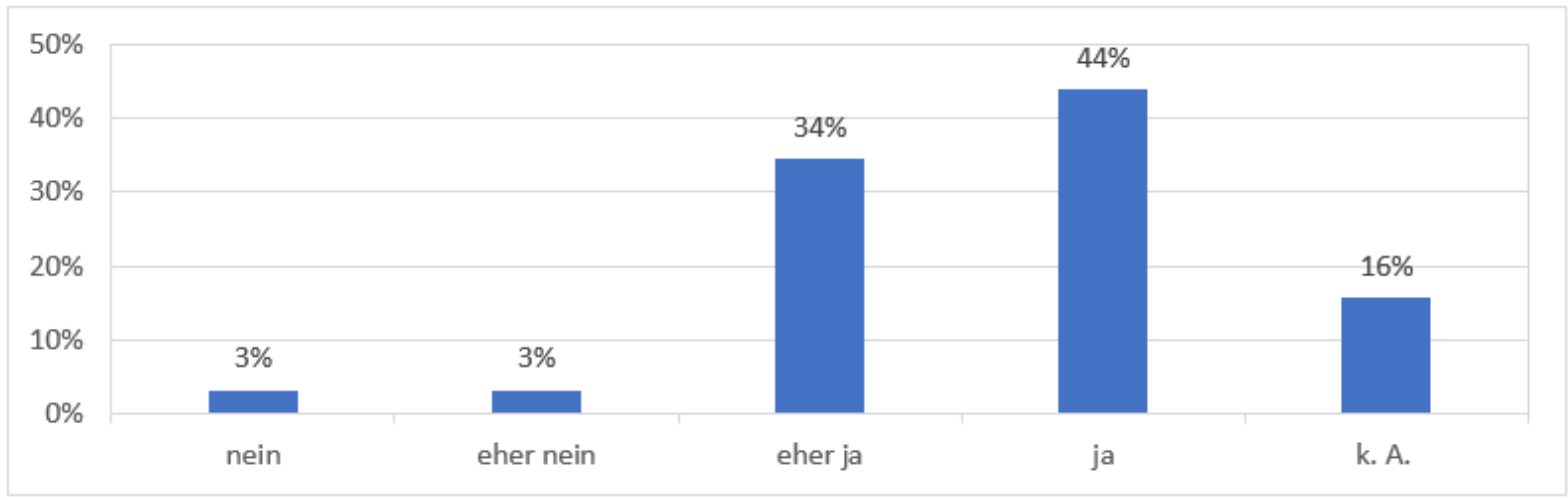

Quelle: WPZ Research Befragung der Projektleiterinnen und -leiter von finanzierten Projekten der HRSM-Ausschreibung 2013, $\mathrm{n}=64$

Im Zuge der Online-Befragung beurteilten die Projektleiterinnen und -leiter die Nachhaltigkeit der durch die HRSM 2013 angestoßenen Kooperationen zu $72 \%$ als (sehr) hoch, zu $23 \%$ als mittel und nur $2 \%$ sehen eine (sehr) niedrige Nachhaltigkeit der Kooperationen. Daraus kann geschlossen werden, dass die HRSM über ihre Laufzeit hinaus eine positive Wirkung auf die jeweilige Universität entfaltet und einen längerfristigen Beitrag geleistet haben. Zwischen den verschiedenen inhaltlichen Schwerpunkten bestehen in puncto Nachhaltigkeit geringe Unterschiede. Bei Forschungsprojekten wurde eine höhere Nachhaltigkeit angegeben, bei Projekten der Entwicklung und Erschließung der Künste eine etwas geringer, wobei dieser Schwerpunkt aufgrund der geringen Fallzahlen mit Vorsicht zu interpretieren ist. 


\section{Abbildung 45: Nachhaltigkeit}

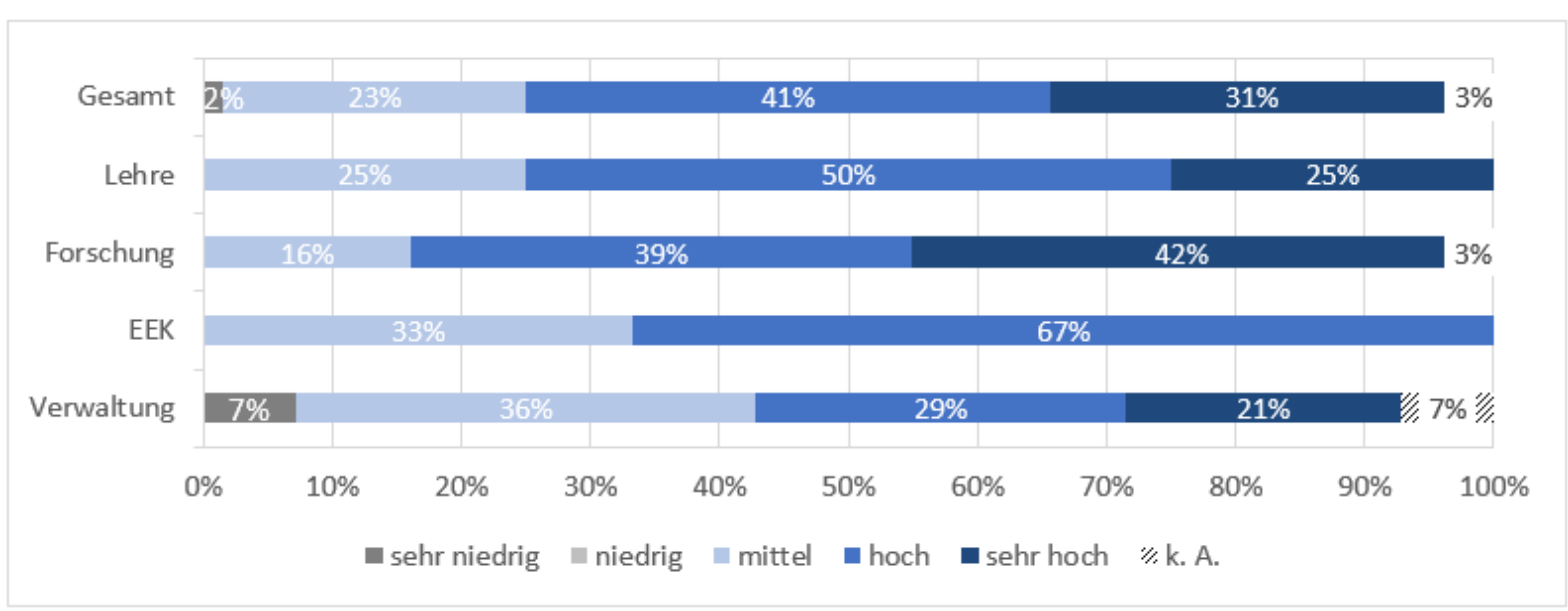

Quelle: WPZ Research Befragung der Projektleiterinnen und -leiter von finanzierten Projekten der HRSM-Ausschreibung 2013, $\mathrm{n}=64$

Auffällig ist, dass sich die Einschätzung der Nachhaltigkeit zum Teil nach Universitätstyp stark unterscheidet. Nur die Hälfte aller befragten Projektleiterinnen und -leiter an Kunstuniversitäten vertritt die Meinung, dass die Nachhaltigkeit ihres HRSM-Projekts (sehr) hoch gewesen sei. Bei den Sonstigen Universitäten sind es dagegen mit $88 \%$ fast alle. Die übrigen Universitätstypen zeigen kein abweichendes Antwortverhalten. Offenbar existieren unterschiedliche universitätsinterne Strukturen, welche die Nachhaltigkeit begünstigen oder eben erschweren.

Die Nachhaltigkeit wurde, laut Angaben der Projektleiterinnen und -leiter, insbesondere dadurch sichergestellt, dass neben den HRSM-Projekten weitere Kooperationsprojekte initiiert und gestartet wurden. $64 \%$ nannten dies als Erklärung. Dahinter folgten Veränderungen von universitären Strukturen bzw. Prozessen (41 \%), die Verankerung der Projekte in der Leistungsvereinbarung (38 \%) und die Einwerbung von zusätzlichen Finanzierungsmitteln (36 \%). In die Antwortkategorie „Sonstiges" fielen etwa Spin-offs, die Schaffung von Professuren oder die weiterführende Nutzung von angeschaffter Infrastruktur.

\section{Abbildung 46: Sicherstellung der Nachhaltigkeit}

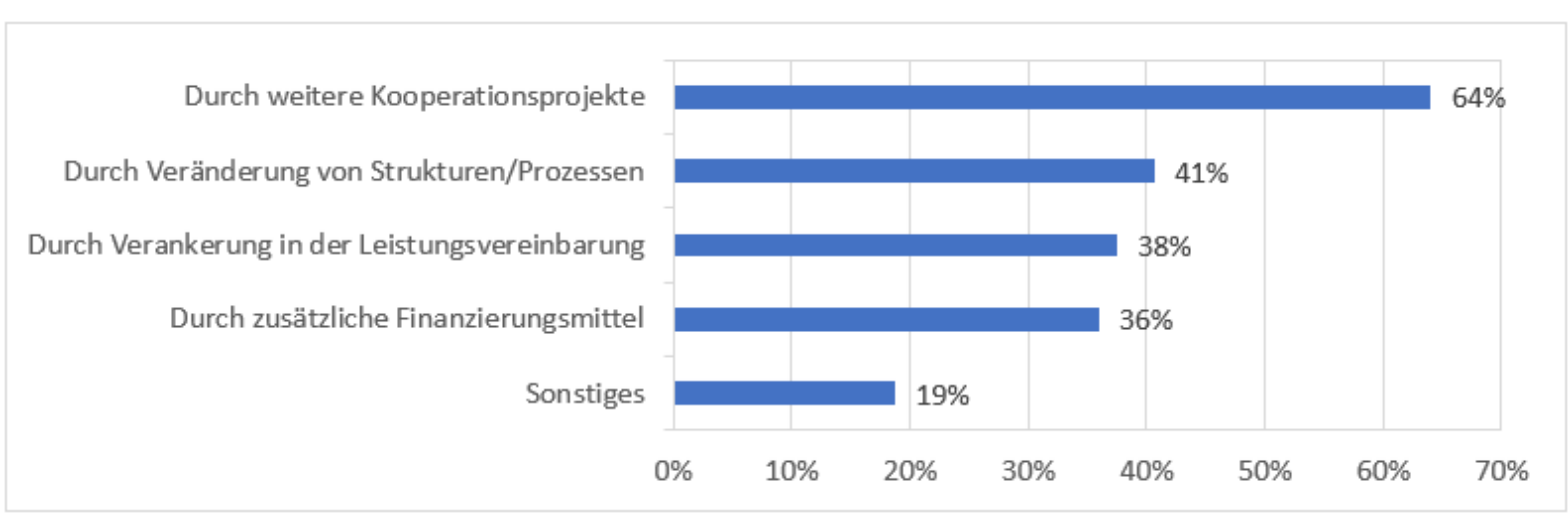

Quelle: WPZ Research Befragung der Projektleiterinnen und -leiter von finanzierten Projekten der HRSM-Ausschreibung 2013, $\mathrm{n}=64$

Insgesamt wurden die ohne HRSM durchgeführten Projekte - es handelt sich hierbei um Befunde zu jenen Projekten, deren Leiterinnen und Leiter an der Umfrage teilnahmen - zu $45 \%$ als nachhaltig beurteilt; $40 \%$ sahen die Nachhaltigkeit zumindest als teilweise gegeben; $15 \%$ waren der Meinung, dass ihr Projekt nicht 
nachhaltig gewirkt hat (siehe Abbildung 47). Mit $60 \%$ wurde diese Nachhaltigkeit am ehesten dadurch erreicht, dass universitätsinterne Prozesse bzw. Strukturen verändert wurden. Bei den finanzierten Projekten waren es im Vergleich hierzu am häufigsten weitere Nachfolgeprojekte, welche die Nachhaltigkeit sicherstellten. Sofern eine Untergliederung nach inhaltlichen Schwerpunkten aufgrund der geringen Fallzahlen sinnvoll ist, zeigen sich nur geringe Unterschiede zwischen Projekten mit Fokus auf Lehre, Forschung, Entwicklung und Erschließung der Künste bzw. Verwaltung.

\section{Abbildung 47: Nachhaltigkeit}

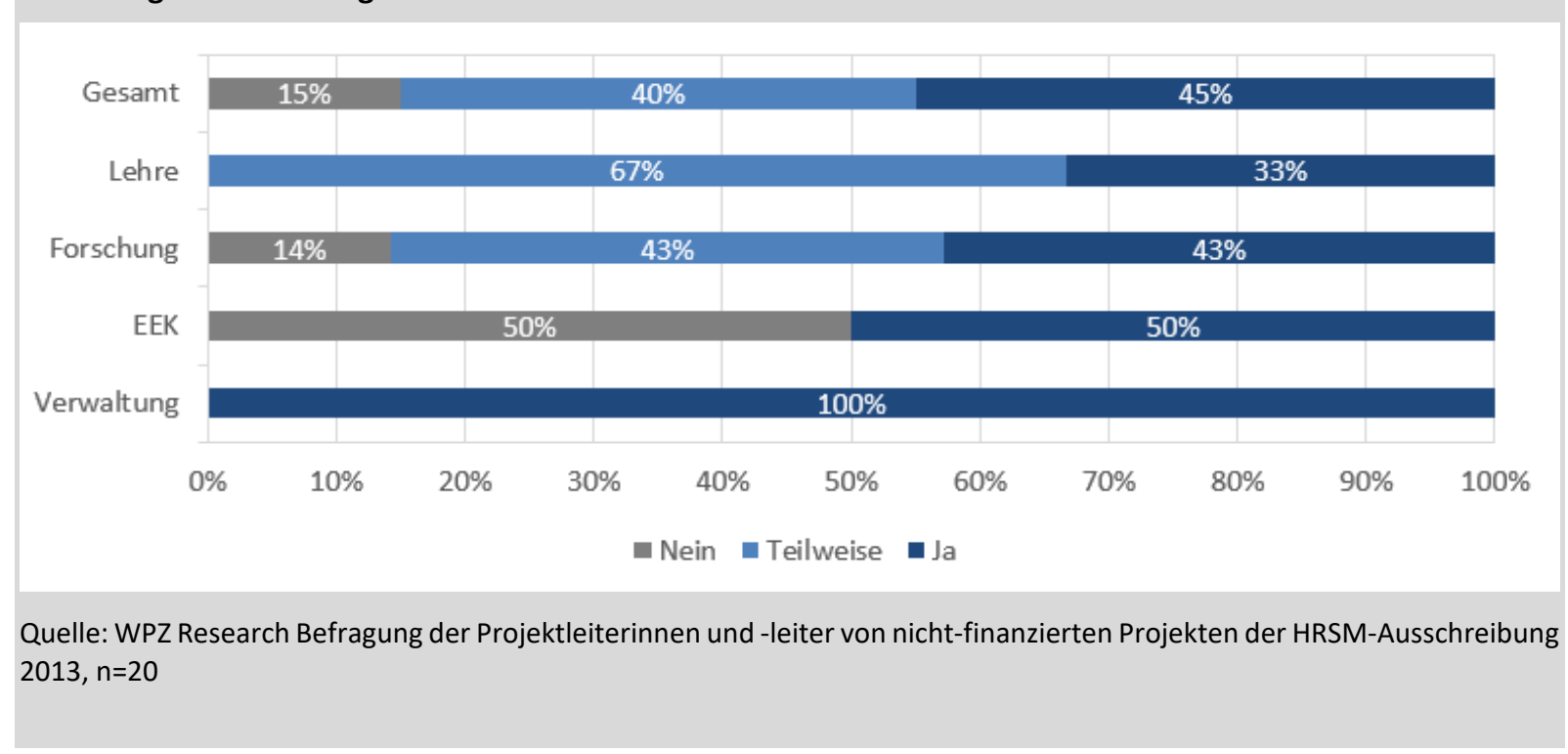

Hätten die Kooperationsprojekte der Universitäten keine Finanzierung durch die HRSM erhalten, so wären sie in 31 \% der Fälle gar nicht durchgeführt worden. 67 \% der Projektleiterinnen und -leiter gaben an, dass ohne die HRSM-Finanzierung ihr Projekt womöglich zu Stande gekommen wäre, allerdings in einem geringeren Umfang. Mit 2 \% gab nur ein verschwindend geringer Anteil an, dass das jeweilige Kooperationsprojekt auch gänzlich ohne Finanzierung durch das BMBWF zu Stande gekommen wäre. Damit wird klar, dass durch die HRSM-Finanzierung Kooperationsprojekte an Österreichs Universitäten angestoßen und durchgeführt wurden, die ansonsten nicht im selben Umfang bzw. nicht hätten durchgeführt werden können. Zwischen den verschiedenen inhaltlichen Schwerpunkten bestehen diesbezüglich keine Unterschiede im Antwortverhalten. Werden die Universitätstypen miteinander verglichen, so zeigt sich, dass vermehrt die Sonstigen Universitäten der Meinung sind, dass sie ihr Projekt auch ohne HRSM-Finanzierung hätten durchführen können. Die Leiterinnen und Leiter der Volluniversitäten sind eher der Meinung, dass ihr Projekt auch ohne HRSM umgesetzt hätte werden können.

\section{Abbildung 48: Kooperationen auch ohne die HRSM-Finanzierung bzw. das HRSM-Projekt}

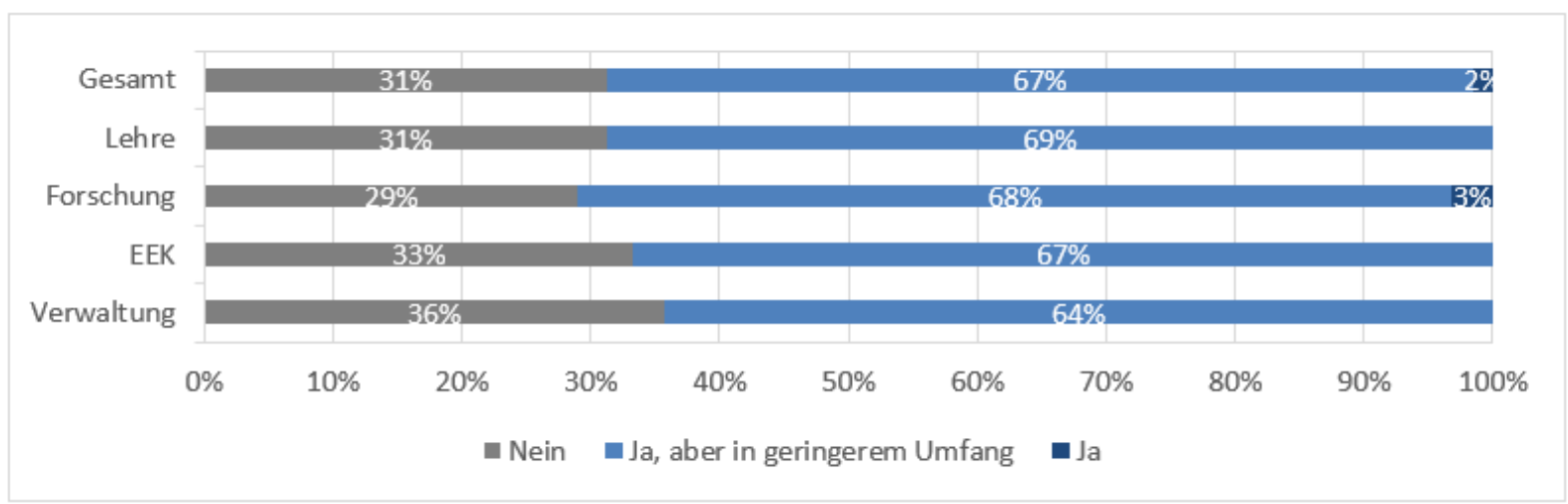

Quelle: WPZ Research Befragung der Projektleiterinnen und -leiter von finanzierten Projekten der HRSM-Ausschreibung 2013, $\mathrm{n}=64$ 
Alles in allem würden $86 \%$ der befragten Projektleiterinnen und -leiter wieder an einer zukünftigen HRSMAusschreibung bzw. einer vergleichbaren Ausschreibung teilnehmen. $11 \%$ würden es eher tun. Keine Projektleiterin bzw. kein -leiter würde sich aufgrund der gesammelten Erfahrungen (eher) nicht mehr an einer solchen Ausschreibung beteiligen.

\section{Abbildung 49: Teilnahme an einer zukünftigen HRSM-Ausschreibung bzw. einer vergleichbaren Ausschreibung}

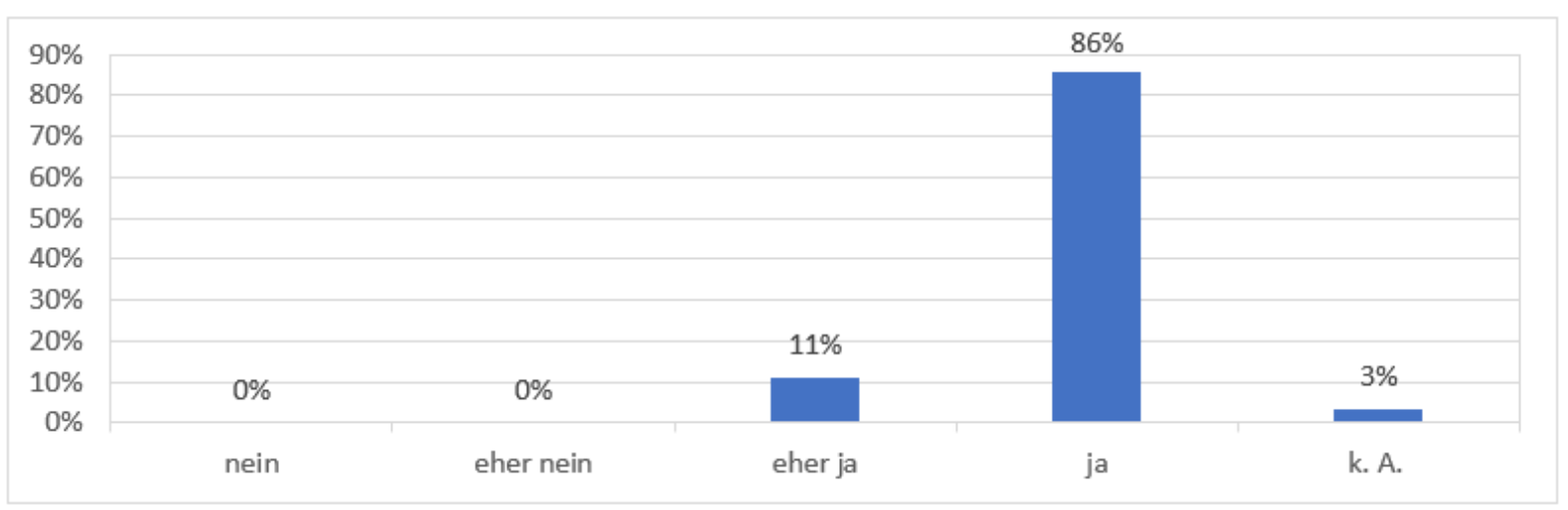

Quelle: WPZ Research Befragung der Projektleiterinnen und -leiter von finanzierten Projekten der HRSM-Ausschreibung 2013, $\mathrm{n}=64$

Auch wenn die befragten Personen schließlich keine finanziellen Mittel aus der HRSM-Ausschreibung 2013 lukrieren konnten, würden dennoch 75 \% bei einer zukünftigen bzw. vergleichbaren Ausschreibung wieder teilnehmen. 22 \% würden sich dagegen (eher) kein weiteres Mal an einer solchen Ausschreibung beteiligen wollen.

Abbildung 50: Teilnahme an einer zukünftigen HRSM-Ausschreibung bzw. einer vergleichbaren Ausschreibung

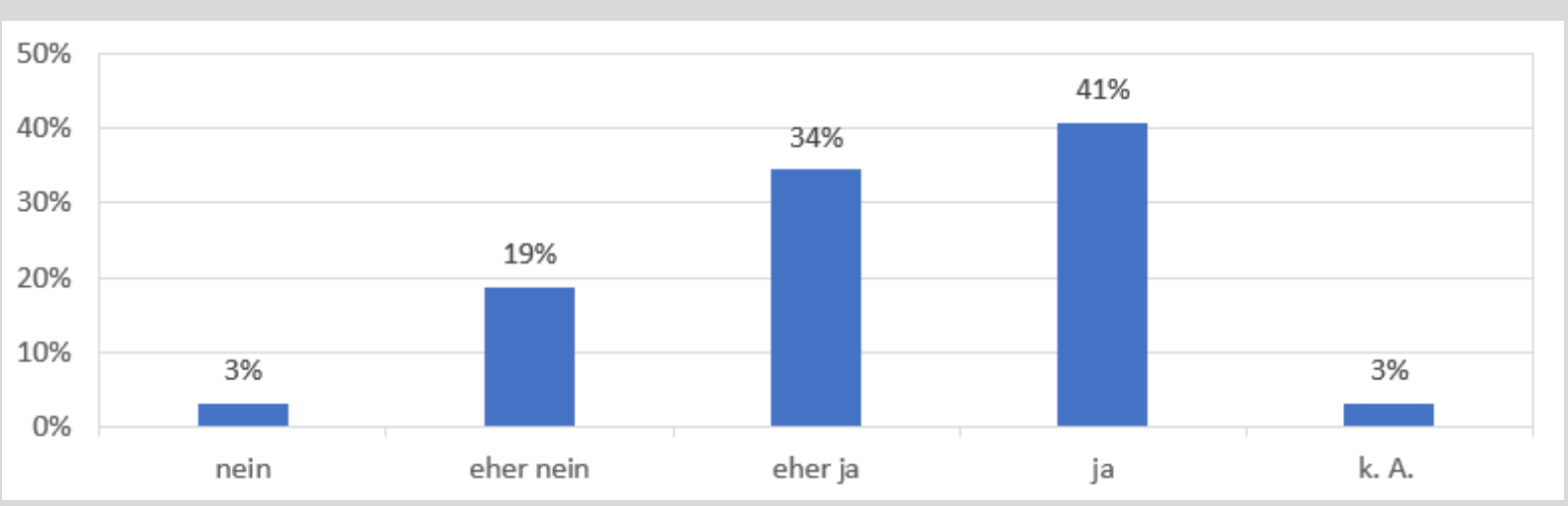

Quelle: WPZ Research Befragung der Projektleiterinnen und -leiter von nicht-finanzierten Projekten der HRSM-Ausschreibung 2013, n=32 
Einige Projektleiterinnen und -leiter nutzten die Online-Umfrage auch, um ihre insgesamt positive Sicht auf die HRSM-Projekte-2013 mit eigenen Worten zu schildern und ein Fazit zu ziehen:

\section{Statements der Befragten:}

"ICh halte die HRSM-Ausschreibung für ein sehr wichtiges Element, um universitätsübergreifende Forschungsinfrastrukturen aufzubauen bzw. zu stärken. Erfolgreiche Aktivitäten sollten in den Leistungsvereinbarungsgesprächen behandelt werden, um den langfristigen Nutzen der Investitionen sicherzustellen."

„Die HRSM-Mittel sind ein wesentlicher Baustein zur Stärkung der Großgeräteinfrastruktur und sollten daher weitergeführt werden."

„Es wäre schön, falls es für erfolgreich durchgeführt HRSM-Projekte eine Weiterführung/Extension gäbe."

„Wir waren und sind sehr dankbar für diese Möglichkeit der qualitätsgesicherten multidisziplinären Forschung mit Motivationsförderung zur Intensivierung der Zusammenarbeit zwischen verschiedenen Universitäten. Das Instrument sollte absolut ausgebaut werden, indem (1) die Fördersumme gesamt massiv erhöht wird, und dadurch (2) entweder wesentlich mehr Projekte bewilligt werden können und/oder (3) die Bewilligungsquote steigt. Implizit handelt es sich auch um ein Instrument der Nachwuchsförderung und Frauenförderung, da hier auch Personen maßgeblich Mittel aufstellen können, die nicht ganz oben in der Universitätshierarchie sind und keine zentralen Personen universitärer Entscheidungsprozesse sind."

„Sehr hilfreiches Instrument, um nachhaltige Kooperationen zu fördern, welche in anderen Förderschienen nicht abgedeckt sind."

"Fazit: Die 2/3 an Eigenleistung stellen Universitäten und auch die Partnerinnen und Partner vor finanzielle Probleme."

„Die Ausschreibung(en) sind wertvoll und eine Weiterführung wäre sehr wichtig für den Zukunftsstandort Österreich." 


\section{HRSM-Ausschreibung 2016 für Kooperationen im Bereich Lehre}

Ein besonderer Schwerpunkt der HRSM-Ausschreibung 2016 im Bereich Lehre lag auf der Entwicklung und Umsetzung der PädagogInnenbildung Neu in vier Entwicklungsverbünden (West, Mitte, Nord-Ost und Süd-Ost). Durch diese Reform sollen Lehramtsstudien für allgemeinbildende Fächer an den Schulen der Sekundarstufe (Mittelschulen, Allgemeinbildende Höhere Schulen, Polytechnische Schulen, Mittlere und Höhere Berufsbildende Schulen) gemeinsam von den öffentlichen Universitäten und Pädagogischen Hochschulen entwickelt und durchgeführt werden. Die Umsetzung soll in Form eines vier Jahre dauernden Bachelorstudiums, an das ein Masterstudium anschließt, das zumindest 1,5 Jahre dauert, geschehen. Der zweite Bereich der Ausschreibung war den Sonstigen Lehrprojekten gewidmet, die als Impulsgeber für verstärkte Kooperationen in der Lehre dienen sollten.

Für die HRSM-Ausschreibung 2016 im Bereich Lehre standen insgesamt 35 Mio. Euro, welche im Rahmen eines kompetitiven Verfahrens vom BMBWF vergeben wurden, als Anschubfinanzierung für strategische Kooperationsprojekte an Österreichs Universitäten zur Verfügung. Der gesamten Ausschreibung (Lehre, Forschung/EEK, Verwaltung) standen 97,5 Mio. Euro zur Verfügung. Während 2013 nur rund 5,5 Mio. Euro (bzw. $9 \%$ der Gesamtmittel) in den Bereich Lehre flossen, kamen rund 36 \% der verfügbaren Mittel 2016 dem Bereich Lehre zugute. Verglichen mit 2013 ist daher erkennbar, dass der Bereich Lehre in der HRSM-Ausschreibung 2016 zentraler ist - mit einem besonderen Schwerpunkt auf das bildungspolitische Vorhaben der PädagogInnenbildung Neu. Der Start der finanzierten Projekte begann mit einer Ausnahme im Jahr 2016; alle Projekte liefen drei bis vier Jahre (Projektende 2018 bzw. 2019).

Im Folgenden wird die HRSM-Ausschreibung 2016 für Kooperationen im Bereich Lehre analysiert, wobei zuerst der Prozess der Antragsstellung, dann die konkrete Umsetzung der Projekte und abschließend die Zielerreichung und Wirkung im Mittelpunkt stehen. Dazu werden die methodischen Instrumente Analyse der Projektanträge, Online-Befragung, Portfolioanalyse und Netzwerkanalyse miteinander kombiniert und verschränkt.

\subsection{Zur Antragsstellung}

In der Phase der Antragstellung stehen der Antragsprozess, die Motive zur Teilnahme, der Aufwand für die Antragstellung sowie die Vergabeentscheidung im Fokus der Betrachtung.

\subsubsection{Antragsprozess}

Im Rahmen der Befragung der Projektleiterinnen und -leiter (siehe hierzu auch Kapitel 1.3 Herangehensweise und Methodik) gaben alle Befragten an, dass die Initiative zur Antragsstellung von ihrer Universität ausging. In keinem Fall war das Engagement einer Kooperationspartnerin bzw. eines Kooperationspartners ausschlaggebend. Wie in Abbildung 51 dargestellt, haben $41 \%$ der Projektleiterinnen und -leiter ein bis drei Monate vor der Einreichung ihre Anträge vorbereitet. $26 \%$ taten dies drei bis sechs Monate davor und $22 \%$ kurzfristig in einer Periode von weniger als einem Monat vor der Antragsstellung.

\section{Abbildung 51: Vorbereitung der Antragsstellung}

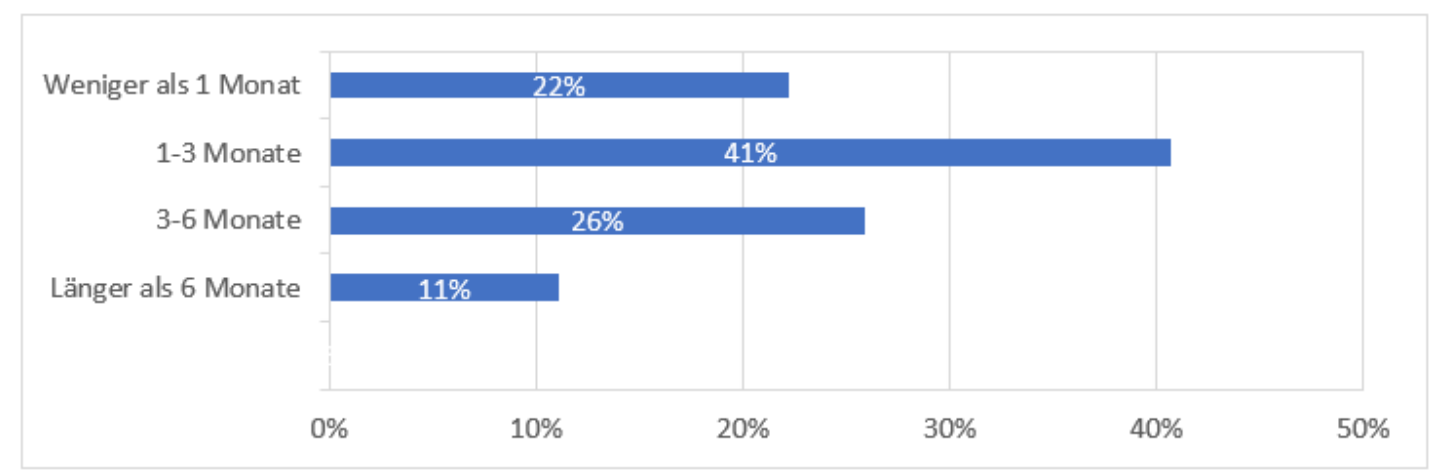

Quelle: WPZ Research Befragung der Projektleiterinnen und -leiter von finanzierten Projekten der HRSM-Ausschreibung 2016, $\mathrm{n}=27$ 


\subsubsection{Motive zur Teilnahme}

Die Gründe für die Teilnahme an der HRSM-Ausschreibung 2016 waren vielfältig. 56 \% der Projektleiterinnen und -leiter gaben an, dass sie den Aufbau nachhaltiger Kooperationsstrukturen angestrebt haben. Dahinter folgten die Gewährleistung und Verbesserung der Qualität der Lehre in Lehramtsstudien (48 \%) und der Aufbau neuer universitäts- und hochschulübergreifender Studienangebote (inkl. Lehramtsstudien) (44\%). Die Gründe, die am seltensten angeführt wurden, sind die Verbesserung der innerösterreichischen Mobilität von Studierenden (11\%), die Förderung von Frauen (11\%) und der Ausbau von MINT-Fächern (7\%). Differenziert man zwischen den beiden inhaltlichen Schwerpunkten Pädagoginnen- und Pädagogenbildung Neu und sonstigen Lehrprojekten, zeigen sich klare Unterschiede in den Gründen für die Teilnahme. Leiterinnen und Leiter von Projekten, die einen Schwerpunkt auf der Pädagoginnen- und Pädagogenbildung Neu hatten, gaben insbesondere an, an der HRSM-Ausschreibung 2016 teilgenommen zu haben, um die Qualität der Lehre in Lehramtsstudien zu gewährleisten bzw. weiter zu verbessern und um den Aufbau neuer institutionsübergreifender Studienangebote voranzutreiben. Bei sonstigen Lehrprojekten waren die Gründe für die Teilnahme vielfältiger, sie umfassten sowohl den Aufbau nachhaltiger Kooperationsstrukturen, den Einsatz von E-Learning Formaten, die Digitalisierung der Lehre als auch die Erhöhung der Diversität.

\section{Abbildung 52: Gründe für die Teilnahme an der HRSM-Ausschreibung 2016 im Bereich Lehre}

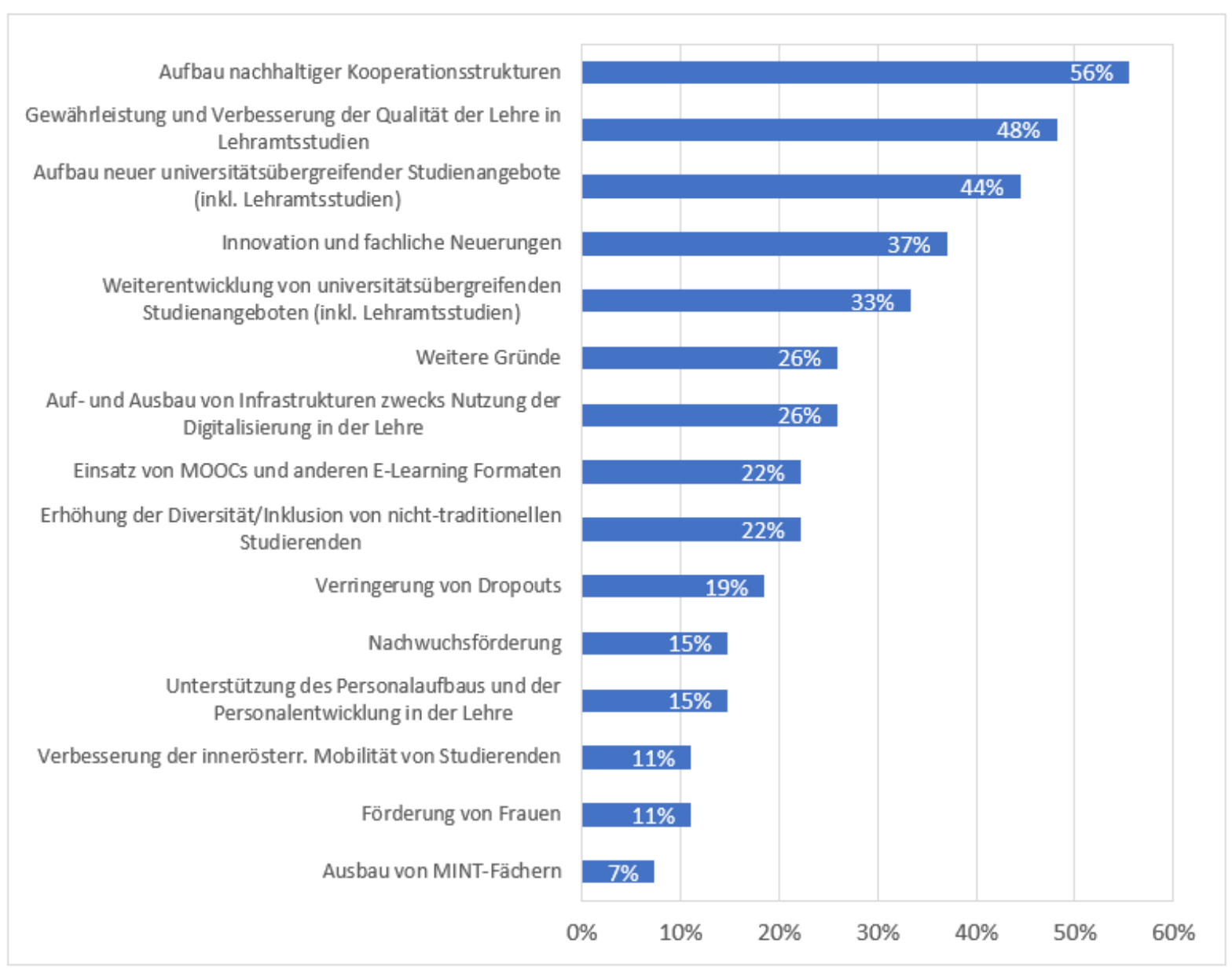

Quelle: WPZ Research Befragung der Projektleiterinnen und -leiter von finanzierten Projekten der HRSM-Ausschreibung 2016, $\mathrm{n}=27$

\subsubsection{Aufwand und Angemessenheit}

Mit $96 \%$ gaben fast alle Projektleiterinnen und -leitern in der Online-Befragung an, dass die Ausschreibungsunterlagen in Bezug auf Ziele und Anforderungen nachvollziehbar gewesen seien. Lediglich $4 \%$ waren anderer Meinung. Insgesamt ist dies als äußerst positiv zu beurteilen (siehe Abbildung 53). 
Abbildung 53: Nachvollziehbarkeit der Ausschreibungsunterlagen in Bezug auf Ziele und Anforderungen

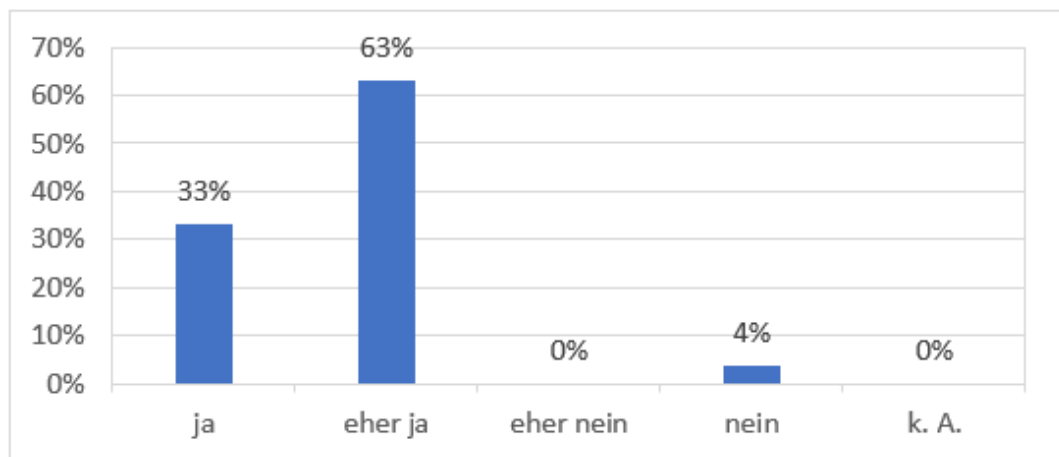

Quelle: WPZ Research Befragung der Projektleiterinnen und -leiter von finanzierten Projekten der HRSM-Ausschreibung 2016, $n=27$

Der administrative Aufwand für die Antragsstellung war für 44 \% der Projektleiterinnen und -leiter mittel, 30 \% schätzten ihn als hoch ein und $15 \%$ sogar als sehr hoch. Im Vergleich dazu waren es bei der HRSM-Ausschreibung 2013 lediglich 5 \% gewesen, die den Aufwand als sehr hoch bewerteten. Bei Projekten, die der Pädagoginnenund Pädagogenbildung Neu zugeordnet werden können, wurde der administrative Aufwand seitens der Leiterinnen und Leiter höher eingeschätzt als bei sonstigen Lehrprojekten.

\section{Abbildung 54: Administrativer Aufwand für die Antragsstellung}

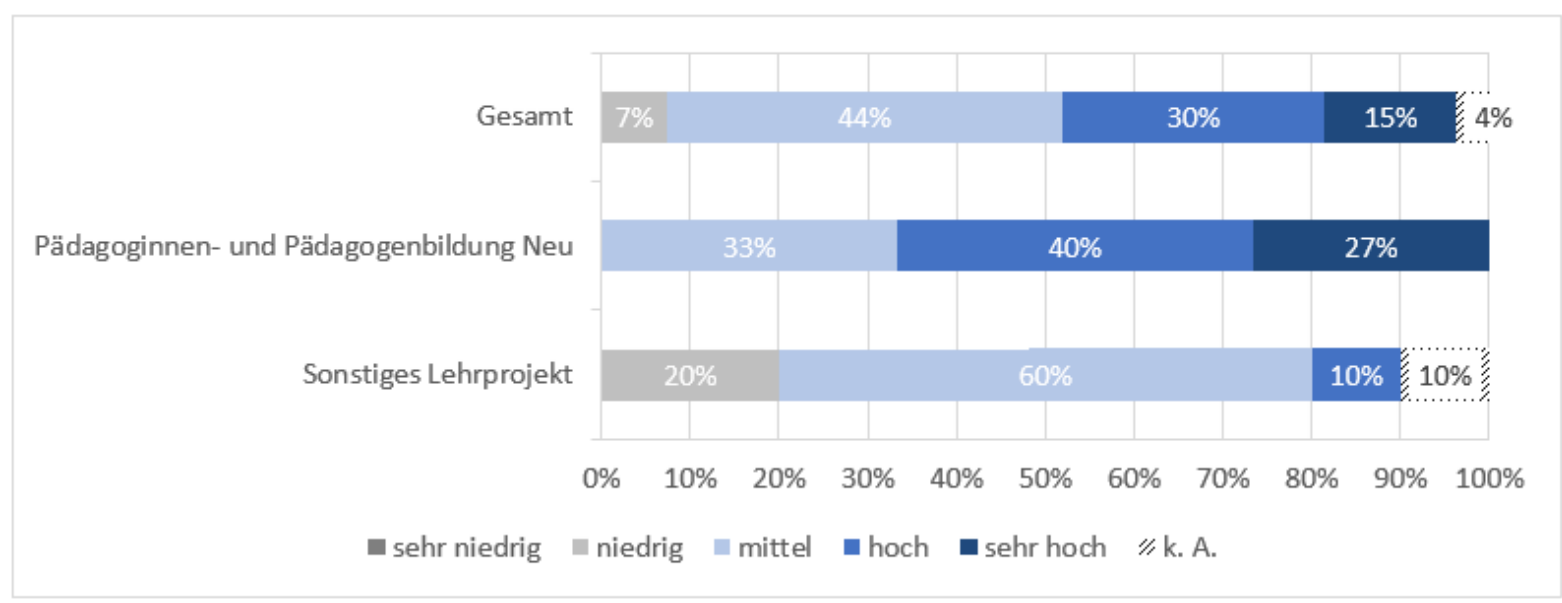

Quelle: WPZ Research Befragung der Projektleiterinnen und -leiter von finanzierten Projekten der HRSM-Ausschreibung 2016, $\mathrm{n}=27$

Im Zuge des Antragsverfahrens für die HRSM 2016 haben 44 \% der Projektleiterinnen und -leiter den Helpdesk des BMBWF in Anspruch genommen, das waren um 10 Prozentpunkte mehr als bei der Ausschreibung 2013. Mit $58 \%$ standen dabei die inhaltlichen Fragen etwas mehr im Mittelpunkt als die technischen Fragen. Die Unterstützung von Seiten des BMBWF wurde zu $67 \%$ als sehr hilfreich und zu $33 \%$ als eher hilfreich eingeschätzt. Die Projektleiterinnen und -leiter stellen dem Helpdesk des BMBWF damit ein sehr gutes Zeugnis aus.

Im Rahmen der Antragsstellung mussten Bewertungskriterien („Indikatorenset und Selbsteinschätzung“) angegeben werden. Die Bewertungskriterien für Projekte der PädagogInnenbildung Neu umfassten: Etablierung gemeinsamer Strukturen im Bereich des Lehrens und Lernens für nachhaltige Kooperationen auch über die konkrete Projektdauer hinaus; Sicherung und Weiterentwicklung der wissenschaftlichen und professionsorientierten Voraussetzungen durch die Schaffung kooperativer, insbesondere auch hochschulübergreifender Arbeitseinheiten in den Verbundregionen zur Durchführung der Lehramtsstudien unter besonderer Berücksichtigung des zu erwartenden Bedarfs an Absolventinnen und Absolventen; Einrichtung und Weiterentwicklung von Lehramtsstudien unter Nutzung sämtlicher vorhandener Ressourcen innerhalb einer Verbundregion und ggf. über diese hinausgehend unter besonderer Berücksichtigung des zu erwartenden 
Bedarfs an Absolventinnen und Absolventen; Gewährleistung einer qualitätsvollen Lehre in besonders frequentierten Lehramtsstudien unter Berücksichtigung des Bedarfs an Absolventinnen und Absolventen; Förderung des wissenschaftlichen Nachwuchses, insbesondere für die Fachdidaktiken und den Primarbereich (z.B. Doktorandenkollegs, (Verbund-)Professuren); Innovation und fachliche Neuerungen, wie z.B. fächerübergreifende Angebote zur Förderung von MINT-Fächern etc.

Die Bewertungskriterien für sonstige Lehrprojekte umfassten: Etablierung gemeinsamer Strukturen im Bereich des Lehrens und Lernens für nachhaltige Kooperationen auch über die konkrete Projektdauer hinaus; Entwicklung universitätsübergreifender Studienangebote unter Nutzung vorhandener Ressourcen und Synergien; konkrete Maßnahmen zur Erhöhung der Studienaktivität und der Abschlussquoten; institutions- bzw. organisationsübergreifende Integration und Betreuung nicht-traditioneller Studierender (z.B. berufsbegleitendes Studium, Förderung von Studierenden aus bildungsfernen Schichten, Förderung der gleichen Teilhabe von Männern und Frauen an einzelnen Studienfächern (insb. MINT-Fächer); Innovationen/Optimierungen im Bereich von IT-basierten Lehr- und Lernformaten; sowie Erleichterung der innerösterreichischen Mobilität von Studierenden.

Die Passgenauigkeit bzw. die Angemessenheit dieser Bewertungskriterien wurde von einer Mehrheit von $63 \%$ der befragten Projektleiterinnen und -leiter als hoch eingeschätzt, von $11 \%$ sogar als sehr hoch. $15 \%$ schätzten die Passgenauigkeit bzw. die Angemessenheit als mittel und lediglich $4 \%$ als niedrig ein. Zwischen Projekten mit einem Schwerpunkt auf Pädagoginnen- und Pädagogenbildung Neu und sonstigen Lehrprojekten sind kaum Unterschiede im Antwortverhalten zu verzeichnen.

Abbildung 55: Passgenauigkeit/Angemessenheit der Bewertungskriterien („Indikatorenset und Selbsteinschätzung") der Ausschreibung

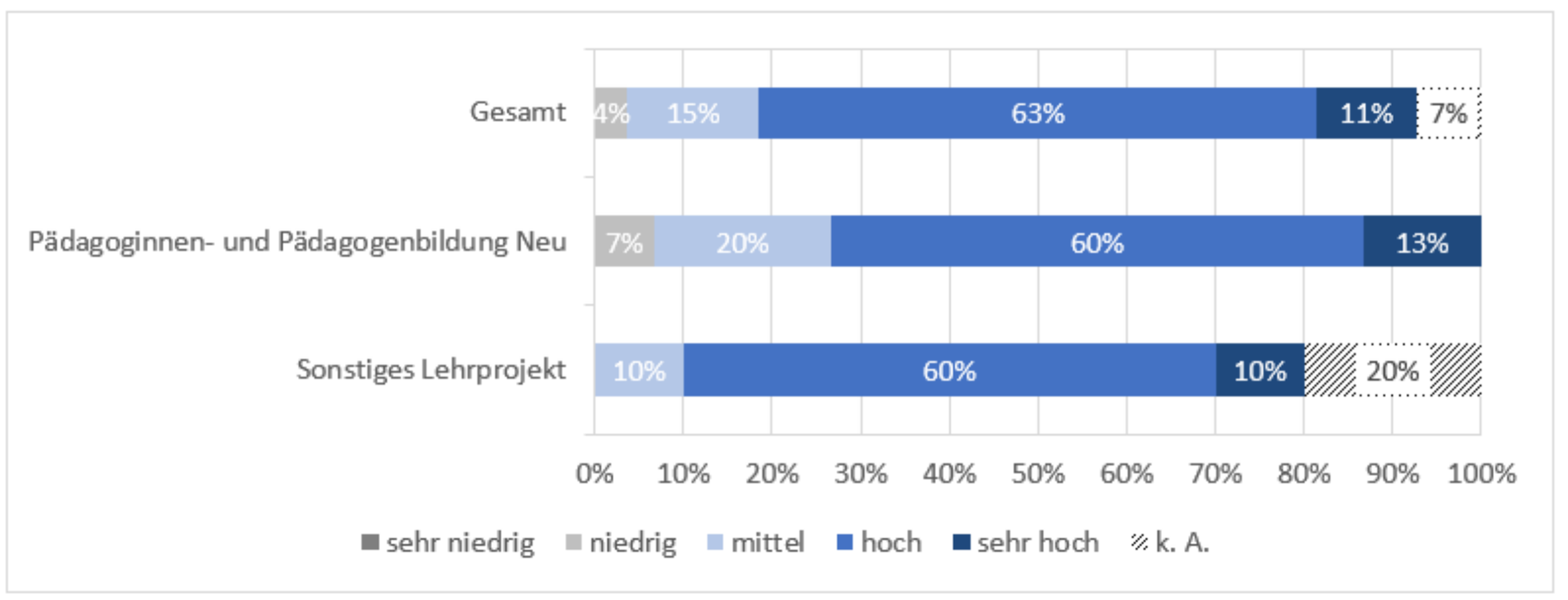

Quelle: WPZ Research Befragung der Projektleiterinnen und -leiter von finanzierten Projekten der HRSM-Ausschreibung 2016, $\mathrm{n}=27$

Im Zuge der Antragsstellung mussten ebenso Bezüge zur Leistungsvereinbarung 2013-2015 bzw. zum Entwicklungsplan der jeweiligen Universität angegeben werden. Fast allen Projektleiterinnen und -leitern fiel dies (sehr) leicht (89\%); lediglich $4 \%$ gaben an, dies als schwierig empfunden zu haben (siehe Abbildung 56). 
Abbildung 56: Bezug zur Leistungsvereinbarung 2013-2015 bzw. zum Entwicklungsplan der Universität

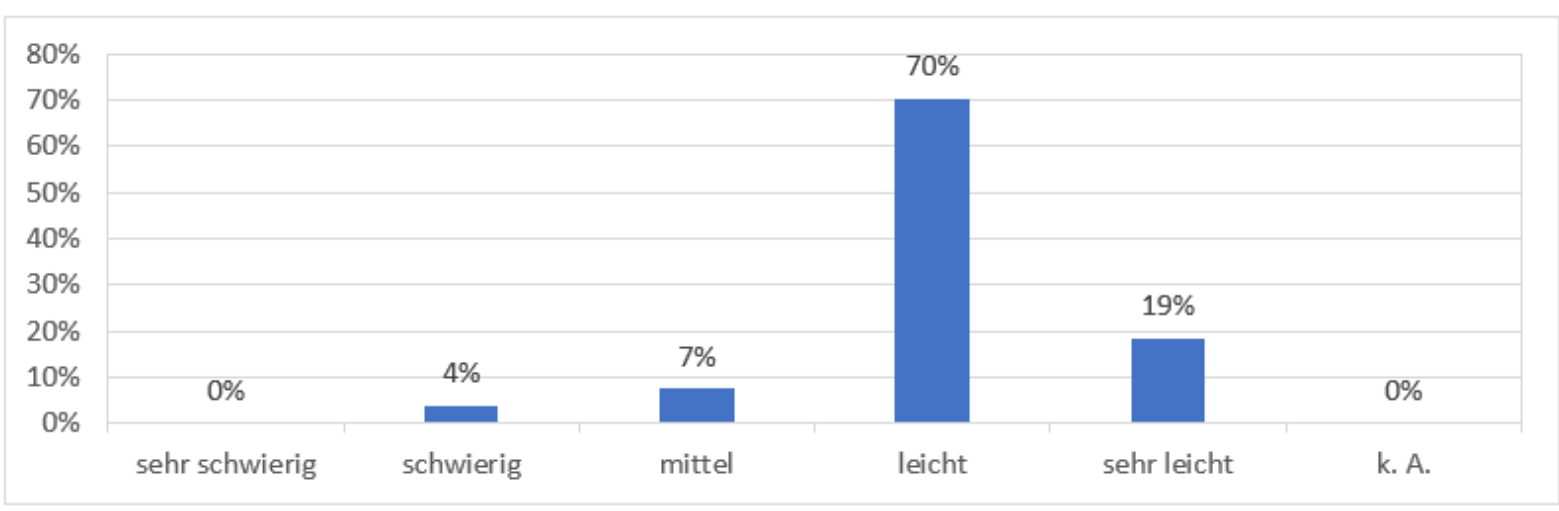

Quelle: WPZ Research Befragung der Projektleiterinnen und -leiter von finanzierten Projekten der HRSM-Ausschreibung 2016, $\mathrm{n}=27$

\subsubsection{Vergabeentscheidung}

Die Transparenz des Vergabeverfahrens bewerteten $41 \%$ der Projektleiterinnen und -leiter als (sehr) hoch, $33 \%$ als mittel und $15 \%$ als niedrig. Die Angemessenheit der Dauer des Auswahlverfahrens wurde ähnlich eingeschätzt. $39 \%$ bewerteten sie als (sehr) hoch, $33 \%$ als mittel und $19 \%$ als niedrig. Die Nachvollziehbarkeit der Vergabeentscheidung wurde von $34 \%$ als (sehr) hoch, von $48 \%$ als mittel und von $7 \%$ als niedrig angesehen.

Im Vergleich zur HRSM-Ausschreibung 2013 fiel das Urteil der Befragten etwas besser aus, insbesondere auch deshalb, weil die Antwortmöglichkeit „sehr niedrig“ kein einziges Mal angegeben wurde. Dennoch scheint es so, dass es noch Verbesserungspotential gibt. Was die Kommission selbst anbelangt, so blieb diese gegenüber der HRSM-Ausschreibung 2013 bestehen. So waren bei der HRSM-Ausschreibung 2016 neben zwei Vertretern des BMBWF, ein Vertreter der uniko und ein Vertreter des BMF in der Auswahlkommission präsent. Anders als bei der HRSM-Ausschreibung 2013 war - ergänzend zur Beratung der Kommission - die Einbindung des Qualitätssicherungsrates für Pädagoginnen- und Pädagogenbildung ${ }^{22}$ sowie die Einholung weiterer externer Expertise (wie z.B. durch das Institut für Höhere Studien) gegeben. Außerdem wurde, laut Angabe des BMBWF, im Rahmen der Ausschreibung 2016 auch abgelehnten Projekten Feedback über die Gründe der Ablehnung kommuniziert. Gegenüber der Ausschreibung von 2013 wurden damit bereits erste Änderungen vollzogen.

\section{Abbildung 57: Vergabeverfahren}

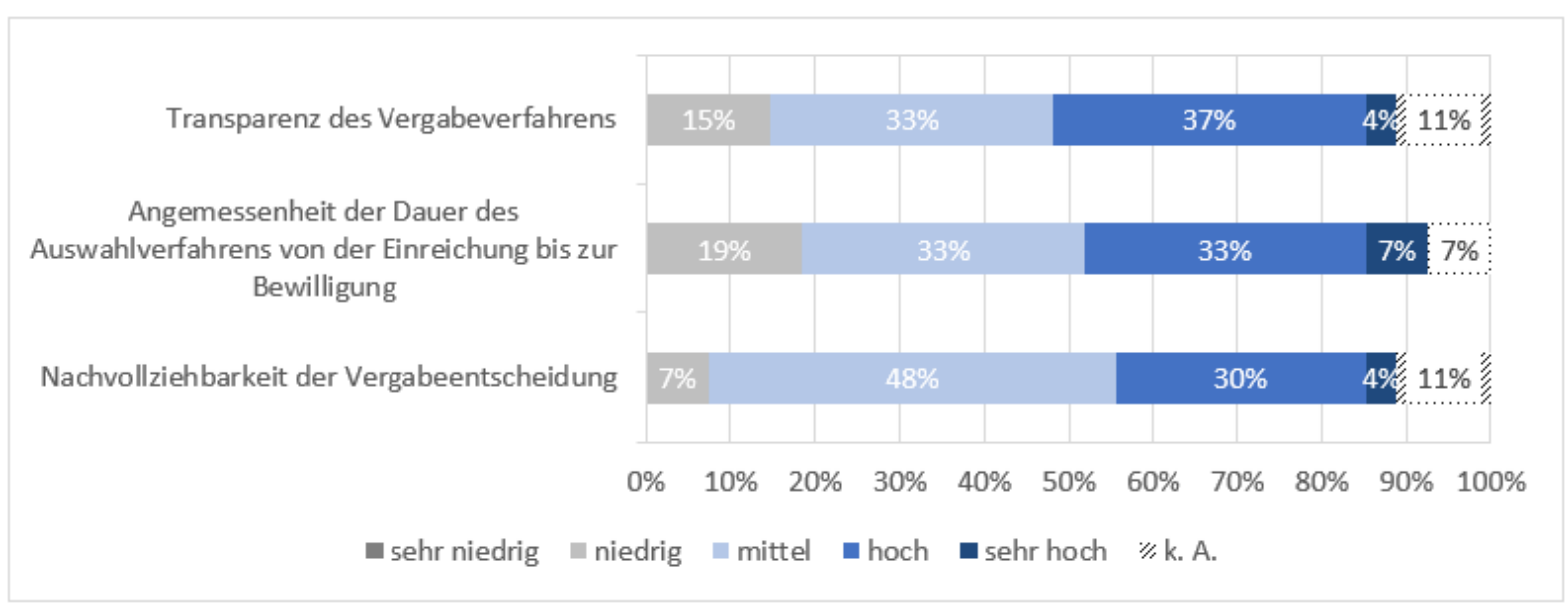

Quelle: WPZ Research Befragung der Projektleiterinnen und -leiter von finanzierten Projekten der HRSM-Ausschreibung 2016, $\mathrm{n}=27$

22 Vgl. https://www.qsr.or.at 
Von den 48 Projektanträgen wurden schließlich 16 abgelehnt, davon sieben im Bereich PädagogInnenbildung Neu und neun im Bereich Sonstige Lehrprojekte (siehe Abbildung 58). Im Vergleich zur HRSM-Ausschreibung 2013 wurden fast doppelt so viele Projekte (vgl. 17 Projekte) im Bereich Lehre finanziert. Es entfielen rund $85 \%$ der Mittel an den Bereich PädagogInnenbildung Neu, während nur knapp über 55 \% der Projekte diesem Bereich zugeordnet werden können. Dies lässt sich durch die besondere Schwerpunktsetzung der Ausschreibung sowie durch die Art, inhaltliche Ausrichtung und Größe der Projekte im Bereich PädagogInnenbildung Neu erklären. Während Projekte im Bereich Sonstige Lehrprojekte meist einzelne Initiativen zu bestimmten Themen (wie z.B. Machbarkeitsstudien, Digitalisierung in der Lehre durch E-Learning Maßnahmen, Mentoring etc.) darstellten, dienten jene im Bereich PädagogInnenbildung Neu der inhaltlichen, personellen, administrativen und technischen Entwicklung sowie der Umsetzung eines gemeinsamen Studienangebots im Entwicklungsverbund.

\section{Abbildung 58: Verhältnis zwischen finanzierten und nicht-finanzierten Projekten (in absoluten Zahlen)}

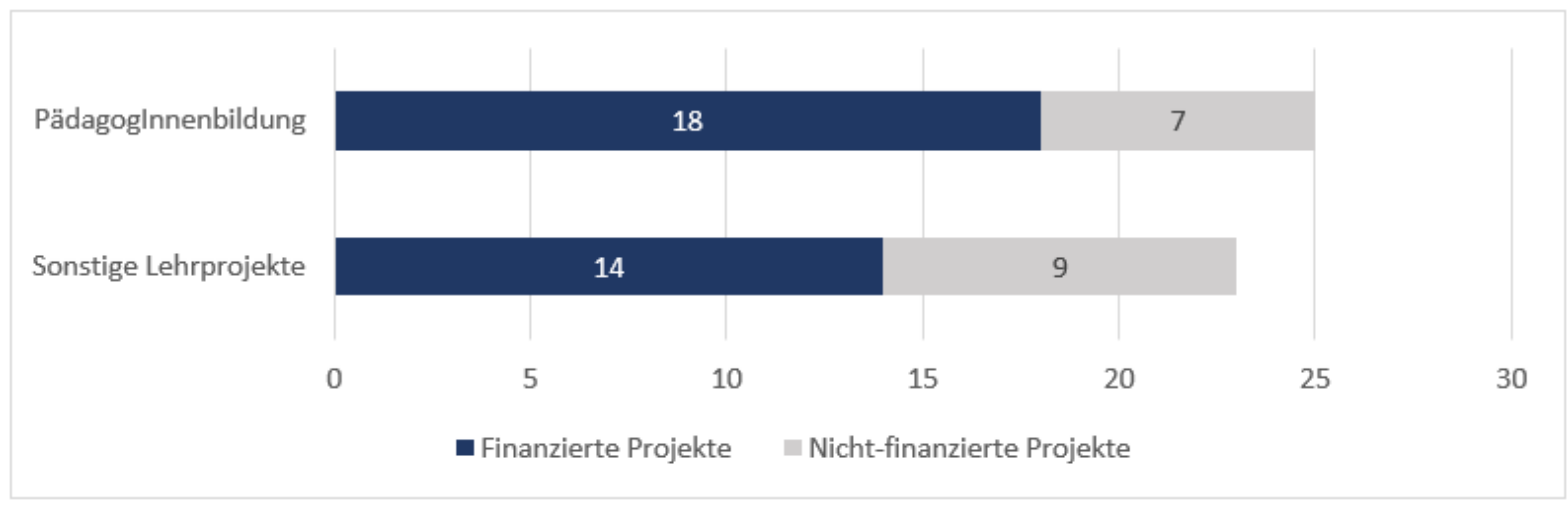

Quelle: BMBWF, eigene Berechnungen; $n=48$

Die Bewilligungsquote der HRSM-Ausschreibung 2016 liegt insgesamt bei 72 \% im Bereich PädagogInnenbildung Neu bzw. 61 \% im Bereich Sonstige Lehrprojekte (siehe Abbildung 59). Verglichen mit der HRSM-Ausschreibung 2013 im Bereich Lehre ist die durchschnittliche Bewilligungsquote beider Bereiche von rund $67 \%$ damit in der Ausschreibung 2016 erheblich höher (vgl. 38 \% in 2013). Dies mag auf die Schwerpunktsetzung sowie die höhere Dotierung des Bereichs Lehre in der jüngeren Ausschreibung zurückzuführen sein.

Generell ist die Bewilligungsquote für den Bereich PädagogInnenbildung Neu deutlich höher und entspricht der Erwartung, dass die HRSM-Ausschreibung 2016 vornehmlich der Entwicklung und Umsetzung dieses bildungspolitischen Ziels dienen sollte. Der Schwerpunkt ist auch über die Universitätstypen hinweg erkennbar. Bei jenen Typen, also Volluniversitäten und Kunstuniversitäten, die in beiden Bereichen aktiv waren, liegt die Bewilligungsquote für Projekte im Bereich Sonstige Lehrprojekte erheblich unter jener im Bereich PädagogInnenbildung Neu. Eine Ausnahme stellt die Gruppe der Sonstigen Universitäten dar.

Die höchste Bewilligungsquote (100\%) lässt sich bei den Technischen Universitäten im Bereich Sonstige Lehrprojekte sowie bei den Sonstigen Universitäten in beiden Bereichen finden. Jedoch ist auf die sehr niedrige Gesamtzahl hinzuweisen. Technische Universitäten haben lediglich drei Projekte beantragt, Sonstige Universitäten nur drei Projekte im Bereich PädagogInnenbildung Neu sowie vier Projekte im Bereich Sonstige Lehrprojekte. Im Kontext der Anzahl der Beantragungen der Sonstigen Universitäten ist außerdem darauf hinzuweisen, dass lediglich drei Universitäten (Universität Linz, Universität Klagenfurt, Universität für Bodenkultur) Lehramtstudien anbieten und somit Anlass für eine Einreichung im Bereich PädagogInnenbildung Neu hatten und diese auch wahrgenommen haben. Im Vergleich weist die aktivste Gruppe der Volluniversitäten (22 Anträge) eine niedrigere Bewilligungsquote von $79 \%$ im Bereich PädagogInnenbildung Neu und $50 \%$ im Bereich Sonstige Lehrprojekte auf. Kunstuniversitäten erzielen im Durchschnitt die niedrigste Bewilligungsquote: 50 \% im Bereich PädagogInnenbildung Neu sowie 20 \% im Bereich Sonstige Lehrprojekte. Jedoch ist insbesondere im Bereich Sonstige Lehrprojekte auf die niedrige Gesamtzahl von fünf Anträgen hinzuweisen. 
Abbildung 59: Bewilligungsquoten nach Universitätstyp und Bereich, sowie Durchschnitt

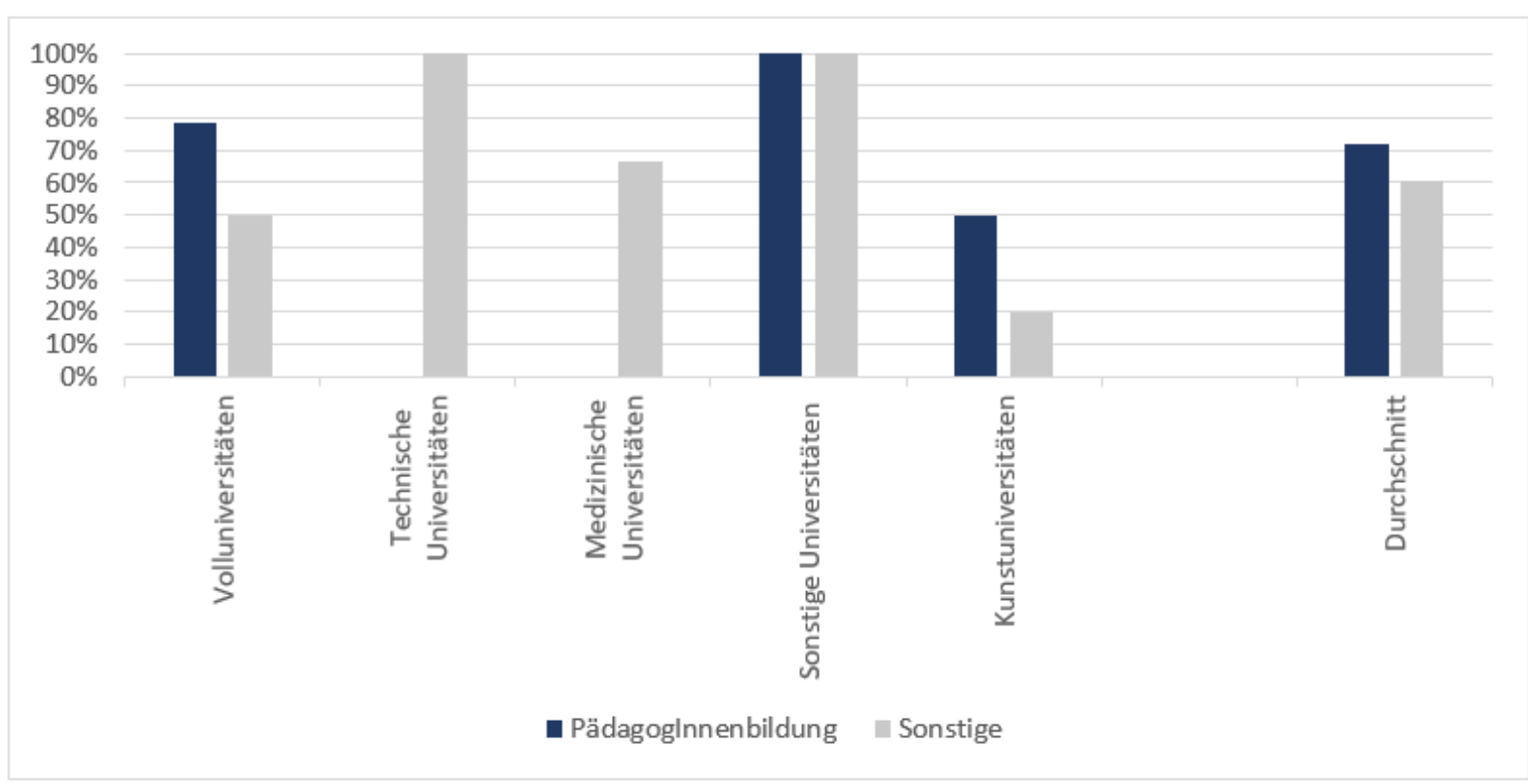

Quelle: BMBWF, eigene Berechnungen; $n=48$

Allgemein lässt sich erkennen, dass Universitäten recht selektiv in der Antragstellung waren. So haben die Universität für Bodenkultur Wien, die Universität Linz, die Universität für Musik und darstellende Kunst Wien und die Universität Mozarteum Salzburg nur Projekte im Bereich PädagogInnenbildung Neu beantragt bzw. gewonnen und keine Antragstellung im Bereich Sonstige Lehrprojekte vorgenommen. Die Technischen und Medizinischen Universitäten führen keine Lehramtsstudien und haben gemäß den Zielen der PädagogInnenbildung Neu keine Anträge in diesem Bereich gestellt und nur Projekte im Bereich Sonstige Lehrprojekte beantragt. Insgesamt waren vier teilnahmeberechtigte Universitäten (Medizinische Universität Innsbruck, Montanuniversität Leoben, Veterinärmedizinische Universität Wien, Universität für Musik und darstellende Kunst Graz) in der HRSM-Ausschreibung 2016 gar nicht als projektleitende Universität aktiv, während sechs Universitäten (Technische Universität Wien, Medizinische Universität Wien, Universität für Bodenkultur Wien, Universität Linz, Universität für angewandte Kunst Wien, Universität für Musik und darstellende Kunst Wien) nur jeweils ein Projekt beantragt haben.

Im Bereich PädagogInnenbildung Neu lassen sich die meisten Anträge sowie finanzierten Projekte auf die Volluniversitäten der jeweiligen Entwicklungsverbünde zurückführen. So haben die Universität Wien (Entwicklungsverbund Nord-Ost), die Universität Innsbruck (Entwicklungsverbund West) und die Universität Salzburg (Entwicklungsverbund Mitte) die meisten Projekte (jeweils drei) eingeworben, gefolgt von der Universität Graz (Entwicklungsverbund Süd-Ost) und der Universität für künstlerische und industrielle Gestaltung Linz (Entwicklungsverbund Mitte) mit jeweils zwei Projekten. Die Analyse der beantragten bzw. bewilligten Finanzierungssummen (siehe Kapitel 3.2.2) bestätigt die Relevanz der Volluniversitäten. Die Universitäten Wien, Graz, Innsbruck und Salzburg beantragten bzw. erhielten auch die höchsten Finanzierungssummen. Hingegen wurde keines der drei beantragten Projekte im Bereich PädagogInnenbildung Neu unter der Leitung der Universität Mozarteum Salzburg finanziert. Im Bereich Sonstige Lehrprojekte ist das Bild wesentlich diverser: Keine Universität leitete mehr als zwei Projekte und auch die Verteilung auf Universitätstypen zeigt sich gleichmäßiger. Die Universität Wien, die Technische Universität Graz, die Medizinische Universität Graz, die Universität Klagenfurt und die Wirtschaftsuniversität Wien konnten jeweils zwei Projekte akquirieren. Im Gegensatz hierzu wurden in diesem Bereich keine Projekte unter der Leitung der Universität Salzburg (zwei Anträge), der Medizinischen Universität Wien (ein Antrag), der Universität für angewandte Kunst Wien (ein Antrag) und der Universität für künstlerische und industrielle Gestaltung Linz (zwei Anträge) finanziert. 


\section{Abbildung 60: Anzahl der finanzierten und abgelehnten Projekte nach Universität und Bereich (in absoluten Zahlen)}

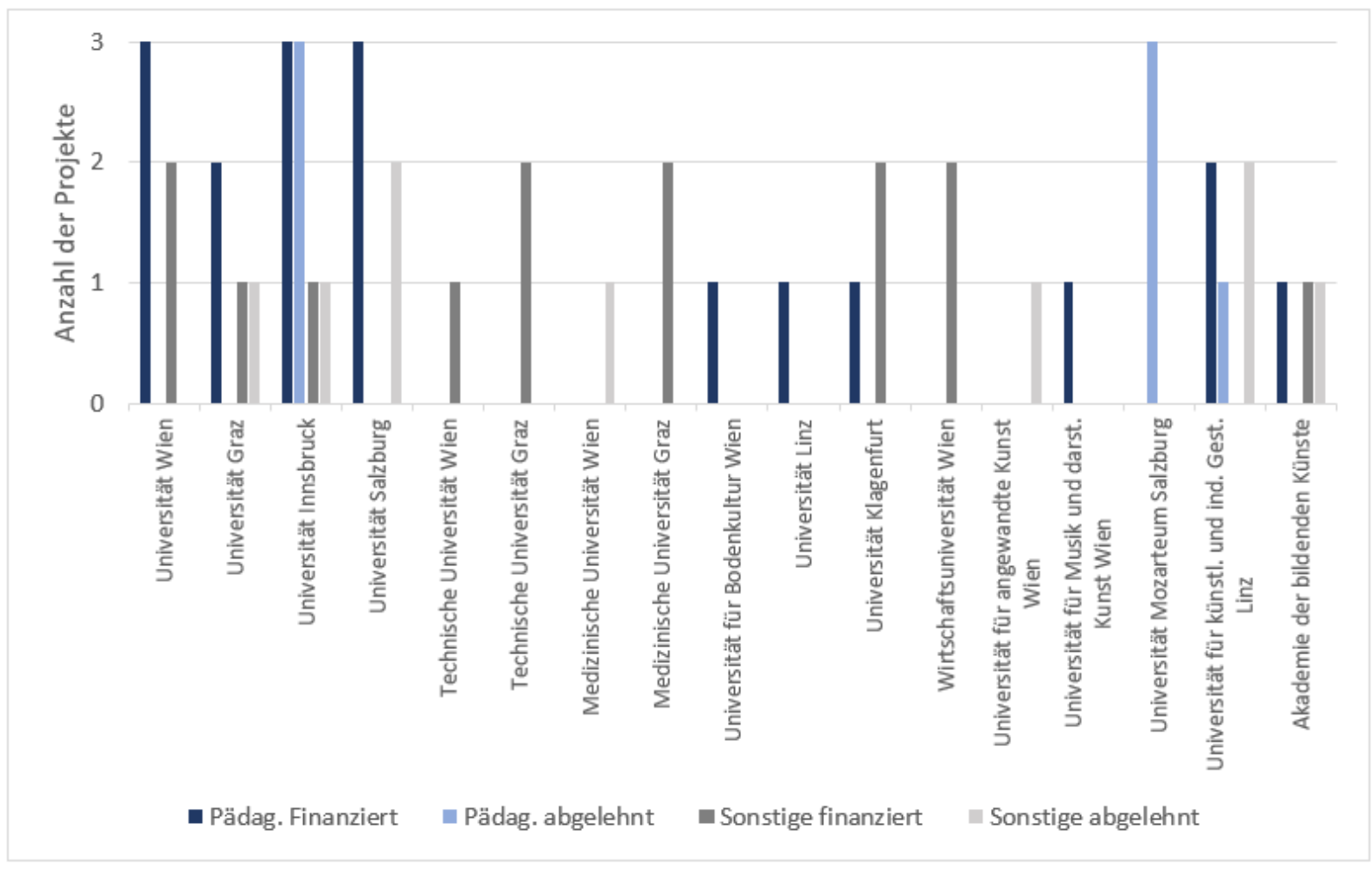

Quelle: BMBWF, eigene Berechnungen; $n=48$

\subsection{Zur Umsetzung}

In Folge wird die Phase der Projektumsetzung näher analysiert. Es stehen hierbei Projektcharakteristika, die Finanzierung, thematische Schwerpunkte wie auch das Kooperationsverhalten der jeweiligen Akteure im Mittelpunkt der Betrachtung.

\subsubsection{Finanzierte Projekte und ihre Charakteristika}

Insgesamt wurden mit der HRSM-Ausschreibung 201632 Projekte finanziert, davon 18 Projekte im Bereich PädagogInnenbildung Neu (56\%) und 14 Projekte im Bereich Sonstige Lehrprojekte (44\%), wobei einige der Sonstigen Lehrprojekte in einem thematischen Zusammenhang mit der PädagogInnenbildung Neu standen. Im Bereich Lehre konnten Volluniversitäten vergleichsweise die höchste Anzahl an Projekten (insgesamt 15 Projekte) lukrieren, davon $73 \%$ im Bereich PädagogInnenbildung Neu (siehe Abbildung 61). Ein ähnliches Verhältnis zeigt sich bei den Kunstuniversitäten, wo 80 \% der Projekte im Bereich Pädagoglnnenbildung Neu durchgeführt wurden, jedoch ist die Gesamtanzahl der bewilligten Projekte hier weitaus niedriger. Bei den Sonstigen Universitäten ist das Verhältnis zwischen den Bereichen PädagogInnenbildung Neu und Sonstige Lehrprojekte 43 \% zu 57 \%. Technische und Medizinische Universitäten haben sich im Vergleich offenbar weniger aktiv an der Ausschreibung beteiligt und wenn, dann zu 100 \% im Bereich Sonstige Lehrprojekte. 


\section{Abbildung 61: Finanzierte Projekte nach Universitätstyp und Bereich (in Prozent)}

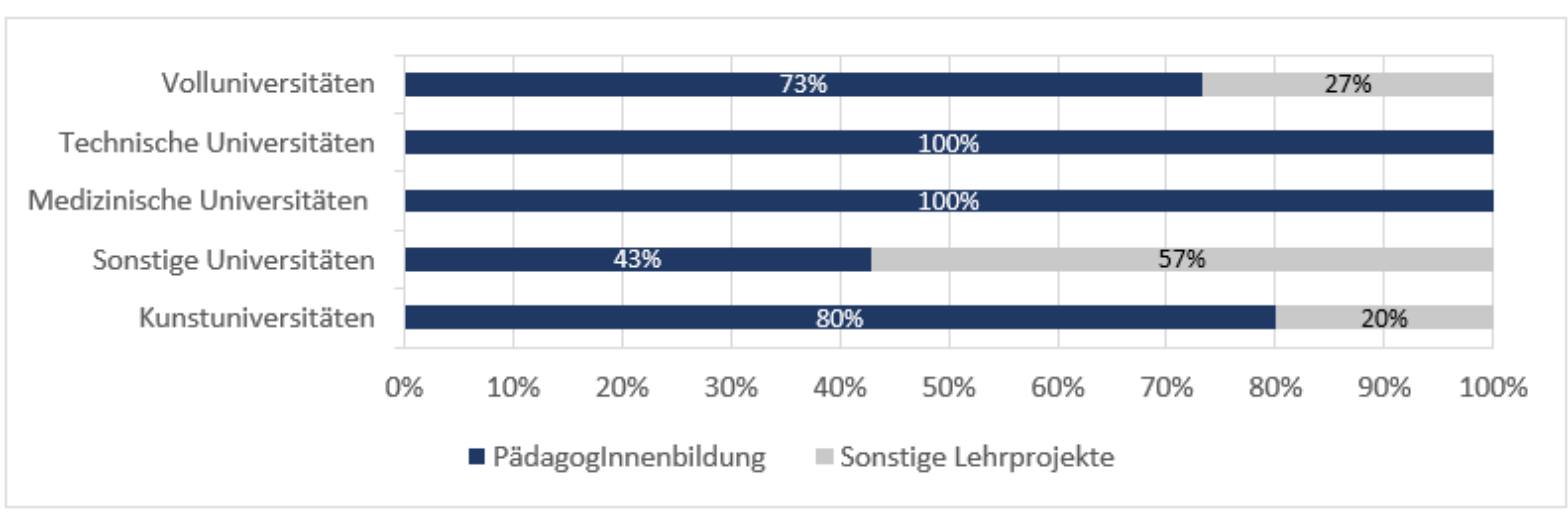

Quelle: BMBWF, eigene Berechnungen; $n=32$

Insgesamt entfällt rund die Hälfte der Projekte (insgesamt 15) auf die Gruppe der Volluniversitäten, gefolgt von den Sonstigen Universitäten mit sieben Projekten und den Kunstuniversitäten mit fünf Projekten (siehe Abbildung 62). Die Universität Wien hat mit fünf Projekten (drei im Bereich PädagogInnenbildung Neu sowie zwei Projekte im Bereich Sonstige Lehrprojekte) die meisten finanzierten Projekte und ist gleichzeitig eine der „erfolgreichsten“ Universitäten in der HRSM-Ausschreibung 2016 mit einer Bewilligungsquote von $100 \%$ gemessen an der Anzahl der bewilligten vs. abgelehnten Projekte. Nach Anzahl der finanzierten Projekte folgen die Universität Innsbruck und die Universität Salzburg (jeweils insgesamt vier Projekte), sowie die Universitäten Graz und Klagenfurt (jeweils insgesamt drei Projekte). Am anderen Ende des Spektrums lassen sich die Technische Universität Wien, die Universität für Bodenkultur Wien, die Universität Linz und die Universität für Musik und darstellende Kunst Wien mit jeweils einem finanzierten Projekt verorten.

Abbildung 62: Anzahl der finanzierten Projekte je Universität und Bereich, sowie nach Universitätstyp (in absoluten Zahlen)

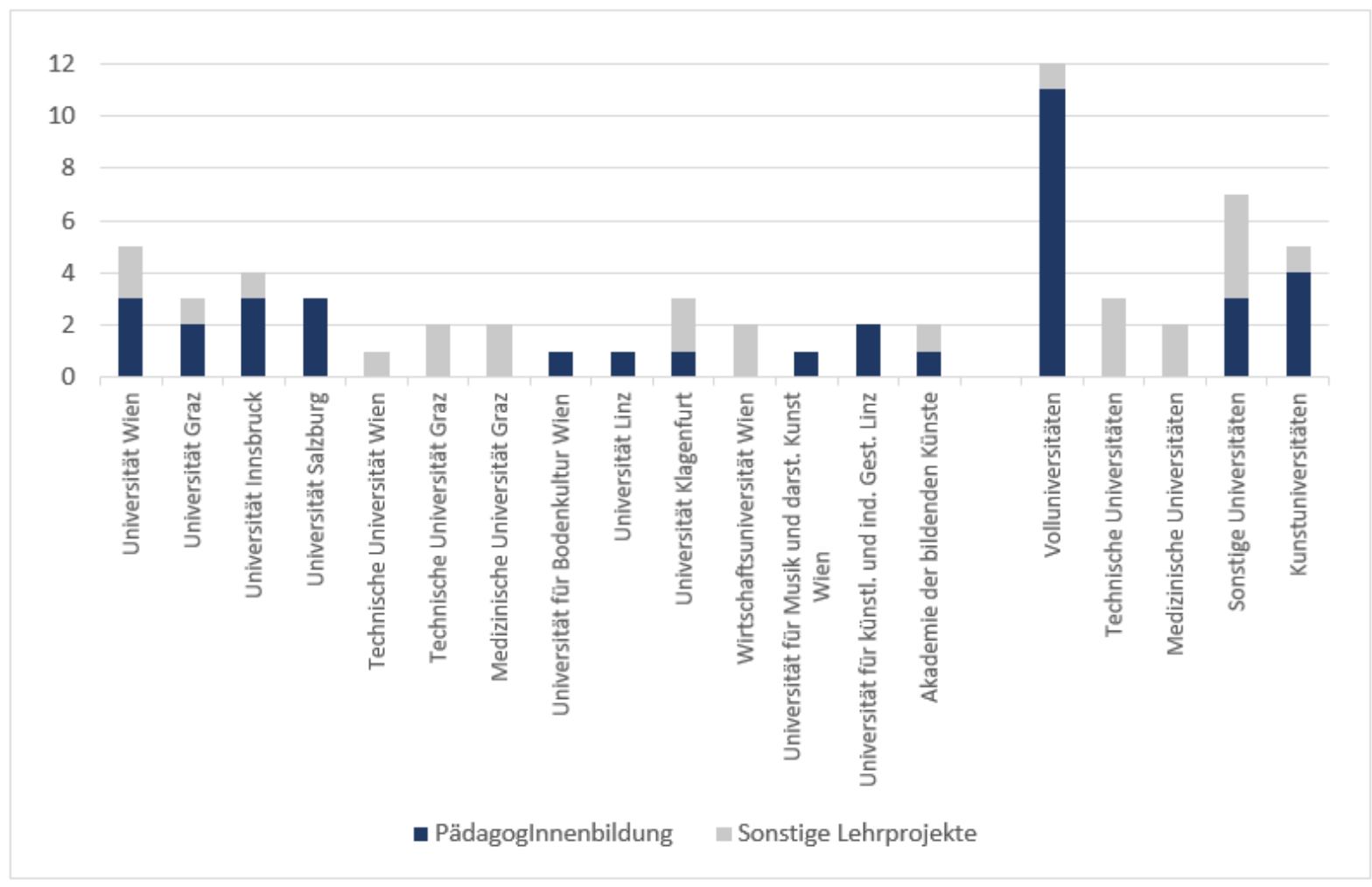

Quelle: BMBWF, eigene Berechnungen; $n=32$ 
Nach Anzahl der Anträge war die Universität Innsbruck mit vier finanzierten Projekten sowie vier nichtfinanzierten Projekten (siehe Abbildung 60) am aktivsten an der HRSM-Ausschreibung 2016 beteiligt, gefolgt von der Universität Wien (fünf finanzierte Projekte), der Universität Salzburg (drei finanzierte, zwei nicht-finanzierte Projekte), und der Universität für künstlerische und industrielle Gestaltung Linz (zwei finanzierte, drei nichtfinanzierte Projekte). Bei der Universität Mozarteum Salzburg (insgesamt drei Anträge), der Medizinischen Universität Wien (insgesamt ein Antrag) und der Universität für angewandte Kunst Wien (insgesamt ein Antrag) wurde kein Projekt finanziert.

\subsubsection{Finanzierung}

Im Gegensatz zur HRSM-Ausschreibung 2013 wurde die Regelung zur Maximalfinanzierung von 1/3 der Projektkosten für die HRSM-Ausschreibung 2016 gelockert: Projekte konnten - in sachlich begründeten Fällen via einstimmige Empfehlung der Auswahlkommission - mehr als 1/3 der Gesamtkosten als BMBWF-Finanzierung beantragen (BMBWF, 2016). Insgesamt wurden 57,76 Mio. Euro beantragt, die Projektkosten (bei Projektende) lagen bei 275,88 Mio. Euro. Die durchschnittlich beantragte Finanzierungssumme lag bei $38 \%$ der Projektkosten (bei Projektende). Die Vergabesumme des BMBW lag bei 35,3 Mio. Euro. In rund 56 \% der Fälle (18 Projekte) lag die Vergabesumme unter der beantragten Finanzierung.

Auf den Bereich PädagogInnenbildung Neu entfielen 29,8 Mio. Euro an Finanzierung und es wurden hier 51,6 Mio. Euro bei Projektkosten von 213,8 Mio. Euro beantragt (siehe Abbildung 63). Bei 11 (61\%) der Projekte in diesem Bereich lag die Vergabesumme unter der beantragten Finanzierung. Im Bereich Sonstige Lehrprojekte wurden Projekte in der Höhe von 5,5 Mio. Euro finanziert; dabei wurden 6,1 Mio. Euro bei Projektkosten von 14,6 Mio. Euro beantragt. Demnach lag die Finanzierung in 50 \% der Fälle (bei 7 Projekten) in diesem Bereich unter der beantragten Summe.

Abbildung 63: Projektkosten (Projektende), beantragte und Vergabesumme (BMBWF) nach Bereichen (in Mio. Euro)

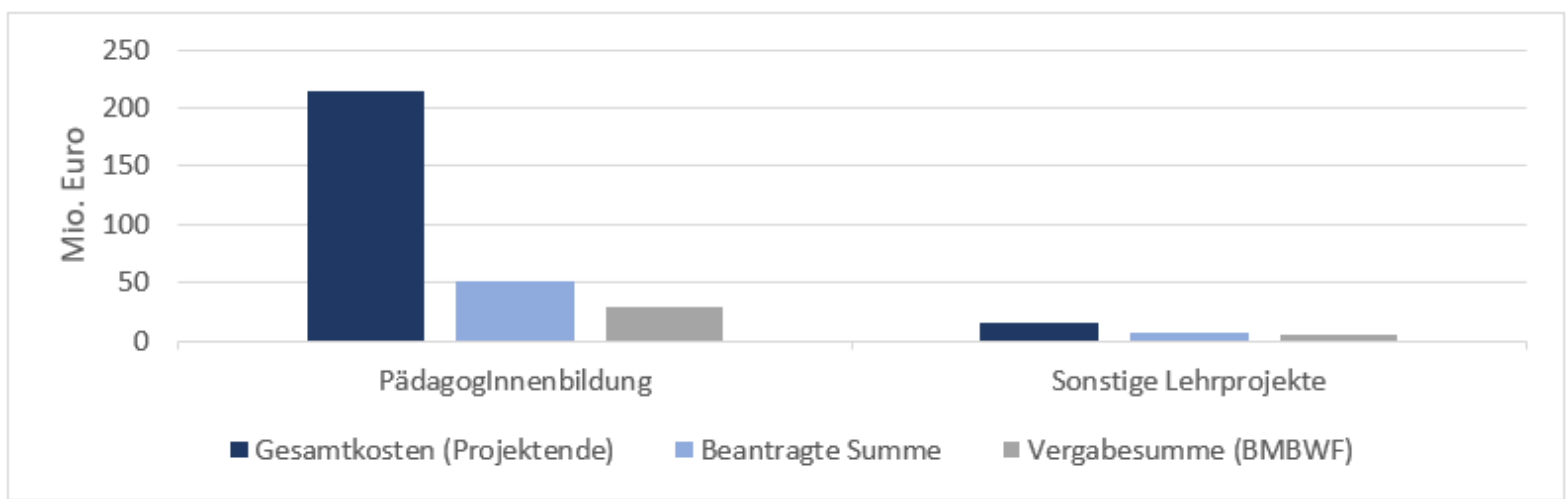

Quelle: BMBWF, eigene Berechnungen; $n=32$

Auf Volluniversitäten entfiel der größte Anteil (knapp 70 \%, 24,6 Mio. Euro) der Finanzierung in der HRSMAusschreibung 2016 für Lehre (siehe Abbildung 64), gefolgt von den Sonstigen Universitäten (6,3 Mio. Euro), Kunstuniversitäten (2,8 Mio. Euro), Medizinischen Universitäten (0,85 Mio. Euro) und Technischen Universitäten (0,78 Mio. Euro). Die Volluniversitäten wiesen auch die höchsten Projektkosten (bei Projektende) mit insgesamt 234,9 Mio. Euro auf, gefolgt von den Sonstigen Universitäten (31,6 Mio. Euro), Kunstuniversitäten (4,7 Mio. Euro), Medizinischen Universitäten (2,37 Mio. Euro) und Technischen Universitäten (2,36 Mio. Euro). 
Abbildung 64: Projektkosten (Projektende), beantragte und Vergabesumme (BMBWF) nach Universitätstyp (in Mio. Euro)

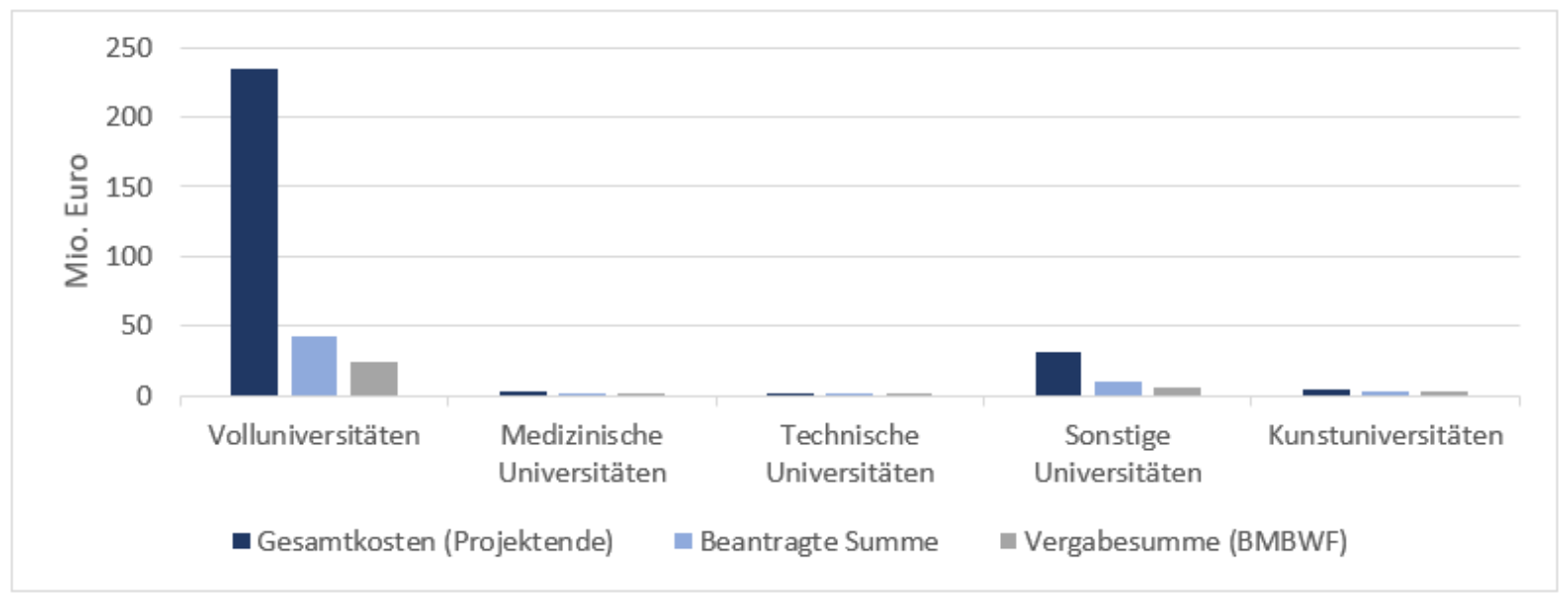

Quelle: BMBWF, eigene Berechnungen; $n=32$

Dieses Bild spiegelt sich natürlich auch in den Projektkosten der einzelnen Universitäten (siehe Abbildung 65) wider. Die Universität Graz verzeichnete mit Abstand die höchsten Projektkosten (104,8 Mio. Euro), gefolgt von der Universität Salzburg (63,3 Mio. Euro), der Universität Wien (53,6 Mio. Euro) und der Universität Innsbruck (13 Mio. Euro). Am anderen Ende des Spektrums zeigt sich die Universität für Bodenkultur Wien mit lediglich einem finanzierten Projekt (bzw. mit Projektkosten in Höhe von 0,45 Mio. Euro).

Abbildung 65: Projektkosten (bei Projektende) nach Universität (in Mio. Euro)

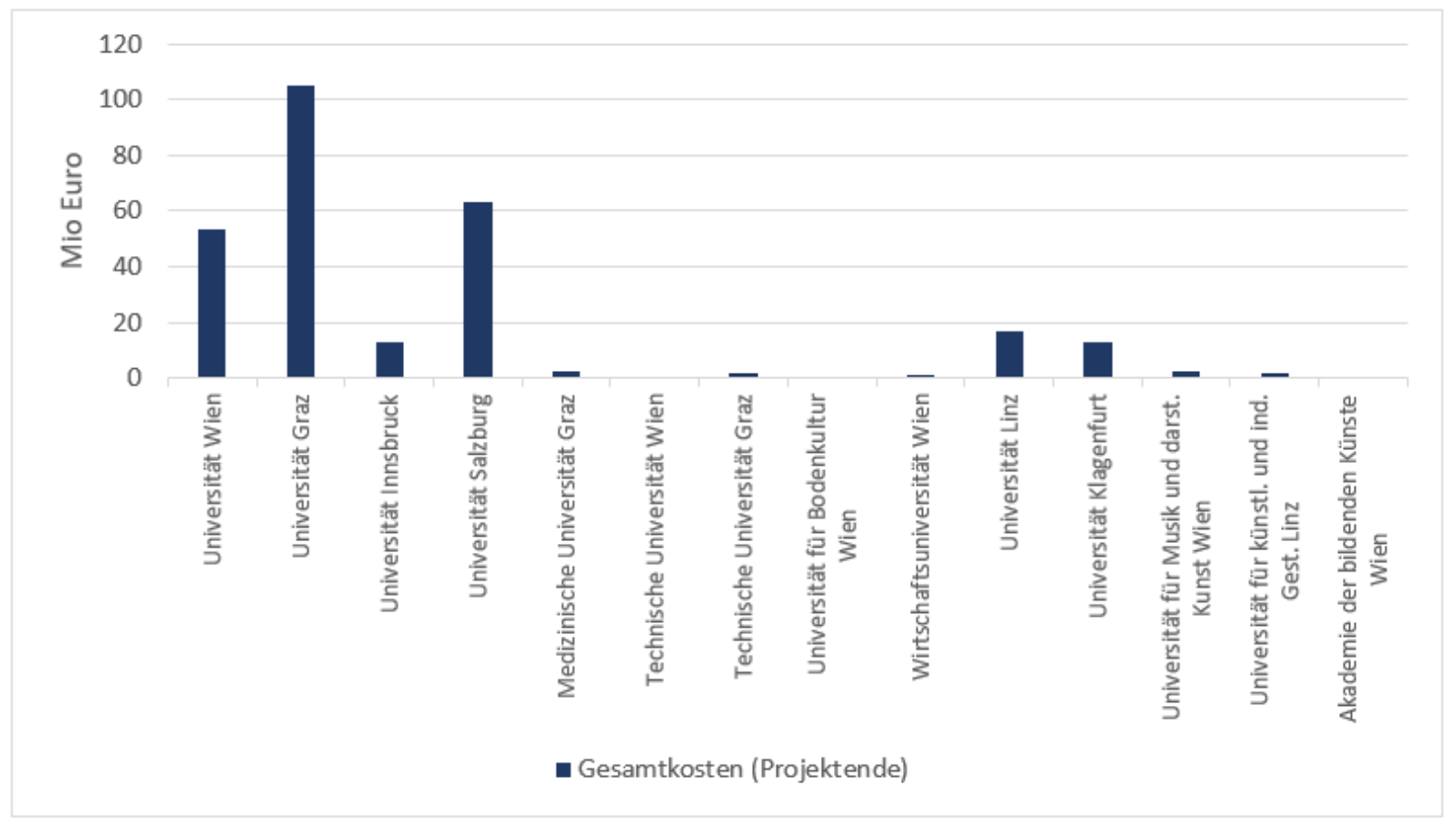

Quelle: BMBWF, eigene Berechnungen; $n=32$

Während die Universität Graz die höchsten Projektkosten aufweist (Abbildung 66) konnte die Universität Wien den höchsten Anteil an HRSM-Mitteln lukrieren (10,4 Mio. Euro), gefolgt von der Universität Graz (6,8 Mio. Euro), der Universität Innsbruck (4,1 Mio. Euro) und der Universität Salzburg (3,4 Mio. Euro). Entsprechend den Projektkosten erhielt die Universität für Bodenkultur Wien auch den kleinsten Anteil der HRSM-Ausschreibung 2016 im Bereich Lehre (0,13 Mio. Euro). 
Gesamthaft betrachtet wird das Gesamtbild der Relevanz der Volluniversitäten bestätigt. So sind diejenigen Universitäten, welche die meisten Projekte leiteten (die Universitäten Wien, Innsbruck, Graz, Salzburg) auch diejenigen, welche die höchsten Finanzierungssummen beantragten bzw. lukrieren konnten.

\section{Abbildung 66: Beantragte und Vergabesumme (BMWF) nach Universität (in Mio. Euro)}

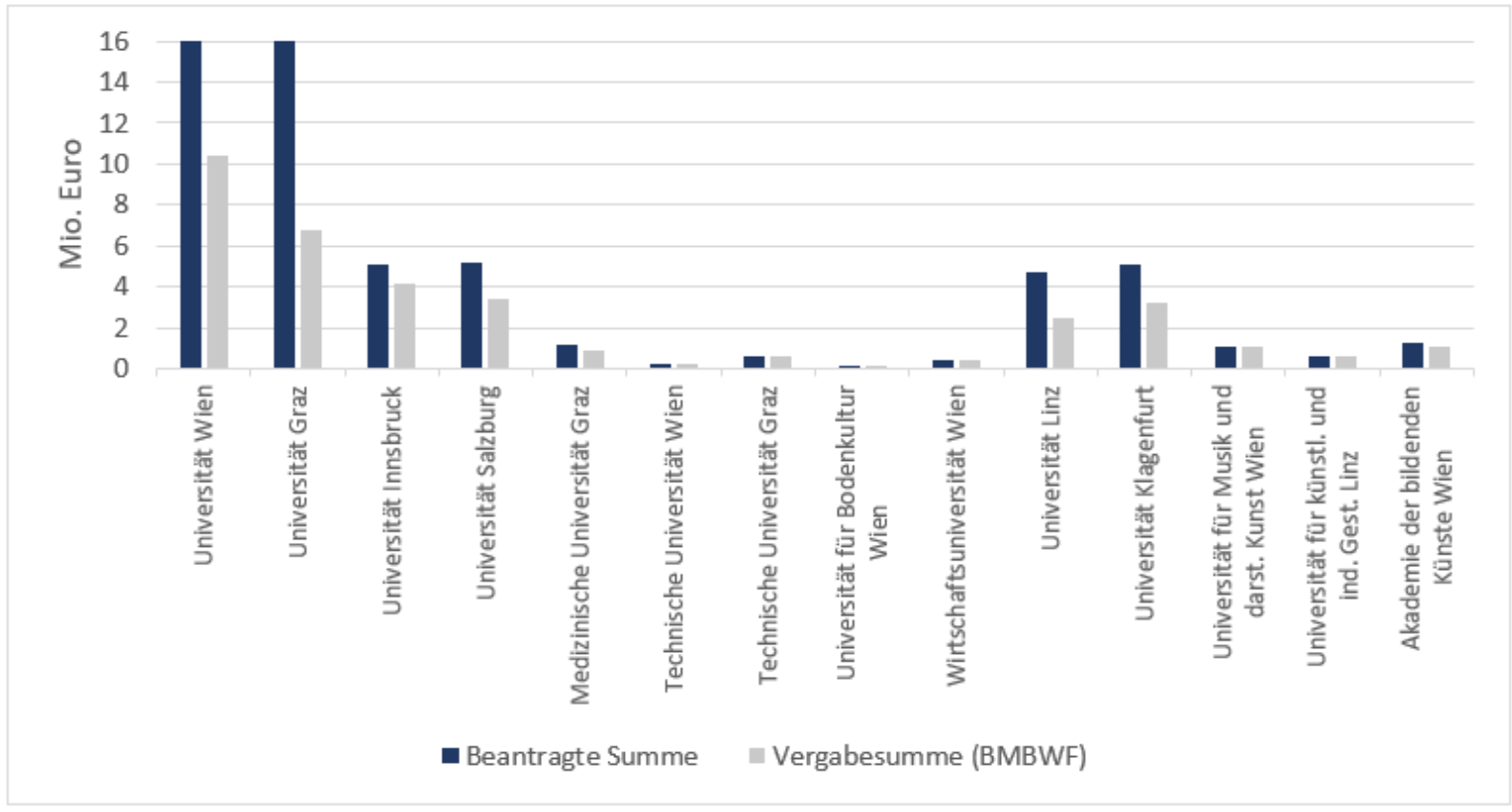

Quelle: BMBWF, eigene Berechnungen; $n=32$

\section{Auf einem Blick:}

Von den 48 beantragten Projekten in der Ausschreibung der Anschubfinanzierung von Kooperationen der Universitäten im Bereich Lehre im Jahr 2016 wurden insgesamt 32 Projekte durch das BMBWF finanziert. Insgesamt wurden 35,3 Mio. Euro vergeben, davon entfielen $84 \%$ (29,8 Mio. Euro) an den Teilbereich PädagogInnenbildung Neu und 16 \% (5,5 Mio. Euro) an den Teilbereich Sonstige Lehrprojekte. Insgesamt lagen die Projektkosten (bei Projektende) bei 275,88 Mio. Euro. Der Anteil der Finanzierung durch das BMBWF betrug insgesamt rund $13 \%$ der Projektkosten.

Die Volluniversitäten konnten durch ihre Größe und das breite Spektrum an verschiedenen Disziplinen und Fachgebieten die meisten Projekte einwerben. So entfiel rund die Hälfte der Projekte (insgesamt 15) auf die Gruppe der Volluniversitäten, gefolgt von den Sonstigen Universitäten mit sieben Projekten und den Kunstuniversitäten mit fünf Projekten (siehe Abbildung 61). Dieses Bild spiegelt sich auch in der Höhe der eingeworbenen Hochschulraumstrukturmittel wider. Auf Volluniversitäten entfiel der größte Anteil (knapp $70 \%$, 24,6 Mio. Euro) der Finanzierung in der HRSM-Ausschreibung 2016 für Lehre (siehe Abbildung 63), gefolgt von den Sonstigen Universitäten (6,3 Mio. Euro), Kunstuniversitäten (2,8 Mio. Euro), Medizinischen Universitäten (0,85 Mio. Euro) und Technischen Universitäten (0,78 Mio. Euro).

Zusammenfassend kann festgehalten werden, dass es durchaus zu Unterschieden in der Höhe der beantragten Mittel und der tatsächlichen Vergabesummen sowie der Projektkosten zwischen den Teilbereichen PädagogInnenbildung Neu und Sonstige Lehrprojekte wie auch zwischen den Universitätstypen gekommen ist. 
Die Finanzierungshöhe bewerteten $42 \%$ der Projektleiterinnen und -leiter im Zuge der Online-Befragung als genau richtig; mit $42 \%$ waren aber ebenso viele der Meinung, dass die Höhe der HRSM-Finanzierung zu niedrig war (siehe Abbildung 67). Zwischen Projekten mit Schwerpunkt auf PädagogInnen Neu und sonstigen Lehrprojekten divergiert diesbezüglich das Antwortverhalten. Leiterinnen und Leiter von sonstigen Lehrprojekten sind eher der Überzeugung, dass die Höhe der Finanzierung genau richtig war.

Anknüpfend an die Frage nach der Finanzierungshöhe waren $31 \%$ aller Projektleiterinnen und -leiter der Meinung, dass diese Art der Finanzierung gar behindernd war, lediglich $27 \%$ sahen sie als anreizfördernd an.

\section{Abbildung 67: Höhe der HRSM-Finanzierung 2016 für Kooperationen im Bereich Lehre}

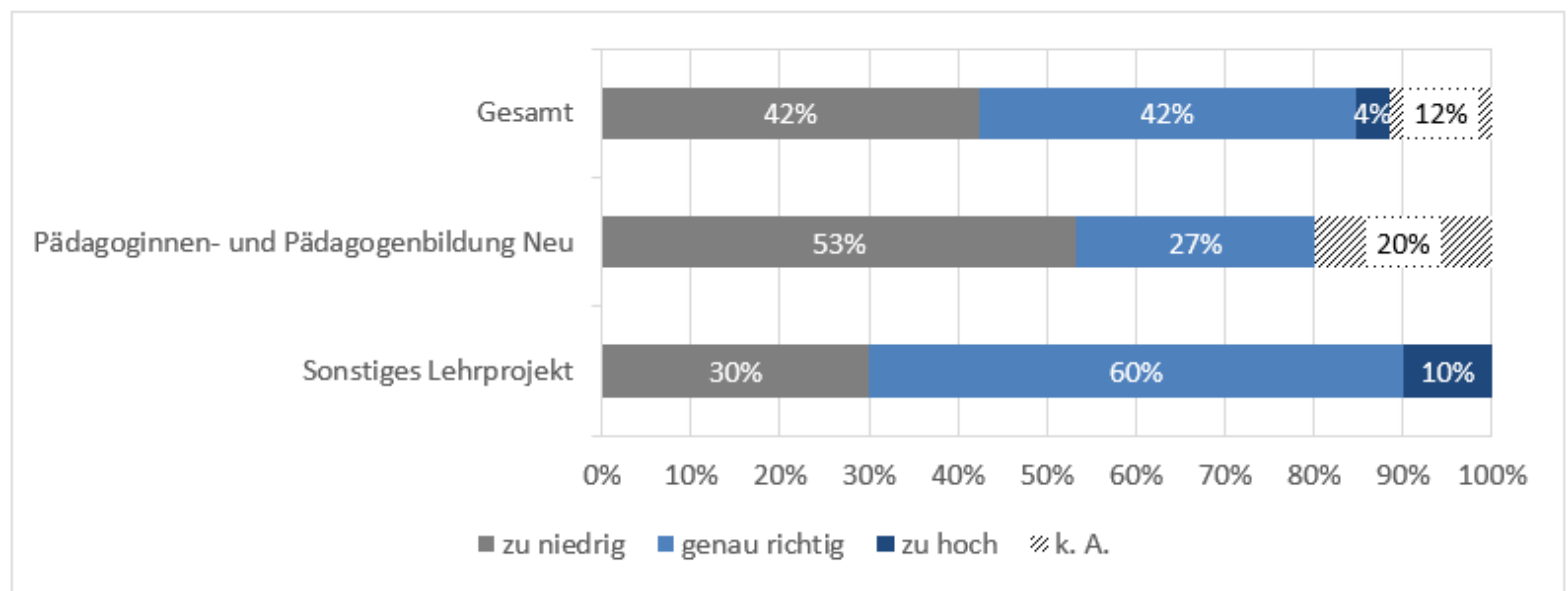

Quelle: WPZ Research Befragung der Projektleiterinnen und -leiter von finanzierten Projekten der HRSM-Ausschreibung 2016, $n=26$

Um künftige Ausschreibungen noch attraktiver zu gestalten, haben befragte Projektleiterinnen und -leiter im Zuge der Online-Befragung Anmerkungen gemacht, wovon eine Auswahl in der folgenden Box dargestellt ist.

\section{Statements der Befragten:}

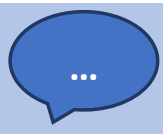

"Klare Regelung für Antragstellung, denn das Anpassen von Kriterien im Nachhinein ist nicht förderlich für das Vertrauen in transparente und objektive Entscheidungen."

„Dass nur 1/3 gefördert wird, ist schwierig, da der Beitrag der Eigenleistung zu einem Großteil aus Personalkosten berechnet wird. Das ist nicht unbedingt zielführend, oder es führt dazu, dass Projekte künstlich "aufgeblasen" werden, damit eine möglichst hohe Fördersumme beantragt werden kann."

"Durch den immer enger werdenden finanziellen Spielraum und die Arbeitslast wissenschaftlicher Mitarbeiterinnen und Mitarbeiter ist ein Eigenanteil von 1/3 bereits sehr schwer realisierbar."

„Volle Kostenübernahme."

„Die hohen inkind-Forderungen sind sehr hinderlich in der Antragsstellung"

Fast alle Projektleiterinnen und -leiter gaben ferner in der Online-Befragung an, dass die weiteren Mittel, die nicht vom BMBWF lukriert werden konnten, aus dem Globalbudget stammten. Drittmittel und sonstige Finanzierungsquellen spielten bei der HRSM-Ausschreibung für Kooperationen offenbar nur eine sehr geringe Rolle. Laut dem BMBWF wurden im Bereich PädagogInnenbildung Neu Finanzierungen der Pädagogischen Hochschulen für ihre Projektbeteiligung geltend gemacht. 


\section{Abbildung 68: Weitere Finanzierungsmittel}

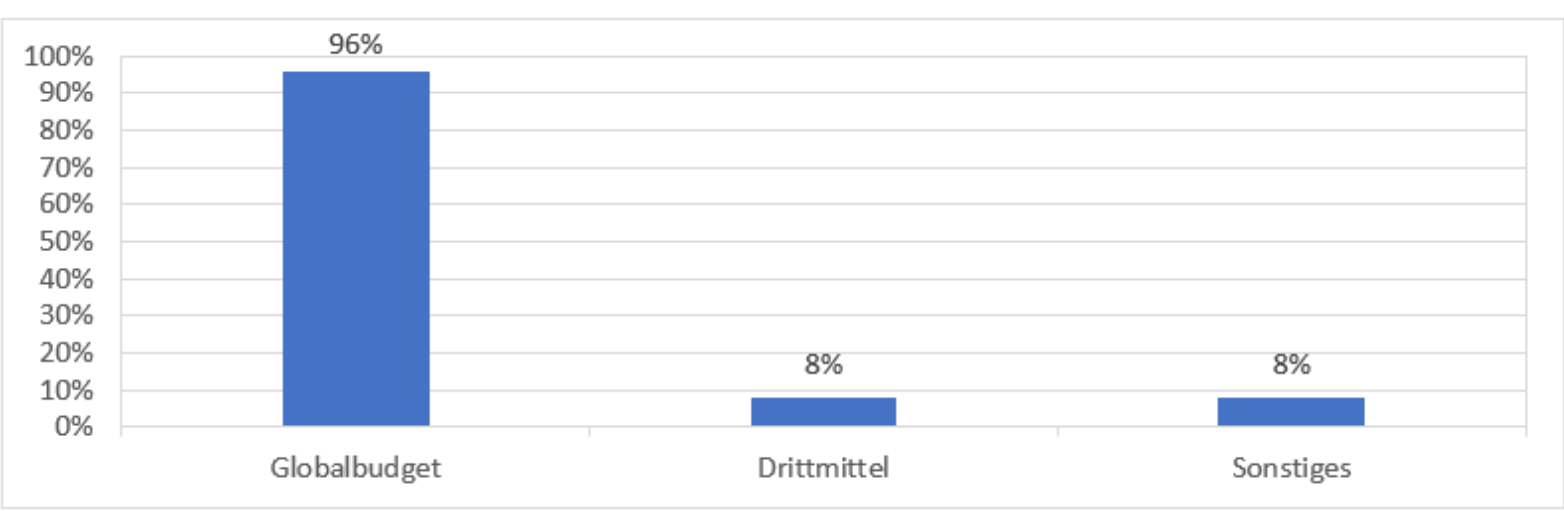

Quelle: WPZ Research Befragung der Projektleiterinnen und -leiter von finanzierten Projekten der HRSM-Ausschreibung 2016, $\mathrm{n}=26$

$58 \%$ der befragten Projektleiterinnen und -leiter gaben an, dass ihr HRSM-Projekt auch Thema bei den Begleitgesprächen zur Leistungsvereinbarung zwischen der Universität und dem BMBWF war. Über ein Drittel (35\%) können allerdings keine Auskunft darüber geben. Das ist vergleichbar mit dem Wert bei der HRSMAusschreibung 2013.

Mit 62 \% bewertete die Mehrzahl der Projektleiterinnen und -leiter die Dauer des Projekt- und Finanzierungszeitraums als genau richtig, 38 \% fanden ihn allerdings zu kurz. Gemäß Analysen der Projektanträge und -berichte betrug der Projekt- und Finanzierungszeitraum im Durchschnitt drei bis vier Jahre. Im Zuge der Befragung wurde ein Zeitraum von fünf bis sechs Jahren besonders häufig gewünscht.

\section{Abbildung 69: Dauer des Projekt- und Finanzierungszeitraums}

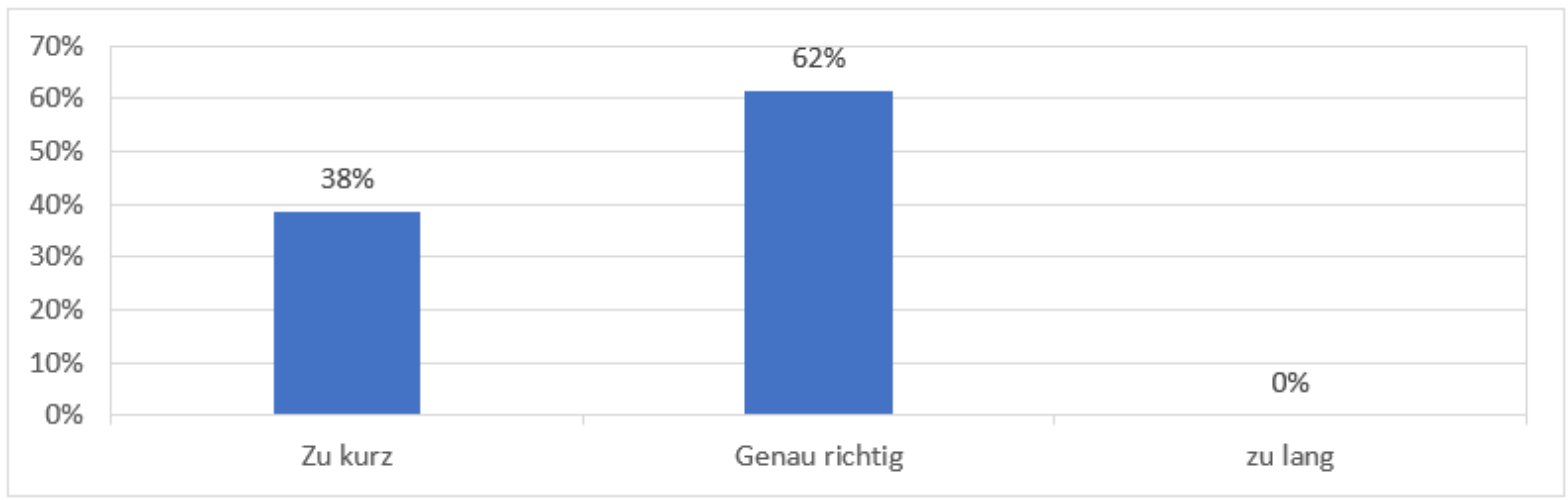

Quelle: WPZ Research Befragung der Projektleiterinnen und -leiter von finanzierten Projekten der HRSM-Ausschreibung 2016, $\mathrm{n}=26$

Den administrativen Aufwand für das Reporting, also das Erstellen von Zwischen- und Endberichten, empfanden knapp zwei Drittel (65 \%) als genau richtig, ein Drittel (33\%) allerdings als zu hoch (siehe Abbildung 70). Letztere Antwortmöglichkeit wurde damit fast doppelt so oft ausgewählt wie bei der HRSM-Ausschreibung 2013. Inhaltlich waren die Projektleiterinnen und Projektleiter vom Reporting eher überzeugt, $28 \%$ empfanden es als (eher) zweckmäßig, $35 \%$ als mittel und $8 \%$ als eher nicht zweckmäßig. Diese Werte sind etwa mit den Werten der HRSM-Ausschreibung 2013 vergleichbar. 
Abbildung 70: Administrativer Aufwand für das Reporting (d.h. für das Verfassen der Zwischenberichte und Endberichte)

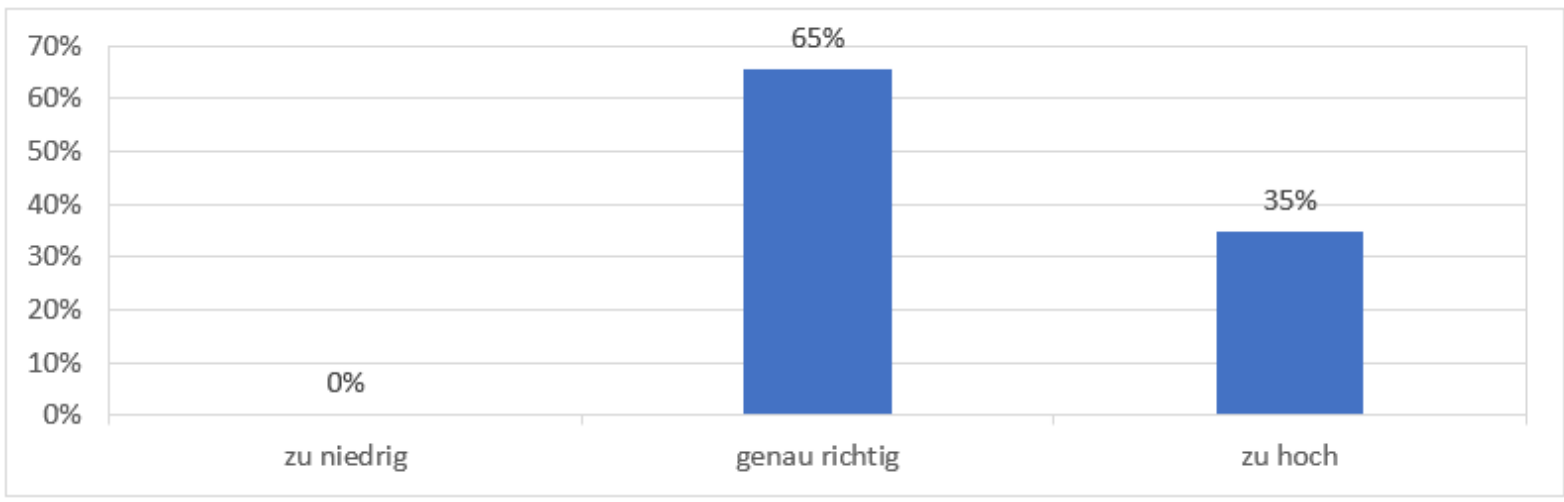

Quelle: WPZ Research Befragung der Projektleiterinnen und -leiter von finanzierten Projekten der HRSM-Ausschreibung 2016, $n=26$

$66 \%$ der Projektleiterinnen und -leiter verorteten eine (sehr) hohe universitätsinterne Unterstützung für ihr Projekt, 31 \% schätzten eine solche Unterstützung als mittel und lediglich $4 \%$ als niedrig ein. Die Unterstützung fiel nach eigenen Angaben damit etwas geringer aus als bei der HRSM-Ausschreibung 2013. Nach Universitätstyp oder thematischem Schwerpunkt sind keine Unterschiede im Antwortverhalten bezüglich universitätsinterner Unterstützung festzustellen.

\section{Abbildung 71: Universitätsinterne Unterstützung für das Projekt}

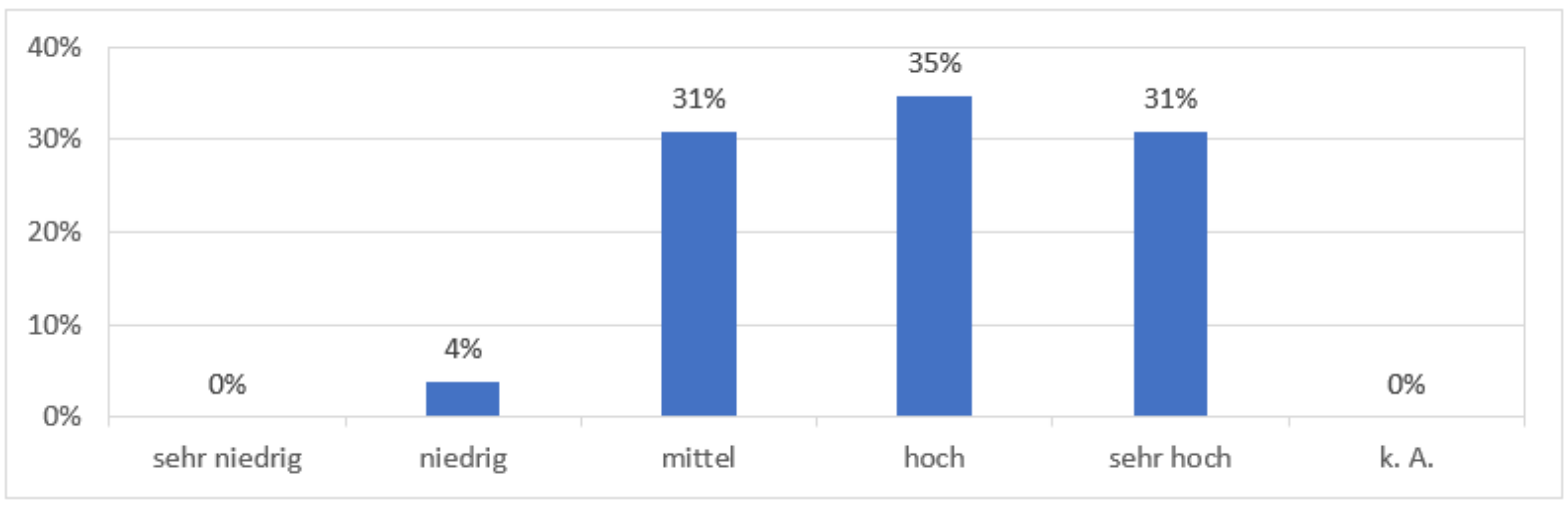

Quelle: WPZ Research Befragung der Projektleiterinnen und -leiter von finanzierten Projekten der HRSM-Ausschreibung 2016, $n=26$

Die Unterstützung kam insbesondere aus vier Bereichen, die jeweils zu rund $30 \%$ genannt wurden: Personal in Service- und Administrationsstellen (35\%), Professorinnen und Professoren (32\%), Rektorat (32\%) und Department-/Institutsleitung (30\%). Damit unterschied sich das Antwortverhalten deutlich von jenem der HRSM-Ausschreibung 2013. Dort hatten das Personal in Service- und Administrationsstellen und das Rektorat Zustimmungswerte von über $75 \%$ zu verzeichnen. Jedoch ist in diesem Kontext auf Unterschiede in der Projektleitung hinzuweisen: Während in der HRSM-Ausschreibung 2013 Wissenschaftlerinnen und Wissenschaftler überwogen, übernahmen in der HRSM-Ausschreibung 2016 zum Großteil die Vize-Rektorinnen und Vize-Rektoren sowie Leiterinnen und Leiter von Service- und Administrationsstellen selbst die Projektleitung. Damit geht natürlich die Unterstützung durch das Rektorat bzw. durch die eigene Service-/Administrationsstelle mit der Projektleitung einher. 
Abbildung 72: Universitätsinterne Unterstützung für das Projekt

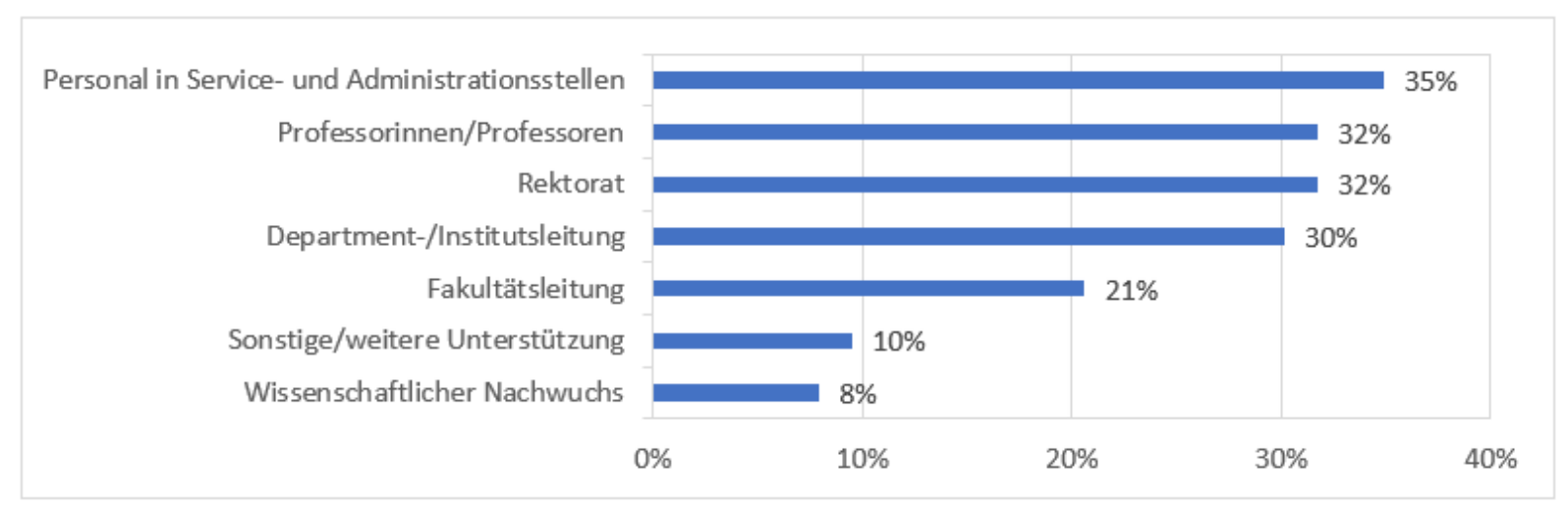

Quelle: WPZ Research Befragung der Projektleiterinnen und -leiter von finanzierten Projekten der HRSM-Ausschreibung 2016, $n=26$

\subsubsection{Thematische Schwerpunkte}

Das prioritäre Ziel der HRSM-Ausschreibung 2016 bestand in der Stärkung von universitären Kooperationsvorhaben im Bereich Lehre wie auch insbesondere in der Unterstützung der Umsetzung der PädagogInnenbildung Neu als zentrales bildungspolitisches Projekt in Österreich (BMBWF, 2016). Demnach sollten nachhaltige Kooperationen zwischen Universitäten wie auch zwischen Universitäten, Hochschulen und Forschungseinrichtungen gestärkt werden. Entsprechend wurden für die HRSM-Mittelvergabe eine Reihe von inhaltlichen Auswahlkriterien (siehe Tabelle 1) definiert, welche zugleich auch die Wirkungsdimensionen der HRSMAusschreibung 2016 wiedergeben. Entsprechend lag im Bereich PädagogInnenbildung Neu der Schwerpunkt neben der Etablierung gemeinsamer Strukturen und der Nutzung von Synergien auf der Sicherung und Weiterentwicklung der Voraussetzungen zur Durchführung gemeinsamer Lehramtsstudien, der Einrichtung und Weiterentwicklung von Lehramtsstudien, der Gewährleistung der Lehrqualität und fachlicher Innovationen sowie der Förderung des wissenschaftlichen Nachwuchses.

Durch den besonderen Schwerpunkt der PädagogInnenbildung Neu sind die beiden Bereiche nur bedingt vergleichbar. Während auch im Bereich Sonstige Lehrprojekte die Etablierung gemeinsamer Strukturen und die Nutzung von Synergien im Fokus standen, adressierten die anderen Kriterien Themen wie universitätsübergreifende Studienangebote, Erhöhung der Studienaktivität und Abschlussquoten, Betreuung nicht-traditionell Studierender, gleiche Teilhabe von Männern und Frauen, Digitalisierung in der Lehre, sowie Erleichterung der innerösterreichischen Mobilität.

Tabelle 1: Inhaltliche Kriterien der Vergabe nach Bereichen

\begin{tabular}{|c|c|}
\hline PädagogInnenbildung Neu & Sonstige Lehrprojekte \\
\hline $\begin{array}{l}\text { I. Etablierung gemeinsamer Strukturen im Bereich des } \\
\text { Lehrens und Lernens für nachhaltige Kooperationen } \\
\text { auch über die konkrete Projektdauer hinaus }\end{array}$ & $\begin{array}{l}\text { 1. Etablierung gemeinsamer Strukturen im Bereich des } \\
\text { Lehrens und Lernens für nachhaltige Kooperationen } \\
\text { auch über die konkrete Projektdauer hinaus }\end{array}$ \\
\hline $\begin{array}{l}\text { II. Sicherung und Weiterentwicklung der wissenschaft- } \\
\text { lichen und professionsorientierten Voraussetzungen } \\
\text { durch die Schaffung kooperativer, insbesondere auch } \\
\text { universitätsübergreifender Arbeitseinheiten in den } \\
\text { Verbundregionen zur Durchführung der Lehramts- } \\
\text { studien unter besonderer Berücksichtigung des zu } \\
\text { erwartenden Bedarfs an Absolventinnen und } \\
\text { Absolventen }\end{array}$ & $\begin{array}{l}\text { 2. Entwicklung universitätsübergreifender Studienan- } \\
\text { gebote unter Nutzung vorhandener Ressourcen und } \\
\text { Synergien }\end{array}$ \\
\hline $\begin{array}{l}\text { III. Einrichtung und Weiterentwicklung von Lehramts- } \\
\text { studien unter Nutzung sämtlicher vorhandener } \\
\text { Ressourcen innerhalb einer Verbundregion und ggf. } \\
\text { über diese hinausgehend unter besonderer Berück- }\end{array}$ & $\begin{array}{l}\text { 3. Konkrete Maßnahmen zur Erhöhung der Studien- } \\
\text { aktivität und der Abschlussquoten }\end{array}$ \\
\hline
\end{tabular}




\begin{tabular}{|c|c|}
\hline PädagogInnenbildung Neu & Sonstige Lehrprojekte \\
\hline \multicolumn{2}{|l|}{$\begin{array}{l}\text { sichtigung des zu erwartenden Bedarfs an Absolvent- } \\
\text { innen und Absolventen }\end{array}$} \\
\hline $\begin{array}{l}\text { IV. Gewährleistung einer qualitätsvollen Lehre in } \\
\text { besonders frequentierten Lehramtsstudien unter } \\
\text { Berücksichtigung des Bedarfs an Absolventinnen und } \\
\text { Absolventen }\end{array}$ & $\begin{array}{l}\text { 4. Institutions- bzw. organisationsübergreifende } \\
\text { Integration und Betreuung nicht-traditioneller } \\
\text { Studierender }\end{array}$ \\
\hline $\begin{array}{l}\text { V. Förderung des wissenschaftlichen Nachwuchses, } \\
\text { insbesondere für die Fachdidaktiken und den } \\
\text { Primarbereich }\end{array}$ & $\begin{array}{l}\text { 5. Förderung der gleichen Teilhabe von Männern und } \\
\text { Frauen an einzelnen Studienfächern (insb. MINT- } \\
\text { Fächern) }\end{array}$ \\
\hline \multirow[t]{2}{*}{$\begin{array}{l}\text { VI. Innovation und fachliche Neuerungen, wie z.B. } \\
\text { fächerübergreifende Angebote zur Förderung von } \\
\text { MINT-Fächern etc. }\end{array}$} & $\begin{array}{l}\text { 6. Innovationen/Optimierungen im Bereich von IT- } \\
\text { basierten Lehr- und Lernformaten }\end{array}$ \\
\hline & $\begin{array}{l}\text { 7. Erleichterung der innerösterreichischen Mobilität } \\
\text { von Studierenden }\end{array}$ \\
\hline
\end{tabular}

Quelle: BMBWF, 2016

Von den Antragstellenden verlangte die HRSM-Ausschreibung 2016 eine qualitative Beschreibung des Projektes in Bezug auf die einzelnen Vergabekriterien. Die Analyse der Projektanträge zeigt folglich, dass die Vergabekriterien durch Projekte im Bereich PädagogInnenbildung Neu insgesamt recht gut erfüllt wurden und diese insgesamt einander auch recht ähnlich waren. Die HRSM-2016-finanzierten Projekte setzten insbesondere am Aus- und Aufbau von Infrastrukturen und Personalressourcen und an der Optimierung von administrativtechnischen Abläufen an, nicht zuletzt um gute/bessere Bedingungen für gemeinsame Lehramtsstudien zu schaffen. Besonders berücksichtigt wurde dabei auch der zu erwartende Bedarf an Absolventinnen und Absolventen in einzelnen Fächern, während Innovation und fachliche Neuerungen in MINT-Fächern (Kriterium VI) meist nur in jenen Projekten, die Personal- bzw. Kapazitätsaufbau in MINT-Fächern zum Ziel hatten, ein Thema waren.

Während die Projektziele bzw. Kriterienerfüllung im Bereich PädagogInnenbildung Neu recht homogen ist, weist der Bereich Sonstige Lehrprojekte ein wesentlich diverseres Projektportfolio auf. Gemäß der Zielsetzung der HRSM-Ausschreibung 2016 kann nach Analyse der Projektanträge geschlossen werden, dass die meisten Projekte das Ziel hatten, gemeinsame Strukturen aufzubauen bzw. zu stärken und vorhandene Synergien zu nutzen. Die inhaltliche Schwerpunktsetzung ist dabei breit gefächert und reicht von unterstützenden Studien und Analysen, Aufbau von E-Learning Formaten, Förderung/Mentoring von Schülerinnen und Schülern und Studierenden bis hin zum Aus- und Aufbau von Infrastrukturen für die Aufnahme in das Studium. Deshalb ist auch die Erfüllung der Kriterien stärker von der inhaltlichen Ausrichtung des Projekts abhängig: So werden nicht in jedem Projekt universitätsübergreifende Studienangebote (Kriterium 2) eingerichtet, auch IT-basierte Lehr- und Lernformate (Kriterium 6) spielen eher in Projekten mit Schwerpunkt auf die Entwicklung und Nutzung der digitalen Lehr-und Lernmöglichkeiten (MOOCs, Microlearning, OER-Ressourcen) eine Rolle. Auch die Erleichterung der innerösterreichischen Mobilität (Kriterium 7) spielt, je nach inhaltlichem Schwerpunkt, in vielen Projekten keine bzw. nur eine kleine Rolle: Durch OER-Ressourcen bzw. niederschwelligen Zugang zu Lerninhalten durch E-Learning wird eine zukünftige Erleichterung der Anerkennung von Studienleistungen erhofft. Eine kleine Anzahl an Projekten, darunter die Projekte „Microlearning Medizinische Propädeutik“ und "Machbarkeitsstudie zur Harmonisierung der humanmedizinischen Ausbildung (Studienjahre 1 - 5) an den öffentlichen Medizinischen Universitäten Österreichs zur Erhöhung der nationalen Mobilität" sollen direkt auf die Erleichterung der Mobilität wirken. Insbesondere ersteres Projekt ist ein Schritt der curricularen Homogenisierung zwischen zwei Universitäten, während die Machbarkeitsstudie die Grundlagen für die Harmonisierung von Lernzielen und Curricula legt. Ebenso lassen sich in diesem Bereich zwei Projekte identifizieren, deren Zielsetzungen bzw. Kriterienerfüllung auf Unterstützung der PädagogInnenbildung Neu abzielen. Dies ist zum einen das Projekt „Verbundinformationssystem - Lehramtsstudien“ unter der Leitung der Universität Klagenfurt, zum anderen das Projekt „Österreichweites gemeinsames Aufnahme- und Auswahlverfahren für Lehramtsstudien“ unter der Leitung der Universität Graz. Diese Gegebenheit ist darauf zurückzuführen, dass beide Projekte sich zwar mit Infrastruktur bzw. administrativen Abläufen der Harmonisierung von Lehramtsstudien befassen, sich jedoch diesen Fragen mit einem österreichweiten Blickwinkel widmen und keinen spezifischen Fokus auf einzelne Entwicklungsverbünde bzw. Studienangebote haben. 


\section{Auf einem Blick:}

Die inhaltlichen Schwerpunkte der Projekte im Teilbereich PädagogInnenbildung Neu und der Projekte im Teilbereich Sonstige Lehrprojekte unterscheiden sich grundsätzlich und sind miteinander kaum vergleichbar. Auch die Konsortiumszusammensetzung unterscheidet sich: Während Kooperationspartnerinnen und -partner für Projekte im Bereich PädagogInnenbildung Neu durch die gemeinsame Teilnahme in einem Entwicklungsverbund entstanden sind, waren Projekte im Bereich Sonstige Lehrprojekte nicht auf diese Entwicklungsverbünde beschränkt. Insgesamt bestätigen die Ergebnisse die Relevanz von vorheriger Zusammenarbeit für die Partnerinnen- bzw. Partnerwahl in der HRSM-Ausschreibung 2016.

Auch zeigen sich Unterschiede in der Anzahl von Kooperationspartnerinnen und -partnern: Im Bereich Sonstige Lehrprojekte wiesen 64 \% der finanzierten Projekte (neun Projekte) ein oder zwei Kooperationspartnerinnen und -partner auf (siehe Abbildung 74). Im Vergleich dazu haben Projekte im Bereich PädagogInnenbildung Neu häufiger mehr Kooperationspartnerinnen und -partner. Lediglich 33 \% der Projekte im Bereich PädagogInnenbildung Neu hatten ein oder zwei Kooperationspartnerinnen bzw. -partner. Die am häufigsten vorkommende Konstellation sind drei Kooperationspartnerinnen und -partner (fünf Projekte bzw. 28 \% der Projekte in diesem Bereich). Weitere 39 \% der Projekte hatten vier, fünf, sechs oder mehr Kooperationspartnerinnen und -partner.

Die inhaltlichen Schwerpunkte der Projekte im Bereich PädagogInnenbildung Neu sind erwartungsgemäß recht homogen: Der Fokus liegt auf dem Auf- und Ausbau von Personal und Infrastruktur für die gemeinsame Umsetzung der Lehramtsstudien im Verbund, inklusive der Schaffung von gemeinsamen Koordinationsstellen und der Optimierung/Harmonisierung von organisatorischen Prozessen.

Die inhaltlichen Schwerpunkte der Projekte im Bereich Sonstige Lehrprojekte sind breit gefächert: Diese reichen von der Schaffung österreichweiter Strukturen für die Umsetzung der PädagogInnenbildung Neu, insbesondere gemeinsame Aufnahme- und Auswahlverfahren und gemeinsame IT-Infrastruktur, die über die Zielsetzung einzelner Entwicklungsverbünde hinausgehen, bis hin zur Entwicklung von E-Learning Formaten, Unterstützungsformate für Studierende und Studienwerberinnen und -werber sowie Studien zur Harmonisierung der humanmedizinischen Ausbildung.

\subsubsection{Kooperationen}

Voraussetzung für die Hochschulraumstrukturmittelvergabe 2016 war die Beteiligung von zumindest einer weiteren Institution aus dem Wissenschafts-, Hochschul-, Kunst- oder Kulturbereich oder der Wirtschaft. Wie auch in der HRSM-Ausschreibung 2013 fiel den Projektleiterinnen und -leitern die Suche nach passenden Kooperationspartnerinnen und -partnern (eher) leicht. $63 \%$ bejahten dies in der Online-Befragung, $22 \%$ schätzten die Suche als mittel und lediglich $4 \%$ als schwierig ein. Das mag insbesondere daran liegen, dass die Zusammenarbeit in HRSM-Projekten üblicherweise mit Partnerinnen und Partnern erfolgt, mit denen bereits in der Vergangenheit eine Kooperation bestand.

Darüber hinaus unterscheiden sich die Motive zur Kooperation in den Teilbereichen: Partnerschaften in Projekten zugeordnet zur PädagogInnenbildung Neu waren bereits vor der HRSM-Teilnahme durch die Zuordnung der Hochschulen zu vier regionalen Entwicklungsverbünden (Nord-Ost, Süd-Ost, Mitte, West) relativ klar. Auf der anderen Seite sind Kooperationen im Teilbereich Sonstige Lehrprojekte nicht begrenzt auf Hochschulen im selben Entwicklungsverbund und somit „freier“ in der Partnerinnen- bzw. Partnerwahl. 


\section{Abbildung 73: Suche nach passenden Kooperationspartnerinnen und -partnern}

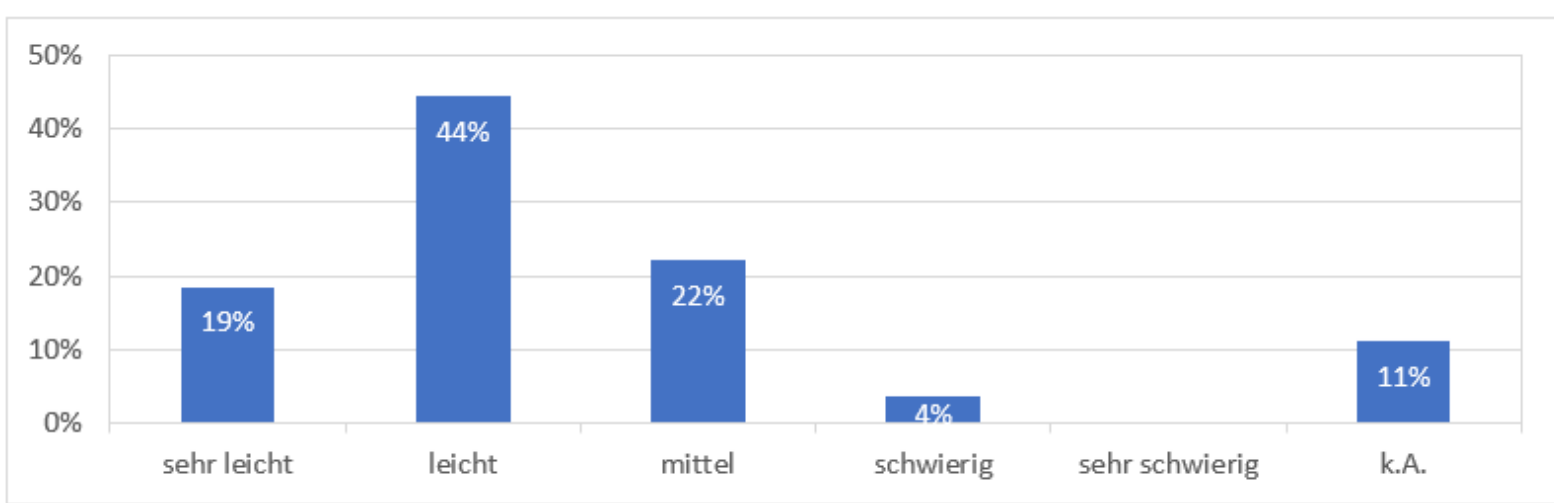

Quelle: WPZ Research Befragung der Projektleiterinnen und -leiter von finanzierten Projekten der HRSM-Ausschreibung 2016, $n=27$

Die insgesamt 32 Projekte der HRSM-Ausschreibung 2016 im Bereich Lehre resultierten in Kooperationen der projektleitenden Universitäten mit 117 Kooperationspartnerinnen und -partnern. 47 \% der finanzierten Projekte (15 Projekte) hatten ein oder zwei Kooperationspartnerinnen und -partner (siehe Abbildung 74). $16 \%$ der Projekte (5 Projekte) hatten lediglich eine Kooperationspartnerin bzw. einen Kooperationspartner. Zwei Kooperationspartnerinnen bzw.-partner sind die häufigste Konstellation (31\% der Projekte) bei Projekten der HRSM-Ausschreibung 2016, gefolgt von Konsortien mit einer projektleitenden Universität und drei Kooperationspartnerinnen und -partnern (19\% der Projekte). Jeweils 12,5\% der Projekte hatten fünf bzw. sechs oder mehr Kooperationspartnerinnen und -partner. Das größte Projekt in dieser Hinsicht war das Projekt „Österreichweites gemeinsames Aufnahme- und Auswahlverfahren für Lehramtsstudien“ unter der Leitung der Universität Graz, welches aus insgesamt 20 Kooperationspartnerinnen und -partnern bestand, gefolgt vom Projekt „Vernetzung leben - Servicezentrum PädagogInnenbildung“ der Universität Salzburg mit acht Kooperationspartnerinnen und -partnern.

\section{Abbildung 74: Anzahl der Kooperationspartnerinnen und -partner der finanzierten Projekte (in absoluten} Zahlen)

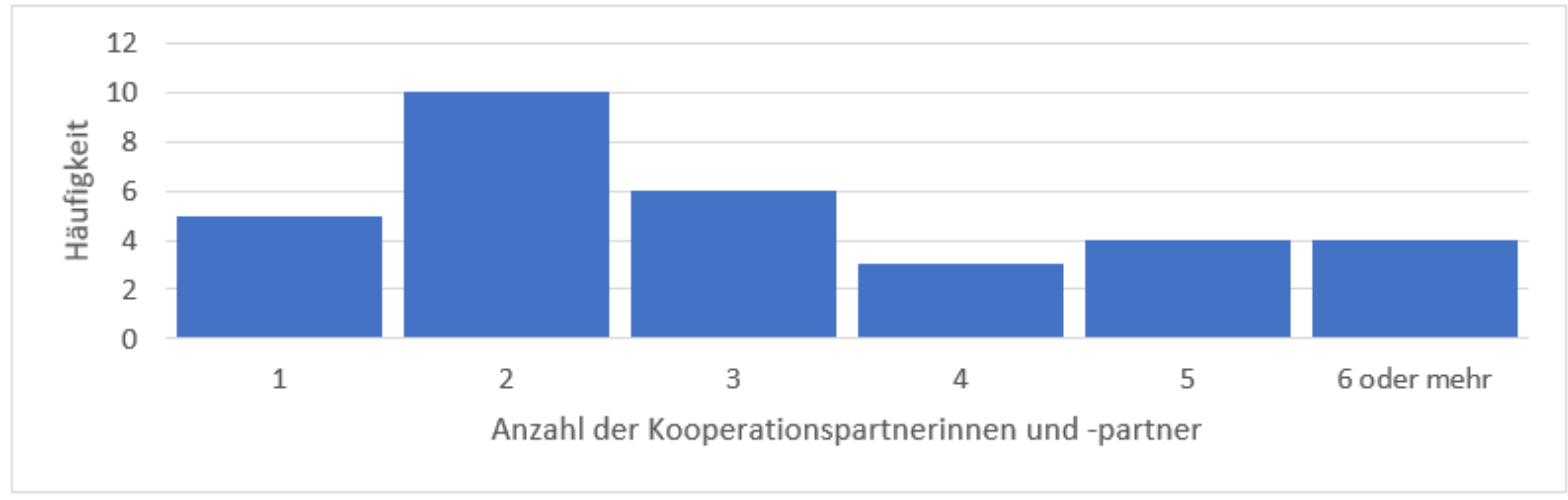

Quelle: BMBWF, eigene Berechnungen; $n=32$

$64 \%$ der finanzierten Projekte (neun Projekte) im Bereich Sonstige Lehrprojekte hatten ein oder zwei Kooperationspartnerinnen und -partner (siehe Abbildung 75), während nur fünf Projekte drei oder mehr Kooperationspartnerinnen und -partner hatten. Im Vergleich dazu hatten Projekte im Bereich PädagogInnenbildung Neu häufiger mehr Kooperationspartnerinnen und -partner. Lediglich 33 \% der Projekte im Bereich PädagogInnenbildung Neu hatten ein oder zwei Kooperationspartnerinnen und -partner. Die am häufigsten vorkommende Konstellation sind drei Kooperationspartnerinnen und -partner (fünf Projekte bzw. $28 \%$ der Projekte in diesem Bereich). Weitere $39 \%$ der Projekte hatten vier, fünf, sechs oder mehr Kooperationspartnerinnen und -partner. Das in dieser Hinsicht größte Projekt ist dem Bereich Sonstige Lehrprojekte 
zuzuordnen, während das größte Projekt im Bereich PädagogInnenbildung acht Kooperationspartnerinnen und Kooperationspartner ausweist.

\section{Abbildung 75: Anzahl der Kooperationspartnerinnen und -partner nach Bereich (in Prozent)}

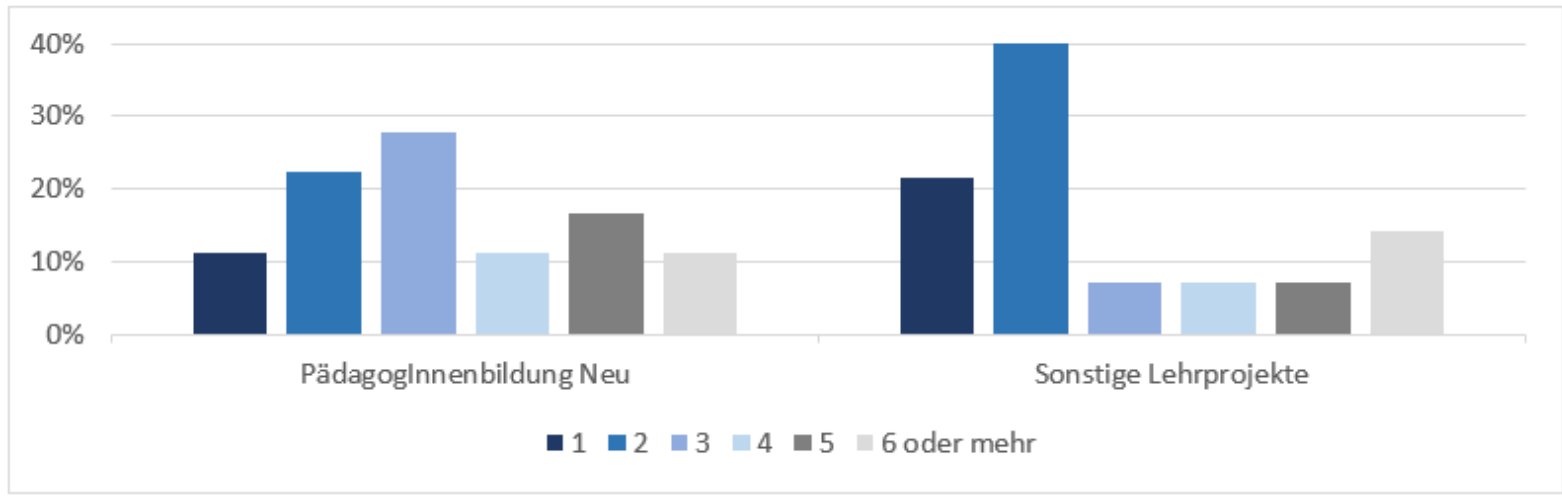

Quelle: BMBWF, eigene Berechnungen; $\mathrm{n}=32$

Verglichen mit der HRSM-Ausschreibung 2013 weisen die Projekte der HRSM-Ausschreibung 2016 einen sehr viel stärkeren Fokus auf Kooperationen mit anderen Universitäten bzw. vor allem mit Pädagogischen Hochschulen auf. Von den insgesamt 117 Kooperationspartnerinnen und -partnern sind $92 \%$ dem Hochschulbereich zuzuordnen (siehe Abbildung 76).

\section{Abbildung 76: Typ und Anteil der Kooperationspartnerinnen und -partner in finanzierten Projekten}

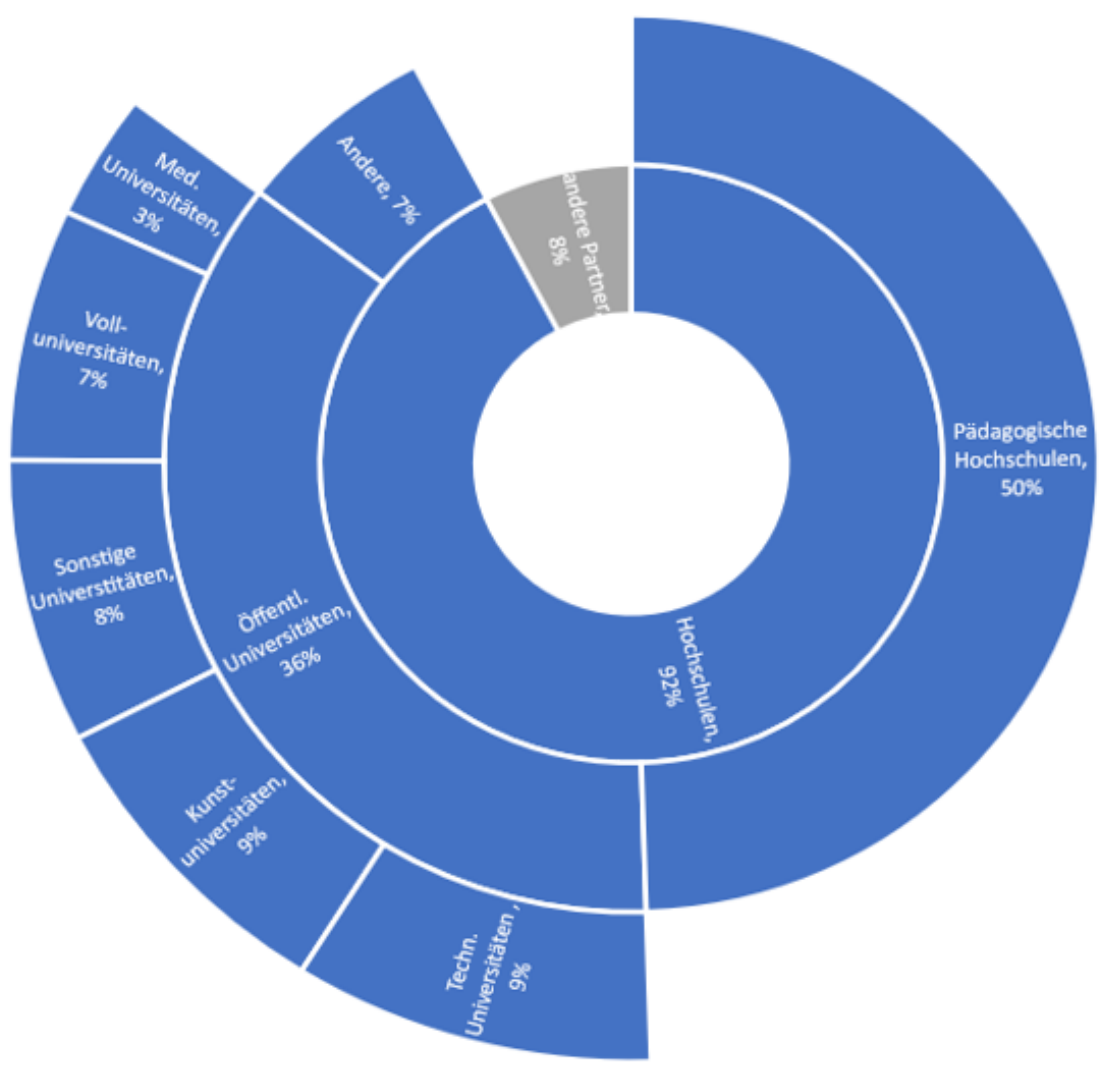

Quelle: BMBWF, eigene Berechnungen; $n=117$ 
Lediglich $8 \%$ der Kooperationspartnerinnen und -partner stammen aus dem nicht-tertiären Sektor, davon $6 \%$ (sieben Kooperationspartnerinnen und -partner) aus dem öffentlichen Sektor (z.B. Bundesländern bzw. nachgestellten Organisationen). Weitere $2 \%$ der Kooperationspartnerinnen und Kooperationspartner stellen Organisationen aus Kunst und Kultur dar, während andere Organisationstypen wie Unternehmen oder Forschungsorganisationen in der HRSM-Ausschreibung $2016 \mathrm{im}$ Bereich Lehre nicht beteiligt waren. Dies kann dadurch erklärt werden, dass Kooperationen mit Unternehmen und anderen Organisationen aus dem nichttertiären Sektor am häufigsten Forschungskooperationen sind, während Wissenschaft-Wirtschaftskooperationen im Bereich der Lehre noch im jungen Entwicklungsstadium sind. ${ }^{23}$

Auch die Zielsetzung der HRSM-Ausschreibung 2016 mit dem Schwerpunkt auf die Umsetzung der PädagogInnenbildung Neu erklärt die Konzentration der Kooperationen auf Hochschulen. Der anteilsmäßig am stärksten vertretene Organisationstyp sind Pädagogische Hochschulen, die insgesamt 50 \% aller Kooperationspartnerinnen und -partner ausmachen. Diese Tatsache entspricht auch den Zielen der HRSM-Ausschreibung 2016, die insbesondere Kooperationen zwischen Universitäten und Pädagogischen Hochschulen in der Umsetzung der PädagogInnenbildung Neu vorsieht. Weitere häufig vertretene Kooperationspartnerinnen und -partner kommen aus dem Sektor der öffentlichen Universitäten (insgesamt $36 \%$ der Kooperationspartnerinnen und Kooperationspartner), verteilt auf Technische Universitäten (9\%), Kunstuniversitäten (9\%), Sonstige Universitäten (8\%) und Volluniversitäten (7\%). Medizinische Universitäten sind in dieser Kategorie am seltensten (mit lediglich $3 \%$ ) vertreten. Auch Privatuniversitäten (5\%) sind recht seltene Kooperationspartnerinnen, wie auch Fachhochschulen ( $2 \%$ bzw. zweimal als Kooperationspartnerinnen) und ausländische Hochschulen (1 \% bzw. einmal als Kooperationspartnerin bzw. -partner), die in der HRSM-Ausschreibung 2016 für Kooperationen im Bereich Lehre offenbar kaum eine Rolle spielten.

\subsubsection{Netzwerkanalyse}

Wie schon für das Jahr 2013 erläutert, lassen sich die Beziehungen der Projektpartnerinnen und -partner durch eine grafische Netzwerkanalyse gut veranschaulichen. Infolge wird daher auch die Anzahl der Verbindungen der verschiedenen Organisationen grafisch beleuchtet.

Tatsächlich zeigen die 32 finanzierten Projekte der HRSM-Ausschreibung 2016 - wie in Abbildung 73 visualisiert - bemerkenswerte Cluster auf. Der Grad der Vernetztheit bzw. des Einflusses einer Organisation („Node“) wird dabei durch die sogenannte Eigenvector Centrality (EC) gemessen. Dieser EC-Wert wird mit einem Wert zwischen 0 und 1 angegeben, wobei 1 auf einen besonders hohen „Einfluss“ im Netzwerk hinweist. Anhand der Breite sowie der Farbe der Verbindungen („Edges“) zwischen den Organisationen lässt sich die Anzahl an Partnerschaften zwischen den zwei „Nodes“ ablesen (siehe Legende). Aus Gründen des stärkeren Fokus auf Hochschulen wurde für die HRSM-Ausschreibung 2016 zudem eine detailliertere Einteilung von Hochschultypen gewählt (siehe Legende unter der jeweiligen Abbildung).

Die im Netzwerk einflussreichsten Organisationen, also jene mit höchsten EC-Werten, weisen die Johannes Kepler Universität Linz mit 1 und das Mozarteum Salzburg mit 0,98 auf. Ebenso hohe Werte weisen die Universität Klagenfurt, die TU Graz, die Pädagogische Hochschule (PH) Tirol sowie die KPH Edith Stein (0.97) auf. Gemessen an der Anzahl an Verbindungen bestehen offenbar besonders enge Partnerschaften zwischen der $\mathrm{PH}$ Oberösterreich und der Privaten PH der Diözese Linz (mit sechs gemeinsamen Projekten) sowie u.a. zwischen der PH Oberösterreich und der PH Salzburg, der PH Niederösterreich und der PH Wien, sowie der PH Oberösterreich und der Universität Salzburg (jeweils fünf).

Dies weist darauf hin, dass Kooperationen zwischen Hochschulen besonders stark von geographischer Nähe getrieben werden, insbesondere durch die Entwicklungsverbünde. Besonders viele Kooperationen entstehen zwischen Hochschulen in derselben Stadt/demselben Bundesland bzw. auch mit Hochschulen in benachbarten Bundesländern. Die Bedeutung der geographischen Nähe für Kooperationen wird auch durch die Anforderungen der PädagogInnenbildung Neu - Umsetzung im regionalen Entwicklungsverbund - verstärkt (siehe hierzu auch die Beschreibungen zu Abbildung 78). Dies ist ersichtlich aus der Breite der Verbindungen („Edges“) zwischen beispielsweise der PH Wien und der PH Niederösterreich, der Universität Graz und der Technischen Universität Graz oder der PH Oberösterreich und der Privaten PH Linz.

\footnotetext{
23 Vgl. Europäische Kommission (2014).
} 
Grafisch lässt sich ein zentrales Cluster zwischen den Pädagogischen Hochschulen (siehe rechte Bildhälfte), den Kunstuniversitäten (siehe untere Bildhälfte und Bildmitte) und den Volluniversitäten erkennen (siehe oberes rechte Bildviertel). Ersteres ist vor allem durch starke Verbindungen von Hochschulen in Salzburg und Linz geprägt. Ebenso lässt sich eine starke Verbindung steirischer und Kärntner Institutionen erkennen (siehe rechte Bildhälfte). Beides spiegelt die Bedeutung der geographischen Nähe bzw. die Umsetzung der Projekte in regionalen Entwicklungsverbünden wider. Eine zentrale verbindende Rolle spielen dabei offenbar die Universität Graz, die Technische Universität Graz, die Universität Linz und die Universität Klagenfurt in der Bildmitte, welche sich nicht eindeutig einem thematischen Cluster zuordnen lassen. Eine stärkere Verbindung durch mehrere Projekte besteht auch zwischen den Pädagogischen Hochschulen Wien und Niederösterreich.

Insbesondere in der oberen Bildhälfte lassen sich Projektverbindungen zwischen Tiroler Universitäten, Hochschulen und Landesakteuren erkennen. In der linken Bildmitte ist die Peripherie durch Wiener Akteure geprägt, während in der unteren Bildhälfte ein Cluster aus den Wiener Kunstuniversitäten mit der University of Westminster und der Vereinigung bildender Künstlerinnen und Künstler Österreichs ersichtlich ist. Diese peripheren Cluster lassen sich, auch aufgrund der relativ kleinen Grundgesamtheit der Projekte, jedoch jeweils eindeutig auf ein bestimmtes Projekt zurückführen. Die recht eindeutige regionale Zuordnung der peripheren Cluster bestätigt auch die oben genannte Bedeutung der geographischen Nähe für Kooperationen.

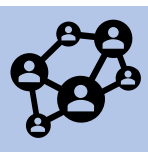

\section{Ausgewählte Projektbeispiele}

Kooperationen im Rahmen der HRSM-Ausschreibung 2016 im Bereich Lehre dienten schwerpunktmäßig der Umsetzung der PädagogInnenbildung Neu und damit der Stärkung der Entwicklungsverbünde zwischen Universitäten und Pädagogischen Hochschulen. Dieser Teilbereich ist geprägt von der Nutzung des HRSMInstruments für die Umsetzung eines strategischen Ziels der Hochschulpolitik. Im Teilbereich Sonstige Lehrprojekte finden sich insbesondere Vorhaben, welche die Digitalisierung für innovative Lehr-/Lernformate nutzten, darüber hinaus aber auch Vorhaben, welche der Harmonisierung von Curricula dienten und auch Entwicklungsverbund-übergreifende Aktivitäten zur Unterstützung der Umsetzung der PädagogInnenbildung Neu.

Kooperationen im Bereich Lehre sollten überdies zur Entwicklung und Umsetzung strategisch wichtiger Vorhaben mit strukturbildender und systemischer Wirkung beitragen und waren folglich Inhalt der Leistungsvereinbarungen. Insbesondere bei den Projekten der PädagogInnenbildung Neu gab es von Beginn an die Intention, die Fortführung durch die Leistungsvereinbarungen abzusichern, Im Hinblick auf Nachhaltigkeit können daher mitunter folgende Projekte der HRSM-Ausschreibung 2016 genannt werden:

Nutzung der HRSM-Finanzierung für die Umsetzung der Reform der LehrerInnenbildung „PädagogInnenbildung Neu“: Während Kooperationen in der HRSM-Ausschreibung 2013 im Bereich Lehre geprägt von thematischer Offenheit waren, wurde das Instrument HRSM 2016 im Bereich Lehre schwerpunktmäßig auf die Umsetzung der PädagogInnenbildung Neu ausgerichtet. Damit wurden die Entwicklungsverbünde gestärkt und Personal- und Infrastrukturaufbau für die im Verbund eingerichteten Lehramtsstudien betrieben. Die Entwicklungsverbünde und Verbundstudien sollen auch in Zukunft bestehen und weiterentwickelt werden, entsprechend sind diese auch Bestandteil der Leistungsvereinbarungen 2019-2021.

Österreichweites gemeinsames Aufnahme- und Auswahlverfahren für Lehramtsstudien: Diese Kooperation wurde durch die HRSM-Ausschreibung 2013 aufgebaut und fand ihre Fortführung in der HRSM-Ausschreibung 2016. Die Partnerschaft umfasst Institutionen aller Entwicklungsverbünde (insgesamt 21 Universitäten und Hochschulen) und soll zu einer besseren Koordination wie auch einheitlichen Abläufen in der Umsetzung der PädagogInnenbildung Neu beitragen.

Verbundinformationssystem - Lehramtsstudien: Unter Beteiligung von Universitäten aller vier Entwicklungsverbünde trägt diese Kooperation zur Entwicklungsverbund-übergreifenden Optimierung von Campusinformationssystemen bei und soll dieses Verbundprojekt daher auch einen wesentlichen Beitrag zur Unterstützung der PädagogInnenbildung Neu leisten. 
Beitrag zur Harmonisierung von Curricula: Die Projekte „Machbarkeitsstudie zur Harmonisierung der humanmedizinischen Ausbildung (Studienjahre 1 - 5) an den öffentlichen Medizinischen Universitäten Österreichs zur Erhöhung der nationalen Mobilität“ und „Microlearning Medizinische Propädeutik: Harmonisierung, Vereinbarkeit und Community Outreach" sind Beispiele für HRSM-finanzierte Kooperationen mit dem Ziel, einen Beitrag zur besseren Abstimmung der Studieninhalte an verschiedenen Standorten zu leisten. So wurde beispielsweise ein gemeinsamer Lernzielkatalog im Rahmen einer Machbarkeitsstudie entwickelt, dessen Weiterentwicklung und Implementierung in der Leistungsvereinbarungsperiode 2019-2021 stattfinden. Ebenso fand z.B. mit der Entwicklung von Microlearning Inhalten (bspw. mini-MOOCs) ein erster Schritt zur curricularen Harmonisierung zwischen den Medizinischen Universitäten statt. 


\section{Abbildung 77: Netzwerkanalyse für Projekte in der HRSM-Ausschreibung 2016}

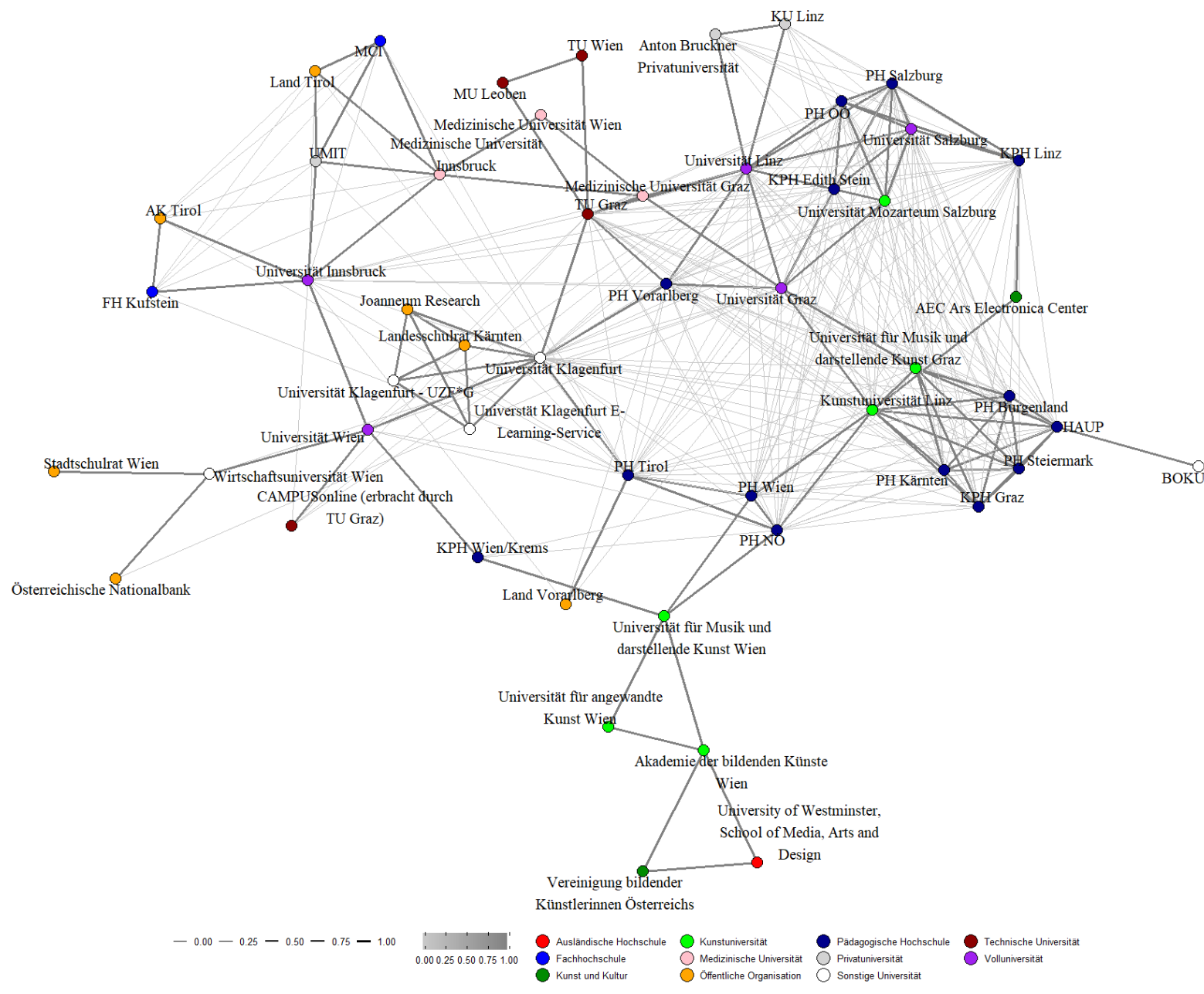

Quelle: Eigene Berechnung und Darstellung; Anmerkung: Projektanzahl=32; Legende: Breite der Verbindungen und Tiefe der Farbe=Anzahl der Projektpartnerschaften, Größe des Rings= Eigenvector Centrality der Nodes 
Kooperationen im Teilbereich PädagogInnenbildung Neu spiegeln den Zusammenschluss der Hochschulen in Entwicklungsverbünden wider, während Kooperationen im Teilbereich Sonstige Lehrprojekte nicht auf die Entwicklungsverbünde beschränkt sind. Um die spezifischen Kooperationsmuster beider Teilbereiche zu analysieren, werden die Ergebnisse in Folge getrennt dargestellt.

\section{Netzwerkanalyse: Teilbereich PädagogInnenbildung Neu}

Die Projekte, die dem inhaltlichen Schwerpunkt PädagogInnenbildung Neu zuzurechnen sind, sind durch die zentralen Rollen der Universitäten in der Vernetzung der Pädagogischen Hochschulen geprägt (siehe Abbildung 78).

Die Netzwerkanalyse veranschaulicht sehr deutlich die Kooperationen in den vier Entwicklungsverbünden (EV) anhand von vier klar abgrenzbaren Clustern. Diese Cluster bzw. Entwicklungsverbünde sind:

- $\quad$ Nord-Ost: Niederösterreich, Wien

- Süd-Ost: Burgenland, Kärnten, Steiermark

- Mitte: Salzburg, Oberösterreich

- West: Tirol, Vorarlberg

Die Netzwerkanalyse zeigt, dass kaum Verbindungen zwischen Verbünden bestehen. Lediglich die Universität Mozarteum Salzburg und die Kirchlich Pädagogische Hochschule Edith Stein (KPH Edith Stein) zeigen angesichts ihrer Beteiligung an beiden Verbünden Verbindungen zu den Entwicklungsverbünden West und Mitte.

\section{Abbildung 78: Netzwerkanalyse für Projekte im Teilbereich PädagogInnenbildung Neu}

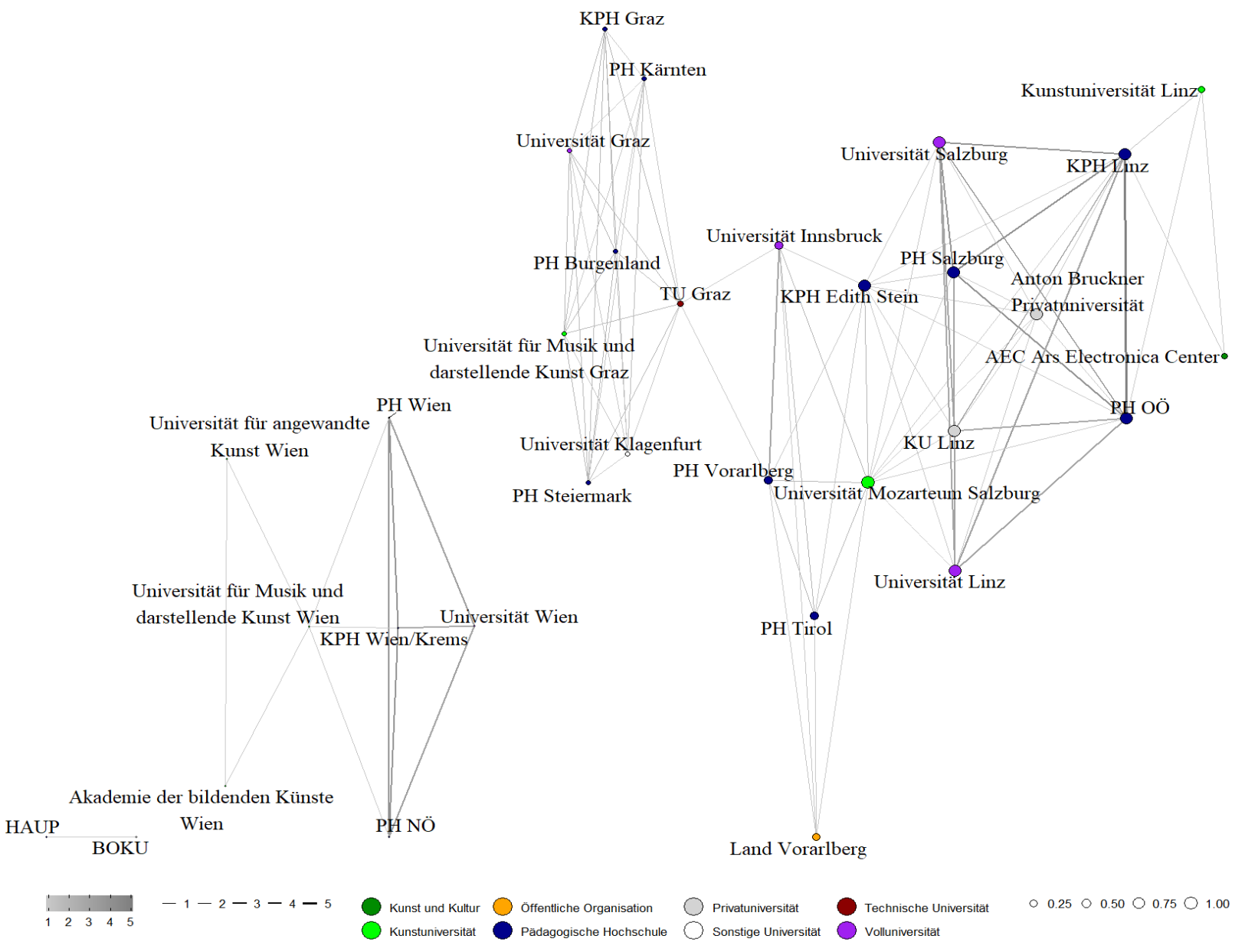

Quelle: Eigene Berechnung und Darstellung; Anmerkung: Projektanzahl=18; Legende: Breite der Verbindungen und Tiefe der Farbe=Anzahl der Projektpartnerschaften, Größe des Rings= Eigenvector Centrality der Nodes 
Im Kontext des HRSM-Instruments unterscheidet sich der Teilbereich PädagogInnenbildung Neu vor allem durch die Vorgabe der Schwerpunktsetzung und der Kooperationsformen von den anderen Bereichen der Ausschreibung. Während im Teilbereich Sonstige Lehrprojekte Kooperationen bottom-up entstehen, sind Universitäten im Teilbereich PädagogInnenbildung Neu angehalten, bestimmte Partnerschaften einzugehen. Auch die inhaltliche Schwerpunktsetzung war durch die Ausschreibung bzw. durch das strategische Ziel der PädagogInnenbildung Neu recht klar definiert: Projekte sollen der Umsetzung im Verbund dienen, beispielsweise durch Personalaufbau, Koordination und administrativ-organisatorische Umsetzung.

Lehramtsstudien (Sekundarstufe) werden seit 2016 im Verbund angeboten. Die Kooperationen im Rahmen der HRSM-Ausschreibung 2016 sind nicht mit Projektende abgeschlossen. Die Partnerschaften bestehen weiter, diese finden auch Verankerung in den Leistungsvereinbarungen 2019-2021. Ebenso ist die Weiterentwicklung der Verbundstudien Teil der Leistungsvereinbarungsperiode. Insgesamt diente das Instrument HRSM im Bereich PädagogInnenbildung der Finanzierung der ersten Phase dieses strategischen Ziels. Der nachhaltige Aufbau der Kooperation zwischen Universitäten und Pädagogischen Hochschulen wurde entsprechend auch strategisch verankert.

\section{Netzwerkanalyse: Teilbereich Sonstige Lehrprojekte}

Für sonstige Lehrprojekte sind regionale Cluster nicht in dem wie bei der PädagogInnenbildung Neu beschriebenen Ausmaß zu erkennen (siehe Abbildung 79). Das Netzwerk ist charakterisiert durch ein zentrales Cluster (rechte Bildhälfte), das sowohl Universitäten verschiedenen Typs als auch Pädagogische Hochschulen umfasst. Ähnlich den Kooperationen der HRSM-Ausschreibung 2013 im Bereich Lehre spielen Organisationen außerhalb des Hochschulbereichs, wie öffentliche Organisationen oder Institutionen im Kunst- und Kulturbereich, nur eine geringe Rolle. Wiederum ähnlich den Kooperationen der HRSM-Ausschreibung 2013 kommt den Universitäten eine zentrale Rolle zu, insbesondere der Technischen Universität Graz zusammen mit der Universität Graz und der Johannes Kepler Universität Linz. Darüber hinaus ist das Gesamtmuster mit der Ausschreibung 2013 vergleichbar: Es gibt ein zentrales Cluster bestehend aus Universitäten und Pädagogischen Hochschulen sowie darüber hinaus einige periphere, thematisch abgrenzbare Verbindungen.

Kooperationen sind - auch hier - häufig geographisch konzentriert, wie z.B. am Standort Graz mit der Universität Graz, der Technischen Universität Graz und der Medizinischen Universität Graz, oder am Standort Tirol, oder entstehen rund um eine thematische Orientierung, wie bei den Medizinischen und Technischen Universitäten. Dieses Muster ähnelt dem der HRSM-Ausschreibung $2013 \mathrm{im}$ Bereich Lehre und deutet auch auf eine Fortführung von Partnerschaften über das Projektende hinweg. Bündnisse wie die TU Austria oder NAWI Graz sind für die Entstehung von weiteren Kooperationen essentiell, so sind auch Projekte wie "MINT-MOOCs der TU Austria“ oder „Optimierung universitätsübergreifender Studienangebote durch E-Learning Maßnahmen bei MINTFächern" aus solchen bereits bestehenden Bündnissen hervorgegangen.

Ähnlich wie bei den Kooperationen der HRSM-Ausschreibung 2013 verfolgen die Projekte im Teilbereich Sonstige Lehrprojekte Aktivitäten, die einen Beitrag zu den strategischen Zielen der Universität leisten sollen. So leistet beispielsweise die Kooperation der Medizinischen Universitäten im Rahmen des Projekts „Machbarkeitsstudie zur Harmonisierung der humanmedizinischen Ausbildung“ einen Beitrag zum Ziel „Implementierung des jeweiligen klinischen Lernzielkatalogs im Prüfungswesen der Medizinischen Universität Graz, Medizinischen Universität Innsbruck, Medizinischen Universität Wien und der Medizinischen Fakultät der JKU“24. Die Weiterentwicklung und weitere Implementierung sind in Folge auch in den Leistungsvereinbarungen 2019-2021 festgehalten ${ }^{25}$.

Trotz der Zuordnung der Projekte im Teilbereich Sonstige Lehrprojekte dienen einige Kooperationen der Unterstützung der Umsetzung der PädagogInnenbildung Neu. Ähnlich wie in der Ausschreibung 2013 sind dies österreichweite Kooperationen in relativ großen Konsortien, die in der Zielsetzung über die einzelnen Entwicklungsverbünde hinausgehen. Diese spezielle Konstellation erklärt auch die recht zentrale Position der Pädagogischen Hochschulen im Netzwerk und das dichte Cluster (rechte Bildhälfte). Konkret handelt es sich um das Projekt "Österreichweites gemeinsames Aufnahme- und Auswahlverfahren für Lehramtsstudien“ unter der Leitung der Universität Graz sowie das Projekt „Verbundinformationssystem - Lehramtsstudien“ unter der

24 Vgl. Leistungsvereinbarung der Medizinischen Universität Graz 2016-2018, S. 58.

25 Siehe z.B. Leistungsvereinbarung der Medizinischen Universität Graz 2019-2021, S. 46. 
Leitung der Universität Klagenfurt. Ersteres ist ein Folgeprojekt der HRSM-Ausschreibung 2013 und stellt eine Fortführung der Partnerschaft über die Jahre hinweg dar. Dies ist insbesondere bemerkenswert, da es sich um das größte Projekt gemessen an der Anzahl an Projektpartnerinnen und -partnern (insgesamt 20) handelt. Die Weiterführung des österreichweiten Aufnahme- und Auswahlverfahrens ist nach Projektende 2018 Bestandteil der Leistungsvereinbarung der Universität $\mathrm{Graz}^{26}$. Beide Projekte unterstützen durch systemische Maßnahmen die Umsetzung der neuen Verbundstudien für Lehramt und sollen insbesondere organisatorische Prozesse über Entwicklungsverbünde hinweg optimieren.

\section{Abbildung 79: Netzwerkanalyse für Projekte im Teilbereich Sonstige Lehrprojekte}

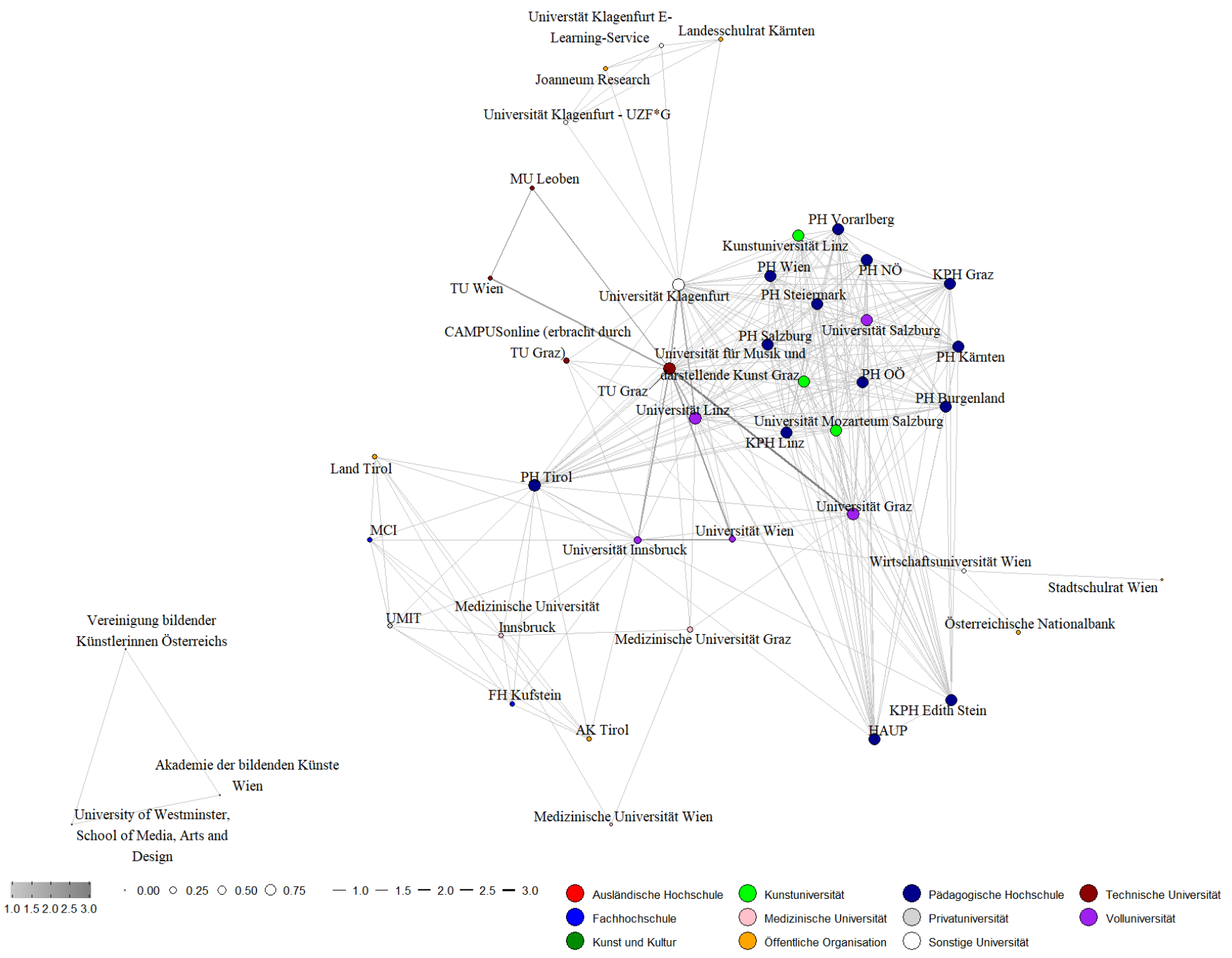

Quelle: Eigene Berechnung und Darstellung: Anmerkung: Projektanzahl=14; Legende: Breite der Verbindungen und Tiefe der Farbe=Anzahl der Projektpartnerschaften, Größe des Rings= Eigenvector Centrality der Nodes

\subsection{Zur Zielerreichung und Wirkung}

Fast alle Projektleiterinnen und -leiter (92\%) sind im Rahmen der Online-Befragung der Meinung, dass ihr jeweiliges HRSM-Projekt die grundsätzliche Intention erfüllt hat, lediglich $8 \%$ waren anderer Meinung. Die Ziele, die mit den HRSM-Projekten verfolgt wurden, waren sehr divers. Das zeigt sich auch am hohen Anteil der

26 Siehe Leistungsvereinbarung der Universität Graz 2019-2021, S. 48. 
Antwortkategorie „nicht zutreffend/ weiß nicht“, die von den Leiterinnen und Leitern immer dann angegeben wurde, wenn ein Ziel für ihr Projekt nicht relevant war. Am häufigsten wurde angegeben, dass der Aufbau neuer hochschulübergreifender Studienangebote (inkl. Lehramtsstudien) sehr gelungen ist, dahinter folgen die Stärkung der Verbundregionen zur Durchführung von Lehramtsstudien und der Einsatz von E-Learning Formaten. Bei der Verbesserung der innerösterreichischen Mobilität von Studierenden gaben $10 \%$ der Leiterinnen und Leiter an, dass dies gar nicht gelungen ist. Das ist die einzige Zieldimension, in der eine negative Antwortkategorie verwendet wurde (siehe Abbildung 80).

Wird die Zielerreichung nach inhaltlichen Schwerpunkten der Projekte differenziert, zeigt sich, dass bei Projekten im Bereich der PädagogInnenbildung Neu insbesondere die Gewährleistung und Verbesserung der Qualität der Lehre in Lehramtsstudien, der Aufbau neuer hochschulübergreifender Studienangebote, die Weiterentwicklung von hochschulübergreifenden Studienangeboten und die Stärkung der Verbundregionen zur Durchführung von Lehramtsstudien sehr gelungen sind. Bei sonstigen Lehrprojekten waren es dagegen besonders Innovationen und fachliche Neuerungen sowie der Auf- und Ausbau von Infrastrukturen zwecks Nutzung der Digitalisierung in der Lehre, die als sehr gelungen wahrgenommen werden.

\section{Abbildung 80: Projektziele}

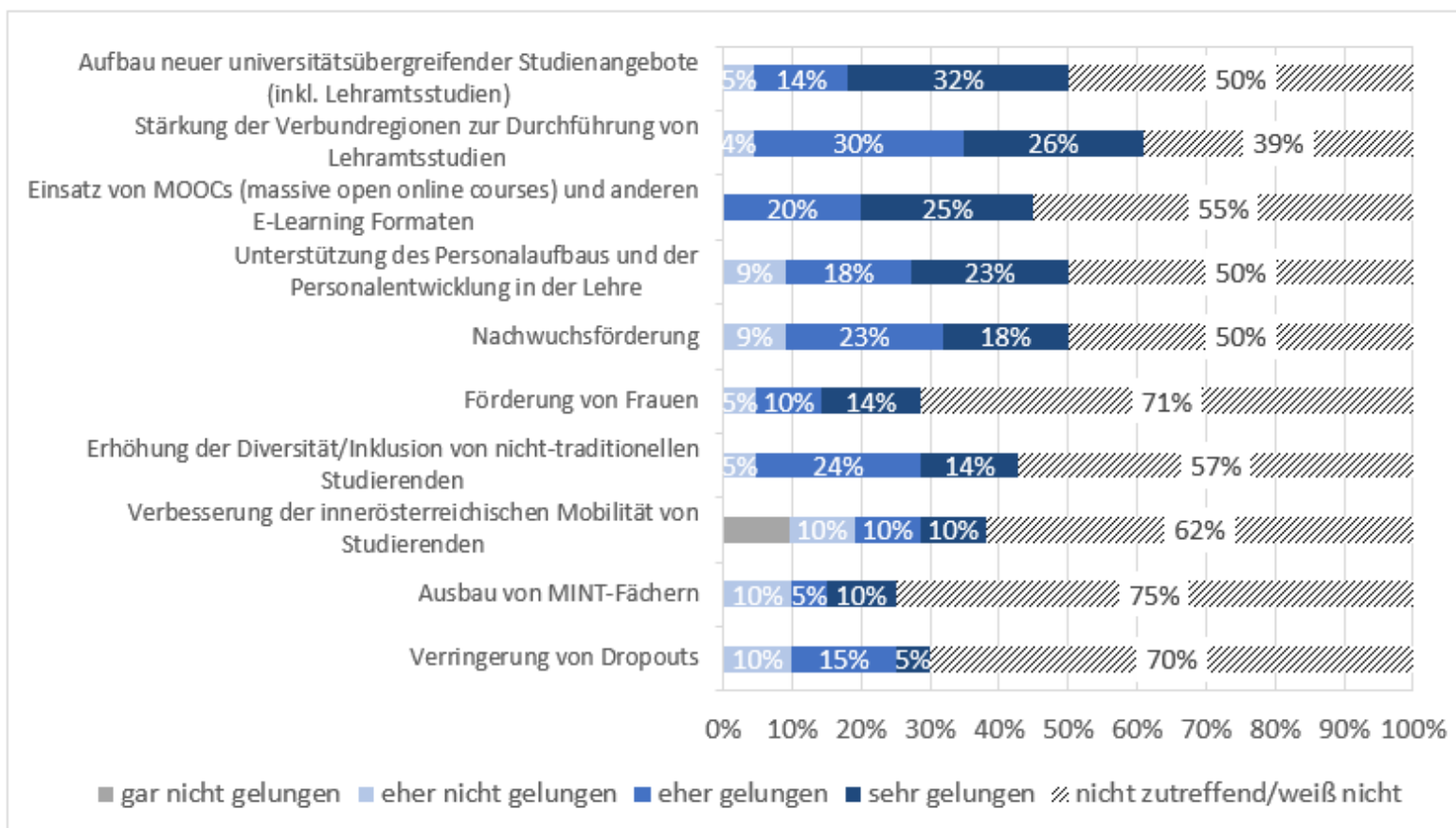

Quelle: WPZ Research Befragung der Projektleiterinnen und -leiter von finanzierten Projekten der HRSM-Ausschreibung 2016, $\mathrm{n}=22$

Abseits dieser Projektziele waren es insbesondere zwei Nutzenaspekte, welche die HRSM-2016-Projekte im Bereich Lehre aus Sicht der Projektleiterinnen und -leiter angestoßen haben. $85 \%$ waren der Meinung, dass die Projekte die nationale oder internationale Sichtbarkeit der Universität verbessert haben, und $81 \%$ waren der Ansicht, dass auch Synergieeffekte erzielt werden konnten. 


\section{Abbildung 81: Zusatznutzen}

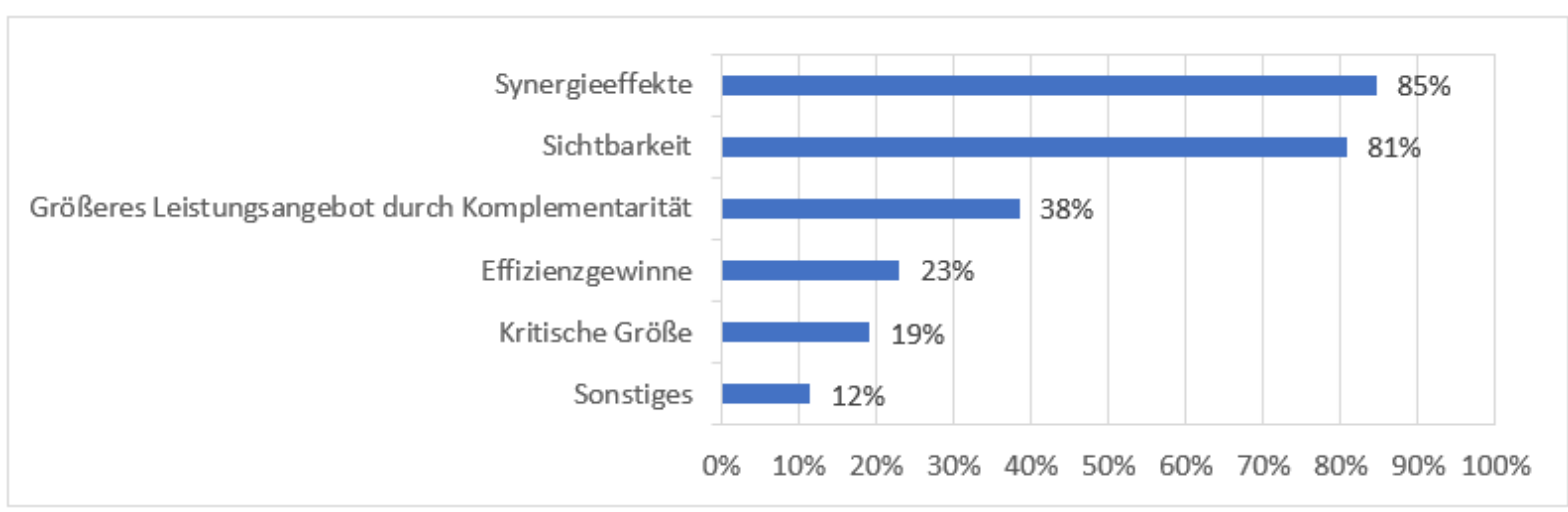

Quelle: WPZ Research Befragung der Projektleiterinnen und -leiter von finanzierten Projekten der HRSM-Ausschreibung 2016, $\mathrm{n}=26$

Neben den dargestellten Zielen und Nutzen waren die Projektleiterinnen und -leiter in der Online-Befragung außerdem der Meinung, dass die HRSM-2016-Projekte einen Beitrag zur Profilbildung der jeweiligen Universität geleistet haben. 54 \% gaben an, dass die Projekte eher einen Beitrag geleistet haben, 31 \% waren der Meinung, dass die Projekte sehr zur Profilbildung beigetragen haben.

\section{Abbildung 82: Beitrag zur Profilbildung der Universität}

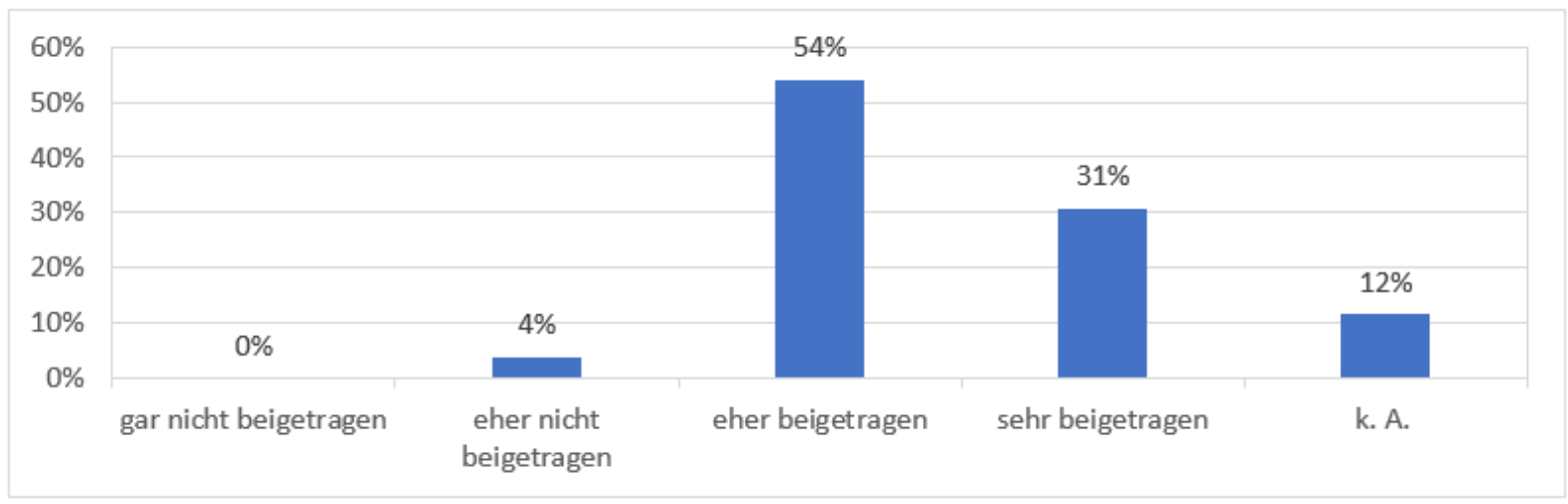

Quelle: WPZ Research Befragung der Projektleiterinnen und -leiter von finanzierten Projekten der HRSM-Ausschreibung 2016, $\mathrm{n}=26$

\section{Abbildung 83: Abgestimmter Hochschul- und Forschungsraum}

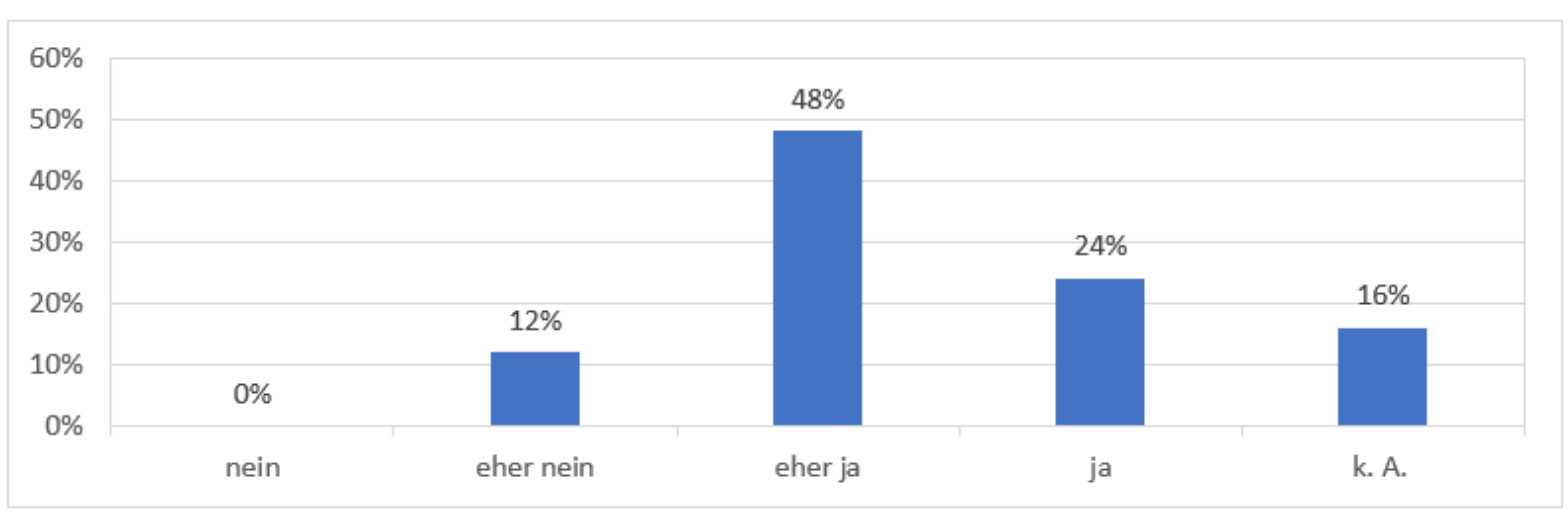

Quelle: WPZ Research Befragung der Projektleiterinnen und -leiter von finanzierten Projekten der HRSM-Ausschreibung 2016, $\mathrm{n}=25$ 
Wie in Abbildung 83 dargestellt, waren die Projektleiterinnen und -leiter auch der Meinung, dass die Projekte der HRSM-Ausschreibung 2016 im Bereich Lehre (tendenziell/eher) zu einem abgestimmten Hochschul- und Forschungsraum beigetragen haben. $72 \%$ teilten diese Einschätzung, lediglich $12 \%$ waren (eher) anderer Meinung.

Mit $60 \%$ war etwas mehr als die Hälfte der Projektleiterinnen und -leiter der Meinung, dass die Nachhaltigkeit der HRSM-2016-Projekte (sehr) hoch war. Weitere $28 \%$ schätzten die Nachhaltigkeit als mittelmäßig und lediglich $4 \%$ als niedrig ein. Leiterinnen und Leiter von Projekten, die im Bereich der PädagogInnenbildung Neu angesiedelt waren, gaben eine deutlich höhere Nachhaltigkeit an. Die Antwortkategorie "sehr hoch" wurde um 30 Prozentpunkte mehr angegeben als bei sonstigen Lehrprojekten (siehe Abbildung 84). Dieses Antwortverhalten ist insbesondere rückführbar auf die Verankerung aller Vorhaben im Bereich der PädagogInnenbildung Neu in den Leistungsvereinbarungen 2019-2021. Im Bereich Sonstige Lehrprojekte wurde die Fortführung von Vorhaben auch in der Leistungsvereinbarung 2019-2021 verankert, jedoch nicht in dem systematischen Ausmaß wie im Bereich PädagogInnenbildung Neu.

Die Nachhaltigkeit der Projektergebnisse wurde - nach eigenen Angaben - insbesondere durch die Verankerung der Projekte in den Leistungsvereinbarungen (68\%) sichergestellt. Dahinter folgten mit jeweils $48 \%$ Veränderungen von Strukturen bzw. Prozessen und weitere Kooperationsprojekte.

\section{Abbildung 84: Nachhaltigkeit}

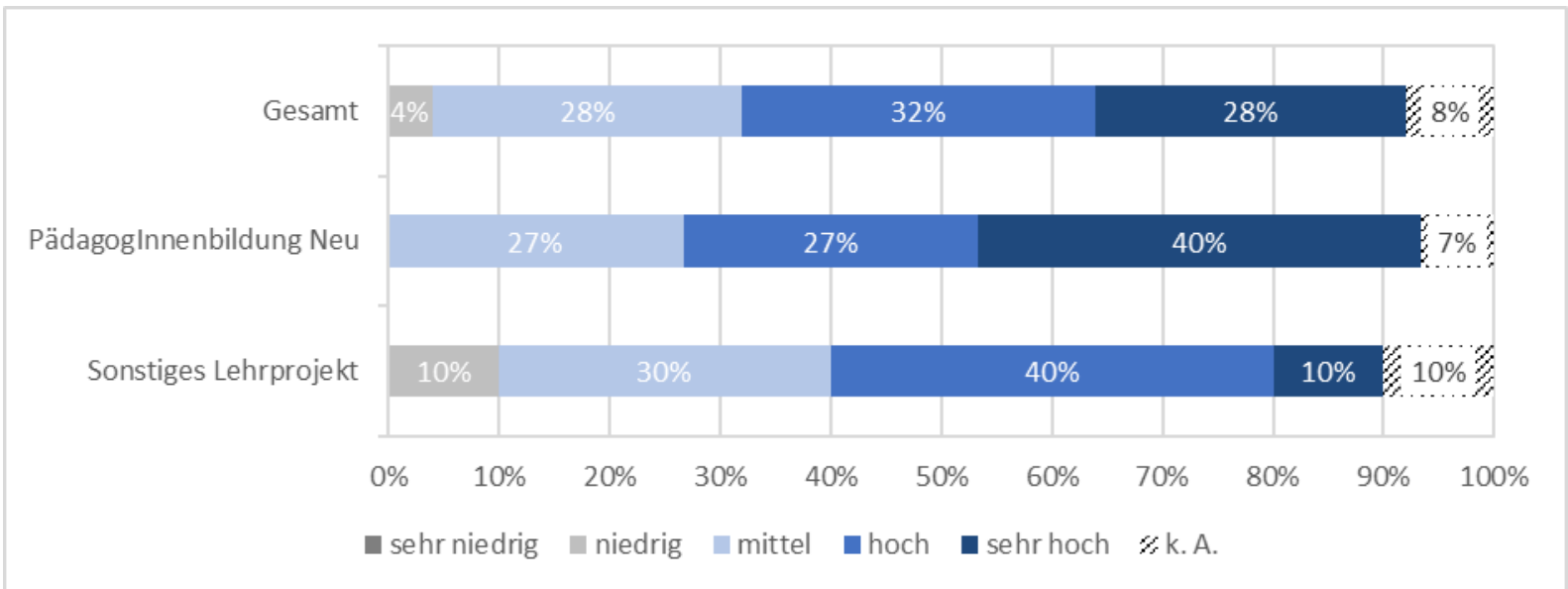

Quelle: WPZ Research Befragung der Projektleiterinnen und -leiter von finanzierten Projekten der HRSM-Ausschreibung 2016, $\mathrm{n}=25$

Wie auch bei der HRSM-Ausschreibung 2013 unterscheidet sich die Einschätzung der Nachhaltigkeit etwas anhand des Universitätstyps. Auch bei den HRSM 2016 gaben die Leiterinnen und Leiter von Projekten, die an Kunstuniversitäten durchgeführt wurden, deutlich seltener an, dass die Nachhaltigkeit (sehr) hoch gewesen sei. Nur eine bzw. einer von drei Projektleiterinnen und Projektleitern waren dieser Meinung. Bei den übrigen Universitätstypen gab es dagegen kein stark abweichendes Antwortverhalten.

Eine weitere Dimension der Nachhaltigkeit zeigte die Frage, ob innovative Ansätze aus den HRSM-2016-Projekten in den Regelbetrieb der Universitäten übernommen wurden. Wie in Abbildung 85 dargestellt, bejahten mit $96 \%$ fast alle Projektleiterinnen und -leiter diese Frage. Folglich wurden innovative Ideen und Ansätze, die im Rahmen der HRSM-Projekte erprobt wurden, an (nahezu) allen Universitäten übernommen bzw. weitergeführt. 


\section{Abbildung 85: Übernahme von innovativen Ansätzen in den Regelbetrieb}

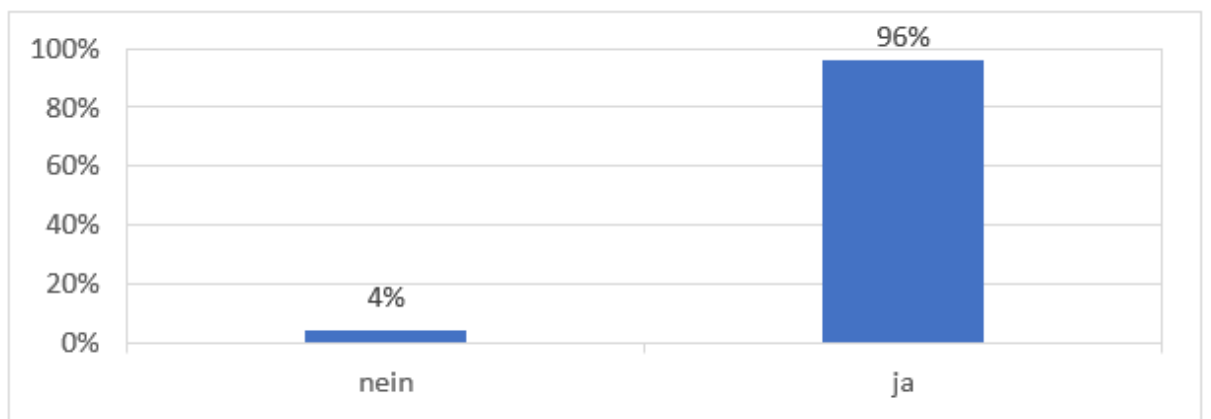

Quelle: WPZ Research Befragung der Projektleiterinnen und -leiter von finanzierten Projekten der HRSM-Ausschreibung 2016, $\mathrm{n}=25$

Hätten die Universitäten die HRSM-Finanzierung des BMBWF nicht erhalten, so hätten sie die Projekte nicht im selben Ausmaß durchführen können. Ein Drittel der befragten Projektleiterinnen und -leiter gab an, dass es das jeweilige Projekte ohne HRSM-Finanzierung gar nicht hätte durchführen können. $60 \%$ glauben, dass sie ihr Kooperationsprojekt zwar durchführen hätten können, allerdings nur in einem geringeren Umfang. Mit $4 \%$ war lediglich ein verschwindend geringer Anteil der Meinung, dass das Kooperationsprojekt auch ohne HRSMFinanzierung zu Stande gekommen wäre. Tatsächlich bestanden große Unterschiede im Antwortverhalten zwischen den inhaltlichen Schwerpunkten der Projekte. Im Bereich der sonstigen Lehrprojekte waren die Leiterinnen und Leiter zu $60 \%$ davon überzeugt, dass ihr Projekt ohne HRSM-Finanzierung gar nicht zu Stande gekommen wäre, im Bereich der PädagogInnenbildung Neu waren es hingegen nur $20 \%$ und damit um 40 Prozentpunkte weniger. Gefiltert nach Universitätstyp sind verstärkt die Sonstigen Universitäten der Meinung, dass sie die Projekte ohne HRSM-Finanzierung nicht verwirklichen hätten können.

\section{Abbildung 86: Kooperationen auch ohne die HRSM-2016-Finanzierung bzw. das HRSM-2016-Projekt}

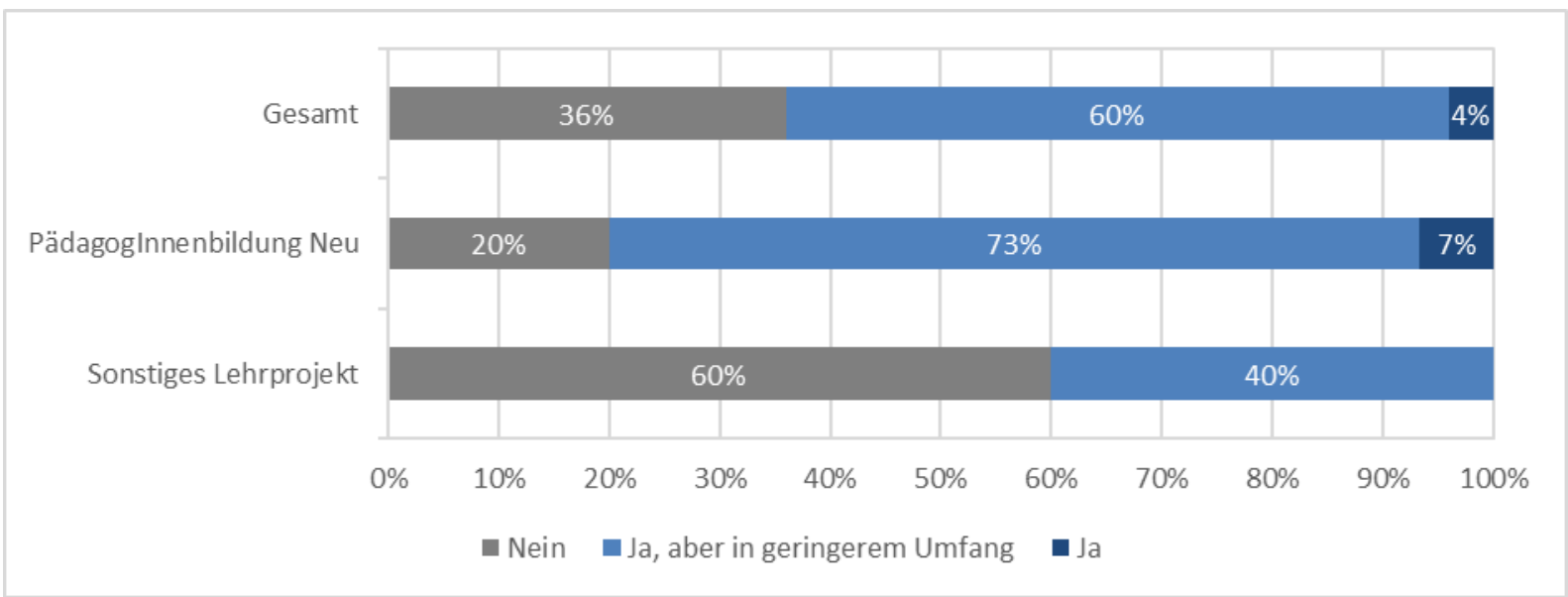

Quelle: WPZ Research Befragung der Projektleiterinnen und -leiter von finanzierten Projekten der HRSM-Ausschreibung 2016, $\mathrm{n}=25$

Aufgrund ihrer Erfahrungen in der Beantragung und Durchführung der HRSM-2016-Projekte im Bereich Lehre würde sich mit $84 \%$ eine überwiegende Mehrheit der Projektleiterinnen und -leiter wieder an einer HRSMAusschreibung bzw. einer vergleichbaren Ausschreibung beteiligen wollen. 
Abbildung 87: Teilnahme an einer zukünftigen HRSM-Ausschreibung bzw. einer vergleichbaren Ausschreibung

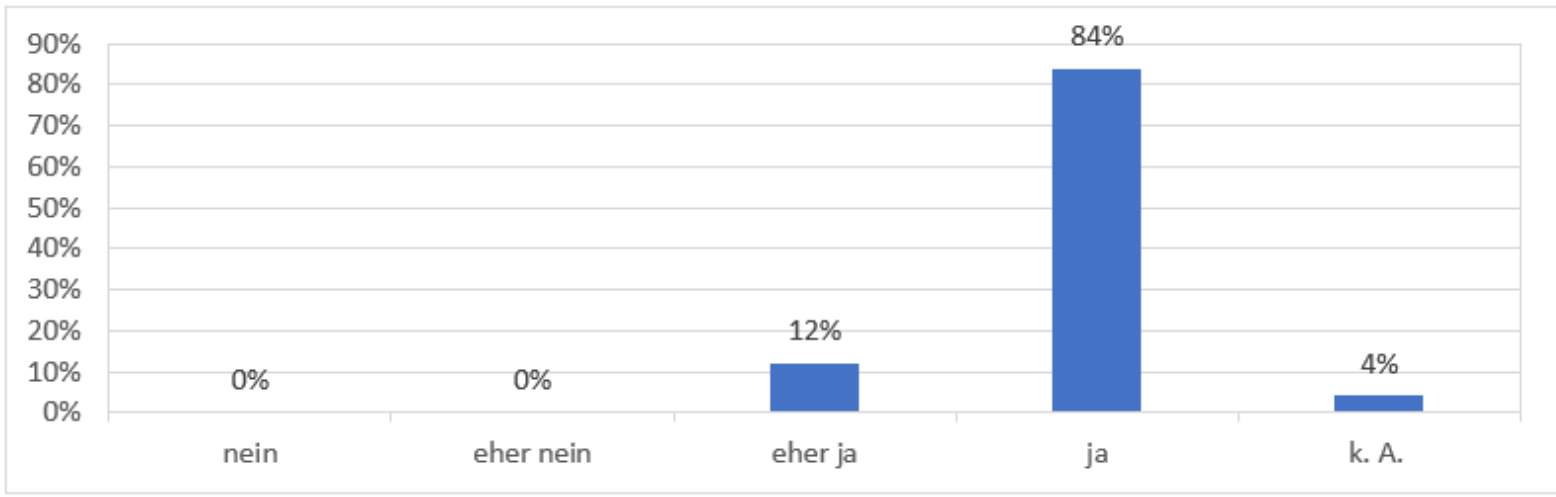

Quelle: WPZ Research Befragung der Projektleiterinnen und -leiter von finanzierten Projekten der HRSM-Ausschreibung 2016, $\mathrm{n}=25$ 


\section{Reflexion, Synthese und Empfehlungen}

Im letzten Kapitel der Evaluierungsstudie werden die mittels Daten- und Dokumentenanalysen und via OnlineBefragung der Projektleiterinnen und -leiter gewonnenen Befunde und Erkenntnisse zur HRSM-Ausschreibung 2013 und zur HRSM-Ausschreibung 2016 im Bereich Lehre zusammengetragen und unter Einbeziehung von vertiefenden Interviews mit Projektleiterinnen und Projektleitern sowie Gesprächen mit Expertinnen und Experten reflektiert. Im zweiten Abschnitt werden sodann die wichtigsten Evaluierungsergebnisse in einer Synthese zusammengefasst und vor diesem Hintergrund Empfehlungen für weitere HRSM-Ausschreibungen abgeleitet.

\subsection{Reflexion und Synthese}

Um für einige zentrale Ergebnisse ein besseres Verständnis zu schaffen bzw. Befunde aus den Befragungen zu vertiefen, wurden in den Monaten September und Oktober 2020 insgesamt 17 Gespräche mit ausgewählten Projektleiterinnen und Projektleitern sowie Expertinnen und Experten geführt. Zu den Interview- bzw. Gesprächspartnerinnen und -partnern zählten einerseits Personen, welche in der Auswahlkommission der HRSM-Ausschreibungen 2013 und 2016 (d.h. Vertretungen seitens BMBWF, BMF und uniko) involviert waren, sowie andererseits Projektleiterinnen und Projektleiter beider Ausschreibungsrunden, wobei hier auch Universitätsleitungen einbezogen wurden. Aufgrund der Covid-19-Pandemie fand die Mehrzahl der Gespräche in einem Online-Format statt. Im Zentrum der Gespräche standen drei Fragestellungen:

- Was machen „echte“ bzw. „gute“ Kooperationen unter den Universitäten aus? Gibt es hierfür Kriterien? Wie zeigen sich solche Kooperationen? Anhand welcher Erfolgskriterien/Dimensionen kann man solche Kooperationen festmachen?

- Wo gibt es noch Potentiale in der Unterstützung von Kooperationen zwischen Österreichs Universitäten bzw. Hochschulen?

- Worin liegt der Hebel in der HRSM-Finanzierung? Was kann man in Zukunft noch besser machen?

Vorherige Zusammenarbeit und eine Vertrauensbasis wurden durchgehend als wichtigste Faktoren einer erfolgreichen Kooperation genannt, weshalb ein Großteil der HRSM-Projekte mit bestehenden Kooperationspartnerinnen und -partnern erfolgt ist. Darüber hinaus motivieren auch gemeinsame Schwerpunktthemen, komplementäre Kompetenzen bzw. Expertisen und das Interesse an einer gemeinsamen Ausrichtung universitätsübergreifende Kooperationen. Dass gerade die geographische Nähe hierbei von großem Vorteil gesehen wird - nach wie vor wird vor allem der persönliche Austausch als wichtig für eine gute Zusammenarbeit erachtet - wird von vielen Projektleiterinnen und -leitern hervorgehoben.

Tatsächlich wird eine vorherige Kooperation als essentieller Ausgangspunkt für eine gute Kooperation im Rahmen von HRSM-Projekten erachtet, damit fand auch die Partnerinnen- bzw. Partnerwahl in der Antragsphase sehr bewusst statt. Seitens der Auswahlkommission wurde durchaus auf das Potential der jeweiligen Kooperationen geachtet. Die Bezugnahme auf bereits bestehende Netzwerke, gemeinsame Forschungsprojekte, die gemeinsame Nutzung von Infrastrukturen, gemeinsame Projektvorhaben, eine gemeinsame strategische Zielrichtung usw. wurden als Indiz für gute Kooperationen gewertet.

War es Ziel der HRSM-Ausschreibung 2013, auch mit Unternehmen zu kooperieren, so muss klar festgehalten werden, dass die HRSM hierfür ein nicht adäquates Format darstellen. Tatsächlich gibt es in Österreich eine Fülle von Förderprogrammen, die auf eine Unterstützung von Unternehmen, die sich in Wissenschaft-Wirtschaftskooperationen engagieren, abzielen. Das Instrument der HRSM kann im Vergleich hierzu den Unternehmen in der Rolle der Kooperationspartnerin bzw. des Kooperationspartners keinerlei Unterstützung anbieten. Angesichts dessen gab es auch kaum Projekte im Rahmen der HRSM-Ausschreibung 2013, die eine Unternehmenskooperation vorweisen. Seitens des BMBWF hat man diese Gegebenheit erkannt und ist man von dem Ziel, durch HRSM auch Kooperationen mit Unternehmen zu forcieren, in den nächsten Ausschreibungen abgewichen.

Im Gegensatz zur HRSM-Ausschreibung 2013, die völlig themenoffen, bottom-up gerichtet als Anschubfinanzierung für hochschulübergreifende Kooperationen in den Bereichen Lehre, Forschung und Verwaltung konzipiert war, hatte die HRSM-Ausschreibung 2016 ein klares Ziel, nämlich die Einführung der PädgogInnenausbildung $\mathrm{Neu}$ zu unterstützen. Probleme in der Umsetzung sind dabei eher in den unterschiedlichen 
Charakteristika der Zusammenführung von unterschiedlichen Hochschultypen zu sehen als im Instrument der HRSM-Finanzierung selbst.

Tatsächlich nahm die Mehrzahl der HRSM-Projekte ihren Ausgangspunkt in bereits bestehenden Kontakten, Partnerschaften und Netzwerken. Die HRSM wurden in diesem Kontext auch als Möglichkeit gesehen, bestehende Partnerschaften zu stärken, weiter auszubauen. Die finanziellen Mittel durch die HRSM wurden für den Auf- und weiteren Ausbau als durchwegs essentiell angesehen. So gibt es auch durchaus Kooperationen, wie z.B. im Bereich der Lehre, die von der HRSM-Ausschreibung 2013 ausgehend, über die HRSM-Ausschreibung 2016 bis hin zur HRSM-Ausschreibung 2019 unter dem Aspekt der digitalen Transformation weitergetragen bzw. weiterentwickelt wurden.

\section{Ergänzung: Vergleich mit Kooperationsmustern in den Spezialforschungsbereichen des FWF}

Ein Vergleich mit Kooperationsmustern in den Spezialforschungsbereichen des FWF, dem Programm zur Förderung von Forschungsnetzwerken in der Grundlagenforschung, zeigt, dass zwischen den beiden Instrumenten ähnliche Muster ersichtlich sind. Der Einfluss der geographischen Nähe sowie der thematischen Orientierung auf Kooperationen ähnelt Forschungskooperationen in den Spezialforschungsbereichen. Die gemeinsame Einrichtung eines Spezialforschungsbereichs wird, ähnlich wie bei HRSM-Projekten im Bereich Forschung, determiniert durch denselben geographischen Standort (z.B. Graz, Wien) und eine ähnliche thematische Orientierung (allgemein/medizinisch/technisch). Während ein kausaler Effekt nicht nachweisbar ist, sind die in HRSM-Projekten stattfindenden Kooperationen durchaus auch in den Netzwerken der Spezialforschungsbereiche zu beobachten. Dazu zählen beispielsweise die Zentralität der Universität Wien im Netzwerk und die enge Kooperationstätigkeit unter Grazer bzw. Wiener Universitäten. Zwar sind die beiden Instrumente nicht direkt vergleichbar, da die Spezialforschungsbereiche insbesondere im Bereich der Exzellenz wirken, so lässt sich aus dem Vergleich dennoch schließen, dass HRSM-Projekte im Bereich Forschung existierende Kooperationsverhalten zumindest bestärken. Eine disziplinäre Netzwerkanalyse war durch die gegebenen Projektdaten nicht möglich, jedoch lassen sich durch die Analyse der Projektdokumente auch gewisse disziplinäre Ähnlichkeiten feststellen: Ähnlich wie in den Spezialforschungsbereichen lässt sich die Mehrheit der HRSMProjekte disziplinär den Naturwissenschaften, insbesondere den Life Sciences, zuordnen. Dies spricht ebenso für die Wirkung der HRSM im Bereich der Stärkung von existierenden Partnerschaften und Schwerpunktthemen.

Die strategische Bedeutung der HRSM-Projekte wurde seitens der Projekteiterinnen und -leiter unterstrichen, indem diese (wenn auch für das Evaluierungsteam nicht immer ersichtlich) in den Leistungsvereinbarungen verankert waren bzw. sind. Auf die Frage, warum für die HRSM-Finanzierung abgelehnte Projekte, mehrheitlich finanziert durch das Globalbudget, wenn auch nicht im vollem Umfang dennoch durchgeführt wurden, hielten die befragten Universitätsleitungen fest, dass dieses Faktum als Indiz für einen hohen strategischen Stellenwert der beantragten Projekte zu werten ist.

Durch HRSM-Mittel finanzierte Kooperationen leisten einen wichtigen Beitrag zur Erfüllung der strategischen Ziele der Universitäten. Darüber hinaus trugen die HRSM-Projekte zur Profilbildung der Universitäten bei und leisteten diese einen Beitrag zu einem besser abgestimmten österreichischen Hochschul- und Forschungsraum. Die systemische Wirkung lässt sich auch auf Ebene der einzelnen Vorhaben und Projektziele erkennen. Insgesamt wurden die Projektziele in einem hohen Ausmaß erfüllt. Im Rahmen der HRSM-Ausschreibung 2013 erwirkten die finanzierten Projekte insbesondere eine Erhöhung der Sichtbarkeit (universitätsintern wie auch -extern), die Stärkung von Exzellenz sowie den Aufbau nachhaltiger Kooperationsstrukturen. Projekte im Bereich Lehre trugen insbesondere zum Auf- und Ausbau von Forschungsinfrastruktur wie auch zum Ausbau bestehender Forschungsthemen/Forschungsgruppen bei. Projekte im Bereich Lehre erreichten insbesondere Zielsetzungen zur Erhöhung der Sichtbarkeit und Weiterentwicklung bestehender Lehrangebote. Projekte im Bereich Verwaltung erzielten insbesondere eine Verbesserung von Verwaltungsstrukturen und -prozessen und trugen ebenso zu einer erhöhten Sichtbarkeit bei. 
Anders in der HRSM-Ausschreibung 2016 im Bereich Lehre: Hier trugen die finanzierten Projekte besonders häufig zum Aufbau neuer hochschulübergreifender Studienangebote (inkl. Lehramtsstudien), zur Stärkung von Verbundregionen zwecks Durchführung von Lehramtsstudien und zum vermehrten Einsatz von E-Learning Formaten bei. Projekte im Bereich der PädagogInnenbildung Neu verwiesen auf die Gewährleistung und Verbesserung der Qualität der Lehre in Lehramtsstudien, den Aufbau neuer hochschulübergreifender Studienangebote, die Weiterentwicklung von hochschulübergreifenden Studienangeboten sowie die Stärkung von Verbundregionen zur Durchführung von Lehramtsstudien. Projekte im Bereich Sonstige Lehrprojekten konnten demgegenüber insbesondere Innovationen und fachliche Neuerungen sowie den Auf- und Ausbau von Infrastrukturen zwecks Nutzung der Digitalisierung in der Lehre erzielen.

Die Nachhaltigkeit von HRSM-finanzierten Kooperationen und damit einhergehend die Sichtbarkeit von HRSMProjektergebnissen können vor allem mit den zugrundeliegenden Netzwerken begründet werden. Tatsächlich ist ein Großteil der HRSM-Kooperationen aus bestehenden Kontakten bzw. Partnerschaften in der Vergangenheit hervorgegangen. Als Beispiele können hierfür die TU Austria, NAWI Graz oder BioTechMed Graz genannt werden. Durch die HRSM-Finanzierung wurden diese bestehenden Partnerschaften und Netzwerke weiters gestärkt.

Einige Interviewpartnerinnen und -partner verwiesen in diesem Kontext auch wieder auf die Bedeutung von persönlichen Kontakten und damit einhergehend auf die Inanspruchnahme weiterer HRSM-Ausschreibungen bzw. auch anderer Förderlinien und -formate, um den Fortbestand bzw. die Nachhaltigkeit der Kooperationen abzusichern.

In der Nachhaltigkeit bzw. in der Hebelwirkung durch Komplementaritäten und der Nutzung von Synergien wird das größte Potential von universitätsübergreifenden Kooperationen gesehen. Als bedeutsam wird auch der Grad des Willens bzw. des Engagements gesehen, sämtliche Potentiale zu heben, nicht zuletzt durch HRSM-finanzierte Projekte systemische - oftmals im Sinne von „systemverändernd“ - Wirkungen herbeizuführen. Hierzu bedarf es klarer Zielsetzungen und auch einer klaren Kommunikation dieser - beides wird sowohl von den befragten Projektleiterinnen und Projektleitern wie auch von den befragten Hochschulleitungen als begrüßenswert und durchaus noch ausbaufähig angesehen.

Ausschreibungen werden auch für die Zukunft als wesentliches Element der Universitätsfinanzierung angesehen. Investitionen in Infrastrukturen wie auch Innovationen in der Lehre und die Exzellenz in der Forschung bedürfen weiterer Mittel. Die HRSM bzw. deren „Nachfolgemittel“ werden hierfür als sehr bedeutsam angesehen, nicht zuletzt, weil die kompetitive Mittelausschreibung nicht nur Schwerpunktsetzungen, sondern auch einen Leistungsvergleich ermöglicht. Dementsprechend befürworteten auch alle Interviewpartnerinnen und -partner, die Ergebnisse von HRSM-Projekten noch sichtbarer zu kommunizieren bzw. darzustellen. Die Kommunikation der Projektergebnisse kann/soll dabei durchaus auch in neuen (wie z.B. digitalen) Formaten erfolgen.

Die positive Bewertung der HRSM-Ausschreibungen im Gesamtbild mag auch daran liegen, dass das BMBWF in der Rolle der Programmabwicklung sehr geschätzt wird. Nicht nur, dass die Projektleiterinnen und -leiter den administrativen Aufwand der Antragstellung und die Berichtslegung als angemessen ansehen, darüber hinaus werden auch die stetigen Verbesserungen im Ausschreibungs- und Auswahlverfahren wahrgenommen. So wurde im Vergleich zur HRSM-Ausschreibung 2013 in 2016 die Einbindung externer Expertinnen und Experten im Auswahlverfahren eingeführt. Ergänzend zur Beratung der Kommission war die Einbindung des Qualitätssicherungsrates für Pädagoginnen- und Pädagogenbildung sowie die Einholung weiterer externer Expertise gegeben. Auch haben im Rahmen der Ausschreibung 2016 abgelehnte Projekte Feedback bzw. eine Begründung für die Ablehnung erhalten.

\subsection{Empfehlungen}

Aktuelle Entwicklungen in der Steuerung von Universitäten bzw. Hochschulen - sei es auf europäischer Ebene, oder sei es auf nationaler Ebene - zielen darauf ab, dass Hochschulen zunehmend als Verbünde auftreten und damit mit gemeinsamen Aktivitäten in Lehre und Forschung wie auch im Support eine gesamtsystemische Wirkung erzielen. Als Initiativen können transnational die „European Universities“ oder auch in Deutschland die Bund-Länder-Initiative „Innovative Hochschule“ genannt werden. Beide Initiativen dienen der Hochschulentwicklung - wie auch die Hochschulraum-Strukturmittel - im Verbund bzw. im kooperativen Format. Insbesondere hinsichtlich der Nachhaltigkeit sind der Verbund bzw. die hochschulübergreifende Kooperation von hoher Relevanz, ist doch auszugehen, dass mit Größe mehr Sichtbarkeit und schließlich auch mehr Erfolge, ein 
höherer Impact einhergehen. Vor diesem Hintergrund ist es auch relevant, dass bei künftigen Ausschreibungen folgende Punkte beachtet werden:

1. Verstärktes Augenmerk auf klare Zielsetzungen und damit einhergehend auf den erwartbaren Impact, d.h. dass den Wirkungsdimensionen auf unterschiedlichen Ebenen, wie der Ebene von Kooperationen, der Institutionen- und der Projektebene usw., mehr Beachtung geschenkt wird. Neben einer höheren Verbindlichkeit zur Erreichung von Projektzielen soll damit auch sichergestellt werden, dass sämtliche Potentiale für die Zielerreichung gehoben werden.

2. Eine verbindliche und stärkere Kommunikation der Projektergebnisse nach außen, womit nicht nur die Sichtbarkeit der Projekte, sondern auch die Sichtbarkeit von leistungsfähigen und -starken Akteuren erhöht wird; diese Sichtbarkeit ist ein weiterer Schritt zur Nachhaltigkeit wie auch führt diese - ganz im Sinne eines effizienten Mitteleinsatzes - zu mehr Transparenz im System. Tatsächlich könnte den Hochschulen bereits in der Antragsphase Raum gegeben werden, Vorschläge für die Diffusion ihrer Projektergebnisse zu unterbreiten.

3. HRSM sind als Instrument für die Hochschulentwicklung und nicht als weitere Schiene für die Stärkung von Wissenschaft-Wirtschaftskooperationen zu sehen; wie die HRSM-Ausschreibung 2013 zeigte, stellt das HRSM-Instrument kein attraktives Format für die Beteiligung von Unternehmen an universitären Kooperationsprojekten dar.

4. Hochschulnetzwerken kommt auf nationaler wie auch auf internationaler Ebene eine zunehmend bedeutende Rolle im Hochschul- und Forschungsraum zu. Umso wichtiger ist es, aus Sicht der Governance Acht zu geben, wie sich Netzwerke in der Akteurslandschaft entwickeln. Die Netzwerkanalysen der beiden vorliegenden Evaluierungen zeigen, dass die bislang etablierten Hochschulnetzwerke sehr eigenständig agieren bzw. funktionieren, es gibt kaum Überlappungen. Ob mit dieser Entwicklung sämtliche Potentiale abgeholt werden können, gilt als fraglich. Demnach ist die Entwicklung der Netzwerkbildung im Hochschulraum weiterhin zu beobachten.

Und schließlich ist noch ein finanzieller Aspekt festzuhalten: Ein sensibler Punkt in der Finanzierung betrifft die Definition der Eigenmittel seitens der Universitäten. Einigen Aussagen von Interviewpartnerinnen und -partnern zufolge gibt es diesbezüglich Interpretationsspielräume, eine klare Begriffsdefinition wird für künftige Ausschreibungen als wünschenswert angesehen. 


\section{Literatur}

BMBWF (2018): Universitätsbericht 2017, https://unidata.gv.at/Publikationen/Berichtswesen\%20BMBWF/Universit\%c3\%a4tsbericht/Universit\%c3\%a 4tsbericht\%202014.pdf

BMWF (2013): Kooperationsausschreibung 2013: Anschubfinanzierung von Kooperationen der Universitäten in Lehre und Forschung / Entwicklung und Erschließung der Künste sowie Verwaltung, Wien.

BMWFW (2014): Universitätsbericht 2014, https://unidata.gv.at/Publikationen/Berichtswesen\%20BMBWF/Universit\%c3\%a4tsbericht/Universit\%c3\%a 4tsbericht\%202014.pdf

BMWFW (2016): Hochschulraum-Strukturmittel/Teilbetrag für Kooperationen: Ausschreibung 2016 für den Bereich Lehre, Wien.

Bundesgesetz über die Organisation der Universitäten und ihre Studien: Universitätsgesetz 2002, https://www.ris.bka.gv.at/GeltendeFassung.wxe?Abfrage=Bundesnormen\&Gesetzesnummer=20002128

Dinges, M., Heller-Schuh, B., Kalcik, R., Scherngell, T., Wang, A., Glänzel, W., Thijs, B. (2020): Evaluation FWF Special Research Programmes (SFB), Final Report, https://zenodo.org/record/3889307\#.X5J7gNAzaUk

Europäische Kommission (2014): Measuring the impact of university-business cooperation, Luxemburg https://op.europa.eu/en/publication-detail/-/publication/8c3b24c9-2135-4436-993f-6d09ca87fc5f

Leifeld, P und Malang, T. (2009): Glossar der Politiknetzwerkanalyse (Anhang), VS Verlag für Sozialwissenschaften, Wiesbaden, https://link.springer.com/content/pdf/bbm\%3A978-3-531-918839\%2F1.pdf 


\section{Anhang}

\section{Befragung der Projektleiterinnen und -leiter von finanzierten Projekten der HRSM Ausschreibung 2013}

\section{Anschreiben}

Sehr geehrte Damen und Herren,

im Jahr 2013 hat das Bundesministerium für Bildung, Wissenschaft und Forschung (BMBWF) eine Ausschreibung der Hochschulraumstrukturmittel (im Folgenden kurz: HRSM 2013) zur Anschubfinanzierung von Kooperationen der Universitäten in Lehre und Forschung/Entwicklung und Erschließung der Künste, sowie Verwaltung initiiert bzw. durchgeführt. Insgesamt standen hierfür 63 Mio. € zur Verfügung.

Nachdem nun alle Projekte abgeschlossen sind, hat das BMBWF eine Evaluierung der HRSM-Ausschreibung 2013 veranlasst. Die Evaluierung soll bis Oktober 2020 abgeschlossen werden und wird von WPZ Research und dem AIT Austrian Institute of Technology durchgeführt.

Ziel der Evaluierung ist es, die Qualität der HRSM-Ausschreibung 2013 zu bewerten sowie für künftige Ausschreibungen Anregungen (mitunter Verbesserungsvorschläge) zu erhalten.

Aus diesem Grund ersuchen wir Sie um Teilnahme an der folgenden Umfrage. Die Umfrage wird ca. 15 Minuten Ihrer Zeit in Anspruch nehmen. Die Fragen sind dabei in drei thematische Blöcke gegliedert, nämlich in Fragen zur 1) Antragstellung, 2) Projektumsetzung und 3) Zielerreichung \& Wirkung. Zum Abschluss geben Sie bitte noch eine Grundinformation zu Ihrem Projekt und Ihrer Universität an.

Die Antworten werden automatisch zwischengespeichert. Zwecks Orientierung übermitteln wir Ihnen anbei den Fragebogen auch als PDF. Für die Teilnahme an der Umfrage ersuchen wir Sie aber bitte, unbedingt den OnlineFragebogen auszufüllen. Bitte schließen Sie den Fragebogen bis 15.07.2020 ab.

Alle Antworten werden vertraulich behandelt und anonymisiert auf aggregierter Ebene im Evaluierungsbericht für das BMBWF dargestellt. Die an der Studie beteiligten Expertinnen und Experten unterliegen selbstverständlich der Verschwiegenheitspflicht.

Für Ihre Teilnahme und Unterstützung bedanken wir uns vielmals.

Bei Fragen wenden Sie sich bitte an:

Dr. ${ }^{\text {in }}$ Brigitte Ecker

\section{WPZ}

Research $\mathrm{GmbH}$

Mariahilfer Straße 115/16, 1060 Wien

Tel.: +4318903225

E-Mail: brigitte.ecker@wpz-research.com

Dr. ${ }^{\text {in }}$ Eva Gaisbauer, Mag. Georg Tummeltshammer

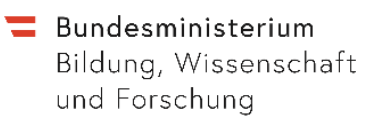

Sektion IV - Universitäten und Fachhochschulen

Abteilung 8 - Finanzierung und Controlling der Universitäten

Minoritenplatz 5, 1010 Wien, Österreich

Tel.: +43 1 53120-5190, + 431 53120-5132

E-Mail: eva.gaisbauer@bmbwf.gv.at, georg.tummeltshammer@bmbwf.gv.at 


\section{Block 1: Zur Antragstellung}

1. Von wem ging die Initiative zur Antragstellung aus?
Von Ihrer Universität
Von einem/einer Kooperationspartner/in

2. Wie lange im Voraus (vor der Einreichung) haben Sie den Antrag vorbereitet?

$\begin{array}{ccccc}\text { weniger als } & 1-3 & 3-6 & \text { länger als } 6 & \text { weiß nicht } \\ 1 \text { Monat } & \text { Monate } & \text { Monate } & \text { Monate } & \end{array}$

3. Aus welchen Gründen haben Sie an der HRSM-Ausschreibung 2013 teilgenommen?

$\square \quad$ Auf- und Ausbau von Lehrinfrastruktur
$\square \quad$ Aufbau neuer gemeinsamer Lehrformate/Studienangebote
$\square \quad$ Weiterentwicklung bestehender Lehrangebote
$\square \quad$ Auf- und Ausbau von Forschungsinfrastruktur
$\square \quad$ Ausbau bestehender Forschungsthemen/Forschungsgruppen
$\square \quad$ Aufbau neuer Forschungsthemen/Forschungsgruppen
$\square \quad$ Nachwuchsförderung
$\square \quad$ Stärkung der Exzellenz
$\square \quad$ Erhöhung der Interdisziplinarität
$\square \quad$ Zugang zu neuen Netzwerken
$\square \quad$ Verbesserung von Verwaltungsstrukturen/-prozessen
$\square \quad$ Aufbau gemeinsamer Servicestrukturen/-leistungen (z.B. im IT-Bereich)
$\square \quad$ Erhöhung der Sichtbarkeit
$\square \quad$ Aufbau nachhaltiger Kooperationsstrukturen
$\square \quad$ Weitere Gründe:

4. Wie beurteilen Sie die Suche nach passenden Kooperationspartnerinnen und -partnern?

sehr schwierig mittel leicht sehrleicht
schwierig

5. Gab es mit Ihren Kooperationspartnerinnen und - partnern bereits eine Zusammenarbeit in anderen Projekten?

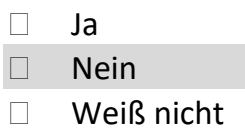

6. Haben Sie im Rahmen Ihres Projekts mit Unternehmen kooperiert?

$\mathrm{Ja}$

Nein 
a. Wenn nein: Aus welchen Gründen nicht?

b. Wenn ja: Aus welchen Gründen haben Sie mit Unternehmen kooperiert?

7. Waren die Ausschreibungsunterlagen in Bezug auf Ziele und Anforderungen klar und nachvollziehbar?

nein ehernein ja $\quad$ ja .

8. Wie beurteilen Sie den administrativen Aufwand für die Antragsstellung der HRSM 2013?

sehrniedrig niedrig hittel hoch sehrhoch

9. Haben Sie die vom BMBWF angebotene Unterstützung im Antragsverfahren in Anspruch genommen?

$\mathrm{Ja}$

Nein

a. Wenn nein: weiter zu Frage 10

b. Wenn ja, wofür haben Sie die Unterstützung durch das BMBWF in Anspruch genommen?
Inhaltliche Fragen
Technische Fragen
Sonstiges:

c. Wenn 9b: Wie beurteilen Sie die vom BMBWF angebotene Unterstützung im Antragsverfahren?

\begin{tabular}{|c|c|}
\hline $\begin{array}{l}\text { gar nicht } \\
\text { hilfreich }\end{array}$ & $\begin{array}{l}\text { eher nicht } \\
\text { hilfreich }\end{array}$ \\
\hline
\end{tabular}

10. Wie beurteilen Sie die Passgenauigkeit/Angemessenheit der Bewertungskriterien (,Indikatorenset und Selbsteinschätzung") der Ausschreibung.

Anm.: Die inhaltlichen Bewertungskriterien für Lehre und Forschung/EEK umfassten: Konzentrationsgrad in der Lehre, Profitmöglichkeiten für Studierende, Etablierung gemeinsamer Strukturen, nachhaltige Kooperation, institutions- bzw. organisationsübergreifende Integration, Strukturbildungsbeitrag bzgl. begonnener inhaltlicher Neuausrichtungen, Management-Knowhow in/von Forschungs-Exzellenzstrukturen, Stärkung existierender Exzellenzbereiche, Nachwuchsförderung und Internationalisierungsgrad.

Anm.: Die inhaltlichen Bewertungskriterien für Verwaltungsprojekte umfassten: nachhaltige Effizienz-steigerung, konkrete Synergieeffekte, Nutzen der Innovation für Studierende bzw. Lehrende, Erhöhung der Transparenz von Verwaltungsabläufen, Teilnahme an universitätsübergreifenden (IT-basierten) Verwaltungsnetzen und Innovationen/Optimierungen im IKT-Bereich.

$$
\text { sehrniedrig niedrig hittel hoch sehrhoch k. A. }
$$


11. Gibt es darunter Bewertungskriterien, die aus Ihrer Sicht schwierig sind bzw. gar nicht passen?

12. Wie schwierig bzw. leicht war es, bei der Antragsstellung einen Bezug zur Leistungsvereinbarung 20132015 bzw. zum Entwicklungsplan der Universität herzustellen?

sehr schwierig mittel leicht sehrleicht k. A.
schwierig

13. Wie bewerten Sie die Nachvollziehbarkeit der Vergabeentscheidung?

sehrniedrig niedrig mittel hoch sehrhoch k. A.

14. Wie bewerten Sie die Angemessenheit der Dauer des Auswahlverfahrens von der Einreichung bis zur Bewilligung?

sehrniedrig niedrig mittel hoch sehr hoch k. A.

15. Wie bewerten Sie die Transparenz des Vergabeverfahrens?

sehrniedrig niedrig hittel hoch sehr hoch

16. Haben Sie noch weitere Anmerkungen oder Verbesserungsvorschläge zum Verfahrensablauf?

\section{Block 2: Zur Projektumsetzung}

17. Wie bewerten Sie die Begrenzung der Finanzierung mit einem Drittel der Projektkosten in der HRSMAusschreibung 2013?

$\begin{array}{lll}\text { zu niedrig } & \text { genau hoch } \\ \text { richtig } & \text { k. A. }\end{array}$

18. Sehen Sie die Beschränkung der HRSM auf ein Drittel der Projektkosten als anreizfördernd oder als behindernd für eine Beteiligung an der HRSM-Ausschreibung?

$$
\text { anreizfördernd behindernd k. A. }
$$


a. Wenn „behindernd“: Was wäre Ihrer Ansicht nach ein anreizfördernder Ansatz zur Finanzierung von Projekten durch HRSM?

19. Durch welche Mittel wurde Ihr HRSM-Projekt noch zusätzlich finanziert?

Globalbudget

Drittmittel

Sonstige/weitere Mittel:

20. War das HRSM-Projekt auch Thema in den Begleitgesprächen zur Leistungsvereinbarung bzw. wurde dieses hier besprochen?

ja nein weiß nicht

21. Wie bewerten Sie die Festlegung des Projekt- und Finanzierungszeitraums mit maximal zwei Leistungsvereinbarungsperioden?

$\begin{array}{ll}\text { zu kurz } & \text { genau } \\ \text { richtig }\end{array}$

a. Wenn „zu kurz“ oder „zu lange“: Was wäre Ihrer Ansicht nach eine adäquate Dauer der Projektlaufzeit bzw. des Finanzierungszeitraums?

\section{Textfeld für Antworten}

22. Wie beurteilen Sie den administrativen Aufwand für das Reporting (d.h. für das Verfassen der Zwischenberichte und Endberichte)?

$$
\begin{array}{ll}
\text { genau niedrig } & \text { zu hoch } \\
\text { richtig }
\end{array}
$$

23. War das Reporting zur Kontrolle des Projektfortschritts und der Zielerreichung zweckmäßig?

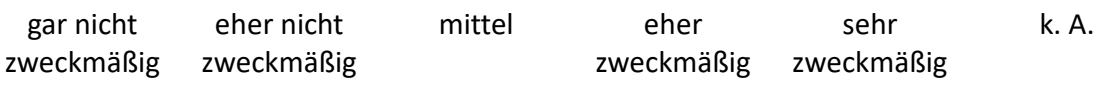

24. Haben Sie Verbesserungsvorschläge zum Reporting? 
25. Wie bewerten Sie die universitätsinterne Unterstützung für Ihr Projekt?

sehrniedrig niedrig hoch sehrhoch k. A.

26. Durch welche Personen hat das HRSM-Projekt universitätsinterne Unterstützung erfahren?

\section{Rektorat}

Fakultätsleitung

Department-/Institutsleitung

Professorinnen/Professoren

Wissenschaftlicher Nachwuchs

Personal in Service- und Administrationsstellen

Sonstige/weitere Unterstützung:

27. Haben Sie noch weitere Anmerkungen oder Verbesserungsvorschläge zur Umsetzung von HRSM-

Projekten?

\section{Block 3: Zur Zielerreichung \& Wirkung}

28. Hat das HRSM-Projekt Ihrer Ansicht nach die grundsätzliche Intention erfüllt?

$\square$ Ja

$\square \quad$ Nein

29. Hinsichtlich der Projektziele - was ist gelungen, was ist nicht so gelungen?

$\begin{array}{llccc}\text { gar nicht } & \text { ehernicht } & \text { eher } & \text { sehr } & \text { nicht } \\ \text { gelungen } & \text { gelungen } & \text { gelungen } & \text { gelungen } & \begin{array}{c}\text { zutreffend/ } \\ \text { weiß nicht }\end{array}\end{array}$

Auf- und Ausbau von Lehrinfrastruktur

\begin{tabular}{|c|c|c|c|c|c|}
\hline $\begin{array}{l}\text { Aufbau neuer gemeinsamer } \\
\text { Lehrformate/Studienangebote }\end{array}$ & $\square$ & $\square$ & $\square$ & $\square$ & $\square$ \\
\hline $\begin{array}{l}\text { Weiterentwicklung } \quad \text { bestehender } \\
\text { Lehrangebote }\end{array}$ & $\square$ & $\square$ & $\square$ & $\square$ & $\square$ \\
\hline $\begin{array}{l}\text { Auf- und Ausbau von } \\
\text { Forschungsinfrastruktur }\end{array}$ & $\square$ & $\square$ & $\square$ & $\square$ & $\square$ \\
\hline $\begin{array}{l}\text { Ausbau bestehender } \\
\text { Forschungsthemen/Forschungsgruppen }\end{array}$ & $\square$ & $\square$ & $\square$ & $\square$ & $\square$ \\
\hline $\begin{array}{l}\text { Aufbau neuer } \\
\text { Forschungsthemen/Forschungsgruppen }\end{array}$ & $\square$ & $\square$ & $\square$ & $\square$ & $\square$ \\
\hline Nachwuchsförderung & $\square$ & $\square$ & $\square$ & $\square$ & $\square$ \\
\hline Stärkung der Exzellenz & $\square$ & $\square$ & $\square$ & $\square$ & $\square$ \\
\hline Erhöhung der Interdisziplinarität & $\square$ & $\square$ & $\square$ & $\square$ & $\square$ \\
\hline Zugang zu neuen Netzwerken & $\square$ & $\square$ & $\square$ & $\square$ & $\square$ \\
\hline
\end{tabular}




\begin{tabular}{|c|c|c|c|c|c|}
\hline & $\begin{array}{l}\text { gar nicht } \\
\text { gelungen }\end{array}$ & $\begin{array}{l}\text { eher nicht } \\
\text { gelungen }\end{array}$ & $\begin{array}{c}\text { eher } \\
\text { gelungen }\end{array}$ & $\begin{array}{l}\text { sehr } \\
\text { gelungen }\end{array}$ & $\begin{array}{c}\text { nicht } \\
\text { zutreffend/ } \\
\text { weiß nicht }\end{array}$ \\
\hline $\begin{array}{ll}\text { Verbesserung } & \text { von } \\
\text { Verwaltungsstrukturen/- prozessen } & \end{array}$ & $\square$ & $\square$ & $\square$ & $\square$ & $\square$ \\
\hline $\begin{array}{l}\text { Aufbau gemeinsamer Servicestrukturen/- } \\
\text { leistungen (z.B. im IT-Bereich) }\end{array}$ & $\square$ & $\square$ & $\square$ & $\square$ & $\square$ \\
\hline Erhöhung der Sichtbarkeit & $\square$ & $\square$ & $\square$ & $\square$ & $\square$ \\
\hline $\begin{array}{ll}\text { Aufbau } & \text { nachhaltiger } \\
\text { Kooperationsstrukturen } & \end{array}$ & $\square$ & $\square$ & $\square$ & $\square$ & $\square$ \\
\hline Sonstiges (genannt bei Frage 2) & $\square$ & $\square$ & $\square$ & $\square$ & $\square$ \\
\hline
\end{tabular}

30. Welchen Zusatznutzen haben die durch die HRSM 2013 finanzierten Projekte der Universität gebracht?

$\begin{aligned} & \square \text { Synergieeffekte } \\ & \square \text { Sichtbarkeit } \\ & \square \text { Größeres Leistungsangebot durch Komplementarität } \\ & \square \quad \text { Kritische Größe } \\ & \square \quad \text { Effizienzgewinne } \\ & \square \text { Sonstige: } \\ & \square\end{aligned}$

31. Inwieweit hat das HRSM-Projekt zur Profilbildung der Universität beigetragen?

\begin{tabular}{|c|c|c|c|}
\hline $\begin{array}{c}\text { gar nicht } \\
\text { beigetragen }\end{array}$ & $\begin{array}{l}\text { eher nicht } \\
\text { beigetragen }\end{array}$ & $\begin{array}{c}\text { eher } \\
\text { beigetragen }\end{array}$ & $\begin{array}{c}\text { sehr } \\
\text { beigetragen }\end{array}$ \\
\hline$\square$ & $\square$ & $\square$ & $\square$ \\
\hline
\end{tabular}

32. Wie ist die Nachhaltigkeit des HRSM-Projekts sichergestellt worden?

$\square$ Durch Verankerung in der Leistungsvereinbarung

$\square$ Durch Veränderung von Strukturen/Prozessen

$\square$ Durch weitere Kooperationsprojekte

$\square$ Durch zusätzliche Finanzierungsmittel

$\square$ Sonstige

33. Wie beurteilen Sie die Nachhaltigkeit der durch die HRSM angestoßenen Kooperationen?

sehrniedrig mittel hoch sehrhoch k. A.


34. Wäre Ihre Universität die Kooperationen auch ohne die HRSM-Finanzierung bzw. das HRSM-Projekt eingegangen?

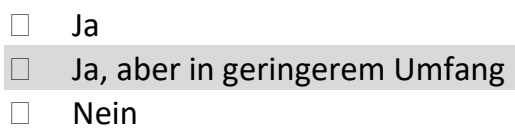

35. Hat die HRSM-Ausschreibung 2013 Ihrer Ansicht nach zu einem besser abgestimmten Hochschul- und Forschungsraum in Österreich beigetragen?

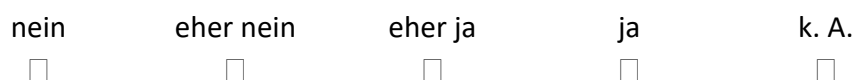

36. Würden Sie sich wieder an einer HRSM-Ausschreibung bzw. einer vergleichbaren Ausschreibung beteiligen?

$\begin{array}{ccccc}\text { nein } & \text { eher nein } & \text { eher ja } & \text { ja } & \text { k. A. } \\ \square & \square & \square & \square & \square\end{array}$

\section{Block 4: Informationen zum Projekt und zur teilnehmenden Universität}

37. Welchen inhaltlichen Schwerpunkt hatte Ihr Projekt?

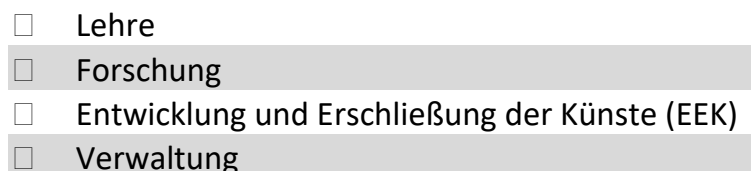

38. Bitte geben Sie Ihre Universität an.

Anm.: Im Rahmen der Auswertung werden die Universitäten zu Universitätsgruppen zusammengefasst, um eine Anonymisierung der Antworten herzustellen.

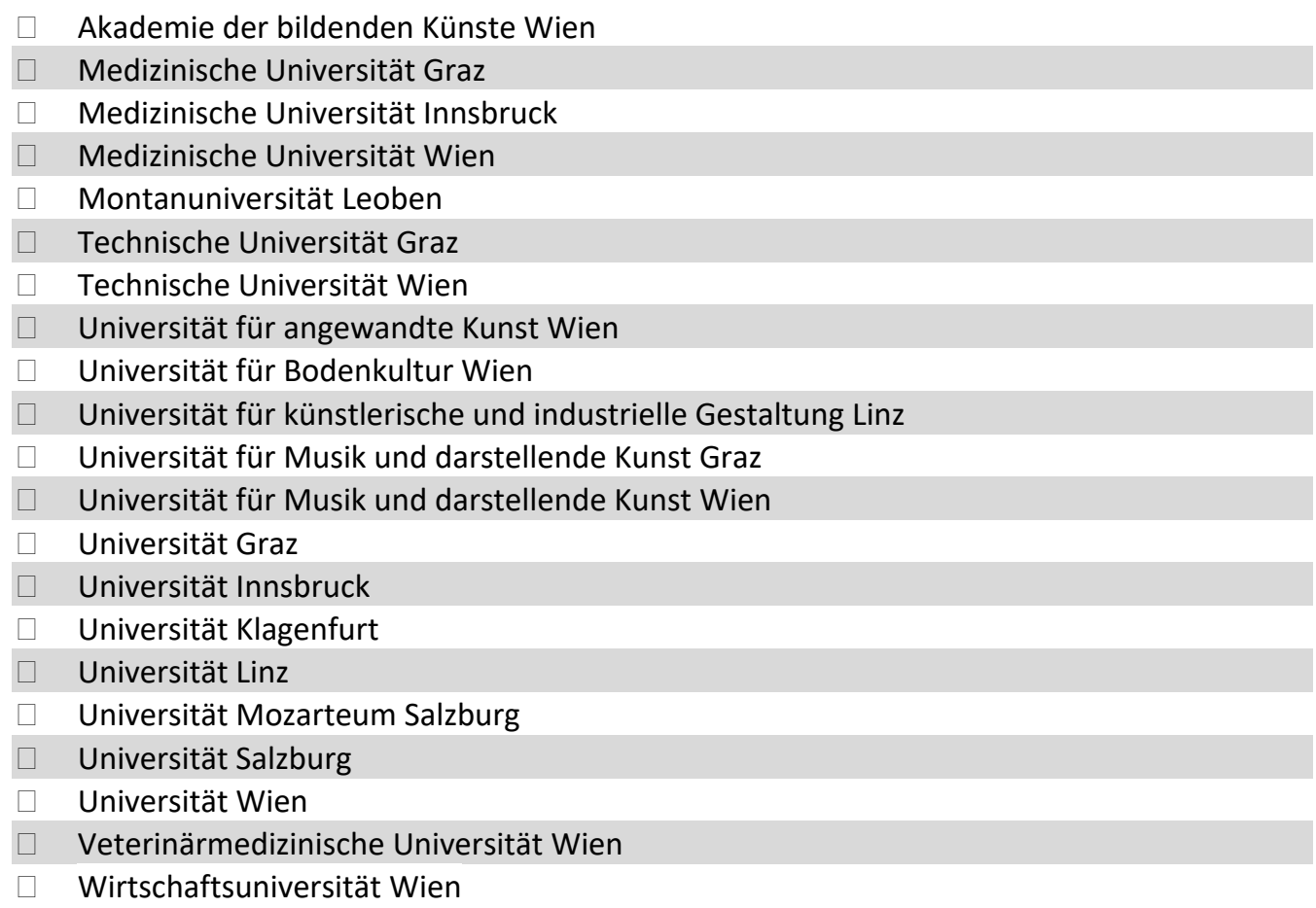


39. Zum Abschluss: Haben Sie neben den angesprochenen Inhalten noch weitere Anmerkungen zur HRSMAusschreibung 2013 oder weiterführende Vorschläge? Wenn ja, bitte geben Sie diese an.

\section{Schluss}

Wir bedanken uns herzlich für Ihr Feedback und Ihre Unterstützung!

Alle Ergebnisse werden ausschließlich für die Kommunikation mit dem BMBWF verwendet. Sollten Sie Fragen haben, stehen wir Ihnen jederzeit gerne zur Verfügung.

Dr. ${ }^{\text {in }}$ Brigitte Ecker

\section{WPZ}

Research GmbH

Mariahilfer Straße 115/16, 1060 Wien

Tel.: +4318903225

E-Mail: brigitte.ecker@wpz-research.com

Dr. ${ }^{\text {in }}$ Eva Gaisbauer, Mag. Georg Tummeltshammer

= Bundesministerium

Bildung, Wissenschaft

und Forschung

Sektion IV - Universitäten und Fachhochschulen

Abteilung 8 - Finanzierung und Controlling der Universitäten

Minoritenplatz 5, 1010 Wien, Österreich

Tel.: +43 1 53120-5190, + 431 53120-5132

E-Mail: eva.gaisbauer@bmbwf.gv.at, georg.tummeltshammer@bmbwf.gv.at 
Befragung der Projektleiterinnen und -leiter von nicht-finanzierten Projekten der HRSMAusschreibung 2013

\section{Anschreiben}

Sehr geehrte Damen und Herren,

im Jahr 2013 hat das Bundesministerium für Bildung, Wissenschaft und Forschung (BMBWF) eine Ausschreibung der Hochschulraumstrukturmittel (im Folgenden kurz: HRSM 2013) zur Anschubfinanzierung von Kooperationen der Universitäten in Lehre und Forschung/Entwicklung und Erschließung der Künste, sowie Verwaltung initiiert bzw. durchgeführt. Insgesamt standen hierfür 63 Mio. € zur Verfügung.

Nachdem nun alle Projekte abgeschlossen sind, hat das BMBWF eine Evaluierung der HRSM-Ausschreibung 2013 veranlasst. Die Evaluierung soll bis Oktober 2020 abgeschlossen werden und wird von WPZ Research und dem AIT Austrian Institute of Technology durchgeführt.

Ziel der Evaluierung ist es, die Qualität der HRSM-Ausschreibung 2013 zu bewerten sowie für künftige Ausschreibungen Anregungen zu erhalten.

Aus diesem Grund ersuchen wir Sie um Teilnahme an der folgenden Umfrage. Auch wenn Ihr Projektantrag nicht genehmigt wurde, wären wir Ihnen dankbar, wenn Sie uns Anregungen, Hinweise und Verbesserungsvorschläge für zukünftige Ausschreibungen seitens des BMBWF geben könnten. Die Umfrage wird ca. 10 Minuten Ihrer Zeit in Anspruch nehmen. Die Fragen sind dabei in drei Blöcke gegliedert, nämlich in Fragen zur 1) Antragstellung des HRSM-Projekts, falls relevant zur 2) Zielerreichung \& Wirkung des mit anderen Mitteln durchgeführten Projekts, sowie zu 3) Hintergrundinformationen Ihren HRSM-Antrag sowie Ihre Universität betreffend.

Die Antworten werden automatisch zwischengespeichert. Zwecks Orientierung übermitteln wir Ihnen anbei den Fragebogen auch als PDF. Für die Teilnahme an der Umfrage ersuchen wir Sie aber bitte, unbedingt den OnlineFragebogen auszufüllen. Bitte schließen Sie den Fragebogen bis 15.07.2020 ab.

Alle Antworten werden vertraulich behandelt und anonymisiert auf aggregierter Ebene im Evaluierungsbericht für das BMBWF dargestellt. Die an der Studie beteiligten Expertinnen und Experten unterliegen selbstverständlich der Verschwiegenheitspflicht.

Für Ihre Teilnahme und Unterstützung bedanken wir uns vielmals.

Bei Fragen wenden Sie sich bitte an:

Dr. ${ }^{\text {in }}$ Brigitte Ecker

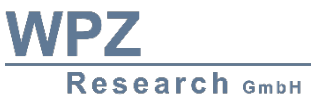

Mariahilfer Straße 115/16, 1060 Wien

Tel.: +4318903225

E-Mail: brigitte.ecker@wpz-research.com

Dr. ${ }^{\text {in }}$ Eva Gaisbauer, Mag. Georg Tummeltshammer

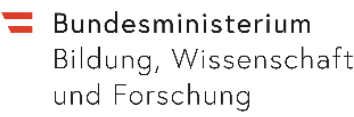

Sektion IV - Universitäten und Fachhochschulen

Abteilung 8 - Finanzierung und Controlling der Universitäten

Minoritenplatz 5, 1010 Wien, Österreich

Tel.: +43 1 53120-5190, + 431 53120-5132

E-Mail: eva.gaisbauer@bmbwf.gv.at, georg.tummeltshammer@bmbwf.gv.at 


\section{Block 1: Zur Antragstellung des HRSM-Projekts}

1. Von wem ging die Initiative zur Antragstellung aus?

Von Ihrer Universität

Von einem/einer Kooperationspartner/in

2. Wie lange im Voraus (vor der Einreichung) haben Sie den Antrag vorbereitet?

$\begin{array}{ccccc}\text { weniger als } & 1-3 & 3-6 & \text { länger als } 6 & \text { weiß nicht } \\ 1 \text { Monat } & \text { Monate } & \text { Monate } & \text { Monate } & \end{array}$

3. Aus welchen Gründen wollten Sie an der HRSM-Ausschreibung 2013 teilnehmen?

\begin{tabular}{ll}
$\square \quad$ Auf- und Ausbau von Forschungsinfrastruktur \\
$\square \quad$ Ausbau bestehender Forschungsthemen/Forschungsgruppen \\
$\square \quad$ Aufbau neuer Forschungsthemen/Forschungsgruppen \\
$\square \quad$ Nachwuchsförderung \\
$\square \quad$ Stärkung der Exzellenz \\
$\square \quad$ Erhöhung der Interdisziplinarität \\
$\square \quad$ Zugang zu neuen Netzwerken \\
$\square \quad$ Aufbau und Weiterentwicklung gemeinsamer Studienangebote \\
$\square \quad$ Verbesserung von Verwaltungsstrukturen/-prozessen \\
$\square \quad$ Aufbau gemeinsamer Servicestrukturen/-leistungen (z.B. im IT-Bereich) \\
$\square \quad$ Erhöhung der Sichtbarkeit \\
$\square \quad$ Aufbau nachhaltiger Kooperationsstrukturen \\
$\square \quad$ Weitere Gründe: \\
\hline
\end{tabular}

4. Wie beurteilen Sie die Suche nach passenden Kooperationspartnerinnen und -partnern?

sehr schwierig mittel leicht sehrleicht k. A.
schwierig

5. Gab es mit Ihren Kooperationspartnerinnen und - partnern bereits eine Zusammenarbeit in anderen Projekten?

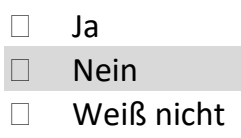

6. Wollten Sie im Rahmen Ihres Projekts mit Unternehmen kooperiert?
Ja
Nein 
a. Wenn nein: Aus welchen Gründen nicht?

b. Wenn ja: Aus welchen Gründen wollten Sie mit Unternehmen kooperiert?

7. Waren die Ausschreibungsunterlagen in Bezug auf Ziele und Anforderungen klar und nachvollziehbar?

nein ehernein ja $\quad$ ja .

8. Wie beurteilen Sie den administrativen Aufwand für die Antragsstellung der HRSM 2013?

sehrniedrig niedrig hittel hoch sehrhoch

9. Haben Sie die vom BMBWF angebotene Unterstützung im Antragsverfahren in Anspruch genommen?

$\mathrm{Ja}$

Nein

a. Wenn nein: weiter zu Frage 10

b. Wenn ja, wofür haben Sie die Unterstützung durch das BMBWF in Anspruch genommen?
Inhaltliche Fragen
Technische Fragen
Sonstiges:

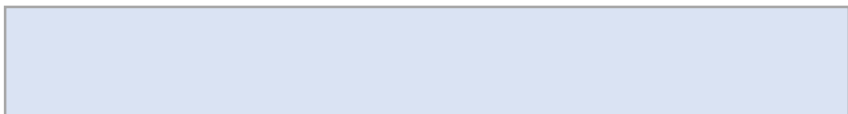

c. Wenn 6b: Wie beurteilen Sie die vom BMBWF angebotene Unterstützung im Antragsverfahren?

$\begin{array}{lccc}\text { gar nicht } & \text { eher nicht } & \text { eher } & \text { sehr } \\ \text { hilfreich } & \text { hilfreich } & \text { hilfreich } & \text { hilfreich }\end{array}$

10. Wie beurteilen Sie die Passgenauigkeit/Angemessenheit der Bewertungskriterien („Indikatorenset und Selbsteinschätzung") der Ausschreibung.

Anm.: Die inhaltlichen Bewertungskriterien für Lehre und Forschung/EEK umfassten: Konzentrationsgrad in der Lehre, Profitmöglichkeiten für Studierende, Etablierung gemeinsamer Strukturen, nachhaltige Kooperation, institutions- bzw. organisationsübergreifende Integration, Strukturbildungsbeitrag bzgl. begonnener inhaltlicher Neuausrichtungen, Management-Knowhow in/von Forschungs-Exzellenzstrukturen, Stärkung existierender Exzellenzbereiche, Nachwuchsförderung und Internationalisierungsgrad.

Anm.: Die inhaltlichen Bewertungskriterien für Verwaltungsprojekte umfassten: nachhaltige Effizienz-steigerung, konkrete Synergieeffekte, Nutzen der Innovation für Studierende bzw. Lehrende, Erhöhung der Transparenz von Verwaltungsabläufen, Teilnahme an universitätsübergreifenden (IT-basierten) Verwaltungs-netzen und Innovationen/Optimierungen im IKT-Bereich.

sehrniedrig niedrig hoch sehrhoch k. A. 
11. Gibt es darunter Bewertungskriterien, die aus Ihrer Sicht schwierig sind bzw. gar nicht passen?

12. Wie bewerten Sie die Begrenzung der Finanzierung mit einem Drittel der Projektkosten in der HRSMAusschreibung 2013?

$\begin{array}{lll}\text { zu niedrig } & \text { genau hoch } \\ \text { richtig }\end{array}$

13. Sehen Sie die Beschränkung der HRSM auf ein Drittel der Projektkosten als anreizfördernd oder als behindernd für eine Beteiligung an der HRSM-Ausschreibung?

$$
\text { anreizfördernd behindernd k.A. }
$$

a. Wenn „behindernd“: Was wäre Ihrer Ansicht nach ein anreizfördernder Ansatz zur Finanzierung von Projekten durch HRSM?

14. Wie bewerten Sie die Nachvollziehbarkeit der Vergabeentscheidung?

sehrniedrig niedrig hittel hoch sehrhoch A.

15. Wie bewerten Sie die Angemessenheit der Dauer des Auswahlverfahrens von der Einreichung bis zur Entscheidung?

$\begin{array}{cccccc}\text { sehr niedrig } & \text { niedrig } & \text { mittel } & \text { hoch } & \text { sehr hoch } & \text { k. A. } \\ \square & \square & \square & \square & \square & \square\end{array}$

16. Wie bewerten Sie die Transparenz des Vergabeverfahrens?

sehrniedrig niedrig moch sehrhoch k. A.

17. Ist es für Sie nachvollziehbar, warum bzw. aufgrund welcher Kriterien ihr Projektantrag abgelehnt wurde?

nein ehernein ja ja

18. Haben Sie Anmerkungen, Verbesserungsvorschläge oder Hinweise, wie das Antragsverfahren in Zukunft noch besser gestaltet werden kann? 


\section{Block 2: Zur Zielerreichung \& Wirkung des mit anderen Mitteln durchgeführten Projekts}

19. Ist Ihr Kooperationsprojekt auch ohne die HRSM-Finanzierung zustande gekommen?

$\mathrm{Ja}$

Ja, aber in geringerem Umfang

Nein

Wenn Ja: Fragen 20-22 und dann weiter zu Frage 24

Wenn Nein: weiter zu Frage 23

20. Wenn ja, durch welche Mittel?

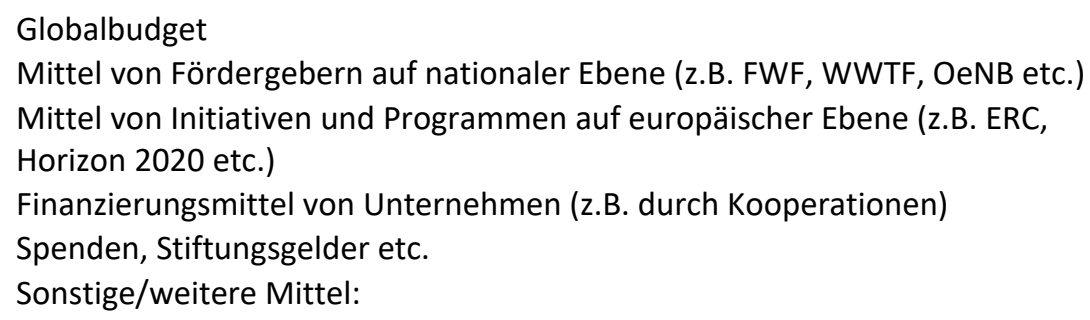

21. Hinsichtlich der Projektziele - was ist gelungen, was ist nicht so gelungen?

$$
\begin{array}{llccc}
\text { gar nicht } & \text { eher nicht } & \text { eher } & \text { sehr } & \text { nicht } \\
\text { gelungen } & \text { gelungen } & \text { gelungen } & \text { gelungen } & \text { zutreffend/ }
\end{array}
$$

\begin{tabular}{|c|c|c|c|c|c|}
\hline $\begin{array}{l}\text { Aufbau neuer gemeinsamer } \\
\text { Lehrformate/Studienangebote }\end{array}$ & $\square$ & $\square$ & $\square$ & $\square$ & $\square$ \\
\hline $\begin{array}{l}\text { Weiterentwicklung } \quad \text { bestehender } \\
\text { Lehrangebote }\end{array}$ & $\square$ & $\square$ & $\square$ & $\square$ & $\square$ \\
\hline $\begin{array}{l}\text { Auf- und Ausbau von } \\
\text { Forschungsinfrastruktur }\end{array}$ & $\square$ & $\square$ & $\square$ & $\square$ & $\square$ \\
\hline $\begin{array}{l}\text { Ausbau bestehender } \\
\text { Forschungsthemen/Forschungsgruppen }\end{array}$ & $\square$ & $\square$ & $\square$ & $\square$ & $\square$ \\
\hline $\begin{array}{l}\text { Aufbau neuer } \\
\text { Forschungsthemen/Forschungsgruppen }\end{array}$ & $\square$ & $\square$ & $\square$ & $\square$ & $\square$ \\
\hline Nachwuchsförderung & $\square$ & $\square$ & $\square$ & $\square$ & $\square$ \\
\hline Stärkung der Exzellenz & $\square$ & $\square$ & $\square$ & $\square$ & $\square$ \\
\hline Erhöhung der Interdisziplinarität & $\square$ & $\square$ & $\square$ & $\square$ & $\square$ \\
\hline Zugang zu neuen Netzwerken & $\square$ & $\square$ & $\square$ & $\square$ & $\square$ \\
\hline $\begin{array}{ll}\text { Verbesserung } & \text { von } \\
\text { Verwaltungsstrukturen/- prozessen } & \end{array}$ & $\square$ & $\square$ & $\square$ & $\square$ & $\square$ \\
\hline $\begin{array}{l}\text { Aufbau gemeinsamer Servicestrukturen/- } \\
\text { leistungen (z.B. im IT-Bereich) }\end{array}$ & $\square$ & $\square$ & $\square$ & $\square$ & $\square$ \\
\hline
\end{tabular}

Auf- und Ausbau von Lehrinfrastruktur 


\begin{tabular}{|c|c|c|c|c|c|c|}
\hline & & $\begin{array}{l}\text { gar nicht } \\
\text { gelungen }\end{array}$ & $\begin{array}{l}\text { eher nicht } \\
\text { gelungen }\end{array}$ & $\begin{array}{c}\text { eher } \\
\text { gelungen }\end{array}$ & $\begin{array}{c}\text { sehr } \\
\text { gelungen }\end{array}$ & $\begin{array}{c}\text { nicht } \\
\text { zutreffend/ } \\
\text { weiß nicht }\end{array}$ \\
\hline $\begin{array}{l}\text { Aufbau } \\
\text { Kooperationsstrukturen }\end{array}$ & nachhaltiger & $\square$ & $\square$ & $\square$ & $\square$ & $\square$ \\
\hline Sonstiges (genannt bei Fr & & $\square$ & $\square$ & $\square$ & $\square$ & $\square$ \\
\hline
\end{tabular}

22. Welchen Zusatznutzen hat Ihr Projekt der Universität gebracht?

\begin{aligned} &$\square$ Synergieeffekte \\ &$\square$ Sichtbarkeit \\ &$\square$ Größeres Leistungsangebot durch Komplementarität \\ &$\square \quad$ Kritische Größe \\ &$\square \quad$ Effizienzgewinne \\ &$\square$ Sonstige: \\ &$\quad \\ &$\hline\end{aligned}

23. Inwieweit hat Ihr Projekt zur Profilbildung der Universität beigetragen?

\begin{tabular}{|c|c|c|c|}
\hline $\begin{array}{l}\text { gar nicht } \\
\text { beigetragen }\end{array}$ & $\begin{array}{l}\text { eher nicht } \\
\text { beigetragen }\end{array}$ & $\begin{array}{c}\text { eher } \\
\text { beigetragen } \\
\square\end{array}$ & $\begin{array}{c}\text { sehr } \\
\text { beigetragen } \\
\square\end{array}$ \\
\hline
\end{tabular}

24. Ist die Nachhaltigkeit des Projekts sichergestellt worden?

$$
\begin{aligned}
& \text { Ja } \\
& \text { Teilweise } \\
& \text { Nein }
\end{aligned}
$$

Wenn Ja und Teilweise: Frage 25

Wenn Nein: weiter zu Frage 26

25. Wie ist die Nachhaltigkeit Ihres Projekts sichergestellt worden?

$$
\begin{aligned}
& \text { Durch Verankerung in der Leistungsvereinbarung } \\
& \text { Durch Veränderung von Strukturen/Prozessen } \\
& \text { Durch weitere Kooperationsprojekte } \\
& \text { Durch zusätzliche Finanzierungsmittel } \\
& \text { Sonstige }
\end{aligned}
$$

26. Welche Voraussetzungen oder Rahmenbedingungen wären notwendig gewesen, um Ihr Kooperationsprojekt auch ohne Hochschulraumstrukturmittel umzusetzen? 
27. Haben sich bereits bzw. werden Sie sich wieder an einer HRSM-Ausschreibung bzw. einer vergleichbaren Ausschreibung beteiligen?

nein ehernein ja $\quad$ ja $\quad$ k.

\section{Block 3: Informationen zum HRSM-Projekt und zur teilnehmenden Universität}

28. Welchen inhaltlichen Schwerpunkt hatte Ihr beantragtes HRSM-Projekt?

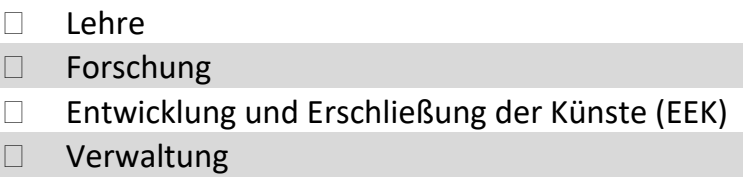

29. Bitte geben Sie Ihre Universität an.

Anm.: Im Rahmen der Auswertung werden die Universitäten zu Universitätsgruppen zusammengefasst, um eine Anonymisierung der Antworten herzustellen.

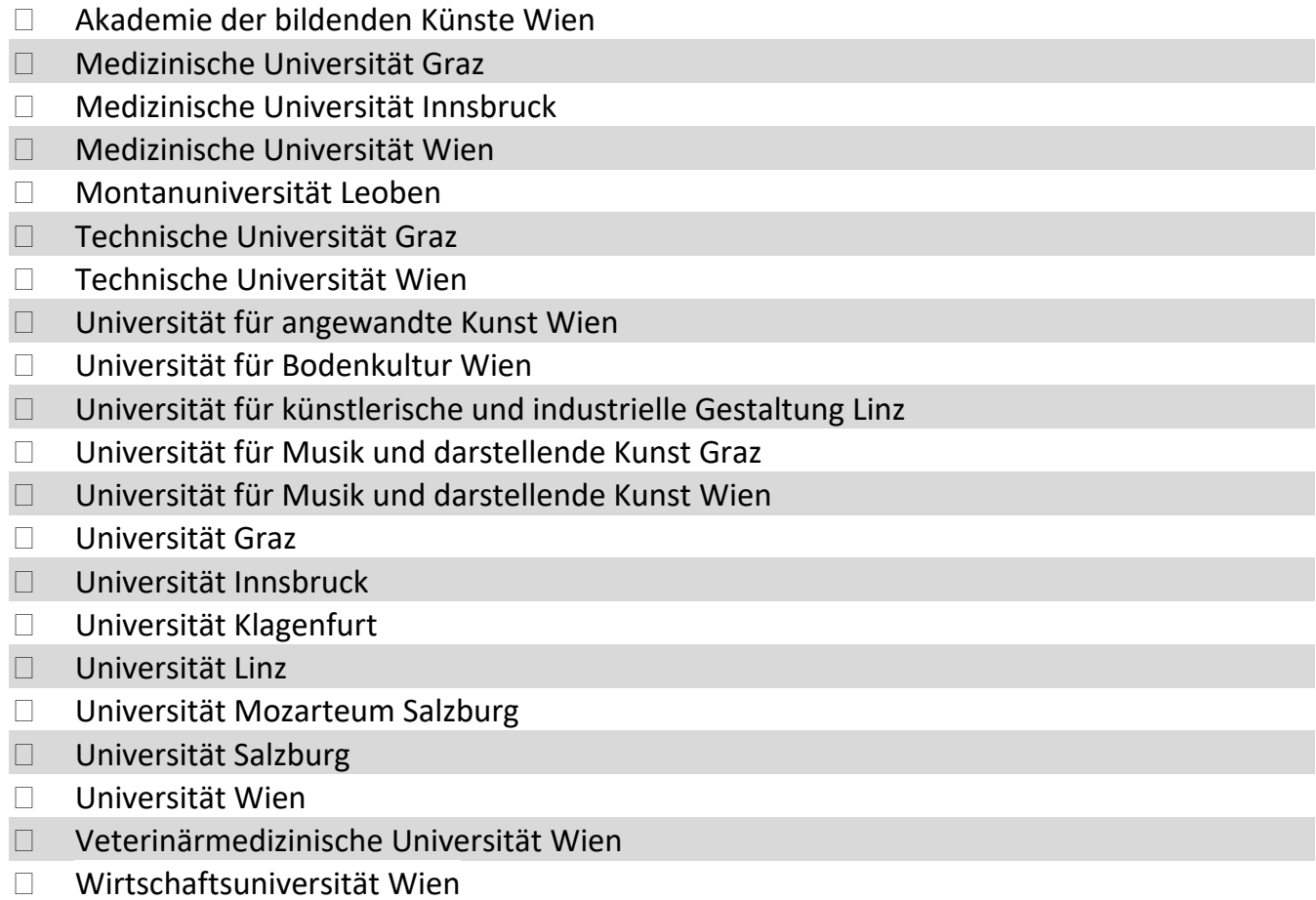

30. Zum Abschluss: Haben Sie neben den angesprochenen Inhalten noch weitere Anmerkungen zur HRSMAusschreibung 2013 oder weiterführende Vorschläge? Wenn ja, bitte geben Sie diese infolge an. 


\section{Schluss}

Wir bedanken uns herzlich für Ihr Feedback und Ihre Unterstützung!

Alle Ergebnisse werden ausschließlich für die Kommunikation mit dem BMBWF verwendet. Sollten Sie Fragen haben, stehen wir Ihnen jederzeit gerne zur Verfügung.

Dr. ${ }^{\text {in }}$ Brigitte Ecker

\section{WPZ}

Research GmbH

Mariahilfer Straße 115/16, 1060 Wien

Tel.: +4318903225

E-Mail: brigitte.ecker@wpz-research.com

Dr. ${ }^{\text {in }}$ Eva Gaisbauer, Mag. Georg Tummeltshammer

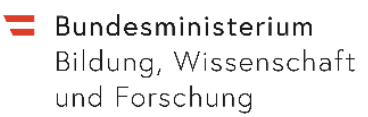

Sektion IV - Universitäten und Fachhochschulen

Abteilung 8 - Finanzierung und Controlling der Universitäten

Minoritenplatz 5, 1010 Wien, Österreich

Tel.: +43 1 53120-5190, + 431 53120-5132

E-Mail: eva.gaisbauer@bmbwf.gv.at, georg.tummeltshammer@bmbwf.gv.at 
Befragung der Projektleiterinnen und -leiter von finanzierten Projekten der HRSMAusschreibung für Kooperationen im Bereich Lehre 2016

\section{Anschreiben}

Sehr geehrte Damen und Herren,

im Jahr 2016 hat das Bundesministerium für Bildung, Wissenschaft und Forschung (BMBWF) eine Ausschreibung der Hochschulraumstrukturmittel (im Folgenden kurz: HRSM 2016) zur Anschubfinanzierung von Kooperationen der Universitäten in der Lehre mit dem Schwerpunkt auf der Pädagoginnen- und Pädagogenbildung NEU durchgeführt. Im Speziellen gab es dabei auch eine Anschubfinanzierung von exzellenzfördernden und strukturentwickelnden Kooperationsprojekten im Bereich Lehre. Insgesamt standen hierfür Mittel in Höhe von 35 Mio. € zur Verfügung.

Nachdem nun alle Kooperationsprojekte im Bereich Lehre umgesetzt bzw. abgeschlossen sind, hat das BMBWF eine Evaluierung der HRSM-Ausschreibung 2016 bei den teilnehmenden Universitäten, Hochschulen und Forschungseinrichtungen veranlasst. Die Evaluierung soll bis Oktober 2020 abgeschlossen werden und wird von WPZ Research und dem AIT Austrian Institute of Technology durchgeführt.

Ziel der Evaluierung ist es, die Qualität der HRSM-Ausschreibung 2016 für strukturbildende, qualitätsverbessernde Maßnahmen für in Kooperation eingerichtete Bachelor- und Masterstudien im Bereich des Lehramts und für exzellenzfördernde und strukturentwickelnde Kooperationsprojekte im Bereich Lehre zu bewerten, sowie für künftige Ausschreibungen Anregungen und Verbesserungsvorschläge zu erhalten.

Aus diesem Grund ersuchen wir Sie um Teilnahme an der folgenden Umfrage. Die Umfrage wird ca. 15 Minuten Ihrer Zeit in Anspruch nehmen. Die Fragen sind dabei in drei thematische Blöcke gegliedert, nämlich in Fragen zur 1) Antragstellung, 2) Projektumsetzung und 3) Zielerreichung \& Wirkung. Zum Abschluss geben Sie bitte noch eine Grundinformation zu Ihrem Projekt und Ihrer Universität an.

Die Antworten werden automatisch zwischengespeichert. Zwecks Orientierung übermitteln wir Ihnen anbei den Fragebogen auch als PDF. Für die Teilnahme an der Umfrage ersuchen wir Sie, aber bitte unbedingt den OnlineFragebogen auszufüllen. Bitte schließen Sie den Fragebogen bis 15.07.2020 ab.

Alle Antworten werden vertraulich behandelt und anonymisiert auf aggregierter Ebene im Evaluierungsbericht für das BMBWF dargestellt. Die an der Studie beteiligten Expertinnen und Experten unterliegen selbstverständlich der Verschwiegenheitspflicht.

Für Ihre Teilnahme und Unterstützung bedanken wir uns vielmals.

Dr. ${ }^{\text {in }}$ Brigitte Ecker

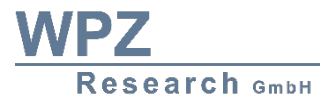

Mariahilfer Straße 115/16, 1060 Wien

Tel.: +4318903225

E-Mail: brigitte.ecker@wpz-research.com

Dr. ${ }^{\text {in }}$ Eva Gaisbauer, Mag. Georg Tummeltshammer

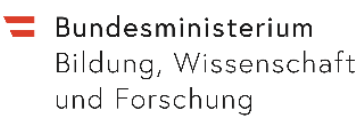

Sektion IV - Universitäten und Fachhochschulen

Abteilung 8 - Finanzierung und Controlling der Universitäten

Minoritenplatz 5, 1010 Wien, Österreich

Tel.: +43153120-5190, + 431 53120-5132

E-Mail: eva.gaisbauer@bmbwf.gv.at, georg.tummeltshammer@bmbwf.gv.at 


\section{Block 1: Zur Antragstellung}

1. Von wem ging die Initiative zur Antragstellung aus?

Von Ihrer Universität

Von einem/einer Kooperationspartner/in

2. Wie lange im Voraus (vor der Einreichung) haben Sie den Antrag vorbereitet?

$\begin{array}{ccccc}\text { weniger als } & 1-3 & 3-6 & \text { länger als } 6 & \text { weiß nicht } \\ 1 \text { Monat } & \text { Monate } & \text { Monate } & \text { Monate } & \end{array}$

3. Aus welchen Gründen haben Sie an der HRSM-Ausschreibung 2016, im Speziellen für exzellenzfördernde und strukturentwickelnde Kooperationsprojekte im Bereich Lehre, teilgenommen?

\begin{tabular}{l}
$\square \quad$ Aufbau neuer universitätsübergreifender Studienangebote (inkl. Lehramtsstudien) \\
$\square \quad$ Weiterentwicklung von universitätsübergreifenden Studienangeboten (inkl. Lehramtsstudien) \\
$\square \quad$ Ausbau von MINT-Fächern \\
$\square \quad$ Unterstützung des Personalaufbaus und der Personalentwicklung in der Lehre \\
$\square \quad$ Stärkung der Verbundregionen zur Durchführung von Lehramtsstudien \\
$\square \quad$ Innovation und fachliche Neuerungen \\
$\square \quad$ Gewährleistung und Verbesserung der Qualität der Lehre in Lehramtsstudien \\
$\square \quad$ Nachwuchsförderung \\
$\square \quad$ Erhöhung der Diversität/Inklusion von nicht-traditionellen Studierenden \\
$\square \quad$ Verringerung des Dropouts \\
$\square \quad$ Förderung von Frauen \\
$\square \quad$ Auf- und Ausbau von Infrastrukturen zwecks Nutzung der Digitalisierung in der Lehre \\
$\square \quad$ Einsatz von MOOCs (massive open online courses) und anderen E-Learning Formaten \\
$\square \quad$ Verbesserung der innerösterreichischen Mobilität von Studierenden \\
$\square \quad$ Aufbau nachhaltiger Kooperationsstrukturen \\
\hline$\quad$ Weitere Gründe: \\
\begin{tabular}{|l}
\hline \\
$\square \quad$
\end{tabular} \\
\hline
\end{tabular}

4. Wie beurteilen Sie die Suche nach passenden Kooperationspartnerinnen und -partnern?

sehr schwierig mittel leicht sehrleicht
schwierig

5. Gab es mit Ihren Kooperationspartnerinnen und -partnern bereits eine Zusammenarbeit in anderen Projekten?
Ja
Nein
Weiß nicht

6. Waren die Ausschreibungsunterlagen in Bezug auf Ziele und Anforderungen klar und nachvollziehbar?

nein eher nein eherja ja $\quad$ k. A.


7. Wie beurteilen Sie den administrativen Aufwand für die Antragsstellung der HRSM 2016?

sehrniedrig niedrig hoch sehrhoch k. A.

8. Haben Sie die vom BMBWF angebotene Unterstützung im Antragsverfahren in Anspruch genommen?

$\mathrm{Ja}$

Nein

a. Wenn nein: weiter zu Frage 9

b. Wenn ja, wofür haben Sie die Unterstützung durch das BMBWF in Anspruch genommen?

Inhaltliche Fragen

Technische Fragen

Sonstiges:

c. Wenn 9b: Wie beurteilen Sie die vom BMBWF angebotene Unterstützung im Antragsverfahren?

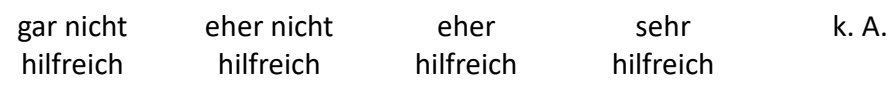

9. Wie beurteilen Sie die Passgenauigkeit/Angemessenheit der Bewertungskriterien (,Indikatorenset und Selbsteinschätzung") der Ausschreibung.

Anm.: Die Bewertungskriterien für Projekte der PädagogInnenbildung NEU umfassten: Etablierung gemeinsamer Strukturen im Bereich des Lehrens und Lernens für nachhaltige Kooperationen auch über die konkrete Projektdauer hinaus; Sicherung und Weiterentwicklung der wissenschaftlichen und professionsorientierten Voraussetzungen durch die Schaffung kooperativer, insbesondere auch universitätsübergreifender Arbeitseinheiten in den Verbundregionen zur Durchführung der Lehramtsstudien unter besonderer Berücksichtigung des zu erwartenden Bedarfs an Absolventinnen und Absolventen; Einrichtung und Weiterentwicklung von Lehramtsstudien unter Nutzung sämtlicher vorhandener Ressourcen innerhalb einer Verbundregion und ggf. über diese hinausgehend unter besonderer Berücksichtigung des zu erwartenden Bedarfs an Absolventinnen und Absolventen; Gewährleistung einer qualitätsvollen Lehre in besonders frequentierten Lehramtsstudien unter Berücksichtigung des Bedarfs an Absolventinnen und Absolventen; Förderung des wissenschaftlichen Nachwuchses, insbesondere für die Fachdidaktiken und den Primarbereich (z.B. Doktorandenkollegs, (Verbund-)Professuren, o.ä.); Innovation und fachliche Neuerungen, wie z.B. fächerübergreifende Angebote zur Förderung von MINT-Fächern etc.

Anm.: Die Bewertungskriterien für sonstige Lehrprojekte umfassten: Etablierung gemeinsamer Strukturen im Bereich des Lehrens und Lernens für nachhaltige Kooperationen auch über die konkrete Projektdauer hinaus; Entwicklung universitätsübergreifender Studienangebote unter Nutzung vorhandener Ressourcen und Synergien; konkrete Maßnahmen zur Erhöhung der Studienaktivität und der Abschlussquoten; institutions- bzw. organisationsübergreifende Integration und Betreuung nicht-traditioneller Studierender (z.B. berufsbegleitendes Studium, Förderung von Studierenden aus bildungsfernen Schichten, Förderung der gleichen Teilhabe von Männern und Frauen an einzelnen Studienfächern (insb. MINT-Fächer); Innovationen/Optimierungen im Bereich von IT-basierten Lehr- und Lernformaten; sowie Erleichterung der innerösterreichischen Mobilität von Studierenden.

sehrniedrig niedrig mittel hoch sehr hoch 
10. Gibt es darunter Bewertungskriterien, die aus Ihrer Sicht schwierig sind bzw. gar nicht passen?

11. Wie schwierig bzw. leicht war es, bei der Antragsstellung einen Bezug zur Leistungsvereinbarung 20162018 bzw. zum Entwicklungsplan der Universität herzustellen?

sehr schwierig mittel leicht sehrleicht
schwierig

12. Wie bewerten Sie die Nachvollziehbarkeit der Vergabeentscheidung?

sehrniedrig niedrig hoch sehrhoch k. A.

13. Wie bewerten Sie die Angemessenheit der Dauer des Auswahlverfahrens von der Einreichung bis zur Bewilligung?

sehrniedrig niedrig hittel hoch sehr hoch $\quad$ k. A.

14. Wie bewerten Sie die Transparenz des Vergabeverfahrens?

sehrniedrig niedrig hoch sehrhoch k. A.

15. Haben Sie noch weitere Anmerkungen oder Verbesserungsvorschläge zum Verfahrensablauf?

\section{Block 2: Zur Projektumsetzung}

16. Wie bewerten Sie die Höhe der HRSM-Finanzierung im Verhältnis zu den Gesamtkosten des Projekts?

$\begin{array}{lll}\text { zu niedrig } & \text { genau } \\ \text { richtig } & \text { ku hoch A. }\end{array}$

17. In der Regel wurde bis zu einem Drittel der Projektkosten als Anschubfinanzierung vom BMBWF übernommen. In sachlich begründeten Fällen und nach einstimmiger Empfehlung der Auswahlkommission wurden auch mehr als ein Drittel der Kosten vom BMBWF übernommen. Sehen Sie diese Regelung als anreizfördernd oder als behindernd für eine Beteiligung an der HRSM-Ausschreibung an?

$$
\text { anreizfördernd behindernd k. A. }
$$


a. Wenn „,behindernd“: Was wäre Ihrer Ansicht nach ein anreizfördernder Ansatz zur Finanzierung von Projekten durch HRSM?

18. Durch welche Mittel wurde Ihr HRSM-Projekt noch zusätzlich finanziert?
Globalbudget
Drittmittel
Sonstige/weitere Mittel:

19. War das HRSM-Projekt auch Thema in den Begleitgesprächen zur Leistungsvereinbarung bzw. wurde dieses hier besprochen?

$$
\text { ja nein weiß nicht }
$$

20. Wie bewerten Sie die Dauer des Projekt- und Finanzierungszeitraums?

$$
\begin{array}{ll}
\text { zu kurz } & \begin{array}{l}
\text { genau } \\
\text { richtig }
\end{array}
\end{array}
$$

a. Wenn „zu kurz“ oder „zu lange“: Was wäre Ihrer Ansicht nach eine adäquate Dauer der Projektlaufzeit bzw. des Finanzierungszeitraums?

21. Wie beurteilen Sie den administrativen Aufwand für das Reporting (d.h. für das Verfassen der Zwischenberichte und Endberichte)?

$$
\begin{array}{ll}
\text { zenau niedrig } & \text { zu hoch } \\
\text { richtig }
\end{array}
$$

22. War das Reporting zur Kontrolle des Projektfortschritts und der Zielerreichung zweckmäßig?

$\begin{array}{ccccc}\text { gar nicht } & \text { eher nicht } & \text { mittel } & \text { eher } & \text { sehr } \\ \text { zweckmäßig } & \text { zweckmäßig } & & \text { zweckmäßig } & \text { zweckmäßig }\end{array}$

23. Haben Sie Verbesserungsvorschläge zum Reporting? 
24. Wie bewerten Sie die universitätsinterne Unterstützung für Ihr Projekt?

sehrniedrig niedrig hoch sehrhoch k. A.

25. Durch welche Personen hat das HRSM-Projekt universitätsinterne Unterstützung erfahren?

\section{Rektorat}

Fakultätsleitung

Department-/Institutsleitung

Professorinnen/Professoren

Wissenschaftlicher Nachwuchs

Personal in Service- und Administrationsstellen

Sonstige/weitere Unterstützung:

26. Haben Sie noch weitere Anmerkungen oder Verbesserungsvorschläge zur Umsetzung von HRSMProjekten?

\section{Block 3: Zur Zielerreichung \& Wirkung}

27. Hat das HRSM-Projekt Ihrer Ansicht nach die grundsätzliche Intention erfüllt?

Ja

Nein

28. Hinsichtlich der Projektziele - was ist gelungen, was ist nicht so gelungen?

$\begin{array}{ccccc}\text { gar nicht } & \text { ehernicht } & \text { eher } & \text { sehr } & \text { nicht } \\ \text { gelungen } & \text { gelungen } & \text { gelungen } & \text { gelungen } & \begin{array}{c}\text { zutreffend/ } \\ \text { weiß nicht }\end{array}\end{array}$

Aufbau neuer universitätsübergreifender Studienangebote (inkl. Lehramtsstudien)

\begin{tabular}{|c|c|c|c|c|c|}
\hline $\begin{array}{l}\text { Weiterentwicklung von } \\
\text { universitätsübergreifenden } \\
\text { Studienangeboten (inkl. Lehramtsstudien) }\end{array}$ & $\square$ & $\square$ & $\square$ & $\square$ & $\square$ \\
\hline Ausbau von MINT-Fächern & $\square$ & $\square$ & $\square$ & $\square$ & $\square$ \\
\hline $\begin{array}{l}\text { Unterstützung des Personalaufbaus und } \\
\text { der Personalentwicklung in der Lehre }\end{array}$ & $\square$ & $\square$ & $\square$ & $\square$ & $\square$ \\
\hline $\begin{array}{l}\text { Stärkung der Verbundregionen zur } \\
\text { Durchführung von Lehramtsstudien }\end{array}$ & $\square$ & $\square$ & $\square$ & $\square$ & $\square$ \\
\hline Innovation und fachliche Neuerungen & $\square$ & $\square$ & $\square$ & $\square$ & $\square$ \\
\hline
\end{tabular}




$\begin{array}{ccccc}\text { gar nicht } & \text { eher nicht } & \text { eher } & \text { sehr } & \text { nicht } \\ \text { gelungen } & \text { gelungen } & \text { gelungen } & \text { gelungen } & \begin{array}{c}\text { zutreffend/ } \\ \text { weiß nicht }\end{array}\end{array}$

Gewährleistung und Verbesserung der Qualität der Lehre in Lehramtsstudien

\begin{tabular}{|c|c|c|c|c|c|}
\hline Nachwuchsförderung & $\square$ & $\square$ & $\square$ & $\square$ & $\square$ \\
\hline $\begin{array}{l}\text { Erhöhung der Diversität/Inklusion von } \\
\text { nicht-traditionellen Studierenden }\end{array}$ & $\square$ & $\square$ & $\square$ & $\square$ & $\square$ \\
\hline Verringerung des Dropouts & $\square$ & $\square$ & $\square$ & $\square$ & $\square$ \\
\hline Förderung von Frauen & $\square$ & $\square$ & $\square$ & $\square$ & $\square$ \\
\hline $\begin{array}{l}\text { Auf- und Ausbau von Infrastrukturen } \\
\text { zwecks Nutzung der Digitalisierung in der } \\
\text { Lehre }\end{array}$ & $\square$ & $\square$ & $\square$ & $\square$ & $\square$ \\
\hline $\begin{array}{l}\text { Einsatz von MOOCs (massive open online } \\
\text { courses) und anderen E-Learning } \\
\text { Formaten }\end{array}$ & $\square$ & $\square$ & $\square$ & $\square$ & $\square$ \\
\hline $\begin{array}{l}\text { Verbesserung der innerösterreichischen } \\
\text { Mobilität von Studierenden }\end{array}$ & $\square$ & $\square$ & $\square$ & $\square$ & $\square$ \\
\hline $\begin{array}{ll}\text { Aufbau } & \text { nachhaltiger } \\
\text { Kooperationsstrukturen } & \end{array}$ & $\square$ & $\square$ & $\square$ & $\square$ & $\square$ \\
\hline Sonstige Ziele (genannt bei Frage 3) & $\square$ & $\square$ & $\square$ & $\square$ & $\square$ \\
\hline
\end{tabular}

29. Welchen Zusatznutzen haben die durch die HRSM 2016 finanzierten Projekte der Universität gebracht?

$\square$ Synergieeffekte

Sichtbarkeit

Größeres Leistungsangebot durch Komplementarität

Kritische Größe

Effizienzgewinne

Sonstige:

30. Wie ist die Nachhaltigkeit des HRSM-Projekts sichergestellt worden?

$\square$ Durch Verankerung in der Leistungsvereinbarung

Durch Veränderung von Strukturen/Prozessen

Durch weitere Kooperationsprojekte

$\square$ Durch zusätzliche Finanzierungsmittel

$\square$ Sonstige: 
31. Wie beurteilen Sie die Nachhaltigkeit der durch die HRSM angestoßenen Kooperationen?

sehrniedrig niedrig hoch sehrhoch k. A.

32. Sind innovative Ansätze aus dem HRSM-Projekt in den Regelbetrieb der Universität übernommen worden?

nein ja weiß nicht

a. Wenn ja, welche innovativen Ansätze wurden in den Regelbetrieb der Universität übernommen?

33. Wäre Ihre Universität die Kooperationen im Bereich Lehre auch ohne die HRSM-Finanzierung bzw. das HRSM-Projekt eingegangen?

$\square \mathrm{Ja}$

Ja, aber in geringerem Umfang

Nein

34. Hat die HRSM-Ausschreibung 2016 Ihrer Ansicht nach zu einem besser abgestimmten österreichischen Hochschulraum beigetragen?

nein ehernein eher ja ja $\quad$ k.

35. Würden Sie sich wieder an einer HRSM-Ausschreibung bzw. einer vergleichbaren Ausschreibung beteiligen?

nein ehernein eherja ja $\quad$ k. A.

\section{Block 4: Informationen zum Projekt und zur teilnehmenden Universität}

36. Welchen Schwerpunkt hatte Ihr Projekt?

Pädagoginnen- und Pädagogenbildung NEU

Sonstiges Lehrprojekt

37. Bitte geben Sie Ihre Universität an.

Anm.: Im Rahmen der Auswertung werden die Universitäten zu Universitätsgruppen zusammengefasst, um eine Anonymisierung der Antworten herzustellen.

Akademie der bildenden Künste Wien

Medizinische Universität Graz

Medizinische Universität Innsbruck

Medizinische Universität Wien

Montanuniversität Leoben

Technische Universität Graz

Technische Universität Wien 


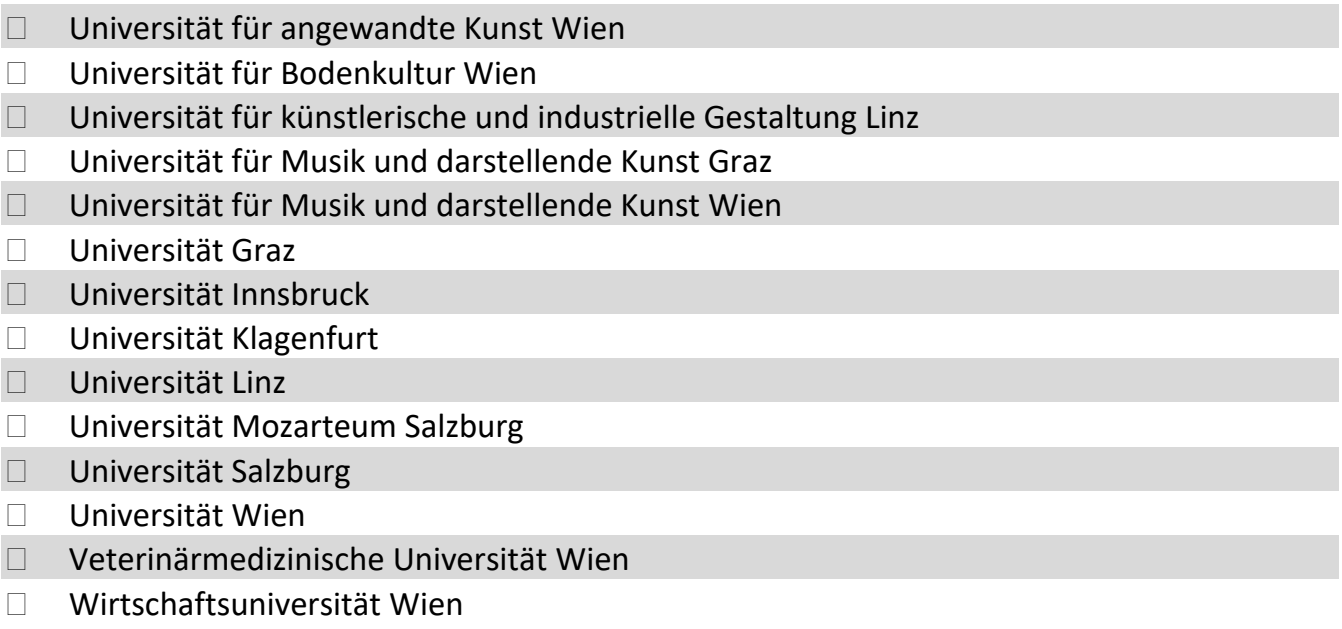

38. Zum Abschluss: Haben Sie neben den angesprochenen Inhalten noch weitere Anmerkungen zur HRSMAusschreibung 2016 oder weiterführende Vorschläge? Wenn ja, bitte geben Sie diese infolge an.

\section{Schluss}

Wir bedanken uns herzlich für Ihr Feedback und Ihre Unterstützung!

Alle Ergebnisse werden ausschließlich für die Kommunikation mit dem BMBWF verwendet. Sollten Sie Fragen haben, stehen wir Ihnen jederzeit gerne zur Verfügung.

Dr. ${ }^{\text {in }}$ Brigitte Ecker

\section{WPZ}

Mariahilfer Straße 115/16, 1060 Wien

Tel.: +4318903225

E-Mail: brigitte.ecker@wpz-research.com

Dr. ${ }^{\text {in }}$ Eva Gaisbauer, Mag. Georg Tummeltshammer

$$
\begin{aligned}
= & \text { Bundesministerium } \\
& \text { Bildung, Wissenschaft } \\
& \text { und Forschung }
\end{aligned}
$$

Sektion IV - Universitäten und Fachhochschulen

Abteilung 8 - Finanzierung und Controlling der Universitäten

Minoritenplatz 5, 1010 Wien, Österreich

Tel.: +43 1 53120-5190, + 431 53120-5132

E-Mail: eva.gaisbauer@bmbwf.gv.at, georg.tummeltshammer@bmbwf.gv.at 
Befragung der Projektleiterinnen und -leiter von nicht-finanzierten Projekten der HRSMAusschreibung 2016

Anschreiben

Sehr geehrte Damen und Herren,

im Jahr 2016 hat das Bundesministerium für Bildung, Wissenschaft und Forschung (BMBWF) eine Ausschreibung der Hochschulraumstrukturmittel (im Folgenden kurz: HRSM 2016) zur Anschubfinanzierung von Kooperationen der Universitäten in der Lehre mit dem Schwerpunkt auf der Pädagoginnen- und Pädagogenbildung NEU durchgeführt. Im Speziellen gab es dabei auch eine Anschubfinanzierung von exzellenzfördernden und strukturentwickelnden Kooperationsprojekten im Bereich Lehre. Insgesamt standen hierfür Mittel in Höhe von 35 Mio. € zur Verfügung.

Nachdem nun alle Kooperationsprojekte im Bereich Lehre umgesetzt bzw. abgeschlossen sind, hat das BMBWF eine Evaluierung der HRSM-Ausschreibung 2016 bei den teilnehmenden Universitäten, Hochschulen und Forschungseinrichtungen veranlasst. Die Evaluierung soll bis Oktober 2020 abgeschlossen werden und wird von WPZ Research und dem AIT Austrian Institute of Technology durchgeführt.

Ziel der Evaluierung ist es, die Qualität der HRSM-Ausschreibung 2016 für strukturbildende, qualitätsverbessernde Maßnahmen für in Kooperation eingerichtete Bachelor- und Masterstudien im Bereich des Lehramts und für exzellenzfördernde und strukturentwickelnde Kooperationsprojekte im Bereich Lehre zu bewerten, sowie für künftige Ausschreibungen Anregungen und Verbesserungsvorschläge zu erhalten.

Aus diesem Grund ersuchen wir Sie um Teilnahme an der folgenden Umfrage. Auch wenn Ihr Projektantrag nicht genehmigt wurde, wären wir Ihnen dankbar, wenn Sie uns Anregungen, Hinweise und Verbesserungsvorschläge für zukünftige Ausschreibungen seitens des BMBWF geben könnten. Die Umfrage wird ca. 10 Minuten Ihrer Zeit in Anspruch nehmen. Die Fragen sind dabei in drei Blöcke gegliedert, nämlich in Fragen zur 1) Antragstellung des HRSM-Projekts, falls relevant zur 2) Zielerreichung \& Wirkung des mit anderen Mitteln durchgeführten Projekts, sowie zu 3) Hintergrundinformationen Ihren HRSM-Antrag sowie Ihre Universität betreffend.

Die Antworten werden automatisch zwischengespeichert. Zwecks Orientierung übermitteln wir Ihnen anbei den Fragebogen auch als PDF. Für die Teilnahme an der Umfrage ersuchen wir Sie aber bitte, unbedingt den OnlineFragebogen auszufüllen. Bitte schließen Sie den Fragebogen bis 15.07.2020 ab.

Alle Antworten werden vertraulich behandelt und anonymisiert auf aggregierter Ebene im Evaluierungsbericht für das BMBWF dargestellt. Die an der Studie beteiligten Expertinnen und Experten unterliegen selbstverständlich der Verschwiegenheitspflicht.

Für Ihre Teilnahme und Unterstützung bedanken wir uns vielmals. Bei Fragen wenden Sie sich bitte an:

Dr. ${ }^{\text {in }}$ Brigitte Ecker

WPZ

Research $\mathrm{GmbH}$

Mariahilfer Straße 115/16, 1060 Wien

Tel.: +4318903225

E-Mail: brigitte.ecker@wpz-research.com

Dr. ${ }^{\text {in }}$ Eva Gaisbauer, Mag. Georg Tummeltshammer

- Bundesministerium

Bildung, Wissenschaft

und Forschung

Sektion IV - Universitäten und Fachhochschulen

Abteilung 8 - Finanzierung und Controlling der Universitäten

Minoritenplatz 5, 1010 Wien, Österreich

Tel.: +43 1 53120-5190, + 431 53120-5132

E-Mail: eva.gaisbauer@bmbwf.gv.at, georg.tummeltshammer@bmbwf.gv.at 


\section{Block 1: Zur Antragstellung des HRSM-Projekts}

1. Von wem ging die Initiative zur Antragstellung aus?

Von Ihrer Universität

Von einem/einer Kooperationspartner/in

2. Wie lange im Voraus (vor der Einreichung) haben Sie den Antrag vorbereitet?

$\begin{array}{ccccc}\text { weniger als } & 1-3 & 3-6 & \text { länger als } 6 & \text { weiß nicht } \\ 1 \text { Monat } & \text { Monate } & \text { Monate } & \text { Monate } & \end{array}$

3. Aus welchen Gründen haben Sie an der HRSM-Ausschreibung 2016, im Speziellen für exzellenzfördernde und strukturentwickelnde Kooperationsprojekte im Bereich Lehre, teilgenommen?

\begin{tabular}{|c|c|}
\hline 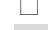 & Autbau neuer universitatsubergreitend \\
\hline$\square$ & Weiterentwicklung von universitätsübergreifenden Studienangeboten (inkl. Lehramtsstudien) \\
\hline$\square$ & Ausbau von MINT-Fächern \\
\hline$\square$ & Unterstützung des Personalaufbaus und der Personalentwicklung in der Lehre \\
\hline$\square$ & Stärkung der Verbundregionen zur Durchführung von Lehramtsstudien \\
\hline$\square$ & Innovation und fachliche Neuerungen \\
\hline$\square$ & Gewährleistung und Verbesserung der Qualität der Lehre in Lehramtsstudien \\
\hline$\square$ & Nachwuchsförderung \\
\hline$\square$ & Erhöhung der Diversität/Inklusion von nicht-traditionellen Studierenden \\
\hline$\square$ & Verringerung des Dropouts \\
\hline$\square$ & Förderung von Frauen \\
\hline$\square$ & Auf- und Ausbau von Infrastrukturen zwecks Nutzung der Digitalisierung in der Lehre \\
\hline$\square$ & Einsatz von MOOCs (massive open online courses) und anderen E-Learning Formaten \\
\hline$\square$ & Verbesserung der innerösterreichischen Mobilität von Studierenden \\
\hline$\square$ & Aufbau nachhaltiger Kooperationsstrukturen \\
\hline$\square$ & Weitere Gründe: \\
\hline
\end{tabular}

4. Wie beurteilen Sie die Suche nach passenden Kooperationspartnerinnen und -partnern?

sehr schwierig mittel leicht sehrleicht
schwierig

5. Gab es mit Ihren Kooperationspartnerinnen und - partnern bereits eine Zusammenarbeit in anderen Projekten?

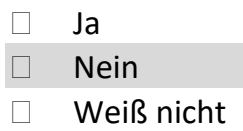

6. Waren die Ausschreibungsunterlagen in Bezug auf Ziele und Anforderungen klar und nachvollziehbar?

nein eher nein $\quad$ eher ja $\quad$ ja $\quad$ k. A.


7. Wie beurteilen Sie den administrativen Aufwand für die Antragsstellung der HRSM 2013?

sehrniedrig niedrig hoch sehrhoch k. A.

8. Haben Sie die vom BMBWF angebotene Unterstützung im Antragsverfahren in Anspruch genommen?

Ja

Nein

a. Wenn nein: weiter zu Frage 9

b. Wenn ja, wofür haben Sie die Unterstützung durch das BMBWF in Anspruch genommen?

Inhaltliche Fragen

Technische Fragen

Sonstiges:

c. Wenn 6b: Wie beurteilen Sie die vom BMBWF angebotene Unterstützung im Antragsverfahren?

$\begin{array}{lccc}\text { gar nicht } & \text { eher nicht } & \text { eher } & \text { sehr } \\ \text { hilfreich } & \text { hilfreich } & \text { hilfreich } & \text { hilfreich }\end{array}$

9. Wie beurteilen Sie die Passgenauigkeit/Angemessenheit der Bewertungskriterien („,Indikatorenset und Selbsteinschätzung") der Ausschreibung.

Anm.: Die Bewertungskriterien für Projekte der PädagogInnenbildung NEU umfassten: Etablierung gemeinsamer Strukturen im Bereich des Lehrens und Lernens für nachhaltige Kooperationen auch über die konkrete Projektdauer hinaus; Sicherung und Weiterentwicklung der wissenschaftlichen und professionsorientierten Voraussetzungen durch die Schaffung kooperativer, insbesondere auch universitätsübergreifender Arbeitseinheiten in den Verbundregionen zur Durchführung der Lehramtsstudien unter besonderer Berücksichtigung des zu erwartenden Bedarfs an Absolventinnen und Absolventen; Einrichtung und Weiterentwicklung von Lehramtsstudien unter Nutzung sämtlicher vorhandener Ressourcen innerhalb einer Verbundregion und ggf. über diese hinausgehend unter besonderer Berücksichtigung des zu erwartenden Bedarfs an Absolventinnen und Absolventen; Gewährleistung einer qualitätsvollen Lehre in besonders frequentierten Lehramtsstudien unter Berücksichtigung des Bedarfs an Absolventinnen und Absolventen; Förderung des wissenschaftlichen Nachwuchses, insbesondere für die Fachdidaktiken und den Primarbereich (z.B. Doktorandenkollegs, (Verbund-)Professuren, o.ä.); Innovation und fachliche Neuerungen, wie z.B. fächerübergreifende Angebote zur Förderung von MINT-Fächern etc.

Anm.: Die Bewertungskriterien für sonstige Lehrprojekte umfassten: Etablierung gemeinsamer Strukturen im Bereich des Lehrens und Lernens für nachhaltige Kooperationen auch über die konkrete Projektdauer hinaus; Entwicklung universitätsübergreifender Studienangebote unter Nutzung vorhandener Ressourcen und Synergien; konkrete Maßnahmen zur Erhöhung der Studienaktivität und der Abschlussquoten; institutions- bzw. organisationsübergreifende Integration und Betreuung nicht-traditioneller Studierender (z.B. berufsbegleitendes Studium, Förderung von Studierenden aus bildungsfernen Schichten, Förderung der gleichen Teilhabe von Männern und Frauen an einzelnen Studienfächern (insb. MINT-Fächer); Innovationen/Optimierungen im Bereich von IT-basierten Lehr- und Lernformaten; sowie Erleichterung der innerösterreichischen Mobilität von Studierenden.

sehrniedrig niedrig mittel hoch sehr hoch

10. Gibt es darunter Bewertungskriterien, die aus Ihrer Sicht schwierig sind bzw. gar nicht passen? 
11. In der Regel wurde bis zu einem Drittel der Projektkosten als Anschubfinanzierung vom BMBWF übernommen. In sachlich begründeten Fällen und nach einstimmiger Empfehlung der Auswahlkommission wurden auch mehr als ein Drittel der Kosten vom BMBWF übernommen. Sehen Sie diese Regelung als anreizfördernd oder als behindernd für eine Beteiligung an der HRSM-Ausschreibung an? anreizfördernd behindernd k. A.

a. Wenn „behindernd“: Was wäre Ihrer Ansicht nach ein anreizfördernder Ansatz zur Finanzierung von Projekten durch HRSM?

12. Wie bewerten Sie die Nachvollziehbarkeit der Vergabeentscheidung?

sehrniedrig niedrig hoch sehrhoch k. A.

13. Wie bewerten Sie die Angemessenheit der Dauer des Auswahlverfahrens von der Einreichung bis zur Entscheidung?

sehrniedrig niedrig hoch sehrhoch k. A.

14. Wie bewerten Sie die Transparenz des Vergabeverfahrens?

sehrniedrig niedrig hoch sehrhoch k. A.

15. Ist es für Sie nachvollziehbar, warum bzw. aufgrund welcher Kriterien ihr Projektantrag abgelehnt wurde?

nein ehernein ja ja

16. Haben Sie Anmerkungen, Verbesserungsvorschläge oder Hinweise, wie das Antragsverfahren in Zukunft noch besser gestaltet werden kann?

\section{Block 2: Zur Zielerreichung \& Wirkung des mit anderen Mitteln durchgeführten Projekts}

17. Ist Ihr Kooperationsprojekt auch ohne die HRSM-Finanzierung zustande gekommen?

$\mathrm{Ja}$

Ja, aber in geringerem Umfang

Nein

Wenn Ja: Fragen 18-23 und dann Frage 25

Wenn Nein: weiter zu Frage 24 
18. Wenn ja, durch welche Mittel?

$\square$ Globalbudget

$\square$ Mittel von Fördergebern auf nationaler Ebene (z.B. FWF, WWTF, OeNB etc.)

$\square$ Mittel von Initiativen und Programmen auf europäischer Ebene (z.B. ERC, Horizon 2020 etc.)

Finanzierungsmittel von Unternehmen (z.B. durch Kooperationen)

Spenden, Stiftungsgelder etc.

Sonstige/weitere Mittel:

19. Hinsichtlich der Projektziele - was ist gelungen, was ist nicht so gelungen?

$\begin{array}{llccc}\text { gar nicht } & \text { eher nicht } & \text { eher } & \text { sehr } & \text { nicht } \\ \text { gelungen } & \text { gelungen } & \text { gelungen } & \text { gelungen } & \begin{array}{c}\text { zutreffend/ } \\ \text { weiß nicht }\end{array}\end{array}$

Aufbau neuer universitätsübergreifender

Studienangebote (inkl. Lehramtsstudien)

\begin{tabular}{|c|c|c|c|c|c|}
\hline $\begin{array}{l}\text { Weiterentwicklung von } \\
\text { universitätsübergreifenden } \\
\text { Studienangeboten (inkl. Lehramtsstudien) }\end{array}$ & $\square$ & $\square$ & $\square$ & $\square$ & $\square$ \\
\hline Ausbau von MINT-Fächern & $\square$ & $\square$ & $\square$ & $\square$ & $\square$ \\
\hline $\begin{array}{l}\text { Unterstützung des Personalaufbaus und } \\
\text { der Personalentwicklung in der Lehre }\end{array}$ & $\square$ & $\square$ & $\square$ & $\square$ & $\square$ \\
\hline
\end{tabular}

Stärkung der Verbundregionen zur Durchführung von Lehramtsstudien

Innovation und fachliche Neuerungen

$\square \quad \square$

$\square$

Gewährleistung und Verbesserung der Qualität der Lehre in Lehramtsstudien

\begin{tabular}{|c|c|c|c|c|c|}
\hline Nachwuchsförderung & $\square$ & $\square$ & $\square$ & $\square$ & $\square$ \\
\hline $\begin{array}{l}\text { Erhöhung der Diversität/Inklusion von } \\
\text { nicht-traditionellen Studierenden }\end{array}$ & $\square$ & $\square$ & $\square$ & $\square$ & $\square$ \\
\hline Verringerung des Dropouts & $\square$ & $\square$ & $\square$ & $\square$ & $\square$ \\
\hline Förderung von Frauen & $\square$ & $\square$ & $\square$ & $\square$ & $\square$ \\
\hline $\begin{array}{l}\text { Auf- und Ausbau von Infrastrukturen } \\
\text { zwecks Nutzung der Digitalisierung in der } \\
\text { Lehre }\end{array}$ & $\square$ & $\square$ & $\square$ & $\square$ & $\square$ \\
\hline $\begin{array}{l}\text { Einsatz von MOOCs (massive open online } \\
\text { courses) und anderen E-Learning } \\
\text { Formaten }\end{array}$ & $\square$ & $\square$ & $\square$ & $\square$ & $\square$ \\
\hline $\begin{array}{l}\text { Verbesserung der innerösterreichischen } \\
\text { Mobilität von Studierenden }\end{array}$ & $\square$ & $\square$ & $\square$ & $\square$ & $\square$ \\
\hline $\begin{array}{ll}\text { Aufbau } & \text { nachhaltiger } \\
\text { Kooperationsstrukturen } & \end{array}$ & $\square$ & $\square$ & $\square$ & $\square$ & $\square$ \\
\hline Sonstige Ziele (genannt bei Frage 3) & $\square$ & $\square$ & $\square$ & $\square$ & $\square$ \\
\hline
\end{tabular}


20. Welchen Zusatznutzen hat Ihr Projekt der Universität gebracht?

Synergieeffekte

Sichtbarkeit

] Größeres Leistungsangebot durch Komplementarität

Kritische Größe

$\square$ Effizienzgewinne

Sonstige:

21. Inwieweit hat Ihr Projekt zur Profilbildung der Universität beigetragen?

\begin{tabular}{|c|c|c|c|}
\hline $\begin{array}{l}\text { gar nicht } \\
\text { beigetragen }\end{array}$ & $\begin{array}{l}\text { eher nicht } \\
\text { beigetragen }\end{array}$ & $\begin{array}{c}\text { eher } \\
\text { beigetragen }\end{array}$ & $\begin{array}{c}\text { sehr } \\
\text { beigetragen }\end{array}$ \\
\hline
\end{tabular}

22. Ist die Nachhaltigkeit des Projekts sichergestellt worden?

Ja

Teilweise

Nein

Wenn Ja und Teilweise: Frage 23

Wenn Nein: weiter zu Frage 25

23. Wie ist die Nachhaltigkeit Ihres Projekts sichergestellt worden?

$\square$ Durch Verankerung in der Leistungsvereinbarung

Durch Veränderung von Strukturen/Prozessen

$\square$ Durch weitere Kooperationsprojekte

Durch zusätzliche Finanzierungsmittel

Sonstige

24. Welche Voraussetzungen oder Rahmenbedingungen wären notwendig gewesen, um Ihr Kooperationsprojekt auch ohne Hochschulraumstrukturmittel umzusetzen?

25. Haben Sie sich bereits bzw. werden Sie sich wieder an einer HRSM-Ausschreibung bzw. einer vergleichbaren Ausschreibung beteiligen?

nein ehernein eher ja k. A.




\section{Block 3: Informationen zum HRSM-Projekt und zur teilnehmenden Universität}

26. Welchen inhaltlichen Schwerpunkt hatte Ihr beantragtes HRSM-Projekt?

Pädagoginnen- und Pädagogenbildung NEU

Sonstiges Lehrprojekt

27. Bitte geben Sie Ihre Universität an.

Anm.: Im Rahmen der Auswertung werden die Universitäten zu Universitätsgruppen zusammengefasst, um eine Anonymisierung der Antworten herzustellen.

\begin{tabular}{ll}
$\square \quad$ Akademie der bildenden Künste Wien \\
$\square \quad$ Medizinische Universität Graz \\
$\square \quad$ Medizinische Universität Innsbruck \\
$\square \quad$ Medizinische Universität Wien \\
$\square \quad$ Montanuniversität Leoben \\
$\square \quad$ Technische Universität Graz \\
$\square \quad$ Technische Universität Wien \\
$\square \quad$ Universität für angewandte Kunst Wien \\
$\square \quad$ Universität für Bodenkultur Wien \\
$\square \quad$ Universität für künstlerische und industrielle Gestaltung Linz \\
$\square \quad$ Universität für Musik und darstellende Kunst Graz \\
$\square \quad$ Universität für Musik und darstellende Kunst Wien \\
$\square \quad$ Universität Graz \\
$\square \quad$ Universität Innsbruck \\
$\square \quad$ Universität Klagenfurt \\
$\square \quad$ Universität Linz \\
\hline$\quad$ Universität Mozarteum Salzburg \\
$\square \quad$ Universität Salzburg \\
$\square \quad$ Universität Wien \\
$\square \quad$ Veterinärmedizinische Universität Wien \\
$\square \quad$ Wirtschaftsuniversität Wien
\end{tabular}

28. Zum Abschluss: Haben Sie neben den angesprochenen Inhalten noch weitere Anmerkungen zur HRSMAusschreibung 2016 oder weiterführende Vorschläge? Wenn ja, bitte geben Sie diese infolge an. 


\section{Schluss}

Wir bedanken uns herzlich für Ihr Feedback und Ihre Unterstützung!

Alle Ergebnisse werden ausschließlich für die Kommunikation mit dem BMBWF verwendet. Sollten Sie Fragen haben, stehen wir Ihnen jederzeit gerne zur Verfügung.

Dr. ${ }^{\text {in }}$ Brigitte Ecker

\section{WPZ}

Mariahilfer Straße 115/16, 1060 Wien

Tel.: +4318903225

E-Mail: brigitte.ecker@wpz-research.com

Dr. ${ }^{\text {in }}$ Eva Gaisbauer, Mag. Georg Tummeltshammer

$$
\begin{aligned}
= & \text { Bundesministerium } \\
& \text { Bildung, Wissenschaft } \\
& \text { und Forschung }
\end{aligned}
$$

Sektion IV - Universitäten und Fachhochschulen

Abteilung 8 - Finanzierung und Controlling der Universitäten

Minoritenplatz 5, 1010 Wien, Österreich

Tel.: +43 1 53120-5190, + 431 53120-5132

E-Mail: eva.gaisbauer@bmbwf.gv.at, georg.tummeltshammer@bmbwf.gv.at 
Autorinnen und Autoren: Brigitte Ecker, Clemens Danler, Gerald Gogola, Nikolas Reschen, Anna Wang

Titel: Evaluierung der HRSM-Ausschreibung 2013 zur Anschubfinanzierung von Kooperationen der Universitäten in Lehre und Forschung/Entwicklung und Erschließung der Künste sowie Verwaltung und Evaluierung der HRSM-Ausschreibung 2016 für Kooperationen im Bereich Lehre

Projektbericht

(C) 2021 WPZ Research GmbH

Mariahilfer Straße 115/16, A-1060 Wien • Tel. +43 $118903225 \bullet \underline{\text { http://www.wpz-research.com }}$ 